Cochrane Database of Systematic Reviews

\title{
Interventions for improving modifiable risk factor control in the secondary prevention of stroke (Review)
}

Bridgwood B, Lager KE, Mistri AK, Khunti K, Wilson AD, Modi P

Bridgwood B, Lager KE, Mistri AK, Khunti K, Wilson AD, Modi P.

Interventions for improving modifiable risk factor control in the secondary prevention of stroke.

Cochrane Database of Systematic Reviews 2018, Issue 5. Art. No.: CD009103.

DOI: 10.1002/14651858.CD009103.pub3.

www.cochranelibrary.com 
TABLE OF CONTENTS

ABSTRACT

PLAIN LANGUAGE SUMMARY

SUMMARY OF FINDINGS

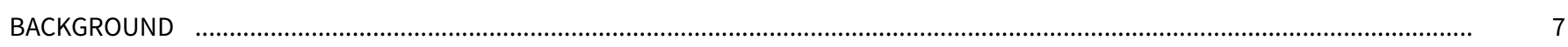

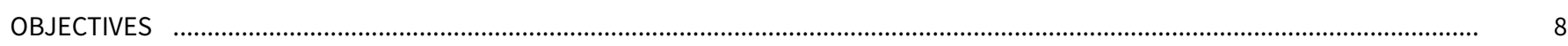

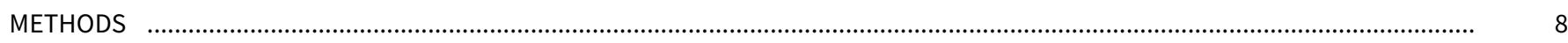

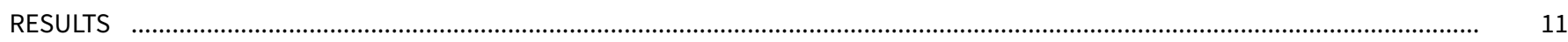

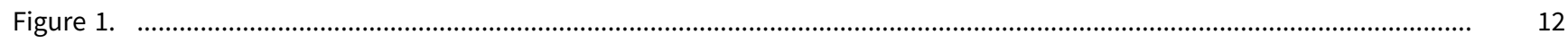

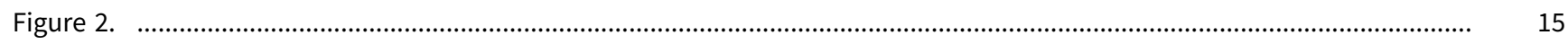

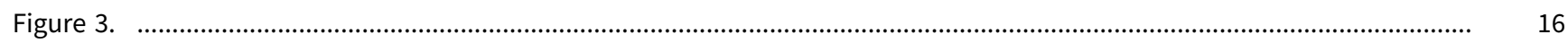

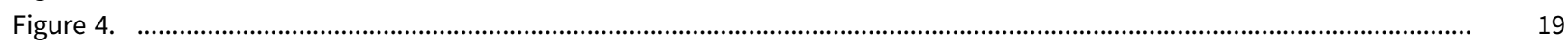

Figure 5.

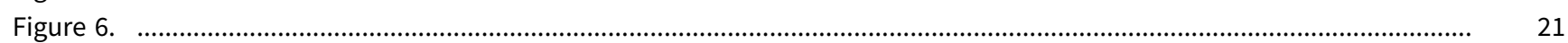

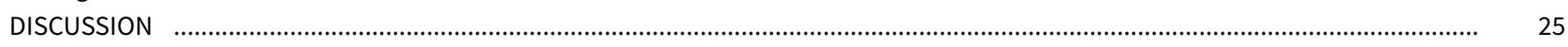

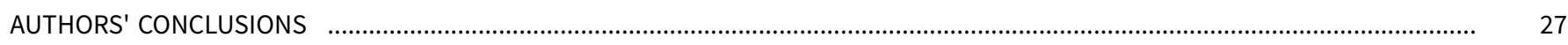

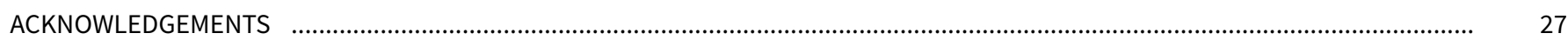

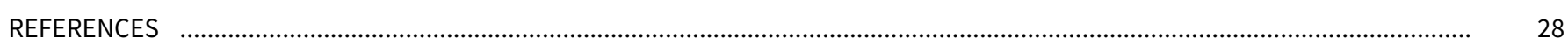

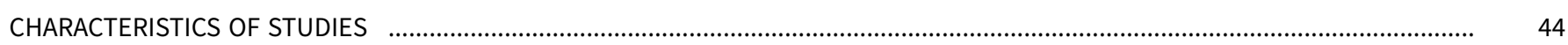

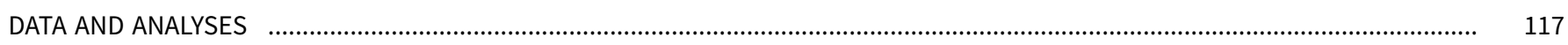

Analysis 1.1. Comparison 1: Educational or behavioural interventions for patients versus usual care, Outcome 1: Mean systolic 118

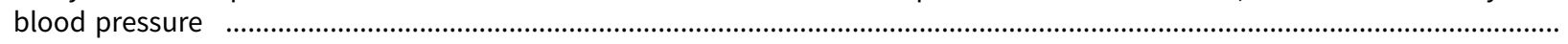

Analysis 1.2. Comparison 1: Educational or behavioural interventions for patients versus usual care, Outcome 2: Mean diastolic blood pressure

Analysis 1.3. Comparison 1: Educational or behavioural interventions for patients versus usual care, Outcome 3: Blood pressure target achievement

Analysis 1.4. Comparison 1: Educational or behavioural interventions for patients versus usual care, Outcome 4: Mean total cholesterol

Analysis 1.5. Comparison 1: Educational or behavioural interventions for patients versus usual care, Outcome 5: Total cholesterol target achievement

Analysis 1.6. Comparison 1: Educational or behavioural interventions for patients versus usual care, Outcome 6: Mean low density lipoprotein

Analysis 1.7. Comparison 1: Educational or behavioural interventions for patients versus usual care, Outcome 7: Mean high density lipoprotein

Analysis 1.8. Comparison 1: Educational or behavioural interventions for patients versus usual care, Outcome 8: Mean triglycerides

Analysis 1.9. Comparison 1: Educational or behavioural interventions for patients versus usual care, Outcome 9: Mean HbA1c .

Analysis 1.10. Comparison 1: Educational or behavioural interventions for patients versus usual care, Outcome 10: $\mathrm{HbA} 1 \mathrm{C}$ target achievement

Analysis 1.11. Comparison 1: Educational or behavioural interventions for patients versus usual care, Outcome 11: Mean BMI . Analysis 1.12. Comparison 1: Educational or behavioural interventions for patients versus usual care, Outcome 12: Proporation of participants with secondary stroke

Analysis 1.13. Comparison 1: Educational or behavioural interventions for patients versus usual care, Outcome 13: Number of secondary TIAs

Analysis 1.14. Comparison 1: Educational or behavioural interventions for patients versus usual care, Outcome 14: Number of myocardial infarctions

Analysis 1.15. Comparison 1: Educational or behavioural interventions for patients versus usual care, Outcome 15: Number of cardiovascular deaths

Analysis 2.1. Comparison 2: Organisational interventions versus usual care, Outcome 1: Mean systolic blood pressure ............ Analysis 2.2. Comparison 2: Organisational interventions versus usual care, Outcome 2: Mean diastolic blood pressure .......... Analysis 2.3. Comparison 2: Organisational interventions versus usual care, Outcome 3: Blood pressure target achievement ... Analysis 2.4. Comparison 2: Organisational interventions versus usual care, Outcome 4: Mean total cholesterol ...................... Analysis 2.5. Comparison 2: Organisational interventions versus usual care, Outcome 5: Total cholesterol target achievement . Analysis 2.6. Comparison 2: Organisational interventions versus usual care, Outcome 6: Mean low density lipoprotein 
Analysis 2.7. Comparison 2: Organisational interventions versus usual care, Outcome 7: Low density lipoprotein target achievement

Analysis 2.8. Comparison 2: Organisational interventions versus usual care, Outcome 8: Mean high density lipoprotein .......... Analysis 2.9. Comparison 2: Organisational interventions versus usual care, Outcome 9: High density lipoprotein target achievement

Analysis 2.10. Comparison 2: Organisational interventions versus usual care, Outcome 10: Mean triglycerides ........................ Analysis 2.11. Comparison 2: Organisational interventions versus usual care, Outcome 11: Triglyceride target achievement ... Analysis 2.12. Comparison 2: Organisational interventions versus usual care, Outcome 12: Mean HbA1C

Analysis 2.13. Comparison 2: Organisational interventions versus usual care, Outcome 13: $\mathrm{HbA} 1 \mathrm{C}$ target achievement ........... Analysis 2.14. Comparison 2: Organisational interventions versus usual care, Outcome 14: Mean BMI ................................... Analysis 2.15. Comparison 2: Organisational interventions versus usual care, Outcome 15: BMI target achievement ................ Analysis 2.16. Comparison 2: Organisational interventions versus usual care, Outcome 16: Mean Framingham cardiovascular risk score

Analysis 2.17. Comparison 2: Organisational interventions versus usual care, Outcome 17: Proportion of participants with secondary stroke or TIA

Analysis 2.18. Comparison 2: Organisational interventions versus usual care, Outcome 18: Number of secondary strokes ....... Analysis 2.19. Comparison 2: Organisational interventions versus usual care, Outcome 19: Number of secondary TIAs ........... Analysis 2.20. Comparison 2: Organisational interventions versus usual care, Outcome 20: Number of secondary TIA or stroke . Analysis 2.21. Comparison 2: Organisational interventions versus usual care, Outcome 21: Proportion of participants with secondary cardiovascular events

Analysis 2.22. Comparison 2: Organisational interventions versus usual care, Outcome 22: Number of secondary cardiovascular events

Analysis 2.23. Comparison 2: Organisational interventions versus usual care, Outcome 23: Number of myocardial infarctions .. Analysis 2.24. Comparison 2: Organisational interventions versus usual care, Outcome 24: Number of vascular deaths .......... ADDITIONAL TABLES

APPENDICES

WHAT'S NEW

HISTORY 
[Intervention Review]

\section{Interventions for improving modifiable risk factor control in the secondary prevention of stroke}

Bernadeta Bridgwood ${ }^{1}$, Kate E Lager ${ }^{1}$, Amit K Mistri², Kamlesh Khunti ${ }^{3}$, Andrew D Wilson ${ }^{1}$, Priya Modi4

1Department of Health Sciences, University of Leicester, Leicester, UK. 2Department of Cardiovascular Sciences, University of Leicester, Leicester, UK. ${ }^{3}$ Diabetes Research Centre, University of Leicester, Leicester, UK. ${ }^{4}$ Faculty of Medicine, Charles University, Prague, Czech Republic

Contact: Amit K Mistri, akm17@le.ac.uk.

Editorial group: Cochrane Stroke Group.

Publication status and date: Edited (no change to conclusions), published in Issue 6, 2022.

Citation: Bridgwood B, Lager KE, Mistri AK, Khunti K, Wilson AD, Modi P. Interventions for improving modifiable risk factor control in the secondary prevention of stroke. Cochrane Database of Systematic Reviews 2018, Issue 5. Art. No.: CD009103. DOI: 10.1002/14651858.CD009103.pub3.

Copyright @ 2022 The Cochrane Collaboration. Published by John Wiley \& Sons, Ltd.

\section{A B S T R A C T}

\section{Background}

People with stroke or transient ischaemic attack (TIA) are at increased risk of future stroke and other cardiovascular events. Stroke services need to be configured to maximise the adoption of evidence-based strategies for secondary stroke prevention. Smoking-related interventions were examined in a separate review so were not considered in this review. This is an update of our 2014 review.

\section{Objectives}

To assess the effects of stroke service interventions for implementing secondary stroke prevention strategies on modifiable risk factor control, including patient adherence to prescribed medications, and the occurrence of secondary cardiovascular events.

\section{Search methods}

We searched the Cochrane Stroke Group Trials Register (April 2017), the Cochrane Effective Practice and Organisation of Care Group Trials Register (April 2017), CENTRAL (the Cochrane Library 2017, issue 3), MEDLINE (1950 to April 2017), Embase (1981 to April 2017 ) and 10 additional databases including clinical trials registers. We located further studies by searching reference lists of articles and contacting authors of included studies.

\section{Selection criteria}

We included randomised controlled trials (RCTs) that evaluated the effects of organisational or educational and behavioural interventions (compared with usual care) on modifiable risk factor control for secondary stroke prevention.

\section{Data collection and analysis}

Four review authors selected studies for inclusion and independently extracted data. The quality of the evidence as 'high', 'moderate', 'low' or 'very low' according to the GRADE approach (GRADEpro GDT).Three review authors assessed the risk of bias for the included studies. We sought missing data from trialists.The results are presented in 'Summary of findings' tables.

\section{Main results}

The updated review included 16 new studies involving 25,819 participants, resulting in a total of 42 studies including 33,840 participants. We used the Cochrane risk of bias tool and assessed three studies at high risk of bias; the remainder were considered to have a low risk of bias. We included 26 studies that predominantly evaluated organisational interventions and 16 that evaluated educational and behavioural 
interventions for participants. We pooled results where appropriate, although some clinical and methodological heterogeneity was present.

Educational and behavioural interventions showed no clear differences on any of the review outcomes, which include mean systolic and diastolic blood pressure, mean body mass index, achievement of $\mathrm{HbAlc}$ target, lipid profile, mean HbAlc level, medication adherence, or recurrent cardiovascular events. There was moderate-quality evidence that organisational interventions resulted in improved blood pressure control, in particular an improvement in achieving target blood pressure (odds ratio (OR) $1.44,95 \%$ confidence interval $(\mathrm{Cl}) 1.09$ to1.90; 13 studies; 23,631 participants). However, there were no significant changes in mean systolic blood pressure (mean difference (MD), $-1.58 \mathrm{mmHg} 95 \% \mathrm{Cl}-4.66$ to $1.51 ; 16$ studies; 17,490 participants) and mean diastolic blood pressure ( $\mathrm{MD}-0.91 \mathrm{mmHg} 95 \% \mathrm{Cl}-2.75$ to 0.93 ; 14 studies; 17,178 participants). There were no significant changes in the remaining review outcomes.

\section{Authors' conclusions}

We found that organisational interventions may be associated with an improvement in achieving blood pressure target but we did not find any clear evidence that these interventions improve other modifiable risk factors (lipid profile, HbA1c, medication adherence) or reduce the incidence of recurrent cardiovascular events. Interventions, including patient education alone, did not lead to improvements in modifiable risk factor control or the prevention of recurrent cardiovascular events.

\section{PLAIN LANGUAGE SUMMARY}

Healthcare interventions for reducing the risk of future stroke in people with previous stroke or transient ischaemic attack (TIA)

\section{Review question}

How effective are healthcare interventions for preventing a recurrent stroke or other cardiovascular events in people who have had a stroke or a transient ischaemic attack (TIA: also known as a mini-stroke)?

\section{Background}

Stroke and TIA are diseases caused by interruptions in the blood supply to the brain. People who experience a stroke or TIA are at risk of future stroke. Several medications and lifestyle changes can be used to lower stroke risk by improving the control of modifiable risk factors such as blood pressure, blood fats, being overweight, raised blood sugar, and the use of preventive medications. These risk factors are often not managed effectively following a stroke or TIA. It is important to identify healthcare interventions that can help prevent stroke by improving these risk factors. Interventions in this review targeted patients or clinicians, or both (aimed at education or changing behaviour, or both); and organisations (e.g. changing the way services were provided).

This is an update of our review published in 2014.

\section{Search date}

We searched for studies up to April 2017.

\section{Study characteristics}

This updated review included 16 new studies involving 25,819 participants, resulting in a total of 42 studies including 33,840 with stroke or TIA whose average age ranged from 60 to 74.3 years. Most studies took place in primary care or community settings. Sixteen studies involved educational or behavioural interventions for participants and 26 studies mostly involved organisational interventions. Most interventions lasted for between three and 12 months, with follow-up from three months up to three years.

\section{Key results}

Changes to healthcare services that looked at patient education or behaviour only, without any alterations in the organisation of patient care, showed no clear evidence of improvements in risk factors for stroke. Changes in the organisation of healthcare services resulted in improvements in blood pressure control. The effects of these interventions on changes in blood fats, blood sugar, body weight, or use of medicines were not conclusive.

We identified 24 ongoing studies suggesting that research in this area is increasing.

\section{Quality of the evidence}

The available evidence was assessed as moderate- or low-quality because of variations in methods used and results reported. 


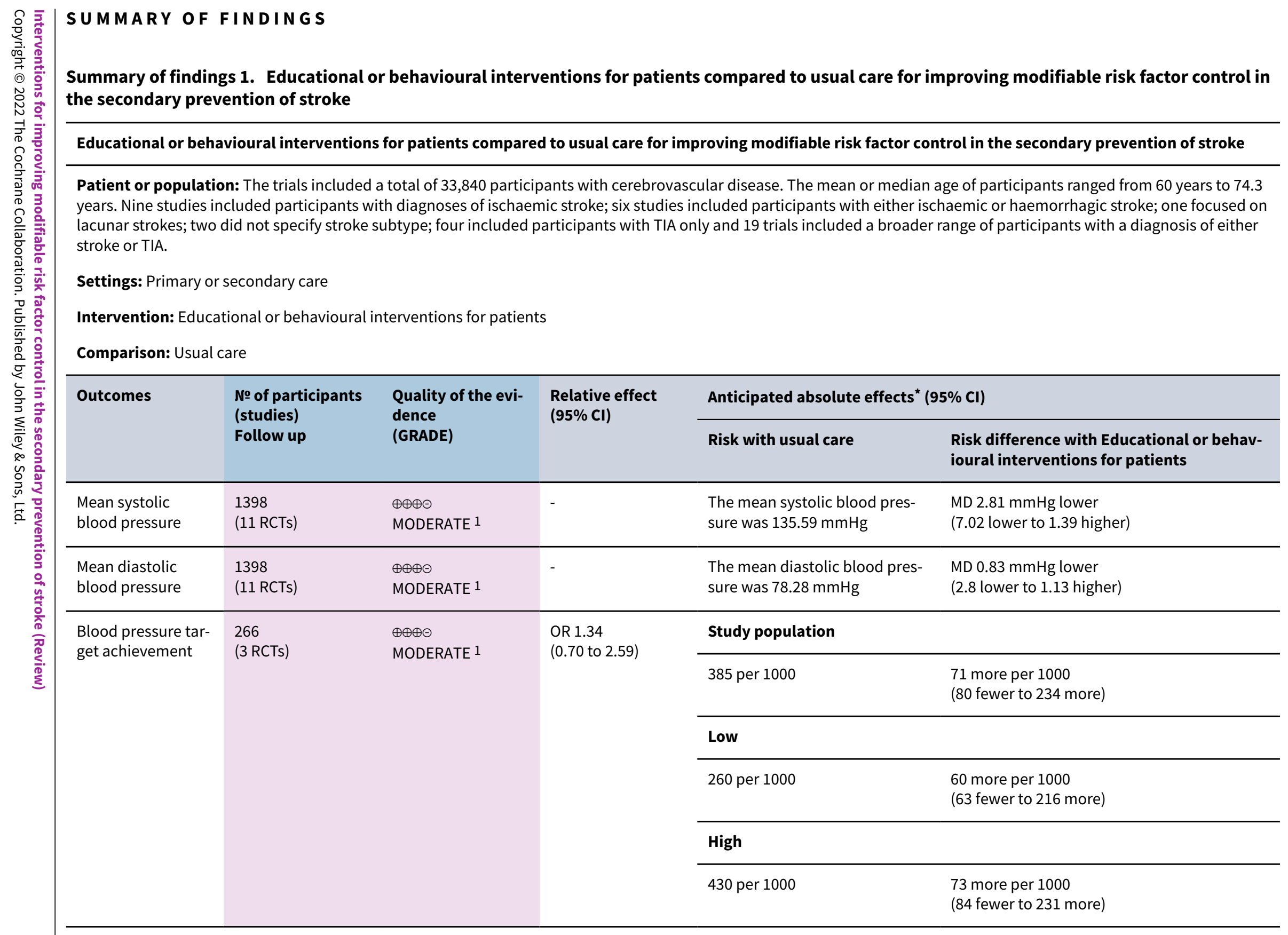




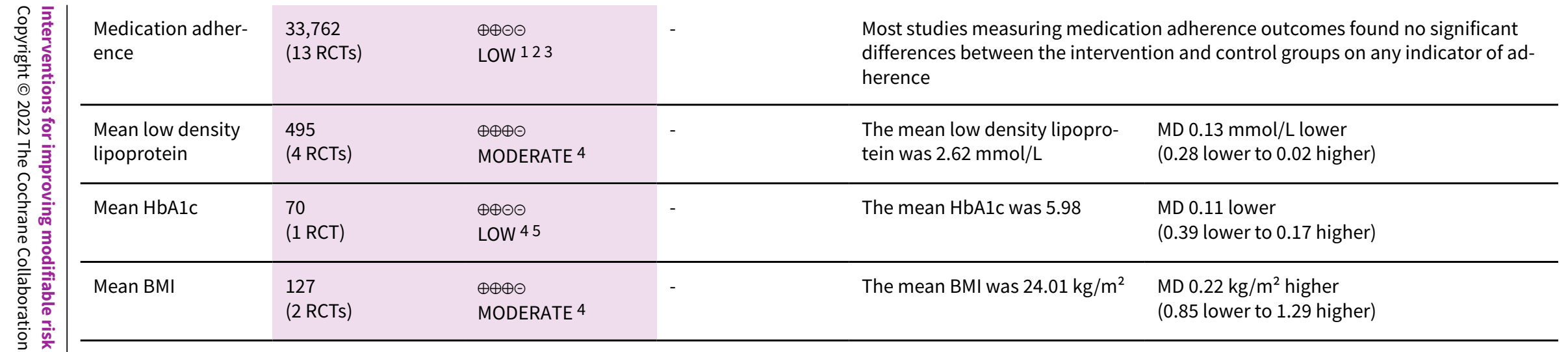

${ }^{*}$ The risk in the intervention group (and its 95\% confidence interval) is based on the assumed risk in the comparison group and the relative effect of the intervention (and its $95 \% \mathrm{Cl})$.

Cl: Confidence interval; OR: Odds ratio;

\section{GRADE Working Group grades of evidence}

High quality: We are very confident that the true effect lies close to that of the estimate of the effect

Moderate quality: We are moderately confident in the effect estimate: The true effect is likely to be close to the estimate of the effect, but there is a possibility that it is substantially different

Low quality: Our confidence in the effect estimate is limited: The true effect may be substantially different from the estimate of the effect

Very low quality: We have very little confidence in the effect estimate: The true effect is likely to be substantially different from the estimate of effect

1 The methods used in these studies were heterogenous which made these difficult to directly correlate

2 Contains at least one study that scores 'high' using the Cochrane risk analysis and thus down graded by one level

3 Results were inconsistent across the studies

4 Secondary outcome

5 One study provided evidence for this outcome

\section{Summary of findings 2. Organisational interventions compared to usual care for improving modifiable risk factor control in the secondary} prevention of stroke

Organisational interventions compared to usual care for improving modifiable risk factor control in the secondary prevention of stroke

Patient or population: The trials included a total of 33,840 participants with cerebrovascular disease. The mean or median age of participants ranged from 60 years to 74.3 years. Nine studies included participants with diagnoses of ischaemic stroke; six studies included participants with either ischaemic or haemorrhagic stroke; one focused on lacunar strokes; two did not specify stroke subtype; four included participants with TIA only and 19 trials included a broader range of participants with a diagnosis of either stroke or TIA.

Settings: Primary or secondary care 


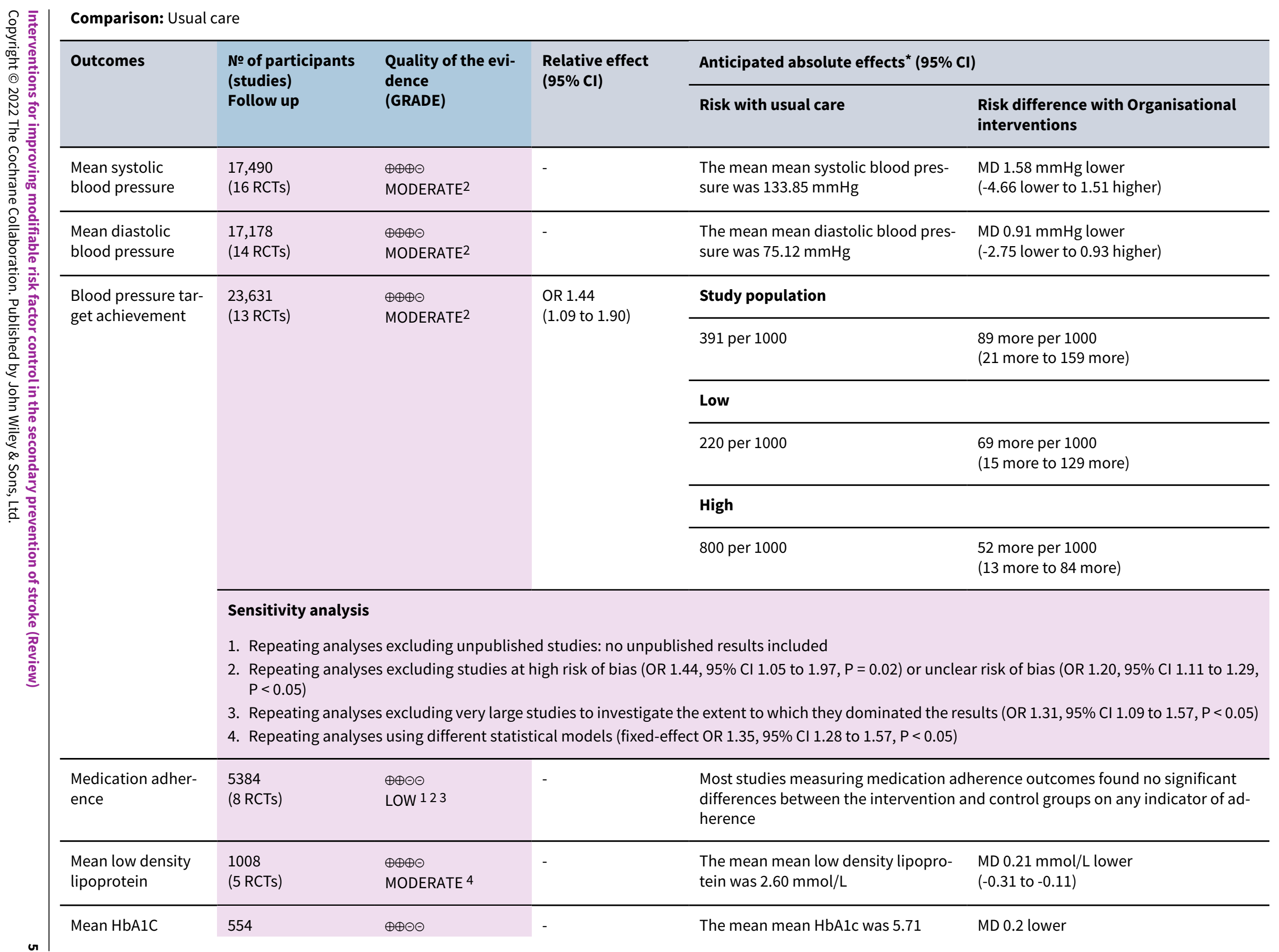




LOW 34

$\mathrm{m}^{2}$

(-1.24 to 0.30$)$

${ }^{*}$ The risk in the intervention group (and its $95 \%$ confidence interval) is based on the assumed risk in the comparison group and the relative effect of the intervention (and its $95 \% \mathrm{Cl}$ ).

Cl: Confidence interval; RR: Risk ratio; OR: Odds ratio;

\section{GRADE Working Group grades of evidence}

High quality: We are very confident that the true effect lies close to that of the estimate of the effect

Moderate quality: We are moderately confident in the effect estimate: The true effect is likely to be close to the estimate of the effect, but there is a possibility that it is substantially different

Low quality: Our confidence in the effect estimate is limited: The true effect may be substantially different from the estimate of the effect

Very low quality: We have very little confidence in the effect estimate: The true effect is likely to be substantially different from the estimate of effect

1 One included study did not include an explanation of blinding

2 The methods and outcome measures used in these studies were heterogenous which made these difficult to directly correlate

3 One study deemed high risk when assessed using Cochrane risk of bias tool Contains at least one study thus down graded by one level

4 The methods used in these studies were heterogenous which made these difficult to directly correlate 


\section{B A C K G R O U N D}

\section{Description of the condition}

Stroke is defined as a rapidly developing neurological deficit of presumed vascular origin, lasting for over 24 hours or leading to death (WHO 1978). Transient ischaemic attack (TIA) is an expression used traditionally to describe comparable neurological deficits lasting for fewer than 24 hours (Albers 2002). More recently, a new definition of TIA has been proposed, omitting the arbitrary 24-hour time frame and identifying a TIA as a "transient episode of neurological dysfunction caused by focal brain, spinal cord, or retinal ischaemia, without acute infarction" (Easton 2009).

The World Health Organization (WHO) has reported that cerebrovascular disease (stroke) is the second leading cause of mortality and disease burden among adults aged 60 years and over (Feigin 2014; Feigin 2016; Fourth SSNAP Annual Report 2016/17; Stroke Association 2018; WHO 2017). Following a TIA or minor stroke people have a $5.1 \%$ risk of stroke recurrence in the next year (Amarenco 2016). Long-term cohort studies have demonstrated that the risk of cardiovascular events remains high for at least 10 years after stroke or TIA (Touze 2005; Van Wijk 2005). Secondary prevention strategies aim to prevent recurrent events by improving modifiable risk factor control. National stroke guidelines identify clinical conditions (hypertension, hyperlipidaemia, atrial fibrillation, diabetes, and obesity) and lifestyle factors (smoking, physical inactivity, unhealthy diet, and excess alcohol consumption) as significant modifiable risk factors that should be targeted for secondary prevention (Canadian Stroke Best Practices 2017; ESO 2008; Kernan 2014; National Stroke Foundation 2017; SIGN 2008; Stroke Audit 2016). The strength of evidence for benefit from modifying risk factors varies: there is direct clinical trial evidence for treatment of hypertension and raised lipids, anti-platelet drugs, anticoagulation for atrial fibrillation, surgery for carotid stenosis and, more recently, insulin resistance (Kernan 2016). The evidence for lifestyle interventions such as improving control of diabetes, weight loss, smoking cessation, and alcohol reduction relies on observational studies (Hankey 2014).

\section{Description of the intervention}

For the purposes of this review, we considered stroke services to include all services responsible for providing acute and followup care to people with stroke and TIA. Stroke services exist as part of diverse healthcare systems, with specific treatment goals varying according to national clinical guidelines. Acute stroke services include organised inpatient (stroke unit) care and specialist TIA clinics (RCP 2016; Stroke Unit Trialists' Collaboration 2013). Recommendations for secondary prevention can be initiated as part of a co-ordinated treatment programme during acute hospitalisation (Ovbiagele 2004). However, primary care services are well placed to monitor patient risk factors, encourage lifestyle change and review secondary prevention medications on an ongoing basis (RCP 2016). Primary care aims to be characterised by person-centred, comprehensiveness, continuity of care, and community participation (Starfield 2002; WHO 2008). Social care services and voluntary sector organisations can also work in partnership with primary care to deliver healthy living support (NAO 2005). Stroke service interventions are considered complex interventions since they often contain several interacting components and may require complex behaviours, organisational change, or the assessment of numerous outcome measures (Craig 2008; Redfern 2008).

\section{How the intervention might work}

Stroke services addressing secondary prevention aim to improve patient adherence with medication regimens and lifestyle advice. Several classes of medication reduce stroke incidence by modifying cardiovascular risk. For example, long-term antiplatelet medication in those with a history of stroke or TIA is associated with a significant $25 \%$ reduction in secondary vascular events (Antithrombotic Trialists' Collaboration 2002; Barber 2016). Similarly, antihypertensive and statin medications are associated with improvements in secondary prevention (Collins 2016; Ettehad 2016; Logue 2015; Preiss 2015; Sundström 2014;). Meta-analyses report that moderate to high physical activity (Bennett 2017; Fan 2017), moderate alcohol consumption (Holmes 2014; Reynolds 2003), reduction of salt intake (Aburto 2013; He 2013), and specific dietary changes (He 2004; He 2006) can also facilitate stroke prevention and cardiovascular risk reduction. An international case-control study identified five modifiable risk factors accounting for $83 \%$ of the population attributable risk (PAR) for stroke (O'Donnell 2010; Perk 2012). Targeting multiple risk factors may have additive benefits for secondary prevention, for example, a modelling study predicted that a $80 \%$ cumulative risk reduction in recurrent vascular events could be achieved by combining dietary modification, exercise, aspirin, a statin, and an antihypertensive agent (Hackam 2007; Perk 2012).

\section{Why it is important to do this review}

Most people with stroke have at least one cardiovascular risk factor and hypertension, hyperlipidaemia, diabetes, smoking, and obesity are often inadequately managed during follow-up (Hankey 2014; Herttua 2016; Kernan 2014; Perreault 2012; Xu 2017). Although the effectiveness of secondary prevention medications is well-established, non-treatment rates for antithrombotic, antihypertensive, and statin therapies remain high after stroke (Hankey 2014; Raine 2009) and TIA (Lager 2012). This includes a large proportion due to behavioural factors such as smoking and low physical activity (Feigin 2016). Only 31\% of people with stroke and $35 \%$ of people with TIA receive combination treatment with all three medication classes (Ramsay 2007). Furthermore, adherence to secondary prevention medications falls progressively as time since the primary stroke elapses (Glader 2010). As strategies for stroke prevention are not optimally implemented, substantial benefits stand to be gained from improving the use of evidencebased interventions (Goldstein 2008).

Several studies have revealed inequalities in the provision of stroke care with older people being less likely to receive or adhere to secondary prevention medication (De Schryver 2005; Raine 2009; Ramsay 2007). Similarly, people with stroke who have more severe disability (Barthel scores of 14 or less) are less likely to receive appropriate secondary prevention than those with mild disability (Barthel score 15 to 20) (Rudd 2004). Ethnic groups are also reported to differ with respect to patterns in behavioural risk factors for stroke (Dundas 2001). These subgroups of people may require targeted interventions to improve risk factor control.

Service interventions used for other conditions, particularly secondary prevention of ischaemic heart disease, may be relevant to the secondary prevention of stroke (Buckley 2010; Kernan 2014). 
However, more direct evidence is needed to guide improvements in follow-up care after stroke or TIA. For example, stroke commonly results in cognitive impairments or physical disabilities that are likely to influence both intervention design and outcomes. To date, there are no systematic reviews that have considered the impact of stroke service interventions on cardiovascular risk factor control or adherence to secondary prevention medications. An assessment of the quality and outcomes of previous studies in this field will inform the development of new interventions.

\section{OB JECTIVES}

To assess the effects of stroke service interventions for implementing secondary stroke prevention strategies on modifiable risk factor control, including patient adherence to prescribed medications, and the occurrence of secondary cardiovascular events.

\section{METHOD S}

\section{Criteria for considering studies for this review}

\section{Types of studies}

We included published or unpublished randomised controlled trials (RCTs) with a minimum follow-up of three months after the start of the intervention. Parallel group trials, cluster-randomised trials and cross-over trials were eligible for inclusion in the review.

\section{Types of participants}

We included adults (aged 18 years and over) with a confirmed diagnosis of ischaemic stroke, haemorrhagic stroke, or transient ischaemic attack (TIA).

\section{Types of interventions}

For the purposes of this review, we defined stroke service educational or organisational interventions as alternative models of care that are implemented to improve patient outcomes following stroke or TIA. We included stroke service interventions that were intended to improve modifiable risk factor control. We focused on interventions that aimed to improve modifiable risk factor control through increased adherence to existing recommendations for secondary stroke prevention (e.g. recommendations in international stroke guidelines). We did not consider smoking-related interventions which have been extensively reported elsewhere (Critchley 2012; Stead 2013a; Stead 2013b; Stead 2017; Taylor 2017; Whittaker 2016).

Following EPOC guidelines (EPOC 2015) we considered the following intervention categories (pre-specified in the review protocol). Because educational and organisational interventions differ in their theoretical frameworks, the protocol stated these would be analysed separately (Lager 2011).

- Educational and behavioural interventions for stroke patients.

- Educational and behavioural interventions for stroke service providers.

- Organisational interventions (subdivided into the following categories developed by Wensing 2006):

- revision of professional roles, e.g. involvement of nonphysician staff in prevention clinics;
- collaboration between multidisciplinary teams, e.g. interventions promoting effective liaison between primary and secondary care teams;

- integrated care services, e.g. disease and case management programs where patient care follows protocols for screening, education and treatment or monitoring;

- knowledge management systems, e.g. computerised decision support on medication prescribing, shared medical records;

- quality management, e.g. guideline and protocol development;

- financial incentives, e.g. the UK Quality and Outcomes Framework (NHS 2014).

We excluded interventions that were intended to improve physical rehabilitation or knowledge of stroke in general, surgical interventions, and interventions testing new pharmacological therapies. We also excluded exercise training programs for people with stroke or TIA which are the subject of other Cochrane Reviews (MacKay-Lyons 2013; Saunders 2016).

\section{Types of outcome measures}

\section{Primary outcomes}

- Target achievement or mean reductions, or both, for blood pressure, lipid profile (total cholesterol), high density lipoprotein (HDL), low density lipoprotein (LDL), triglycerides (TG), glycaemic control (HbA1c), body mass index (BMI), or validated cardiovascular risk score.

- Any indicator of patient adherence to secondary prevention medications, e.g. self-reported medication adherence or medication persistence, medication possession, individual patient data on prescriptions, pharmacy claims, electronic monitoring, drug tracers in blood or urine. Secondary prevention medications include those to lower causal risk factors (blood pressure, lipids, etc.) as well as antithrombotics to directly reduce the risk of a cerebrovascular event.

\section{Secondary outcomes}

- Secondary cardiovascular events: stroke, myocardial infarction, or vascular death or composites. Because this review focused on long-term prevention, we did not include surgical interventions for carotid stenosis nor identification and management of atrial fibrillation. We also excluded other more recently identified risk factors, such as insulin resistance.

\section{Search methods for identification of studies}

See the 'Specialised register' section in the Cochrane Stroke Group module. We searched for trials in all languages and arranged for translation of relevant papers where necessary.

\section{Electronic searches}

We searched the following electronic databases to identify relevant trials:

- Cochrane Stroke Group Trials Register (to April 2017);

- Cochrane Effective Practice and Organisation of Care Group Trials Register (to April 2017); 
- Cochrane Central Register of Controlled Trials (CENTRAL; 2017, Issue 5) in the Cochrane Library (searched May 2017) (Appendix 1);

- MEDLINE in Ovid (1950 to April 2017) (Appendix 2);

- Embase in Ovid (1981 to April 2017) (Appendix 3);

- CINAHL in EBSCO (Cumulative Index to Nursing and Allied Health Literature; 1982 to April 2017) (Appendix 4);

- AMED in Ovid (Allied and Complementary Medicine Database; 1985 to April 2017) (Appendix 5);

- British Nursing Index (BNI) in Ovid (1985 to April 2017) (Appendix 6);

- Web of Science Conference Proceedings Citation Index - Science (1970 to April 2017) (Appendix 7); and

- Bibliomap (health promotion research) (April 2017) (www.eppi.ioe.ac.uk/webdatabases/Intro.aspx?ID=7).

We also searched the following databases of ongoing trials and grants registers:

- US National Institutes of Health Ongoing Trials Register (ClinicalTrials.gov (www.clinicaltrials.gov; searched April 2017) (Appendix 8);

- ISRCTN Registry (www.isrctn.com; searched April 2017) (Appendix 9);

- Stroke Trials Registry (www.strokecenter.org/trials/; searched April 2017) (Appendix 10); and

- World Health Organization (WHO) International Clinical Trials Registry Platform (www.apps.who.int/trialsearch/; searched April 2017) (Appendix 11)

\section{Searching other resources}

We used the Science Citation Index Cited Reference Search to search for studies citing included trials. We also checked the reference lists of included trials, relevant systematic reviews, and relevant meta-analyses. We contacted authors and trialists involved in included trials to facilitate identification of ongoing trials and unpublished studies.

\section{Data collection and analysis}

\section{Selection of studies}

For the previous version of this review, two review authors ( $K L$ and a second review author) independently assessed the titles, abstracts and keywords of all records retrieved from the electronic searches and excluded obviously irrelevant studies (Lager 2014). We resolved any disagreements regarding study eligibility by discussion among all review authors. For this search update in April 2017, two review authors ( $B B$ and $A W$ ) undertook the same process, identifying relevant studies published since the original review. A third author (PM) validated the results and edited the review. We obtained the full texts of the remaining studies and two review authors independently selected studies for inclusion based on the following criteria.

\section{The study:}

- was an RCT;

- restricted participants to people with TIA or stroke, or reported outcomes separately for TIA or stroke patient subgroups;

- evaluated a stroke service intervention;
- stated or clearly implied that the intention of an intervention was to improve modifiable risk factor control;

- assessed one or more of the defined outcome measures; and

- did not include physical rehabilitation programs, new pharmacological therapies, surgical procedures, exercise training programmes, or educational programmes intended to improve knowledge of stroke in general.

\section{Data extraction and management}

For the previous version of this review, two review authors independently extracted outcome data for each eligible trial using a pre-specified data extraction form (Lager 2014). One review author extracted data for all eligible studies (KL) and a second review author (AKS and $\mathrm{VH}$ ) independently repeated data extraction for each study. We resolved disagreements by discussion to reach consensus, with review authors referring back to the original article. For this update, this method was repeated by BB, AW and PM respectively.

We recorded the following information for each study.

- General information: published or unpublished, title, authors, journal or source, publication date, country of origin, publication language.

- Study methods: unit of randomisation (and method), allocation concealment (and method), blinding (outcome assessors), validation of questionnaires.

- Participants: sampling (random or convenience), place of recruitment, total sample size, numbers randomised, inclusion criteria, exclusion criteria, demographic characteristics (age, gender, ethnicity, socio-economic or socio-demographic status), disability (modified Rankin score, Barthel score), comorbidities, similarity between groups at baseline, dropout and withdrawal rates.

- Intervention details: components, length, frequency, location, mode of delivery, personnel responsible for delivery, timing post-stroke, details of control protocol.

- Outcomes: pre-specified outcomes (see Selection of studies), follow-up intervals from start of intervention, units of measurement, missing data.

- Results: results for pre-specified outcomes, number of participants assessed, method of analysis (intention-to-treat analysis, per protocol analysis).

- Intervention category: pre-specified in the review protocol.

\section{Assessment of risk of bias in included studies}

Three review authors (KL, BB, AW) independently assessed the risk of bias for each included study, using the 'Risk of bias' tool described in the Cochrane Handbook for Systematic Reviews of Interventions (Higgins 2011a). We resolved any disagreements by discussion. We assessed the risk of bias according to the following domains.

- Random sequence generation.

- Allocation concealment.

- Blinding of participants and personnel.

- Blinding of outcome assessment.

- Incomplete outcome data.

- Selective outcome reporting. 


\section{- Other bias.}

We graded the risk of bias for each domain as of high, low, or unclear risk of bias and entered this information into the 'Risk of bias' table produced for each study in the Characteristics of included studies section, along with the reason for each decision. We contacted study authors to retrieve missing information. If study authors did not provide the requested information, we recorded the relevant items on the risk of bias assessment as 'unclear'.

We summarised the risk of bias according to the following criteria (Higgins 2011a).

- Low risk of bias: low risk of bias for all domains.

- Unclear risk of bias: unclear risk of bias for one or more domains.

- High risk of bias: high risk of bias for one or more domains.

\section{Measures of treatment effect}

A mixture of continuous outcomes and dichotomous outcomes were reported by studies included in this review. Where possible, we reported data in terms of mean difference (MD) and 95\% confidence interval $(\mathrm{Cl})$ for continuous data. For dichotomous data, we reported risk ratios (RR) or odds ratios (OR) and $95 \% \mathrm{Cls}$. If individual studies reported continuous and dichotomous data for the same outcome, we included both variables in the review. We used RevMan 5 to carry out statistical analyses (RevMan 2014).

\section{Unit of analysis issues}

We analysed cluster-RCTs by reporting effect estimates from analyses that accounted for the cluster design. Where necessary, we calculated effective sample sizes for cluster-RCTs and combined these with parallel RCTs in meta-analyses (Higgins 2011b). When examining recurrent events we aimed to analyse the number of people with one or more events rather than number of events. Where studies included repeated measurements for participants at several time points, we reported the outcomes recorded at the end of the study per protocol.

\section{Dealing with missing data}

We proposed to contact study authors if necessary to request any missing data and to input missing summary data (e.g. standard deviations) based on recommendations in the Cochrane Handbook for Systematic Reviews of Interventions (Deeks 2011; Higgins 2011b). There was an apparent inconsistency with the standard deviation values reported for Mackenzie 2013. We attempted to contact the author to clarify; however, we did not receive a response, so we used the published standard deviation values.

\section{Assessment of heterogeneity}

We identified heterogeneity from forest plots using the $\mathrm{Chi}^{2}$ test and a significance level of alpha $=0.1$. We also quantified heterogeneity using the $\mathrm{I}^{2}$ statistic, where $\mathrm{I}^{2}$ values of $50 \%$ or more indicate a substantial level of heterogeneity (Higgins 2002; Higgins 2003). Where appropriate, we assessed possible sources of heterogeneity using sensitivity analyses.

\section{Assessment of reporting biases}

We used funnel plots to assess publication bias.

\section{Data synthesis}

Included studies were heterogeneous in terms of interventions, settings, participant characteristics, and outcome measurements. Where there were sufficient comparable data we combined results for each outcome to give an overall estimate of treatment effect. We conducted meta-analyses separately for each intervention category to reduce clinical heterogeneity among the studies that were combined to produce pooled estimates using random-effects models. We pre-specified intervention categories in the review protocol. Where meta-analysis was not possible or appropriate, we presented results as a qualitative synthesis of intervention effects.

\section{Subgroup analysis and investigation of heterogeneity}

We planned to analyse outcomes according to the following subgroups.

- Participant age (under 65 years, 65 years and over).

- Condition (ischaemic stroke, haemorrhagic stroke, or TIA).

- Stroke severity (e.g. according to the National Institute of Health Stroke Scale (NIHSS)) or disability (e.g. according to the Barthel score or modified Rankin Score (mRS)).

- Specific risk factor management strategy (e.g. blood pressure lowering interventions).

However, subgroup analyses were not possible because relevant data were not available from the included studies. We were, however, able to undertake subgroup analysis for studies involving multidisciplinary team members.

\section{Sensitivity analysis}

We undertook sensitivity analysis for achievement of blood pressure targets using the following criteria.

- Repeating analyses excluding unpublished studies.

- Repeating analyses excluding studies at high or unclear risk of bias.

- Repeating analyses excluding very large studies to investigate the extent to which they dominated the results.

- Repeating analyses using different measures of effect size (risk difference, odds ratio etc.) and different statistical models (fixedeffect and random-effects models).

\section{Summary of findings and assessment of the certainty of the evidence}

We used GRADEpro GDT to import data from Review Manager 5 (RevMan 2014) in order to create 'Summary of findings' tables. Within these tables, we presented a summary of the evidence for educational and behavioural interventions for participants receiving treatment compared with those in the control group for secondary stroke prevention (Summary of findings 1), and organisational interventions for participants receiving treatment compared with those in the control group for secondary stroke prevention (Summary of findings table 2). We included the following outcomes: mean systolic and diastolic blood pressure, blood pressure target achievement, medication adherence, mean low density lipoprotein, mean HbAlc and mean BMI.

We justified judgements about the quality of the evidence (high, moderate, low, or very low) according to the GRADE approach (Higgins 2011c), which we documented and incorporated into the 
reporting of results for each outcome. The quality of evidence could be downgraded by one level (serious concern) or two levels (very serious concerns) due to concerns raised within: risk of bias; inconsistency (unexplained heterogeneity, inconsistency of results); indirectness (indirect population, intervention, control, outcomes) and due to imprecision (wide Cls, single trials). Grade outcomes are presented in the 'Summary of findings' tables (Summary of findings 1; Summary of findings 2).

\section{RE S U L T S}

\section{Description of studies}

See: Characteristics of included studies; Characteristics of excluded studies; Characteristics of studies awaiting classification; Characteristics of ongoing studies

\section{Results of the search}

We carried out searches in April 2013 and updated the search in April 2017 and identified a total of 19,147 records after the removal of duplicates (Figure 1). Title and abstract screening identified 171 studies (82 in the first review (Lager 2014) and 89 in this update, consisting of 428 records collectively) that were potentially eligible for this review. 
Figure 1. Study flow diagram.

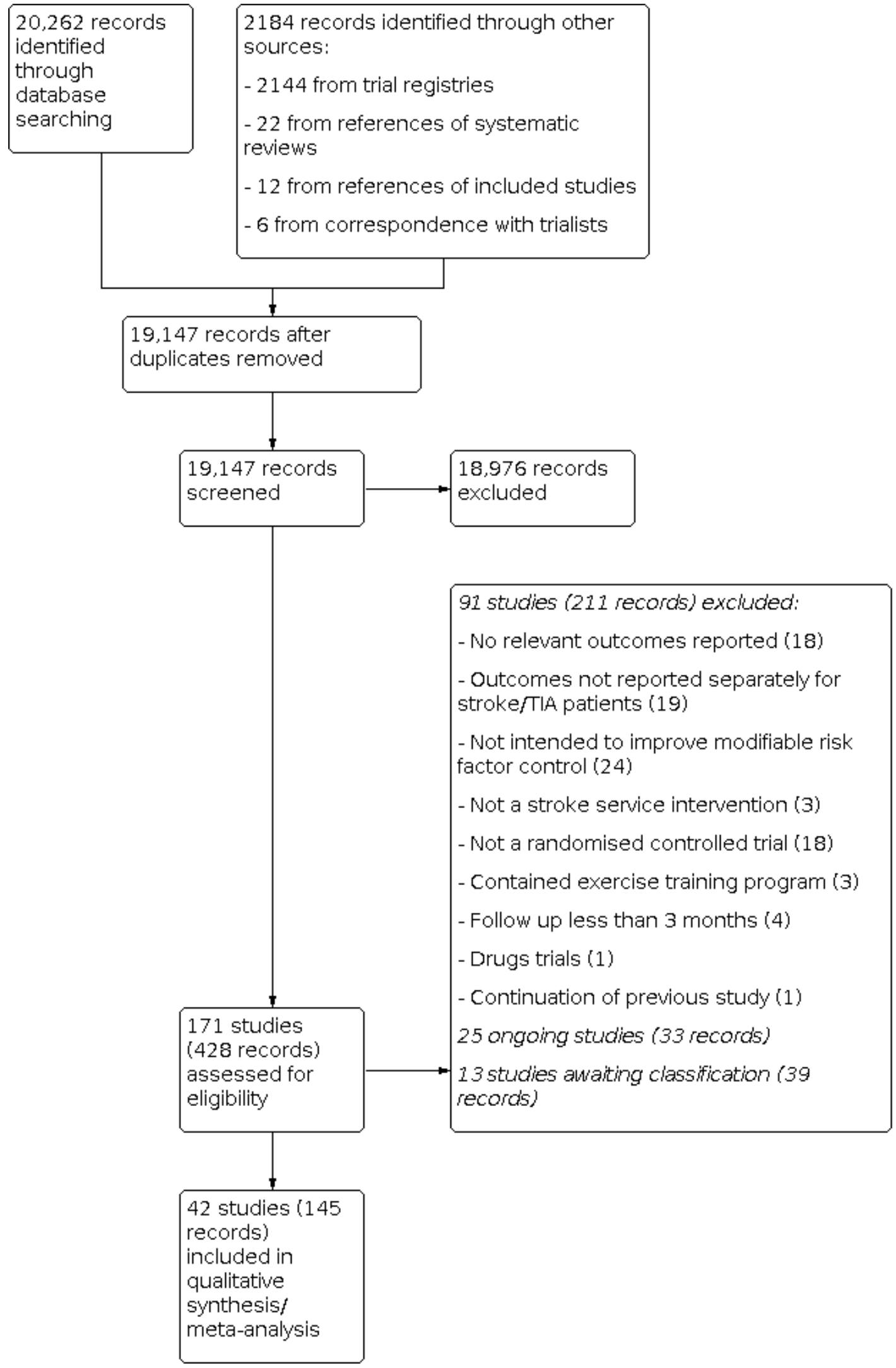


We found 10 potentially eligible studies that reported collective outcome data for participants with a broad range of cardiovascular diseases (Amariles 2012; Brotons 2011; Evans 2010; Goessens 2006; Ma 2009; McManus 2014; Palanco 2011; Spassova 2016; Strandberg 2006; Vernooij 2012). We contacted study authors to request outcome data separately for participants with stroke and transient ischaemic attack (TIA). We received responses from four study authors who provided unpublished outcome data for participants with stroke and TIA; these studies were included in the review (Brotons 2011; Evans 2010; Jönsson 2014; McManus 2014). The authors of one study reported that separate outcome data for participants with stroke and TIA were unavailable (Vernooij 2012). The authors of six studies did not respond to requests for additional data and these studies were excluded from the review (Amariles 2012; Goessens 2006; Ma 2009; Palanco 2011; Spassova 2016; Strandberg 2006).

We identified a further 47 studies of potential relevance to this review, if unpublished outcome data were available. We therefore attempted to obtain information about these studies by emailing the main study contacts. Seven authors supplied unpublished data, for example blood pressure or body mass index (BMI). We included these studies in the review (Eames 2013; Flemming 2013; Lowrie 2010; Jönsson 2014; McManus 2014; O'Carroll 2011; Slark 2013).

\section{Included studies}

We added 16 new studies (25,819 participants), to the 26 studies (8021 participants) in the previous version of the review, resulting in a total of 42 studies including 33,840 participants in this update. Of these 36 used a parallel group design (Adie 2010; Allen 2002; Allen 2009; MIST 2014; Boter 2004; Boysen 2009; Chanruengvanich 2006; Chiu 2008; Damush 2015; Eames 2013; Ellis 2005; Evans 2010; Flemming 2013; Hanley 2015; Hedegaard 2014; Hornnes 2011; Nailed Stroke 2010; Jönsson 2014; Joubert 2009; Kerry 2013; Kim 2013; Kono 2013; Kronish 2014; Lowe 2007; Maasland 2007; MacKenzie 2013; Markle-Reid 2011; Mant 2016; McAlister 2014; McManus 2014; O'Carroll 2011; Pergola 2014; Slark 2013; Wan 2016; Wang 2005; Welin 2010) and six used a cluster design (Brotons 2011; Dregan 2014; Johnston 2010; Lowrie 2010; Ranta 2015; Peng 2014). Visual inspection of funnel plots to detect possible reporting bias suggested no asymmetry. Detailed information on each study is provided in Characteristics of included studies.

\section{Participants}

The trials included a total of 33,840 participants with cerebrovascular disease. The mean or median age of participants ranged from 60 years to 74.3 years. Nine studies included participants with a diagnosis of ischaemic stroke (Allen 2009; Boysen 2009; Chiu 2008; Hedegaard 2014; Johnston 2010; Kim 2013; Kono 2013; Slark 2013; Wan 2016), whereas six studies included participants with either ischaemic or haemorrhagic stroke (MIST 2014; Dregan 2014; Jönsson 2014; Lowe 2007; Lowe 2007; Welin 2010), one focused on lacunar strokes (Pergola 2014) and two did not specify stroke subtype (McManus 2014; Wang 2005). Nineteen trials included a broader range of participants with a diagnosis of either stroke or TIA (Allen 2002; Boter 2004; Damush 2015; Eames 2013; Ellis 2005; Flemming 2013; Hanley 2015; Hornnes 2011; Nailed Stroke 2010; Joubert 2009; Kronish 2014; MacKenzie 2013; McManus 2014; Mant 2016; Markle-Reid 2011; McAlister 2014; O'Carroll 2011; Peng 2014; Ranta 2015). The proportion of TIA participants ranged from 1\% (Eames 2013) to 46\% (Flemming 2013).
Four studies focused only on individuals with minor stroke or TIA (Adie 2010; Chanruengvanich 2006; Kerry 2013; Maasland 2007). Other studies included participants with a history of cardiovascular disease or elevated cardiovascular risk factors, and provided separate unpublished data for stroke and TIA participants (Brotons 2011; Evans 2010; Lowrie 2010).

\section{Location}

Seven included trials were conducted in the USA (Allen 2002; Allen 2009; Damush 2015; Flemming 2013; Johnston 2010; Kronish 2014; Pergola 2014), four in Canada (Evans 2010; McAlister 2014; MacKenzie 2013; Markle-Reid 2011), nine in the UK (Adie 2010; Dregan 2014; Ellis 2005; Hanley 2015; Lowe 2007; Lowrie 2010; Mant 2016; McManus 2014; O'Carroll 2011), 10 in other European countries (Boter 2004; Brotons 2011; Hedegaard 2014; Hornnes 2011; Nailed Stroke 2010; Jönsson 2014; Kerry 2013; Maasland 2007; Slark 2013; Welin 2010), four in Australasia (MIST 2014; Eames 2013; Joubert 2009; Ranta 2015), and seven in Asia (Chanruengvanich 2006; Chiu 2008; Kim 2013; Kono 2013; Peng 2014; Wan 2016; Wang 2005). One study was a multicentre trial conducted in five centres in China and Europe (Boysen 2009).

\section{Setting}

Most studies were set in primary care or community settings (Adie 2010; Allen 2002; Allen 2009; Boter 2004; Boysen 2009; Brotons 2011; Chanruengvanich 2006; Dregan 2014; Evans 2010; Hanley 2015; Hornnes 2011; Nailed Stroke 2010; Kerry 2013; Kim 2013; Kono 2013; Kronish 2014; MacKenzie 2013; Mant 2016; Markle-Reid 2011; McManus 2014; O'Carroll 2011; Pergola 2014; Ranta 2015; Wan 2016; Wang 2005). Seven studies were set in outpatient clinics (Chiu 2008; Damush 2015; Ellis 2005; Flemming 2013; Hedegaard 2014; Jönsson 2014; Welin 2010). One study was incorporated into a TIA service that provided screening and diagnostic work-up in a single day (Maasland 2007). One study was based at a stroke prevention centre (McAlister 2014), and another at a veterans' medical centre (Damush 2015). A further two interventions were performed during hospitalisation for acute stroke (Johnston 2010; Slark 2013). Five studies were initiated in the hospital setting (Eames 2013; Joubert 2009; Lowe 2007) with two subsequently continuing the intervention in the community (Eames 2013; Joubert 2009) and one was undertaken either in a hospital (if the participant was still an inpatient), or in the community if discharged (MIST 2014).

\section{Interventions}

See Characteristics of included studies for details of interventions (components, length, frequency).

\section{Intervention categories}

To facilitate analysis and interpretation of study results, we described interventions according to categories pre-specified in the review protocol (educational and behavioural interventions for patients; educational and behavioural interventions for healthcare providers; organisational interventions as defined according to the taxonomy developed by Wensing 2006). Most interventions were multifaceted and contained components that were associated with more than one category, for example studies included organisational elements with varying amounts of education (directed for patients or healthcare professionals). However, to summarise evidence effectively, we categorised interventions according to their predominant components. For example, if organisational elements were considered to have facilitated or 
permitted the delivery of education (e.g. patient education is often a component of multidisciplinary team services (Wensing 2006)) these were classified as organisational. We decided final category assignments by discussion among review authors to reach consensus.

Sixteen studies included educational or behavioural interventions for participants. Nineteen studies included multidisciplinary team services where patient care was delivered according to protocols for screening, education, and treatment or monitoring. Fourteen studies included educational or behavioural interventions for healthcare providers, which usually involved the provision of guidelines or specification of individual patient targets. Less common intervention elements included revision of professional roles (changes in the tasks carried out by pharmacists), collaboration among multidisciplinary teams, knowledge management systems, and quality management. No studies included financial interventions. Just under half of the studies included multidisciplinary teams where patient care was delivered according to protocols for screening, education, and treatment or monitoring. After review and discussion, we agreed that the interventions were categorised predominately as educational or behavioural interventions for patients and organisational interventions. Predominant intervention categories are highlighted in Table 1.

\section{Educational or behavioural interventions for patients}

Sixteen studies involved educational and behavioural interventions for participants (Adie 2010; Boysen 2009; Chanruengvanich 2006; Chiu 2008; Eames 2013; Kim 2013; Kono 2013; Kronish 2014; Lowe 2007; Maasland 2007; MacKenzie 2013; MIST 2014; O'Carroll 2011; Peng 2014; Slark 2013; Wan 2016). None of the interventions investigated by these studies incorporated organisational elements.

The content of 11 studies was largely focused on modifiable risk factors for stroke (Adie 2010; MIST 2014; Boysen 2009; Chanruengvanich 2006; Chiu 2008; Kim 2013; Kono 2013; Maasland 2007; MacKenzie 2013; O'Carroll 2011; Slark 2013). Five interventions delivered education about secondary stroke prevention as part of broader stroke education programmes (Eames 2013; Kronish 2014; Lowe 2007; Peng 2014; Wan 2016).

\section{Organisational interventions}

We included 26 studies that involved predominantly organisational interventions (Allen 2002; Allen 2009; Boter 2004; Brotons 2011; Damush 2015; Dregan 2014; Ellis 2005; Evans 2010; Flemming 2013; Hanley 2015; Hedegaard 2014; Hornnes 2011; Nailed Stroke 2010; Johnston 2010; Jönsson 2014; Joubert 2009; Kerry 2013; Lowrie 2010; Mant 2016; Markle-Reid 2011; McAlister 2014; McManus 2014; Pergola 2014; Ranta 2015; Wang 2005; Welin 2010). Seven interventions addressed secondary stroke prevention as part of a wider set of study aims encompassing post-stroke rehabilitation (interventions with a broad focus) (Allen 2002; Allen 2009; Boter 2004; Damush 2015; Jönsson 2014; Markle-Reid 2011; Welin 2010). Although these organisational interventions generally provided some patient education about secondary stroke prevention, this appeared to be delivered on only one occasion (Allen 2002; Allen 2009) or on an opportunistic basis (Boter 2004; Welin 2010). Conversely, secondary prevention was the main aim of the remaining 18 organisational interventions (interventions specifically targeting secondary prevention). Nine of these interventions included an element of patient education or behavioural counselling directed towards secondary stroke prevention (Brotons 2011; Ellis 2005; Evans 2010; Flemming 2013; Hornnes 2011; Joubert 2009; Kerry 2013; McAlister 2014; Wang 2005). Three studies did not specify the inclusion of patient education elements but directed secondary prevention education for healthcare professionals (Johnston 2010; Kronish 2014; Lowrie 2010).

\section{Control comparators}

Usual care, described as standard care provided by the managing medical team without any enhancement, was used as the control comparator in 30 studies (Adie 2010; Allen 2002; Allen 2009; Boter 2004; Brotons 2011; Chanruengvanich 2006; Chiu 2008; Eames 2013; Ellis 2005; Flemming 2013; Hanley 2015; Hedegaard 2014; Hornnes 2011; Johnston 2010; Jönsson 2014; Joubert 2009; Kerry 2013; Kim 2013; Kono 2013; Lowrie 2010; MacKenzie 2013; Markle-Reid 2011; McManus 2014; MIST 2014; Nailed Stroke 2010; Peng 2014; Ranta 2015; Slark 2013; Wang 2005; Welin 2010).

Seven studies provided control participants with the same initial information and educational advice as the intervention group, without any individualised advice (Boysen 2009; Damush 2015; Evans 2010; Kronish 2014; Lowe 2007; Maasland 2007; Wan 2016 ).

Dregan 2014 reminded practices in the control group to record all stroke-related consultations and adverse events.

An active control group was used in four studies. Control group participants in O'Carroll 2011 received visits from a research fellow, where a generalised, non medication-related discussion was provided. McAlister 2014 used a nurse-led management control group. Mant 2016 randomised participants into either an intensive blood pressure target $(<130 \mathrm{mmHg}$ or a $10 \mathrm{mmHg}$ reduction if baseline pressure was $<140 \mathrm{~mm} \mathrm{Hg}$ ) (active group) or a standard target ( $<140 \mathrm{mmHg}$ ) (control arm). Pergola 2014 used a similar model whereby patients with recent symptomatic lacunar stroke were randomised to one of two levels of systolic BP (SBP) targets: lower: < $130 \mathrm{mmHg}$ (intervention group), or higher: 130 to 149 $\mathrm{mmHg}$ (control group)

\section{Timing}

We included 24 studies that recruited participants immediately following diagnosis of an acute stoke or TIA. These studies initiated interventions following symptoms of an event (Ranta 2015), before hospital discharge (Eames 2013; Hedegaard 2014; Johnston 2010; Joubert 2009; Lowe 2007; MacKenzie 2013; Maasland 2007; Slark 2013), within one week post-discharge (Allen 2002; Allen 2009; Boter 2004; Wang 2005), within one month post-discharge (Adie 2010; MIST 2014; Nailed Stroke 2010; Wan 2016), within three months post-discharge (Boysen 2009; Chanruengvanich 2006; Ellis 2005; Flemming 2013; Jönsson 2014; O'Carroll 2011; Welin 2010), or within 12 months post-discharge (Damush 2015). Twelve studies recruited participants from primary care, outpatient or community settings, within three months (Hanley 2015; Kono 2013; Peng 2014; Ranta 2015), six months (Pergola 2014), nine months (Kerry 2013), 12 months (Brotons 2011; Kim 2013; McAlister 2014), 18 months (Markle-Reid 2011), up to five years (Kronish 2014) post stroke or TIA diagnosis; or ever had a stroke or TIA (Dregan 2014). One study initiated the intervention when participants had been attending an outpatient clinic for at least 12 months (Chiu 2008). Four studies 
did not specify intervention timing (Evans 2010; Lowrie 2010; Mant 2016; McManus 2014).

Five studies involved interventions that were delivered on a single occasion (Lowe 2007; Maasland 2007; Ranta 2015; Slark 2013) or on two occasions (O'Carroll 2011). The remaining studies implemented interventions over a time frame ranging from three months to 36 months. Most interventions studied by trials had durations of between three months and 12 months.

\section{Outcomes}

Details of outcomes are provided in the Characteristics of included studies table.

\section{Funding sources}

Sources of funding were reported by 38 studies (90\%). Most studies were either funded by charities (45\%) or government sources (24\%). Other funding sources included universities, fellowships, industry, and the NHS. Three studies had multiple funding sources and two did not receive any funding.

\section{Excluded studies}

We excluded eight studies that did not report separately on TIA and stroke participants (Amariles 2012; Goessens 2006; Joshi 2012; Ma 2009; Palanco 2011; Spassova 2016; Strandberg 2006; Vernooij 2012); six with no relevant outcomes (Banet 1997; Bokemark 1996; Gillham 2010; Green 2007; Middleton 2004; Nir 2006); three did not present a stroke service intervention (FIMDM_CVD 2010; Johnston
2000; Ornstein 2004); two were not intended to improve modifiable risk factor control (Harrington 2007; Ross 2007), two contained an exercise training program (Rimmer 2000; UMIN000001865) and one was not a RCT (Sides 2012). We will consider these studies for inclusion in a future update. We have provided a summary in the Characteristics of excluded studies table.

\section{Studies awaiting classification}

There were 13 completed trials for which further study information was unavailable (see Characteristics of studies awaiting classification).

\section{Ongoing studies}

We identified 24 eligible studies: 17 were currently recruiting, 2 were not yet recruiting, 3 were classified as ongoing, 1 was active but not recruiting, and one was unknown (see Characteristics of ongoing studies).

\section{Risk of bias in included studies}

We assessed the risk of bias according to Cochrane's tool for assessing risk of bias. We extracted information about methods of randomisation and allocation concealment, blinding of outcome assessors, incomplete outcome data, selective outcome reporting, and any other potential sources of bias for each included study. We assessed three studies at high risk of bias; the remainder were considered to have a low risk of bias. Detailed assessments of risk of bias for each study is presented in Characteristics of included studies. Summary assessments are shown in Figure 2 and Figure 3.

Figure 2. Risk of bias graph: review authors' judgements about each risk of bias item presented as percentages across all included studies.

Random sequence generation (selection bias)

Allocation concealment (selection bias)

Incomplete outcome data (attrition bias): All outcomes

Selective reporting (reporting bias)

Other bias

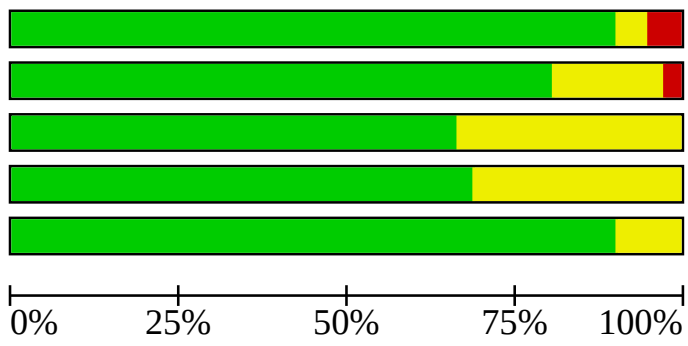

Low risk of bias $\square$ Unclear risk of bias $\quad \square$ High risk of bias


Figure 3. Risk of bias summary: review authors' judgements about each risk of bias item from each study

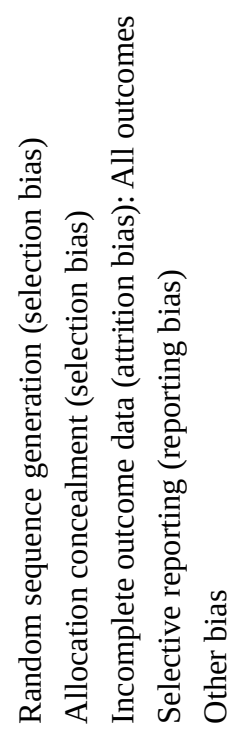

Adie 2010

Allen 2002

Allen 2009

Boter 2004

Boysen 2009

Brotons 2011

Chanruengvanich 2006

Chiu 2008

Damush 2015

Dregan 2014

Eames 2013

Ellis 2005

Evans 2010

Flemming 2013

Hanley 2015

Hedegaard 2014

Hornnes 2011

Johnston 2010

Jönsson 2014

Joubert 2009

Kerry 2013

Kim 2013

Kono 2013

Kronish 2014

Lowe 2007

Lowrie 2010

Maasland 2007

MacKenzie 2013

Mnnt 2016
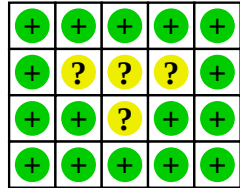
Figure 3. (Continued)

\begin{tabular}{|c|c|c|c|c|c|}
\hline MacKenzie 2013 & + & + & $?$ & + & + \\
\hline Mant 2016 & + & $?$ & + & + & + \\
\hline Markle-Reid 2011 & + & + & + & $?$ & + \\
\hline McAlister 2014 & + & + & + & + & $?$ \\
\hline McManus 2014 & + & + & + & + & + \\
\hline MIST 2014 & + & + & + & + & + \\
\hline Nailed Stroke 2010 & + & + & $?$ & + & $?$ \\
\hline O'Carroll 2011 & + & + & + & + & + \\
\hline Peng 2014 & + & - & $?$ & + & $?$ \\
\hline Pergola 2014 & + & $?$ & $?$ & + & + \\
\hline Ranta 2015 & + & + & $?$ & + & + \\
\hline Slark 2013 & + & + & + & + & + \\
\hline Wan 2016 & + & + & + & + & + \\
\hline Wang 2005 & - & $?$ & $?$ & $?$ & + \\
\hline Welin 2010 & + & + & + & + & + \\
\hline
\end{tabular}

\section{Allocation}

Inclusion criteria for this review required studies to be randomised. All but four studies reported adequate generation of allocation sequence. Two studies were reported as RCTs but did not provide details of randomisation methods (Chanruengvanich 2006; Chiu 2008). Wang 2005 reported that participants were "randomly divided into intervention group (146 cases) and control group (52 cases)". Although the use of randomised methods can be inferred from this statement, the large imbalances in group size were not explained and this included study was considered at high risk of bias. In the study by Jönsson 2014, allocation was undertaken by an administration secretary using lists made by a second study author. Although computer randomisation was used initially, it was deemed that there was high potential for possible bias (Jönsson 2014).

Criteria for adequate allocation concealment were met by all but eight studies. Three trials that did not report randomisation methods also provided insufficient information about allocation concealment (Chanruengvanich 2006; Chiu 2008; Wang 2005). Another five studies with adequate sequence generation contained no information about allocation concealment (Allen 2002; Kim 2013; Mant 2016; Peng 2014; Pergola 2014).

\section{Blinding}

We found that 14 studies reported blinding of outcome assessors for all outcomes (Allen 2009; Boter 2004; Boysen 2009; Chanruengvanich 2006; Eames 2013; Ellis 2005; Hanley 2015; Hedegaard 2014; Hornnes 2011; Kerry 2013; Kronish 2014; MarkleReid 2011; MIST 2014; Wan 2016). A further three studies reported blinding during assessment of selected outcomes (Allen 2002; Johnston 2010; Welin 2010). There were 25 studies for which at least some data were collected by unblinded outcome assessors (Adie 2010; Allen 2002; Brotons 2011; Chiu 2008; Damush 2015; Dregan 2014; Evans 2010; Flemming 2013; Nailed Stroke 2010; Jönsson 2014; Joubert 2009; Kim 2013; Kono 2013; Lowrie 2010; Maasland 2007; MacKenzie 2013; Mant 2016; McAlister 2014; McManus 2014; O'Carroll 2011; Peng 2014; Pergola 2014; Ranta 2015; Slark 2013;
Wang 2005). Following consideration of these 25 studies, we judged that non-blinding of outcome assessors was unlikely to affect the measurement of objective outcomes such as physiological data (e.g. blood pressure), information extracted from medical records, or information measured using validated questionnaires. However, it was unclear whether non-blinding could have affected outcomes obtained from participants via self-reporting (e.g. adherence to medication and self-reported cardiovascular events) (Flemming 2013; Joubert 2009; Kim 2013; Maasland 2007; MacKenzie 2013; MIST 2014; Slark 2013).

\section{Incomplete outcome data}

The proportion of study participants completing follow-up ranged from $70 \%$ (Brotons 2011) to $100 \%$ (Adie 2010; MacKenzie 2013). Two studies did not report the proportion of participants who completed follow-up (Chiu 2008; Wang 2005). In Lowrie 2010, information was only available for those participants with baseline and follow-up data. No missing outcome data were reported for three studies (Adie 2010; MacKenzie 2013; Ranta 2015). We found that 27 studies reported reasons for missing outcome data and we judged these were unlikely to be related to the study outcomes (Boter 2004; Boysen 2009; Brotons 2011; Chanruengvanich 2006; Dregan 2014; Eames 2013; Ellis 2005; Evans 2010; Flemming 2013; Hornnes 2011; Johnston 2010; Kerry 2013; Kim 2013; Kronish 2014; Lowe 2007; Maasland 2007; MacKenzie 2013; Mant 2016; MarkleReid 2011; McAlister 2014; McManus 2014; MIST 2014; O'Carroll 2011; Ranta 2015; Slark 2013; Wan 2016; Welin 2010). The 13 remaining studies did not provide enough information about missing outcome data to permit judgement (Allen 2002; Allen 2009; Chiu 2008; Damush 2015; Hanley 2015; Hedegaard 2014; Nailed Stroke 2010; Joubert 2009; Kono 2013; Lowrie 2010; Peng 2014; Pergola 2014; Wang 2005).

\section{Selective reporting}

Protocols were available for 41 studies, and 31 appeared to be free of selective outcome reporting (Adie 2010; Allen 2009; MIST 2014; Boter 2004; Boysen 2009; Brotons 2011; Chanruengvanich 2006; Dregan 2014; Eames 2013; Evans 2010; Flemming 2013; 
Hanley 2015; Hedegaard 2014; Hornnes 2011; Nailed Stroke 2010; Jönsson 2014; Kerry 2013; Kono 2013; Lowrie 2010; Maasland 2007; MacKenzie 2013; Mant 2016; McAlister 2014; McManus 2014; O'Carroll 2011; Peng 2014; Pergola 2014; Ranta 2015; Slark 2013; Wan 2016; Welin 2010). Johnston 2010 reported primary outcomes as pre-specified, although some secondary outcomes were not reported.

\section{Other potential sources of bias}

It was unclear in some studies if recurrent events were presented as number of events rather than number of people with one or more event (Kono 2013; McAlister 2014; Nailed Stroke 2010; Peng 2014).

\section{Effects of interventions}

See: Summary of findings 1 Educational or behavioural interventions for patients compared to usual care for improving modifiable risk factor control in the secondary prevention of stroke; Summary of findings 2 Organisational interventions compared to usual care for improving modifiable risk factor control in the secondary prevention of stroke

\section{Target achievement of mean reductions, or both}

\section{Blood pressure}

We included 30 studies that reported data on differences in mean systolic or diastolic blood pressure, or both, including where blood pressure target was achieved. Of these, 10 studies evaluated educational or behavioural interventions for participants (Adie 2010; Chanruengvanich 2006; Chiu 2008; Kono 2013; Lowe 2007; Maasland 2007; MacKenzie 2013; MIST 2014; O'Carroll 2011; Slark 2013) and 20 evaluated organisational interventions (Allen 2002; Allen 2009; Brotons 2011; Dregan 2014; Ellis 2005; Evans 2010; Flemming 2013; Hanley 2015; Hornnes 2011; Nailed Stroke 2010; Johnston 2010; Jönsson 2014; Joubert 2009; Kerry 2013; Mant 2016; McAlister 2014; McManus 2014; Pergola 2014; Wang 2005; Welin 2010).

\section{Educational and behavioural interventions for patients}

Pooled data from 11 studies (Adie 2010; Chanruengvanich 2006; Chiu 2008; Kono 2013; Lowe 2007; Maasland 2007; MacKenzie 2013; Mant 2016; MIST 2014; O'Carroll 2011; Slark 2013; N = 1398 ) indicated that educational and behavioural interventions for participants were not associated with significant changes in mean systolic blood pressure (MD $-2.81,95 \% \mathrm{Cl}-7.02$ to 1.39; Analysis 1.1) or mean diastolic blood pressure (MD $-0.83,95 \% \mathrm{Cl}$ -2.80 to 1.13; Analysis 1.2). However, the analyses included one large study that was independently associated with reductions in systolic and diastolic blood pressure (Chiu 2008, N = 160) (Analysis 1.1; Analysis 1.2). Chiu 2008 reported outcome data only for a subgroup of participants with hypertension, so baseline blood pressure levels were higher and therefore easier to improve upon. Kono 2013, a smaller study that involved 70 participants, was associated with a significant reduction in both systolic and diastolic blood pressure within home and clinic readings. The pooled results were associated with a substantial level of statistical heterogeneity $\left(I^{2}=79 \%\right)$. When Chiu 2008 was removed from the analyses, pooled data from the remaining 10 studies did not indicate any intervention effects and statistical heterogeneity was reduced $\left(I^{2}\right.$ $=72 \%$ ). The three studies that reported data on achieving blood pressure targets $(<140 / 90 \mathrm{mmHg}$ or $<130 / 80 \mathrm{mmHg}$ ) indicated that educational and behavioural interventions for patients were not associated with a significant change in the proportion of participants who attained adequate blood pressure control (Adie 2010; Chiu 2008; MacKenzie 2013) (OR 0.74, 95\% Cl 0.39 to 1.44 ; $\mathrm{N}=$ 266; Analysis 1.3; moderate-quality evidence).

\section{Organisational interventions}

Pooled data from 16 studies indicated that organisational interventions were associated with a non-statistically significant reduction in mean systolic blood pressure reduction (MD -1.58, 95\% $\mathrm{Cl}-4.66$ to 1.51; $\mathrm{N}=17,490$; Analysis 2.1) (Brotons 2011; Dregan 2014; Ellis 2005; Evans 2010; Flemming 2013; Hanley 2015; Hornnes 2011; Nailed Stroke 2010; Jönsson 2014; Joubert 2009; Kerry 2013; Mant 2016; McAlister 2014; McManus 2014; Pergola 2014; Welin 2010) (Figure 4; Summary of findings 2). 
Figure 4. Funnel plot of comparison: 2 Organisational interventions versus usual care, outcome: 2.1 Mean systolic blood pressure.

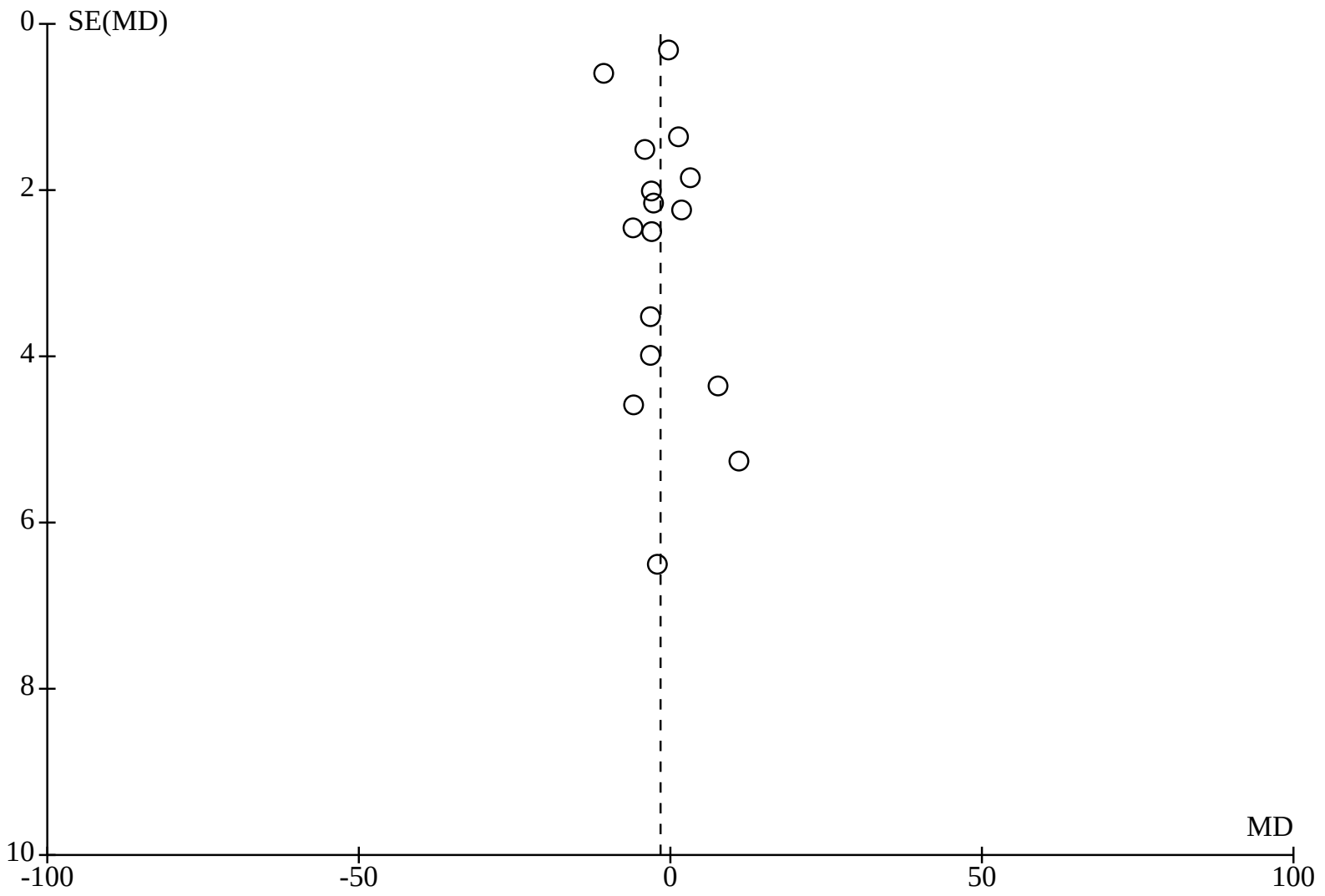

Pooled data from 14 studies indicated that organisational interventions were also associated with a non-statistically significant reduction in mean diastolic blood pressure reduction (MD -0.91, 95\% Cl -2.75 to 0.93; $\mathrm{N}=17,178$; Analysis 2.2) (Brotons
2011; Dregan 2014; Ellis 2005; Evans 2010; Hanley 2015; Hornnes 2011; Nailed Stroke 2010; Jönsson 2014; Joubert 2009; Kerry 2013; Mant 2016; McManus 2014; Pergola 2014; Welin 2010) (Figure 5; Summary of findings 2). 
Figure 5. Funnel plot of comparison: 2 Organisational interventions versus usual care, outcome: 2.2 Mean diastolic blood pressure.

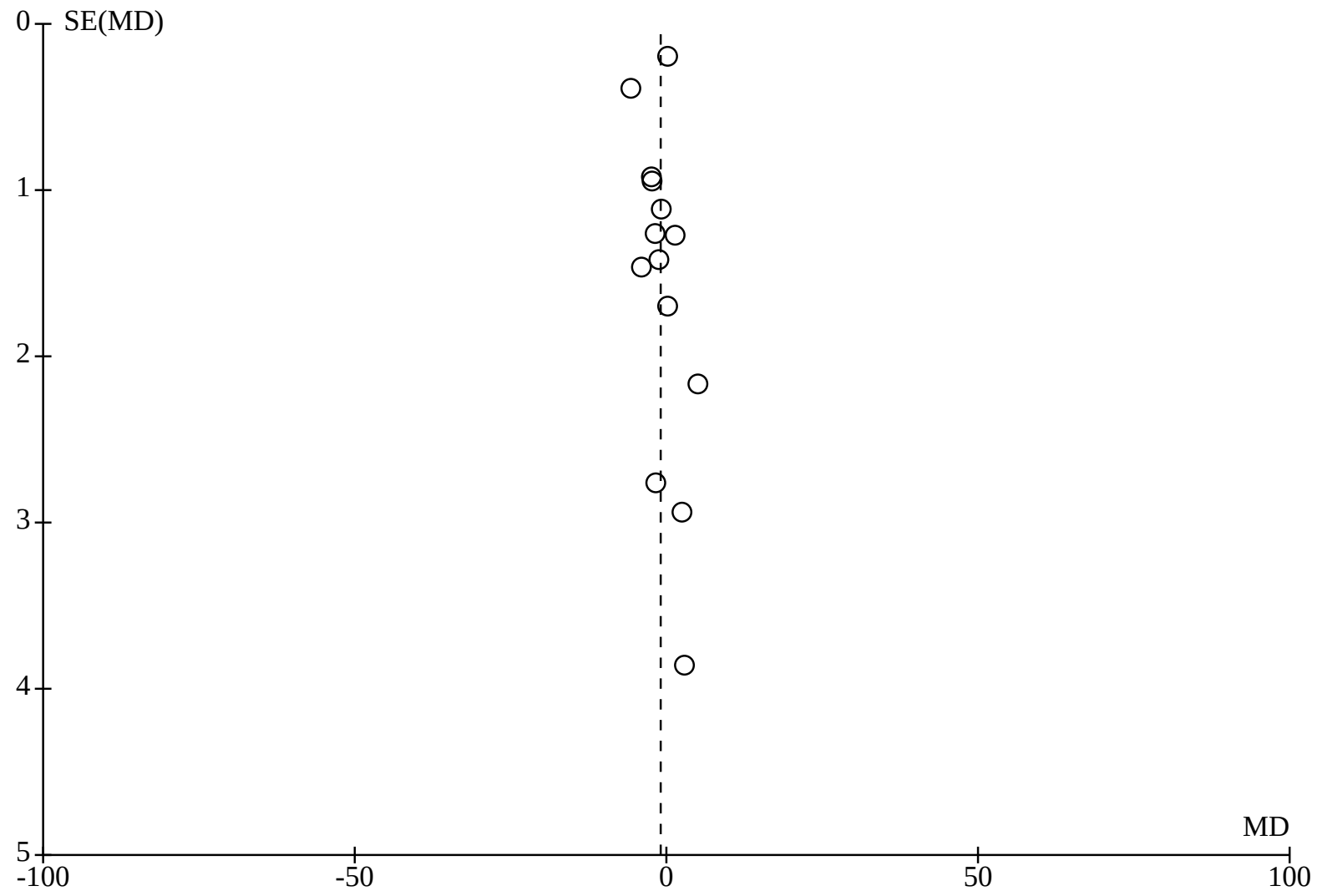

The five studies that were associated with the greatest reductions in mean systolic blood pressure (values ranged from $-3.10 \mathrm{mmHg}$ to $-12.09 \mathrm{mmHg}$ ) combined multidisciplinary team approaches with comprehensive patient education (involving promotion and tracking of adherence to medications and healthy lifestyle behaviours for secondary stroke prevention). These studies focused specifically on secondary stroke prevention and involved regular patient appointments (with a nurse, pharmacist or general practitioner (GP)) and review of multiple stroke risk factors (by a nurse case manager) (Ellis 2005; Flemming 2013; Nailed Stroke 2010; Joubert 2009; Pergola 2014). Nurse case managers informed participants (Ellis 2005; Nailed Stroke 2010) or their GPs (Flemming 2013; Joubert 2009; Pergola 2014) if risk factors deviated from recommended targets (although nurses themselves did not influence medication prescribing).

Consideration of other studies included in the meta-analysis of systolic blood pressure data showed that most interventions were not focused specifically on secondary stroke prevention due to wider study aims (Allen 2002; Welin 2010) or the inclusion of participants with a range of other cardiovascular diseases (Brotons 2011; Evans 2010). Six studies that focused specifically on secondary stroke prevention had a more narrow objective; these largely considered blood pressure control rather than multiple risk factor reduction (Hanley 2015; Hornnes 2011; Kerry 2013; Mant 2016; McManus 2014; Pergola 2014).

Thirteen studies evaluating organisational interventions reported data on achievement of blood pressure targets (Allen 2009; Brotons 2011; Dregan 2014; Flemming 2013; Hanley 2015; Hornnes 2011; Nailed Stroke 2010; Johnston 2010; Jönsson 2014; Joubert 2009; McAlister 2014; Pergola 2014; Wang 2005). Targets varied by study and according to participant co-morbidities; most studies specified a blood pressure target of $\leq 140 / 90 \mathrm{mmHg}$ or $\leq 130 / 80 \mathrm{mmHg}$ for participants with diabetes. Some studies defined alternative blood pressure targets unrelated to co-morbidities of systolic values between $130 \mathrm{mmHg}$ and $140 \mathrm{mmHg}$ and diastolic values of 70 $\mathrm{mmHg}$ to $90 \mathrm{mmHg}$. Pergola 2014 allocated participants to achieve a systolic blood pressure target of either $<130 \mathrm{mmHg}$ or 130 to $149 \mathrm{mmHg}$. Pooled data indicated that organisational interventions were associated with a significant increase in the proportion of participants who attained blood pressure targets (OR $0.70,95 \% \mathrm{Cl}$ 0.53 to $0.92 ; \mathrm{N}=23,631 ; \mathrm{P}=0.01$; Analysis 2.3; Figure 6; Summary of findings 2). Sensitivity analysis was undertaken for target blood pressure. A statistically significant result was observed for all results (Summary of findings 2). 
Figure 6. Funnel plot of comparison: 2 Organisational interventions versus usual care, outcome: 2.3 Blood pressure target achievement.

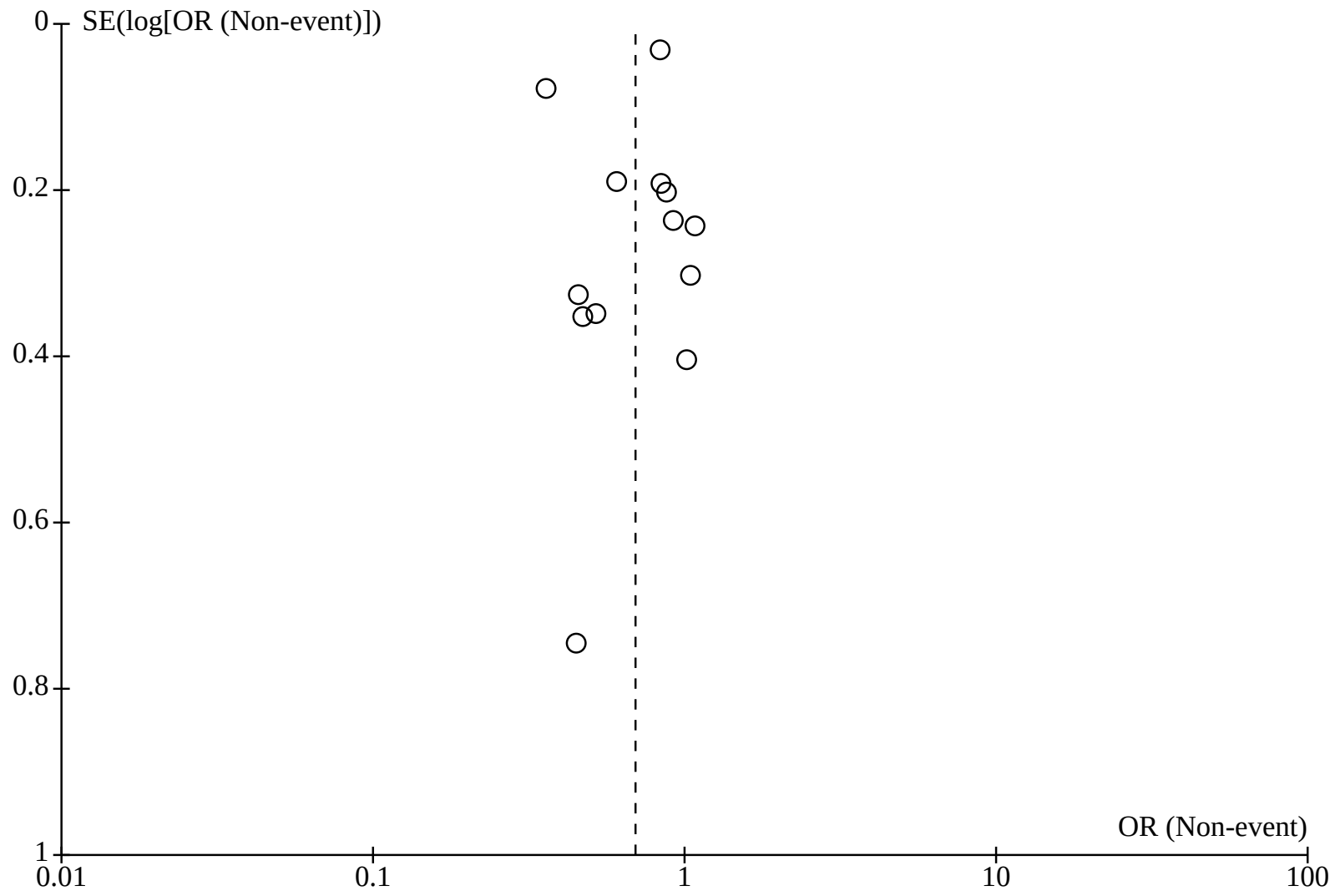

Seven studies reported involving multidisciplinary team members that included nurses, pharmacists able to prescribe, stroke specialist, care co-ordinator, GP, and a neurologist (Allen 2009; Flemming 2013; Hornnes 2011; Nailed Stroke 2010; Jönsson 2014; Joubert 2009; McAlister 2014). Sensitivity analysis of this subgroup revealed a significant effect of involving multidisciplinary team members on target achievement (OR $1.28,95 \% \mathrm{Cl} 1.02$ to $1.62 ; \mathrm{P}=$ 0.04). Heterogeneity was moderate $\left(I^{2}=26 \%\right)$. A further subgroup analysis of nurse led care again identified a significant effect (OR 1.39, 95\% Cl 1.09 to1.78; $\mathrm{P}=0.008$ ) with little difference in heterogeneity $\left(I^{2}=15 \%\right.$ ) (Allen 2002; Flemming 2013; Hornnes 2011; Nailed Stroke 2010; Jönsson 2014; McAlister 2014). McAlister 2014 involved pharmacists who were able to prescribe. This group showed a significant percentage of participants who achieved the targets for blood pressure and LDL cholesterol. Multivariate analyses confirmed there was greater attainment of the guidelinerecommended targets in the pharmacist-led group compared with the nurse-led group (OR 2.12, $95 \% \mathrm{Cl} 1.06$ to $4.23 ; \mathrm{P}=0.03$ ). It is noted that no control group comparison was made.

\section{Total cholesterol}

We included 17 studies that reported cholesterol data, of which seven included educational and behavioural interventions for patients (Adie 2010; Chanruengvanich 2006; Chiu 2008; Kim 2013; Maasland 2007; MIST 2014; Slark 2013) and 10 included predominantly organisational interventions (Allen 2002; Brotons
2011; Dregan 2014; Ellis 2005; Evans 2010; Jönsson 2014; Joubert 2009; Lowrie 2010; McAlister 2014; Wang 2005).

\section{Educational and behavioural interventions for patients}

Pooled data from seven studies indicated that educational and behavioural interventions for patients were not associated with changes in mean total cholesterol levels (MD 0.10, 95\% Cl -0.28 to $0.47 ; \mathrm{N}=721$; Analysis 1.4) (Adie 2010; Chanruengvanich 2006; Chiu 2008; Kim 2013; Maasland 2007; MIST 2014; Slark 2013). Only Adie 2010 reported achievement of total cholesterol targets (total cholesterol $\leq 4 \mathrm{mmol} / \mathrm{L}$ ) and found no significant difference between the intervention and control groups (OR 1.78, 95\% Cl 0.60 to $5.30 ; \mathrm{N}=56$; Analysis 1.5).

\section{Organisational interventions}

Organisational interventions were not associated with changes in mean total cholesterol levels (Brotons 2011; Dregan 2014; Ellis 2005; Evans 2010; Joubert 2009; Lowrie 2010; McAlister 2014) (MD -0.00, $95 \% \mathrm{Cl}-0.04$ to $0.03 ; \mathrm{N}=11,955$; Analysis 2.4 ). Pooled data from six studies indicated that organisational interventions were also associated with changes in the achievement of total cholesterol targets, although the substantial level of statistical heterogeneity observed in this analysis meant that results should be interpreted with caution (OR $0.78,95 \% \mathrm{Cl} 0.53$ to $1.17 ; \mathrm{N}=12,539 ; \mathrm{I}^{2}=80 \%$; Analysis 2.5) (Allen 2009; Dregan 2014; Jönsson 2014; Joubert 2009; Lowrie 2010; Wang 2005). It should be noted that in this metaanalysis we considered the outlying study with the largest effect 
size to be at high risk of bias due to concerns about the adequacy of the randomisation procedures (Wang 2005). Furthermore, the authors of this trial did not specify risk factor targets, stating instead that the results of blood fat tests were either classified as qualified or disqualified. When we removed this study from the meta-analysis, there were no changes in the achievement of total cholesterol targets (varying from $<4.0$ to $<5.0 \mathrm{mmol} / \mathrm{L}$ ) when we pooled the data from the remaining five studies, and statistical heterogeneity was absent $\left(I^{2}=0 \%\right)$.

\section{Low density lipoprotein (LDL)}

We included 11 studies that reported LDL data, of which four evaluated educational and behavioural interventions for patients (Chiu 2008; Kono 2013; Maasland 2007; MIST 2014) and seven evaluated organisational interventions (Brotons 2011; Evans 2010; Flemming 2013; Nailed Stroke 2010; Jönsson 2014; Kronish 2014; McAlister 2014).

\section{Educational and behavioural interventions for patients}

Pooled data from four studies indicated that educational and behavioural interventions for patients were not associated with changes in mean LDL levels (Summary of findings 1) (Chiu 2008; Kono 2013; Maasland 2007; MIST 2014). A low level of statistical heterogeneity was observed (MD $-0.13,95 \% \mathrm{Cl}-0.28$ to $0.02 ; \mathrm{N}=495 ; \mathrm{I}^{2}=12 \%$; Analysis 1.6). Chiu 2008 reported improvements in LDL levels (MD $-0.13 \mathrm{mmol} / \mathrm{L} ; 95 \% \mathrm{Cl}-0.28$ to $0.02 ; P=0.1$ ). Data, however, were only presented for a subgroup of participants with hypercholesterolaemia (i.e. those with the greatest potential for improvement). Maasland 2007 reported significant reductions in LDL for both the intervention and control groups, with no significant differences between the groups. Only Chiu 2008 presented data on the achievement of LDL targets (LDL $2.6 \mathrm{mmol} / \mathrm{L}$ or, if LDL was not available, total cholesterol $<4.1 \mathrm{mmol} /$ L) and no significant improvements were reported (Chiu 2008). Neither of the two other studies identified a significant effect on LDL.

\section{Organisational interventions}

Pooled data from five studies indicated that organisational interventions were associated with a significant reduction in mean LDL levels (Analysis 2.6) (MD $-0.19 \mathrm{mmol} / \mathrm{L}, 95 \% \mathrm{Cl}-0.30$ to -0.09 ; $\mathrm{n}=1154$ ) (Summary of findings 2) (Brotons 2011; Evans 2010; Flemming 2013; Nailed Stroke 2010; McAlister 2014). There was, however, no statistically significant improvement in achieving LDL targets (OR $0.73,95 \% \mathrm{Cl} 0.47$ to $1.13 ; \mathrm{N}=1790 ; \mathrm{P}=0.15$; Analysis 2.7; Summary of findings 2$)$. Heterogeneity was high $\left(I^{2}=75 \%\right)$. Sensitivity analysis of a subgroup of nurse-led care to achieve LDL levels were not associated with achieving LDL targets (OR 0.73, 95\% $\mathrm{Cl} 0.47$ to 1.13; $\mathrm{N}=1790$; Analysis 2.7) (Flemming 2013; Jönsson 2014; Nailed Stroke 2010). One study that involved prescribing pharmacists identified a greater association with achieving LDL target levels (fasting LDL $\leq 2 \mathrm{mmol} / \mathrm{L}$ ) (OR 2.04, $95 \% \mathrm{Cl} 1.26$ to 3.31 ; $\mathrm{P}=0.004)$ than non-prescribing healthcare practitioners. However, no control was compared.

\section{High density lipoprotein (HDL)}

Seven studies reported data on HDL, of which three evaluated an educational or behavioural intervention for patients (Chanruengvanich 2006; Kono 2013; MIST 2014), and four evaluated organisational interventions (Brotons 2011; Evans 2010; Flemming 2013; McAlister 2014). To ensure homogeneous data presentation, we multiplied the mean values by -1 to ensure that all scales pointed in the same direction for both educational and behavioural interventions for patients and for organisations interventions (Analysis 1.7; Analysis 2.8). This is in accordance with guidance from the Cochrane Handbook for Systematic Reviews of Interventions (Higgins 2011c).

\section{Educational and behavioural interventions for patients}

Three studies reported mean HDL levels; no significant intervention effect was observed (Chanruengvanich 2006; Kono 2013; MIST 2014) (MD - $0.03,95 \% \mathrm{Cl}-0.11$ to $0.05 ; \mathrm{N}=452$; Analysis 1.7 ). Kono 2013 reported a significant increase in HDL six months after the intervention (control $=56.3 \mathrm{mg} / \mathrm{dL}$ versus intervention $62.6 \mathrm{mg} / \mathrm{dL}$ ). No studies reported data on HDL target achievement.

\section{Organisational interventions}

We observed no significant intervention effects on mean HDL levels when we pooled data from four studies (Brotons 2011; Evans 2010; Flemming 2013; McAlister 2014) (MD $-0.02,95 \% \mathrm{Cl}-0.09$ to 0.04 ; N = 522; Analysis 2.8). Flemming 2013 reported data on HDL target achievement (fasting HDL $>1.0 \mathrm{mmol} / \mathrm{L}$ in men; $>1.3 \mathrm{mmol} / \mathrm{L}$ in women) and we observed no significant differences between the intervention and control groups (OR $0.79,95 \% \mathrm{Cl} 0.20$ to $3.07 ; \mathrm{N}=$ 36; Analysis 2.9).

\section{Triglycerides}

Seven studies reported data on triglycerides. Three studies involved educational and behavioural interventions for patients (Chiu 2008; Kim 2013; Maasland 2007), and four involved organisational interventions (Brotons 2011; Evans 2010; Flemming 2013; McAlister 2014).

\section{Educational and behavioural interventions for patients}

There were no effects of patient educational and behavioural interventions on mean triglyceride levels (Chanruengvanich 2006; Kim 2013; Maasland 2007) (MD $-0.01,95 \% \mathrm{Cl}-0.31$ to 0.30 ; $\mathrm{N}=$ 182; Analysis 1.8). No studies reported data on triglyceride target achievement.

\section{Organisational interventions}

There were no effects of organisational interventions on mean triglyceride levels (Brotons 2011; Evans 2010; Flemming 2013; McAlister 2014) (MD -0.08, $95 \% \mathrm{Cl}-0.21$ to $0.04 ; \mathrm{N}=485$; Analysis 2.10). Flemming 2013 reported data on the achievement of triglyceride targets (fasting triglycerides $<1.7 \mathrm{mmol} / \mathrm{L}$ ) and no significant differences were observed between the intervention and control groups (OR 4.00, 95\% $\mathrm{Cl} 0.85$ to $18.84 ; \mathrm{N}=36$; Analysis 2.11).

\section{Mean HbA1c}

Eight studies reported data on HbA1c outcomes. Studies were not restricted to participants with diabetes. Two studies evaluated a patient educational or behavioural intervention (Chiu 2008; Kono 2013) and six studies evaluated organisational interventions (Allen 2009; Ellis 2005; Evans 2010; Flemming 2013; Jönsson 2014; Wang 2005).

\section{Educational and behavioural interventions for patient}

Kono 2013 reported mean HbA1c; however, no significant difference was identified between the control and intervention 
groups, despite individual lifestyle education (MD $-0.11,95 \% \mathrm{Cl}$ -0.39 to $0.17 ; \mathrm{N}=70$; Analysis 1.9 ; Summary of findings 1 ). Chiu 2008 reported an outcome relating to $\mathrm{HbAlc}$ target achievement ( $\mathrm{HbA} 1 \mathrm{c}<7 \%$ or fasting blood glucose $<7.0 \mathrm{mmol} / \mathrm{L}$ or random postprandial blood glucose $<11.1 \mathrm{mmol} / \mathrm{L}$ ) and no significant differences between the intervention and control groups were observed (OR 1.53, 95\% Cl 0.57 to 4.08; $\mathrm{N}=67$; Analysis 1.10).

\section{Organisational interventions}

Pooled data from four studies indicated no effects of organisational interventions on mean HbA1c levels (Analysis 2.12) (Ellis 2005; Evans 2010; Flemming 2013; Jönsson 2014). No significant intervention effect was observed and a considerable level of statistical heterogeneity was present $\left(I^{2}=98 \%\right)(O R 0.25,95 \% \mathrm{Cl} 0.02$ to $3.33 ; \mathrm{N}=553$; Analysis 2.13).

\section{Body mass index (BMI)}

Eight studies reported BMI results, of which two evaluated a patient educational or behavioural intervention (Kono 2013; Maasland 2007), and six evaluated organisational interventions (Brotons 2011; Flemming 2013; Jönsson 2014; Joubert 2009; McAlister 2014; Wang 2005).

\section{Educational and behavioural interventions for patients}

Two studies reported data on mean BMI. No significant intervention effects were observed (Kono 2013; Maasland 2007) (MD 0.22, 95\% $\mathrm{Cl}-0.85$ to $1.29 ; \mathrm{N}=127$; Analysis 1.11; Summary of findings 1 ).

\section{Organisational interventions}

Pooled data from five studies indicated no significant effect (Brotons 2011; Flemming 2013; Jönsson 2014; Joubert 2009; McAlister 2014). Heterogeneity was moderate $\left(I^{2}=48 \%\right)$. However, when Jönsson 2014, assessed at high risk of bias, was removed from the analysis, heterogeneity was low $\left(\mathrm{I}^{2}=0\right)$ and there was a statistically significant reduction in mean BMI levels (MD -0.83 $\mathrm{kg} / \mathrm{m}^{2}, 95 \% \mathrm{Cl}-1.47$ to $-0.19 ; \mathrm{P}=0.01$; Analysis 2.14 ; Summary of findings 2).

Two studies measured the achievement of BMI targets (Flemming 2013; Wang 2005) (OR 0.58, 95\% Cl 0.31 to 1.08; $\mathrm{N}=234$; Analysis 2.15). In Wang 2005, the intervention was associated with improvements in BMI target achievement that bordered on statistical significance (OR $1.73,95 \% \mathrm{Cl} 0.93$ to $3.25 ; \mathrm{P}=0.08$ ). However, the study was considered at high risk of bias and the BMI target was not specified. In Flemming 2013, no significant differences in the achievement of the specified BMI target $(<25 \mathrm{~kg} /$ $\mathrm{m}^{2}$ ) were observed between the intervention and control groups.

\section{Cardiovascular risk score}

\section{Organisational interventions}

Flemming 2013 reported data on the Framingham cardiovascular risk scores. The Framingham point score can be used to provide an estimate of an individual's 10-year risk of developing cardiovascular disease (Anderson 1991; Wilson 1998). Flemming 2013 reported that the intervention group demonstrated a significantly greater reduction in Framingham cardiovascular risk score when compared with the control group (MD -6.50; $95 \% \mathrm{Cl}$ -10.22 to $-2.78 ; \mathrm{P}<0.05$; Analysis 2.16), although the available study data were insufficient to discern the magnitude of cardiovascular risk reduction.

\section{Adherence to secondary prevention medications}

We included 21 studies that measured adherence to secondary prevention medications. Of these, 13 involved educational and behavioural interventions for participants (Damush 2015; Dregan 2014; Eames 2013; Hedegaard 2014; Kim 2013; Kronish 2014; Maasland 2007; MacKenzie 2013; MIST 2014; O'Carroll 2011; Peng 2014; Slark 2013; Wan 2016), and eight involved organisational interventions (Allen 2009; Boter 2004; Ellis 2005; Flemming 2013; Hornnes 2011; Johnston 2010; Joubert 2009; McAlister 2014).

\section{Educational and behavioural interventions for patients}

We included 13 studies that reported the effects of patient education on adherence to secondary prevention medications (Damush 2015; Dregan 2014; Eames 2013; Hedegaard 2014; Kim 2013; Kronish 2014; Maasland 2007; MacKenzie 2013; MIST 2014; O'Carroll 2011; Peng 2014; Slark 2013; Wan 2016). Data could not be pooled due to methodological heterogeneity (differences in outcome measurements). Only Eames 2013 reported adequate blinding of outcome assessors. We assessed that non-blinding of outcome assessors may have influenced the data collected by 10 studies that assessed participants' self-reported medication adherence during face-to-face or telephone interviews with outcome assessors (Damush 2015; Hedegaard 2014; Kim 2013; Kronish 2014; Maasland 2007; MacKenzie 2013; MIST 2014; O'Carroll 2011; Peng 2014; Slark 2013). However, non-blinding of outcome assessors was unlikely to affect the adherence outcome data collected by O'Carroll 2011 because data were obtained using a previously validated questionnaire that was administered to participants, and electronic pill containers. Similarly, non-blinding of outcome assessors was unlikely to affect adherence outcome data obtained via a pharmacist review of prescription renewal patterns (MacKenzie 2013), and another study that modified a previously validated questionnaire (Wan 2016). Please see Characteristics of included studies for full evaluations of the risk of bias for the included studies.

Most studies measuring medication adherence outcomes found no significant differences between the intervention and control groups on any indicator of adherence (Summary of findings 1 ). The studies by Damush 2015, Dregan 2014, Eames 2013, Hedegaard 2014, Kim 2013, Kronish 2014, Maasland 2007, MIST 2014, and Slark 2013 found no significant differences between the intervention and control groups in participants' self-reported adherence to secondary prevention medications. MacKenzie 2013 evaluated adherence to antihypertensive medication through participants' self-reported missed medication doses and a pharmacist-led review of participants' prescription renewal patterns. No significant differences in the number of missed pills or prescription renewals were observed between the intervention and control groups.

Three studies reported significant differences in medication adherence between the participants in the intervention and control groups (O'Carroll 2011; Peng 2014; Wan 2016). O'Carroll 2011 conducted a repeated measures analysis of self-reported adherence to antihypertensive medication over a time frame of three months, assessed using the Medication Adherence Report Scale (Horne 2006). Here, O'Carroll 2011 reported that a "significantly greater improvement in the intervention group" with regards to total medication adherence $(P=0.027)$, although the clinical implications of this effect could not be discerned from the available study data. O'Carroll 2011 also evaluated 
antihypertensive medication adherence by obtaining data from electronic pill containers to determine the "percentage of doses taken", "percentage of days on which the correct dose was taken" and "percentage of doses taken on schedule". The trialists reported that "the intervention group had higher adherence on all measures than the control group, although this was only significant for percentage doses taken on schedule $(P=0.048)$ ". More specifically, it was reported that the intervention group took $9.79 \%$ (SD 16.59) more doses on schedule when compared with the control group (O'Carroll 2011).

Peng 2014 reported a significant difference in adherence to statin use between the participants in the intervention and control groups at 12 months, measured by review of medical records. Peng 2014 conducted a trial using the SMART structured program, which compared usual care with a guideline-recommended medication regimen with algorithmic lifestyle modification, in addition to online accessible educational material. It was reported that the SMART group achieved $56 \%$ adherence compared to $33 \%(P=0.006)$ in the usual treatment group. However, there were no significant differences reported in the adherence of other measures between the groups: antiplatelet drug use, antihypertensive drug use and antidiabetic drug use.

Wan 2016 also reported a significantly higher medication adherence which was adjusted over time within the intervention. In this study, stroke nurses engaged participants in self-identified goal setting, encouraged via telephone follow-up. Wan 2016 reported 92.3\% adherence at three-months follow-up, increasing to $96 \%$ adherence at six months, compared to $89 \%$ and $87 \%$ at three and six months respectively $(P<0.001)$.

\section{Organisational interventions}

Four studies reported data on the proportion of participants who were compliant with warfarin therapy (Johnston 2010; Joubert 2009), anticoagulants (Allen 2009), or antithrombotic medication (Flemming 2013). Three studies measured compliance with antihypertensive medication (Hornnes 2011; Johnston 2010; McAlister 2014) and three measured compliance with statin medication (Flemming 2013; Johnston 2010; McAlister 2014). Two further studies reported the proportion of participants using secondary prevention medications as prescribed (Boter 2004; Ellis 2005). Medication compliance was either measured through participant self-report (Allen 2009; Boter 2004; Ellis 2005; Flemming 2013; Hornnes 2011; Joubert 2009) or an analysis of filled prescription data and International Normalised Ratio (INR) blood test records (Johnston 2010; McAlister 2014). Five of the six studies reported blinding of outcome assessors when collecting data on medication compliance (Boter 2004; Ellis 2005; Hornnes 2011; Johnston 2010; McAlister 2014), whereas Joubert 2009 did not provide any information regarding this outcome. Data were not pooled because there was substantial heterogeneity in the methods used to obtain outcome data.

Where results were provided for self-reported medication adherence, no difference was seen between the control and intervention groups in four studies (Allen 2009; Boter 2004; Flemming 2013; Johnston 2010). Hornnes 2011 noted an improvement in antihypertension compliance without an improvement in consequent blood pressure.
McAlister 2014 identified that most participants were documented to be receiving secondary prevention medication at baseline. However, none met guideline targets for parameters such as blood pressure. In this study, a nurse led one intervention group and a pharmacist led a second. It was noted that there was a significant improvement in medication compliance between the intervention groups with improvements in blood pressure and LDL levels at six months.

\section{Secondary outcomes}

\section{Secondary stroke}

\section{Educational and behavioural interventions for patients}

Four studies reported data on the proportion of participants who experienced a recurrent stroke or TIA (Kono 2013; MacKenzie 2013; MIST 2014; Peng 2014) (OR 0.82, 95\% Cl 0.37 to 1.84; N = 4333; Analysis 1.12). Blinding of outcome assessors was not reported in any study. Mackenzie 2013 observed no significant difference in the number of recurrent strokes (assessed from clinical record review) between the intervention and control groups. Both MIST 2014 and Peng 2014 observed no significant difference in the number of strokes or TIAs at 12 months between the intervention and control groups (OR 1.09, 95\% Cl 0.52 to 2.30; $\mathrm{N}=4207$; Analysis 1.13). Kono 2013 reported a reduction in further strokes or TIAs when a multifaceted approach was taken in secondary prevention (OR 0.08; $95 \% \mathrm{Cl} 0.00$ to $1.47 ; \mathrm{P}=0.09$ ). This approach provided education on exercise, salt intake, and addressed blood pressure. It is noted that the sample size was small and based at a single hospital.

\section{Organisational interventions}

Four studies recorded the proportion of participants who experienced at least one recurrent stroke or TIA (Allen 2002; Kerry 2013; Wang 2005; Welin 2010) (OR $0.66,95 \%$ Cl 0.23 to 1.86 ; N = 791; Analysis 2.17). Results were presented as the percentage of participants who had experienced a secondary stroke. In three studies, data on the incidence of recurrent stroke were obtained by blinded outcome assessors from clinical record review (Allen 2002; Welin 2010) or administration of patient questionnaires (Kerry 2013). Wang 2005 did not specify the method used to determine recurrent stroke events and no blinding of outcome assessors was reported. Pooled data from all four studies suggested that organisational interventions were not associated with changes in the proportion of participants who experienced at least one recurrent stroke (OR $0.66,95 \% \mathrm{Cl} 0.23$ to $1.86 ; \mathrm{N}=791$; Analysis 2.17). However, the analysis was associated with substantial statistical heterogeneity $\left(1^{2}=77 \%\right)$ due to an outlying study that was assessed at high risk of bias (Wang 2005). When Wang 2005 was removed from the analysis no intervention effect was observed among the three remaining studies.

Five studies provided data on the number of participants with secondary strokes or TIAs that occurred during follow-up (measured at end of study per protocol) (Boysen 2009; Ellis 2005; Hornnes 2011; Markle-Reid 2011; Ranta 2015). Data on secondary stroke events were obtained by blinded outcome assessors following a review of clinical records (Boysen 2009; Hornnes 2011) or face-to-face interviews with study participants (Markle-Reid 2011). Ranta 2015 observed vascular events (either stroke or stroke and TIA) at 90 days and observed a non-significant reduction in participants with one or more events (Analysis 2.18; Analysis 2.20). Results were presented as the number or percentage 
of participants who had experienced a secondary stroke, except for Ellis 2005 where it was unclear whether the results were for individual participants or total event numbers.

\section{Secondary cardiovascular events}

We included 16 studies that reported data on secondary vascular events. Of these, four studies evaluated educational and behavioural interventions for patients (Kono 2013; MacKenzie 2013; MIST 2014; Peng 2014) and 12 evaluated organisational interventions (Allen 2002; Boysen 2009; Brotons 2011; Ellis 2005; Flemming 2013; Hornnes 2011; Kerry 2013; Markle-Reid 2011; McAlister 2014; Ranta 2015; Wang 2005; Welin 2010).

\section{Educational and behavioural interventions}

Three studies reported data on the proportion of participants who experienced a secondary cardiovascular event during followup (Kono 2013; MIST 2014; Peng 2014). These were presented as the percentage of participants who had experienced a secondary stroke. Kono 2013 reported a significantly lower number of people with cardiovascular events in the intervention group compared with the control at the end of the study (OR $0.12,95 \% \mathrm{Cl} 0.01$ to 1.01; $P=0.05$ ). MIST 2014 and Peng 2014 observed no significant difference in the number of people with cardiovascular events at 12 months between the intervention and control groups (OR 0.82, 95\% $\mathrm{Cl} 0.28$ to $2.37 ; \mathrm{P}=0.71$ ).

\section{Organisational interventions}

Brotons 2011 reported data on the proportion of participants who experienced a secondary cardiovascular event during followup. The data were collected by non-blinded outcome assessors following a review of clinical records and interviews with study participants. No significant intervention effect was observed (OR $1.48,95 \% \mathrm{Cl} 0.79$ to $2.77 ; \mathrm{N}=324$; Analysis 2.21 ).

Ellis 2005 and McAlister 2014 reported data on the number of people with secondary cardiovascular events that occurred before the end of the study. A non-significant improvement was observed (OR $1.48,95 \% \mathrm{Cl} 0.79$ to 2.77 ; $\mathrm{P}=0.56$ ).

\section{Myocardial infarction and ischaemic heart disease}

\section{Educational and behavioural interventions}

Three studies reported the number of myocardial infarctions that occurred after educational and behavioural interventions in individual participants (Kono 2013; MIST 2014; Peng 2014). Two studies did not observe an improvement in the number of cardiovascular deaths (MIST 2014; Peng 2014). Kono 2013 observed significantly less rates of myocardial infarction and angina after a median follow-up period of 2.9 years (OR $0.53,95 \% \mathrm{Cl} 0.17$ to 1.65 ; Analysis 1.14).

\section{Organisational interventions}

Ellis 2005 observed no significant differences in the number of ischaemic heart disease events after a mean follow-up duration of 3.6 years (MD $-0.91,95 \% \mathrm{Cl}-2.75$ to $0.93 ; \mathrm{N}=17,178$; Analysis 2.22). Two studies reported the number or percentage of myocardial infarctions that occurred during follow-up (Boysen 2009; McAlister 2014) and no significant intervention effect was seen (Analysis 2.22; Analysis 2.23). Data were collected by blinded outcome assessors in both studies following clinical record review (Boysen 2009; McAlister 2014) or interviews with study participants (Ellis 2005).

\section{Vascular death}

\section{Educational and behavioural interventions}

MIST 2014 reported data on the number of cardiovascular deaths. No improvement was observed (OR $1.34,95 \% \mathrm{Cl} 0.30$ to $6.07 ; \mathrm{N}=$ 386; Analysis 1.15).

\section{Organisational interventions}

Boysen 2009 and Ranta 2015 reported data on vascular deaths. Boysen 2009 reported data on vascular death obtained by blinded outcome assessors following clinical record review. Boysen 2009 observed no significant differences in the number of vascular deaths occurring in the intervention and control groups (OR 0.38 , $95 \% \mathrm{Cl} 0.15$ to $0.97 ; \mathrm{N}=605$; Analysis 2.24). Ranta 2015 reported single blinded data which identified a significant effect on vascular deaths (OR $0.27,95 \% \mathrm{Cl} 0.1$ to $0.73 ; \mathrm{P}=0.01$ ). When these studies were combined the difference remained significant but both had small numbers of events so no firm conclusions could be drawn.

\section{DISCUSSION}

\section{Summary of main results}

This review produced mixed findings regarding the effectiveness of stroke service interventions for the secondary prevention of stroke. We performed meta-analyses where appropriate for the outcomes of blood pressure, lipid profile, HbAlc, body mass index (BMI) and recurrent cardiovascular events. We carried out a qualitative analysis for medication adherence outcomes.

We assessed the quality of the evidence in this review using GRADEpro software and have presented this information in 'Summary of findings' tables. Overall, the evidence for educational or behavioural interventions for patients compared to usual care for improving modifiable risk factor control in the secondary prevention of stroke ranged from low to moderate. The evidence for organisational interventions compared to usual care for improving modifiable risk factor control in the secondary prevention of stroke also ranged from low to moderate. We downgraded evidence due to the small number of studies included and hence wide confidence intervals.

Pooled data for educational and behavioural interventions for participants were not associated with clear differences in any of the review outcomes. Some improvement was observed for medication adherence. O'Carroll 2011 demonstrated significant differences between the intervention and control groups in adherence to secondary prevention medications. MIST 2014 improved self-reported medication adherence using motivational interviewing. Furthermore, Peng 2014 used structured guidelines to statistically improve statin adherence. However, the same treatment protocol did not evoke a similar response in antihypertensive or antiplatelet medication. Interestingly, Kono 2013 developed an intensive lifestyle modification program delivered by healthcare professionals and physical therapists. Kono 2013 documented a statistically significant reduction in blood pressure, $\mathrm{HDL}$ and salt intake, and an increase in physical activity. It must be noted this was a small study of 70 participants. It was identified that the pharmacist education program evaluated by Chiu 2008 was associated with significant improvements in mean systolic blood pressure, mean diastolic blood pressure, and mean LDL levels. However, Chiu 2008 only presented data for a subgroup 
of participants with hypertension or hypercholesterolaemia who, therefore, had the greatest potential for improvement. It may be that educational interventions are more effective for participants with uncontrolled risk factors, and these participants could be targeted in future studies.

The estimated effects of organisational interventions included statistically significant trends towards improving blood pressure target achievement (OR $1.44,95 \% \mathrm{Cl} 1.09$ to $1.90 ; \mathrm{P}=0.01$ ) but not in mean blood pressure (systolic: $\mathrm{MD}-1.58 \mathrm{mmHg}, 95 \% \mathrm{Cl}-4.66$ to 1.51; $\mathrm{P}=0.32$, diastolic: $\mathrm{MD}-0.91 \mathrm{mmHg}, 95 \% \mathrm{Cl}-2.75$ to $0.93 ; \mathrm{P}=$ $0.33)$.

In the meta-analysis of systolic blood pressure data presented in this review, the largest blood pressure reductions were associated with five interventions, all of which included integrated care with input from multidisciplinary teams and provision of comprehensive patient education. This involved promotion and tracking of behaviours for secondary stroke prevention.

During this review, it was noted that multidisciplinary team members were usually involved when an intervention was associated with an improved outcome on secondary prevention. This often included an element of patient education and regular monitoring. For example, a nurse-led educational intervention or a pharmacist checking compliance of prescribed medications (Flemming 2013; Hedegaard 2014). There are many reported benefits of working within an effective multidisciplinary team, who individually bring a variation in knowledge, specialisation and experience, consider different elements of patient care and collectively considers the 'whole' patient (Health Foundation 2014; Institute of Public Care 2013; Lemieux-Charles 2006). These include more patient-centred decision making (Emberson 2003; Rose 1981) and more effective use of resources including increased awareness of resources available (British Cardiac Society 1998; Cappuccio 2002; Rice 2017). It is proposed that patient participation and adherence to educational information and medication could be improved through reinforcement of information by different team members, with varying levels of clinical expertise (Health Foundation 2014; Lemieux-Charles 2006; Swientozielskyj 2015). Some team members may have more time to consider and address any specific patient-related issues (Swientozielskyj 2015). Recognition for continued learning to increase knowledge and skills is more evident within multidisciplinary teams, through shared learning opportunities and experience (Lindson-Hawley 2015). Furthermore, the cohesion and support of the team may lead to greater team member satisfaction, clearer leadership and accountability, and greater inter-professional collaboration (Beswick 1996; Dawber 1951; Lemieux-Charles 2006). It is expected that a proactive team who are motivated to help and support a patient and provide focused patient-centred care will provide this high level of patient support to enable a beneficial outcome on secondary stroke prevention (Health Foundation 2014; Swientozielskyj 2015).

\section{Overall completeness and applicability of evidence}

A limitation of the included studies was the lack of consistently used outcome measures. For example, some studies measured mean blood pressure whereas some measured target achievement with a variety of acceptable ranges. A similar discrepancy was also seen for weight, weight reduction, BMI and percentage body weight. Combining all results in meta-analyses was therefore problematic.
A second limitation was related to variations in study follow-up duration. This review pooled data collected at the end of the study per protocol. However, follow-up duration varied from three to 43 months. The results should therefore be interpreted with some caution since shorter studies may not provide enough time for the interventions to produce an impact on modifiable risk factors. Conversely, medication adherence or compliance would be expected to be better over shorter durations.

\section{Quality of the evidence}

We analysed data from 42 trials involving 33,840 participants with stroke or TIA. Studies were published between 2002 and 2016. The review authors were not blinded to study details (e.g. study authors, journal and results) when assessing the methods. We assessed the quality of each RCT according to Cochrane's tool for assessing risk of bias. We excluded blinding of participants and healthcare providers from assessment because these criteria were unlikely to be met given the nature of the interventions under consideration. We assessed the risk of bias across six domains including sequence generation, allocation concealment, blinding of outcome assessment, incomplete outcome data, selective outcome reporting, and other sources of bias.

Protocols were available for 41 studies and the analysis was described in 28 studies. Wang 2005 did not report randomisation methods (but had unequal group sizes) and Jönsson 2014 used a randomisation method which the review authors felt may introduce bias. Two further studies discussed randomly allocating participants; however, the full method was not available (Chanruengvanich 2006; Chiu 2008). These areas of potential bias raised questions about the validity of these findings.

We assessed the quality of the evidence in this review using GRADEpro GDT software and have presented this information in 'Summary of findings' tables. Overall, the evidence for educational or behavioural interventions for patients compared to usual care for improving modifiable risk factor control in the secondary prevention of stroke ranged from low to moderate (Summary of findings 1). The evidence for organisational interventions compared to usual care for improving modifiable risk factor control in the secondary prevention of stroke also ranged from low to moderate (Summary of findings 2). We downgraded evidence due to the small number of studies included and hence wide confidence intervals.

\section{Potential biases in the review process}

We attempted to identify all RCTs of potential relevance to the review. In addition to a comprehensive search strategy, we attempted to contact the authors of all included trials to identify further published, unpublished and ongoing studies. Visual inspection of funnel plots did not raise any concerns regarding publication bias. We included all eligible RCTs regardless of publication language; we arranged for translation of one study not published in English. It is acknowledged that for secondary events, study authors did not always clarify whether single events in an individual rather than the total number of events over the total number of participants were reported, leading to overestimation of differences between groups. 


\section{Agreements and disagreements with other studies or reviews}

Buckley 2010 conducted a systematic review of the effects of service organisation interventions for the secondary prevention of ischaemic heart disease. Only interventions delivered in primary care were included. The review found that interventions involving certain elements (regular planned patient appointments, patient education and monitoring of medication and risk factors) may be associated with improved control of total cholesterol and blood pressure levels. However, the authors recommended that results should be interpreted with caution due to significant clinical and statistical heterogeneity.

In contrast to Buckley 2010, this systematic review included interventions that were not delivered in primary care and therefore different types of interventions were included (e.g. implementation of discharge orders). The conclusions of this review, however, are in accordance with Buckley 2010 since organisational interventions, including elements of a multidisciplinary team approach and patient education, were associated with the greatest improvements in blood pressure control.

The possible effects of multidisciplinary team services in this review are also supported by the findings of another review of organisational interventions. Wensing 2006 reported that "integrated care services are particularly promising" when considering strategies to improve patient care. This is attributed to the typical multifaceted nature of these interventions. The authors suggested that the incorporation of numerous intervention components may "address a wide range of potential barriers for change". They also stated that "further work should focus on analysing the contributions of the specific components in integrated care services, to identify which particularly contribute to their effectiveness" (Wensing 2006).

\section{AUTHORS' CONCLUSIONS}

\section{Implications for practice}

This review highlighted possible benefits of organisational interventions on the achievement of blood pressure targets. However, we found no clear evidence that organisational interventions can improve other modifiable risk factors (lipid profile, HbA1c, weight, medication adherence) or reduce the incidence of recurrent cardiovascular events. Results also suggest that interventions including patient education alone are unlikely to lead to improvements in modifiable risk factor control or the prevention of recurrent cardiovascular events.

\section{Implications for research}

Future research should focus on the development of more effective interventions to translate secondary prevention recommendations into practice. The findings from this review suggest that educational and behavioural interventions for patients delivered in the absence of organisational change may not be an effective means of achieving this aim. Future research should evaluate the effects of specific components of organisational interventions, including the characteristics of an effective multidisciplinary team. We identified 24 ongoing studies and 11 studies that are awaiting assessment, so a future review update may lead to more robust conclusions.

The stroke service interventions included in this review were found to differ considerably in terms of aims (e.g. degree of focus on secondary stroke prevention), duration, components and mode of delivery. Pre-determined strategies for categorising interventions and their intensity may facilitate the synthesis of future research findings.

\section{ACKNOWLEDGEMENTS}

We thank all of the authors who kindly provided us with additional data about their studies. We are grateful to Joshua Chenyne, Brenda Thomas, Janette Camosso-Stefinovic and Dr Brian Buckley for their contributions to the search strategies. We developed the search strategy with the help of Brenda Thomas (former Cochrane Stroke Group Trials Search Co-ordinator), Joshua Cheyne (Cochrane Stroke Group Information Specialist), and Dr Brian Buckley of the National University of Ireland (Buckley 2010). We adapted the MEDLINE search strategy (Appendix 2) to search other databases.. We are also grateful for the important contributions made by Phi-Anh Tonnu, Chokanan Thaitirarot to study selection and data collection, Dr Victoria Haunton and Dr Aung K Sett who selected studies according to the review criteria, and contributed to data extraction for the previous review. 


\section{R E F E R E N C E S}

\section{References to studies included in this review}

Adie 2010 \{published and unpublished data\}

* Adie K, James MA. Does telephone follow-up improve blood pressure after minor stroke or TIA? Age and Ageing 2010;39:598-603.

Adie K, James MA. Does telephone follow up improve blood pressure after stroke/TIA? Hypertension 2008;52(4):760.

Adie K, James MA. Does telephone follow up improve blood pressure in patients after stroke or TIA? In: Communications to the Autumn Meeting of the British Geriatrics Society, British Geratrics Society, 2008 November 12-14, Birmingham, UK. Harrogate, UK: British Geriatrics Society UK, 2008:64.

\section{Allen 2002 \{published data only\}}

* Allen KR, Hazelett S, Jarjoura D, Wickstrom GC, Hue K, Weinhardt J, et al. Effectiveness of a postdischarge care management model for stroke and transient ischaemic attack: a randomised trial. Journal of Stroke and Cerebrovascular Disease 2002;11(2):88-98.

Allen KR, Hazelett SE, Jarjoura D, Wickstrom G, Hua K, Weinhardt JA, et al. A post-discharge care management model for stroke and transient ischaemic attack: a randomised controlled trial. Stroke 2002;33(1):417 (Abstract P306).

NCT00328471. A post discharge intervention to improve stroke outcomes. clinicaltrials.gov/ct2/show/NCT00328471 (first received 22 May 2006).

Wright K, Allen K, Weinhardt J, Gareri M, Hua K, Hazelett S. Effectiveness of interdisciplinary post-stroke case management in improving patient outcomes: a pilot study. Journal of Stroke and Cerebrovascular Diseases 2000;9(4):205.

Wright K, Hazelett S, Weinhardt J, Jarjoura D, Hua K, Gareri M, et al. The role of the advanced practice nurse in post-stroke care management. Journal of Stroke and Cerebrovascular Diseases 2003;12(5):249 (Abstract 12).

\section{Allen 2009 \{published data only\}}

* Allen K, Hazelett S, Jarjoura D, Hua K, Wright K, Weinhardt J, et al. A randomised trial testing the superiority of a postdischarge care management model for stroke survivors. Journal of Stroke and Cerebrovascular Disease 2009;18(6):443-52.

Allen K, Hazelett S, Jarjoura D, Wright K, Clough L, Weinhardt J. Improving stroke outcomes: implementation of a postdischarge care management model. Journal of Clinical Outcomes Management 2004;11(11):707-14.

Allen K, Hazelett S, Jarjoura D, Wright K, Weinhardt J. Randomized controlled trial of a post-stroke post-discharge care management intervention. Stroke 2008;39(2):531 (Abstract 14).

Allen K, Jarjoura D, Hazelett S, Wickstrom G, Wright K, Hua K. Effectiveness of care management for secondary prevention with TIA/non-disabled stroke survivors. Journal of the American Geriatrics Society 2002;50(4):483:S168.

Allen KR, Hazelett SE, Palmer RP, Jarjoura DG, Wickstrom GC, Weinhardt JA, et al. Developing a stroke unit using the acute care for elders intervention and model of care. Journal of the American Geriatrics Society 2003;51(11):1660-7.

NCT00328471. A post discharge intervention to improve stroke outcomes. clinicaltrials.gov/show/NCT00328471 (first received 22 May 2006).

Boter 2004 \{published and unpublished data\}

Albrecht KW, Algra A, Boter H, Carpay HA, van Gijn J, de Haan R, et al. HESTIA: a randomised clinical trial of a nursing care program for recently discharged stroke patients. In: The 27th International Stroke Conference San Antonio, Texas, February 7-9, 2002 in Journal of Neuro-Ophthalmology: 2002;22(2)129-133. San Antonio (TX): American Stroke Association, 2002:Abstract CTP365.

Albrecht KWJ. Home evaluation of stroke induced aid (HESTIA). Stroke 2000;31(1):2538-9.

Boter H, Rinkel GJE, de Haan R, HESTIA Study Group. A multicentre, randomised controlled trial of an outreach nursing care programme for recently discharged stroke patients. Journal of Neurology 2003;250 Suppl 2:(Abstract 77).

Boter H, Rinkel JE, Haan R. Outreach nurse support after stroke: a descriptive study on patients' and carers' needs, and applied nursing interventions. Clinical Rehabilitation 2004;18(2):156-63.

Boter H, van Delden JJM, de Haan RJ, Rinkel GJE, Home Evaluation of Stroke Induced Aid Study Group. Patients' evaluation of informed consent to postponed information: cohort study. BMJ 2004;329(7457):86-7.

Boter H, van Delden JJM, de Haan RJ, Rinkel GJE. A modified informed-consent procedure in which the complete information is given retrospectively: no objection from participating patients. Nederlands Tijdschrift voor Geneeskunde 2005;149(1):29-32.

Boter H, van Delden JM, de Haan RJ, Rinkel GJE, Home Evaluation of Stroke Induced Aid Study Group. Modified informed consent procedure: consent to postponed information. BMJ 2003;327(7409):284-6.

* Boter H, for the HESTIA Study Group. Multicenter randomised controlled trial of an outreach nursing support program for recently discharged stroke patients. Stroke 2004;35(12):2867-72.

Boter H. Effectiveness of outreach stroke care. Utrecht: University of Utrecht, 2003.

HESTIA Study Group. Home evaluation of stroke induced aid (HESTIA). Stroke 2002;33(6):1732.

Boysen 2009 \{published and unpublished data\}

* Boysen G, Krarup L, Zeng X, Oskedra A, Kõrv J, Andersen G, et al. ExStroke pilot trial of the effect of repeated instructions to 
improve physical activity after ischaemic stroke: a multinational randomised controlled trial. BMJ 2009;339:b2810.

Boysen G, Krarup LH, Zeng X, Oskedra A, Korv J, Andersen G, et al. Failure to promote physical activity after ischaemic stroke. The ExStroke pilot trial. International Journal of Stroke 2008;3(Suppl 1):72 (Abstract FC11-04).

Boysen G, Pedersen A, Meden P, Hansen L, Lindahl M, Zeng X, et al. Physical exercise after acute ischaemic stroke. Exstroke pilot trial. In: 29th International Stroke Conference; 2004 Feb 5 to 7; San Diego, California, USA found in Journal of NeuroOphthalmology 2004;24(2):175-7. San Diego: American Stroke Association, 2004.

Boysen G, Truelsen T, Pedersen A, Hansen L, Lindahl M, Zeng X. Physical activity and the risk of ischaemic stroke. European Journal of Neurology 2005;12(Suppl 2):311.

Krarup L, Gluud C, Truelsen T, Pedersen A, Lindahl M, Hansen L, et al. The ExStroke pilot trial: rationale, design, and baseline data of a randomized multicenter trial comparing physical training versus usual care after an ischaemic stroke. Contemporary Clinical Trials 2007;29(3):410-7.

Krarup LH, Truelsen T, Boysen G. Repeated encouragement to be physically active improves insulin sensitivity after ischaemic stroke. Cerebrovascular Diseases 2008;25(Suppl 2):7 (Abstract 2)

Krarup LH, Truelsen T, Gludd C, Andersen G, Zeng X, Oskedra A, et al. Prestroke physical activity is associated with severity and long-term outcome from first-ever stroke. Neurology 2008;71(17):1313-8.

Krarup LH, Lindhal M, Truelsen T, Gludd C, Boysen G. The risk of falling after stroke is associated with physical inactivity. Cerebrovascular Diseases 2010;29(1):72-73.

NCT00132483. ExStroke pilot trial: physical exercise after acute ischaemic stroke. www.clinicaltrials.gov/ct2/show/ NCT00132483 (first received 22 August 2005).

Truelsen T, Pedersen A, Schnohr P, Bousen G. The ExStroke Trial. Physical activity before ischaemic stroke. Cerebrovascular Diseases 2004;17(Suppl 5):30 (Abstract 5).

Truelsen T, Pedersen A, Schnohr P, Boysen G. The EXSTROKE trial. Physical activity before ischaemic stroke. European Journal of Neurology 2004;11(Suppl 2):16 (Abstract SC124).

Truelsen T, Pedersen A, Schnor P, Boysen G. The EXSTROKE trial. Physical activity before ischaemic stroke. Stroke 2004;35(6):e239 (Abstract 673).

\section{Brotons 2011 \{published and unpublished data\}}

Brotons C, Arino D, Borrás I, Buitrago F, González ML, Kloppe P, et al. Evaluation of the efficacy of a comprehensive programme of secondary prevention of cardiovascular disease in primary care: the PREseAP Study. Atencion Primaria 2006;37(5):295-8.

* Brotons C, Soriano N, Moral I, Rodrigo MP, Kloppe P, Rodríguez AL, et al. Randomized clinical trial to assess the efficacy of a comprehensive programme of secondary prevention of cardiovascular disease in general practice: the PREseAP study. Revista Española de Cardiología 2011;64(1):13-20.

Brotons C [pers comm]. Individual patient data used in the trial. Email to: K Lager 16 July 2012.

ISRCTN18578323. Randomised controlled trial to assess the efficacy of a comprehensive secondary prevention programme in primary care. www.controlled-trials.com/ISRCTN18578323 (first received 8 March 2005).

\section{Chanruengvanich 2006 \{published data only\}}

Chanruengvanich W, Kasemkitwattana S, Charoenyooth C, Towanabut S, Pongurgsorn C. Self-regulated exercise program in transient ischemic attack and minor stroke patients. Thai Journal of Nursing Research 2006;10(3):165-78.

\section{Chiu 2008 \{published data only\}}

Chiu CC, Wu SS, Lee Py, Huang YC, Tan TY, Chang KC. Control of modifiable risk factors in ischemic stroke outpatients by pharmacist intervention: an equal allocation stratified randomized study. Journal of Clinical Pharmacy and Therapeutics 2008;33(5):529-35.

\section{Damush 2015 \{published data only\}}

Damush T, Myers L, Anderson J, Yu Z, Ofner S, Nicholas G, et al. The effect of a locally adapted, secondary stroke risk factor self-management program on medication adherence among veterans with stroke/TIA. Translational Behavioural Medicine 2016;6(3):457-68

\section{Dregan 2014 \{published data only\}}

Dregan A, van Staa T, McDermott L, McCann G, Ashworth M, Charlton J, et al. Cluster randomized trial in the general practice research database: 2 . secondary prevention after first stroke (eCRT study): study protocol for a randomized controlled trial. Trials 2012;13:181-5.

* Dregan A, van Staa T, McDermott L, McCann G, Ashworth M, Charlton J, et al. Point-of-care cluster randomized trial in stroke secondary prevention using electronic health records. Stroke 2014;45:2066-71

Gulliford M, van Staa T, McDermott L, McCann G, Charlton J, Dregan A. Cluster randomized trials utilizing primary care electronic health records: methodological issues in design, conduct, and analysis (eCRT Study). Trials 2014;15:220-9.

ISRCTN35701810. Secondary prevention after first stroke. www.isrctn.com/ISRCTN35701810 (first received 16 March 2010).

\section{Eames 2013 \{published and unpublished data\}}

ACTRN12608000469314. Do stroke clients and carers provided with a post-discharge education and support package demonstrate better stroke knowledge as compared with those receiving usual care? www.anzctr.org.au/Trial/Registration/ TrialReview.aspx?ACTRN=12608000469314 (first received 26 August 2008).

Eames S, Hoffmann T, Worrall L, Read S, Wong A. Randomised controlled trial of a post-discharge education and support 
package for clients with stroke and their carers. BMJ Open 2013;3(5):e002538.

Eames S, Hoffmann T, Worrall L, Wong A, Read S. Evaluation of an innovative post-discharge education and support package for patients with stroke and their carers. International Journal of Stroke 2010;5(Suppl 2):190 (Abstract P010411).

* Eames S, Hoffman T, Worrall L, Read S, Wong A. Randomised controlled trial of an education and support package for stroke patients and their carers. BMJ Open 2013;3(5):e002538.

Eames S, Hoffman T, Worrall L, Read S, Wong A. Randomised controlled trial of a postdischarge education and support package for clients with stroke and their carers. Australian Occupational Therapy Journal 2011;58(2):51.

UK trialists. Individual patient data (as supplied 21 July 2012). Unpublished data on file.

\section{Ellis 2005 \{published and unpublished data\}}

Ellis G, Rodger J, McAlpine C, Langhorne P. Patient-centered education lowers blood pressure. Stroke 2004;35(1):257 (Abstract P25).

Ellis G, Rodger J, McAlpine C, Langhorne P. The impact of a stroke nurse specialist on risk factor modification in a TIA clinic: a randomised controlled trial. Age and Ageing 2004;33(Suppl 1):10.

* Ellis G, Rodger J, McAlpine C, Langhorne P. The impact of stroke nurse specialist input on risk factor modification: a randomised controlled trial. Age and Ageing 2005;34(4):389-92.

McManus J, Craig A, McAlpine C, Langhorne P, Ellis G. Does behaviour modification affect post-stroke risk factor control? Three-year follow-up of a randomized controlled trial. Clinical Rehabilitation 2009;23(2):99-105.

McManus JA, Craig A, Ellis G, McAlpine C, Langhorne P. 3 years on: does behaviour modification affect post stroke risk factor control? Age and Ageing 2006;35(Suppl 3):i72.

McManus JA, Craig A, Ellis G, McAlpine C, Langhorne P. 3 years on: does behaviour modification affect post stroke risk factor control? Cerebrovascular Diseases 2006;21(Suppl 4):92 (Abstract 12).

\section{Evans 2010 \{published and unpublished data\}}

Evans C [pers comm]. Outcome data for 13 subjects with stroke/ TIA in an SPSS file. Email to: K Lager June 2012.

Evans CD, Eurich DT, Taylor JG, Blackburn DF. The collaborative cardiovascular risk reduction in primary care (CCARP) study. Pharmacotherapy 2010;30(8):766-75.

\section{Flemming 2013 \{published and unpublished data\}}

Flemming K, Allison T, Covalt J, Herzig D, Brown R. Utility of a Post-Hospitalization Stroke Prevention Program Managed by Nurse. Hospital Practice 2013;41(3):70.

Flemming K, Brown R. Utility of a physician directed, nurse based stroke prevention program. Neurology 2012;78 (Meeting Abstracts 1):P07.022.
* Flemming KD, Allison T, Covalt J, Herzig D, Brown RD. The utility of a nurse case managed ischemic stroke post hospitalization prevention program (as supplied 13 May 2013). Unpublished report.

\section{Hanley 2015 \{published data only\}}

Hanley J, Fairborther P, Krishan A, McCloughan L, Padfield P, Paterson $\mathrm{M}$, et al. Mixed methods feasibility study for a trial of blood pressure telemonitoring for people who have had stroke/ transient ischaemic attack (TIA). Trials 2015;16:117.

\section{Hedegaard 2014 \{published data only\}}

* Hedegaard U, Kjeldsen LJ, Pottegard A, Bak S. Multifaceted intervention including motivational interviewing to support medication adherence after stroke/transient ischemic attack: a randomized trial. Cerebrovascular Disease 2014;4(3):221-34.

NCT01684176. Tailored intervention to improve patient adherence to secondary stroke prevention medication. www.clinicaltrials.gov/ct2/show/NCT01684176 (first received 12 September 2012).

\section{Hornnes 2011 \{published and unpublished data\}}

Hornnes AN, Boysen G, Larsen K. The post stroke preventive trial (PREVENT). A randomised controlled trial nested in a cohort. In: Cerebrovascular Diseases 2008, 25(Supplement 2), editors(s). 17th European Stroke Conference. 2008 May 13 to 16; Nice, France. Nice: Cerebrovascular Diseases, 2008.

* Hornnes N, Larsen K, Boysen G. Blood pressure 1 year after stroke: the need to optimise secondary prevention. Journal of Stroke and Cerebrovascular Diseases 2011;20(1):16-23.

Hornnes N, Larsen K, Boysen G. Little change of modifiable risk factors 1 year after stroke: a pilot study. International Journal of Stroke 2010;5(3):157-62.

NCT00253097. The post stroke preventive trial (PREVENT). A RCT nested in a cohort study. www.clinicaltrials.gov/ct2/show/ NCT00253097 (first received 15 November 2005).

\section{Johnston 2010 \{published and unpublished data\}}

Hills NK, Nguyen-Huynh M, Grosvenor D, Sidney S, Kingman J, Bernstein A, et al. Race and blood pressure control six months after ischemic stroke. Stroke 2008;39(2):622-3 (Abstract P213).

Johnston SC, Sidney S, Hills NK, Grosvenor D, Klingman JG, Bernstein A, et al. Standardised discharge orders after stroke: results of the quality improvement in stroke prevention (QUISP) trial. Stroke 2010;41(4):e289 (Abstract 130).

* Johnston SC, Sidney S, Hills NK, Grosvenor D, Klingman JG, Bernstein A, Levin E. Standardised discharge orders after stroke: results of the quality improvement in stroke prevention (QUISP) cluster randomised trial. Annals of Neurology 2010;67(5):579-89.

Kwan J, Johnston SC. The impact of standardised stroke orders on adherence to best practices. Neurology 2006;66(8):1130.

Navi BB, Kamel H, Sidney S, Hills NK, Nguyen-Huynh MN, Johnston SC. Statin cessation after ischaemic stroke. Stroke 2010;41(4):E244 (Abstract 157). 
NCT00328640. Quality improvement in stroke prevention (QUISP). www.clinicaltrials.gov/ct2/show/NCT00328640 (first received 22 May 2006).

\section{Jönsson 2014 \{published data only\}}

Jönsson A, Höglund P, Brizzi M, Pessah-Rasmussen $H$. Secondary prevention and health promotion after stroke: can it be enhanced? Journal of Stroke and Cerebrovascular Diseases 2014;23(9):2287-95.

Jönsson A [pers comm]. The standard deviation for the pre and post systolic and diastolic blood pressure results. BMI values pre and post intervention and their corresponding standard deviations using in the trial. Email to: B Bridgwood 01 August 2016.

NCT01466907. A secondary prevention and health promotion after stroke. www.clinicaltrials.gov/ct2/show/NCT01466907 (first received 8 November 2011).

Trialists. Individual patient data (as supplied 1 August 2016). Unpublished data on file.

\section{Joubert 2009 \{published data only\}}

ACTRN12611000264987. Reducing disability in older Australians through secondary stroke prevention. ICARUSS. www.anzctr.org.au/Trial/Registration/TrialReview.aspx? ACTRN=12611000264987 (first received 23 February 2011).

Joubert J, Joubert L, Jackson D, Wilson A, Pearce C, Reid C, et al. Improvement in risk factor management stroke survivors exposed to an integrated model of care. International Journal of Stroke 2010;5(1):27.

Joubert J, Joubert L, Reid C, Barton D, Cumming T, Mitchell P, et al. The positive effect of integrated care on depressive symptoms in stroke survivors. Cerebrovascular Diseases 2008;26(2):199-205.

Joubert J, Joubert LB, Reid C, Barton D, Cumming T, Mitchell P, et al. The positive effect of integrated care on depressive symptoms in stroke survivors. International Journal of Stroke 2008;3(Suppl 1):144 (Abstract 3).

* Joubert J, Reid C, Barton D, Cumming T, McLean A, Joubert L, et al. Integrated care improves risk-factor modification after stroke: initial results of the integrated care for the reduction of secondary stroke model. Journal of Neurology, Neurosurgery and Psychiatry 2009;80(3):279-84.

Joubert J, Reid C, Joubert L, Barton D, Ruth D. Ischemic Stroke: A Treatable Emergency and a Preventable Catastrophe 2nd International Workshop, Madrid, April 2002 (Supplement Issue: Cerebrovascular Diseases 2004, 1). Cerebrovascular Diseases 2004;17(Suppl 1):Meeting abstracts.

Joubert J, Reid C, Joubet L, Barton D, Ruth D, Jackson D, et al. Risk factor management and depression post-stroke: the value of an integrated model of care. Journal of Clinical Neuroscience 2006;13(1):84-100.

Joubert J. Shared care in stroke survivors - translation of research into service. Journal of the Neurological Sciences 2005;238(Suppl 1):S63 (Abstract OPL069).

\section{Kerry 2013 \{published data only\}}

Kerry S, Cloud G, Markus H, Khong T, Oakeshott P. Does self monitoring improve blood pressure control in hypertensive stroke patients - first results of a randomised trial. International Journal of Stroke 2010;5(Suppl 3):6.

Kerry S, Markus H, Khong T, Doshi R, Conroy R, Oakeshott P. Community based trial of home blood pressure monitoring with nurse-led telephone support in patients with stroke or transient ischaemic attack recently discharged from hospital. Trials 2008;9:15.

* Kerry SM, Markus HS, Khong TK, Cloud GC, Tulloch J, Coster D, et al. Home blood pressure monitoring with nurseled telephone support among patients with hypertension and a history of stroke: a community-based randomized controlled trial. Canadian Medical Association Journal 2013;185(1):23-31.

NCT00514800. Home Blood Pressure Monitoring Trial. clinicaltrials.gov/ct2/show/NCT00514800 (first received 10 August 2007).

\section{Kim 2013 \{published data only\}}

Kim JI, Lee S, Kim JH. Effects of a web-based stroke education program on recurrence prevention behaviors among stroke patients: a pilot study. Health Education Research 2013;28(3):488-501.

\section{Kono 2013 \{published data only\}}

Kono Y, Yamada S, Yamaguchi J, Hagiwara Y, Iritani N, Ishida S, et al. Secondary prevention of new vascular events with lifestyle intervention in patients with noncardioembolic mild ischemic stroke: a single-center randomized controlled trial. Cerebrovascular Diseases 2013;36(2):88-97.

UMIN000001865. Lifestyle intervention for prevention of stroke recurrence in mild stroke - a randomized controlled trial. rctportal.niph.go.jp/en/detail?trial_id=UMIN000001865 (first received 10 April 2009).

\section{Kronish 2014 \{published data only\}}

Goldfinger J, Kronish I, Fei K, Graciani A, Rosenfeld P, Lorig K, et al. Peer education for secondary stroke prevention in inner-city minorities: design and methods of the prevent recurrence of all inner-city strokes through education randomized controlled trial. Contemporary Clinical Trials 2012;33(5):1065-73.

* Kronish I, Goldfinger J, Negron R, Fei K, Tuhrim S, Arniella G, et al. Effect of peer education on stroke prevention. The prevent recurrence of all inner city strokes through education randomized controlled trial. Stroke 2014;45(11):3330-6.

NCT01027273. Prevent return of stroke study. www.clinicaltrials.gov/ct2/show/NCT01027273 (first received 7 December 2009).

\section{Lowe 2007 \{published and unpublished data\}}

ISRCTN55373356. The CareFile project: an assessment of the impact of individualised information booklets in patients post stroke. www.controlled-trials.com/ISRCTN55373356 (first received 11 September 2005). 
Lowe D, Leathley M, Sharma A. Patient education following stroke: the CareFile project. Cerebrovascular Diseases 2002;13(Suppl 3):81.

Lowe D, Leathley M, Sharma A. The effects of an individualised booklet in post stroke patients: the care file project.

Cerebrovascular Diseases 2003;16(Suppl 4):95 (Abstract P404).

Lowe D, Leathley MJ, Sharma AK. An assessment of the utility of an individualised information booklet in patients after stroke: the CAREFILE project. Age and Ageing 2005;34(Suppl 1):i38.

Lowe DB, Leathley MJ, Sharma AK. Assessment of stroke knowledge. Age and Ageing 2002;31(Suppl 1):42.

* Lowe DB, Sharma AK, Leathley MJ. The CareFile project: a feasibility study to examine the effects of an individualised information booklet on patients after stroke. Age and Ageing 2007;36(1):83-9.

\section{Lowrie 2010 \{published and unpublished data\}}

* Lowrie R, Morrison J, McConachie A. A cluster randomised controlled trial of pharmacist led statin outreach support (SOS) in primary care: design and baseline characteristics. Contemporary Clinical Trials 2010;31(4):303-11.

UK trialists. Summary patient data (as supplied 8 July 2013). Unpublished data on file.

\section{Maasland 2007 \{published and unpublished data\}}

* Maasland E, Koudstaal PJ, Habbema JDF, Dippel DWJ. Effects of an individualized multimedia computer program for health education inpatients with a recent minor stroke or transient ischaemic attack - a randomized controlled trial. Acta Neurologica Scandinavica 2007;115(1):41-8.

Maasland L, Koudstall PJ, Habbema JDF, Dippel DWJ. Knowledge and understanding of disease process, risk factors and treatment modalities in patients with recent TIA or minor ischaemic stroke. Cerebrovascular Diseases 2007;23(2-3):435-40.

MacKenzie 2013 \{published data only\}

* Mackenzie G, Ireland S, Moore S, Heinz I, Johnson R, Oczkowski W, et al. Tailored interventions to improve hypertension management after stroke or TIA - phase II (TIMS II). Canadian Journal of Neuroscience Nursing 2013;35(1):27-34.

Mackenzie G, Ireland S, Sahlas D, Oczkowski W, Gould L, LeBlanc K, et al. Tailored interventions to improve hypertension in management after stroke. Stroke 2011;42(11):e599.

\section{Mant 2016 \{published data only\}}

Fletcher K, Mant J, McManus R, Campbell S, Betts J, Taylor C, et al. Protocol for Past BP: a randomised controlled trial of different blood pressure targets for people with a history of stroke of transient ischaemic attack (TIA) in primary care. BMC Cardiovascular Disorders 2010;10(1):37.

* Mant J, McManus R, Roalfe A, Fletcher K, Taylor C, Martin U, et al. Different systolic blood pressure targets for people with history of stroke or transient ischaemic attack: PAST$\mathrm{BP}$ (prevention after stroke - blood pressure) randomised controlled trial. BMJ 2016;352:i708. [DOI: 10.1136/bmj.i708]

\section{Markle-Reid 2011 \{published and unpublished data\}}

* Markle-Reid M, Orridge C, Weir R, Browne G, Gafni A, Lewis M, et al. Interprofessional stroke rehabilitation for stroke survivors using home care. Canadian Journal of Neurological Sciences 2011;38(1):317-34.

NCT00463229. Interdisciplinary team approach to stroke rehabilitation in home care. www.clinicaltrials.gov/ct2/show/ NCT00463229 (first received 20 April 2007).

McAlister 2014 \{published data only\}

McAlister F, Grover S, Padwal J, Youngson E, Fradette M, Thompson A, et al. Case management reduces global vascular risk after stroke: secondary results from the preventing recurrent vascular events and neurological worsening through intensive organized case-management randomized controlled trial. American Heart Journal 2014;168(6):924-30.

* McAlister F, Majumdar S, Padwal R, Fradette M, Thompson A, Buck $B$, et al. Case management for blood pressure and lipid level control after minor stroke: PREVENTION randomized controlled trial. Canadian Medical Association Journal 2014;186(8):577-84.

McAlister F, Majumdar S, Padwal R, Fradette M, Thompson A, Tsuyuki $R$, et al. The preventing recurrent vascular events and neurological worsening through intensive organized casemanagement (PREVENTION) trial protocol. Implementation Science 2015;5:27.

NCT00931788. Preventing recurrent vascular events in patients with stroke or transient ischemic attack (PREVENTION). www.clinicaltrials.gov/ct2/show/NCT00931788 (first received 2 July 2009).

\section{McManus 2014 \{published data only\}}

* McManus R, Mant J, Haque S, Bray E, Bryan S, Greenfield S, et al. Effect of self-monitoring and medication self-titration on systolic blood pressure in hypertensive patients at high risk of cardiovascular disease. The TASMIN-SR randomized clinical trial. JAMA 2014;312(8):799-808.

McManus R, Ryan R, Jones M, Wilson S, Hobbs F. How representatives of primary care are research active practices? Cross sectional survey. Family Pratice 2008;25(1):56-62.

O'Brien C, Bray E, Bryan S, Greenfield S, Haque S, Hobbs R, et al. Targets and self-management for the control of blood pressure in stroke and at risk groups (TASMIN-SR): protocol for a randomised controlled trial. BMC Cardiovascular Disease 2013;13:21.

Sheppard J [pers comm]. Blood pressure values use in trial. Email to: B Bridgwood 25 October 2016.

Trialists. Individual patient data (as supplied 1 November 2016). Unpublished data on file.

\section{MIST 2014 \{published data only\}}

ACTRN12610000715077. Motivational interviews for secondary stroke prevention: a randomised clinical trial. anzctr.org.au/ Trial/Registration/TrialReview.aspx?ACTRN=12610000715077 (first received 27 August 2010). 
Barker-Collo S, Barber P, Witt E, Feigin V, Jones A, McPherson K. Improving adherence to secondary stroke prevention strategies through motivational interviewing: a randomised controlled trial. Stroke 2016;47(1):WP405.

* Barker-Collo S, Krishnamurthi R, Witt E, Feigin V, Jones A, McPherson K, et al. Improving adherence to secondary stroke prevention strategies through motivational interviewing randomized controlled trial. Stroke 2015;46(12):3451-8.

Barker-Collo S, Krishnamurthi R, Witt E, Feigin V, Jones A, McPherson K. Improving adherence to secondary stroke prevention strategies through motivational interviewing: randomized controlled trial. www.anzctr.org.au/] [Ref 24682].

Krishnamurthi R, Witt E, Barker-Collo S, McPherson K, Davis-Martin K, Bennett $\mathrm{D}$, et al. Reducing recurrent stroke: methodology of the motivational interviewing in stroke (MIST) randomized clinical trial. Stroke 2014;9(1):133-9.

\section{Nailed Stroke 2010 \{published data only\}}

* Irewall A, Ögren J, Bergström L, Laurell K, Söderström L, Mooe T. Nurse-Led, telephone-based, secondary preventive follow-up after stroke or transient ischemic attack improves blood pressure and LDL cholesterol: results from the first 12 months of the randomized controlled NAILED stroke risk factor trial. PloS One 2015;10(10):e0139997.

Irewall AL, Johansson C, Stromvall A, Mooe T. Nurse-led, telephone-based secondary preventative intervention after stroke or TIA improves blood pressure after 12 months of follow-up. International Journal of Stroke 2014;9(3):278-9 WSC-1531.

ISRCTN23868518. Secondary preventive, nurse-based, telephone follow-up for risk factor control after stroke or transient ischemic attack. www.isrctn.com/ISRCTN23868518 (first received 13 May 2012).

Mooe T, Bergström L, Irewall A, Ögren J. The NAILED stroke risk factor trial (nurse based age independent intervention to limit evolution of disease after stroke): study protocol for randomized controlled trial. Trials 2013;14:5.

\section{O'Carroll 2011 \{published and unpublished data\}}

ISRCTN38274953. Improving adherence to medication in stroke survivors: a single centre randomised controlled pilot study. www.controlled-trials.com/ISRCTN38274953 (first received 3 December 2009).

* O'Carroll R, Chambers J, Dennie M, Sudlow C, Johnston M. Improving adherence to medication in stroke survivors: a pilot randomised controlled trial. Annuals of Behavioural Medicine 2013;46(3):358-68.

O'Carroll R, Whittaker J, Hamilton B, Johnston M, Sudlow C, Dennis M. Predictors of adherence to secondary preventive medication in stroke patients. Annals of Behavioral Medicine 2011;41(3):383-90.

O'Carroll R. Improving adherence to medication in stroke survivors (IAMSS): a randomised controlled trial: study protocol. BMC Neurology 2010;10:15.
UK trialists. IAMSS Final Report (as supplied 18 April 2013). Unpublished report.

Peng 2014 \{published data only\}

NCT00664846. Standard medical management in secondary prevention of ischemic stroke in China (SMART). www.clinicaltrials.gov/ct2/show/NCT00664846 (first received 23 April 2008).

* Peng B, Ni J, Anderson C, Zhu Y, Wang Y, Pu C, et al. Implementation of a structured guideline-based program for the secondary prevention of ischemic stroke in China. Stroke 2014;45(2):515-9.

Peng B, Zhu Y, Cui L, Ni J, Xu W, Zhou L, et al. Standard medical management in secondary prevention of ischemic stroke in China (SMART). International Journal of Stroke 2011;6(5):461-5.

Pergola 2014 \{published data only\}

Benavente O, White C, Pearce L, Pergola P, Roldan A, Benavente $\mathrm{M}$, et al. The secondary prevention of small subcortical strokes (SPS3) study. International Journal of Stroke 2011;6(2):161-75.

* Pergola P, White C, Szychowski J, Talbert R, del Bruto O, Castellanos M, et al. Achieved blood pressures in the secondary prevention of small subcortical strokes (SPS3) study: challenges and lessons learned. American Journal of Hypertension 2014;27(8):1052.

White C, Szychowski J, Roldan A, Benavente M, Pretell E, Del Brutto $O$, et al. Clinical features and racial/ethnic differences among the 3020 participants in the secondary prevention of small subcortical strokes (SPS3) trial. Journal of Stroke and Cerebrovascular Diseases 2013;22(6):767-74.

\section{Ranta 2015 \{published data only\}}

ACTRN12611000792921. Efficacy and safety of a transient ischaemic attack (TIA) electronic support tool [Multi-centre randomised controlled trial comparing the effect of general practitioner utilisation of a TIA electronic decision support tool in the management of TIA/minor stroke patients versus usual care on the subsequent 90 day stroke risk, degree of guideline adherence, and overall treatment costs]. www.anzctr.org.au/ Trial/Registration/TrialReview.aspx?id=343255\&isReview=true (first received 26 July 2011).

* Ranta A, Dovey S, Weatherall M, O'Dea D, Gommans J, Murray T. Cluster randomized controlled trial of TIA electronic decision support in primary care. American Academy of Neurology 2015;84(1):1545.

Ranta A, Dovey S, Weatherall M, O'Dea D. Efficacy and safety of a TIA/stroke electronic support tool (FASTEST) trial: study protocol. Implemental Science 2012;7:107.

Ranta A, Yang C-F, Funnell M. Utility of primary care based transient ischaemic attack electronic decision support tool: a prospective sequential comparison. BMC Family Practice 2014;155:85. 
Slark 2013 \{published and unpublished data\}

ISRCTN67999605. Individual risk awareness intervention in stroke. www.controlled-trials.com/ISRCTN67999605 (first received 5 August 2010).

* Slark J, Khan MS, Bentley P, Sharma P. Individual risk awareness intervention in stroke (IRAIS): a randomised controlled trial (as supplied 17 June 2013). Unpublished report.

Slark J, M, Khan, Bentley P, Sharma P. Individual risk awareness intervention in stroke (IRAIS): a randomized controlled trial. Journal of Neurology Disorders and Stroke 2013;2(2):1035.

Slark JS. Risk awareness in secondary stroke prevention. London: Imperial College London, 2012.

\section{Wan 2016 \{published data only\}}

ChiCTR-IPR-15005946. Efficacy of comprehensive reminder system based on health belief model on secondary prevention of hypertensive ischemic stroke. www.chictr.org.cn/ showprojen.aspx?proj=10388 (first received 3 February 2015).

* Wan L, Zhang X, Mo M, Xiong X, Ou C, You L, et al. Effectiveness of goal-setting telephone follow-up on health behaviors of patients with ischemic stroke: a randomized controlled trial. Journal of Stroke and Cerebrovascular Diseases 2016;25(9):2259.

Wang 2005 \{published data only\}

Wang L. Neurologist participates in the community intervention for the functional prognosis of convalescent patients with stroke. Chinese Journal of Clinical Rehabilitation 2005;9(17):4-5.

\section{Welin 2010 \{published and unpublished data\}}

NCT00976001. Follow-up pilot study after a first stroke. www.clinicaltrials.gov/ct2/show/NCT00976001 (first received 14 September 2009).

* Welin L, Bjälkefur K, Roland I. Open, randomized pilot study after first stroke: a 3.5 year follow-up. Stroke 2010;41(7):1555-7.

\section{References to studies excluded from this review}

\section{Amariles 2012 \{published data only\}}

Amariles $P$, Sabater-Hernández D, García-Jiménez E, RodríguezChamorro MÁ, Prats-Más R, Marín-Magán F, et al. Effectiveness of Dader method for pharmaceutical care on control of blood pressure and total cholesterol in outpatients with cardiovascular disease or cardiovascular risk: EMDADER-CV randomized controlled trial. Journal of Managed Care Pharmacy 2012;18(4):311-23.

\section{Banet 1997 \{published data only\}}

Banet GA, Felchlia MA. The potential utility of a shared medical record in a "first-time" stroke population. Journal of Vascular Nursing 1997;15(1):29-33.

\section{Bokemark 1996 \{published data only\}}

Bokemark L, Blomstrand C, Fagerberg B. Considerable differences in the management of stroke. A study of structured vs. conventional care. Lakartidningen 1996;93(8):681-5.
FIMDM_CVD 2010 \{published data only\}

NCT01134458. Personalised cardiovascular risk information to initiate and maintain behavior changes (FIMDM_CVD). clinicaltrials.gov/show/NCT01134458 (first received 2 June 2010).

* Powers BJ, Danus S, Grubber JM, Olsen MK, Oddone EZ, Bosworth HB. The effectiveness of personalised coronary heart disease and stroke risk communication. American Heart Journal 2011;161(4):673-80.

Zullig L, Stechuchak K, Goldstein K, Olsen M, McCant F, Danus S, et al. Patient-reported medication adherence barriers among patients with cardiovascular risk factors. Journal of Managed Care and Specialty Pharmacy 2015;21(6):479-85.

Gillham 2010 \{published data only\}

* Gillham S, Endacott R. Impact of enhanced secondary prevention on health behaviour in patients following minor stroke and transient ischaemic attack: a randomised controlled trial. Clinical Rehabilitation 2010;24(9):822-30.

Gillham SD. Does enhanced secondary prevention intervention after minor stroke and transient ischaemic attack (TIA) affect readiness to change health behaviour? Conference: 4 th UK Forum Stroke Conference. Glasgow, United Kingdom, 2009. International Journal of Stroke 2009;4:27.

Goessens 2006 \{published data only\}

${ }^{*}$ Goessens BMB, Visseren FLJ, Sol BGM, de Man-van Ginkel JM, van der Graaf Y. A randomised, controlled trial for risk factor reduction in patients with symptomatic vascular disease: the multidisciplinary vascular prevention by nurses study (VENUS). European Journal of Cardiovascular Prevention and Rehabilitation 2006;13(6):996-1003.

Sol BG, van der Graaf Y, van der Bijl JJ, Goessens BM, Visserten FL. The role of self-efficacy in vascular risk factor management: a randomised controlled trial. Patient Education and Counseling 2008;71(2):191-7.

Green 2007 \{published data only\}

* Green T, Haley E, Eliasziw M, Hoyte K. Education in stroke prevention: efficacy of an educational counselling intervention to increase knowledge in stroke survivors. Canadian Journal of Neuroscience Nursing 2007;29(2):13-20.

Green T, Haley E, Eliasziw M. Patient education in stroke prevention. Stroke 2006;37(2):688 (Abstract P211).

\section{Harrington 2007 \{published data only\}}

Harrington R, Taylor G, Duggan A, Reed M, Wood V. The evaluation of a community-based stroke scheme. Disability and Rehabilitation 2007;29(20-21):1636-7.

\section{Johnston 2000 \{published data only\}}

Johnston B. Outcomes of the Kaiser Permanente telehome health research project. Archives of Family Medicine 2000;9(1):40-5. 
Joshi 2012 \{published data only\}

Chow CK, Joshi R, Gottumukkala AK, Raju K, Raju R, Reddy S, et al. Rationale and design of the rural Andhra Pradesh cardiovascular prevention study (RAPCAPS): a factorial, cluster-randomised trial of 2 practical cardiovascular disease prevention strategies developed for rural Andhra Pradesh, India. American Heart Journal 2009;158(3):349-55.

* Joshi R, Chow C, Raju K, Raju R, Gottumukkala AK, Reddy S, et al. The rural Andhra Pradesh cardiovascular prevention study (RAPCAPS): a cluster randomised trial. Journal of the American College of Cardiology 2012;59(13):1188-96.

NCT00263393. Rural Andhra Pradesh cardiovascular prevention study (RAPCAPS). clinicaltrials.gov/show/NCT00263393 (first received 8 December 2005).

\section{Ma 2009 \{published data only\}}

* Ma J, Berra K, Haskell WL, Klieman L, Hyde S, Smith MW, et al. Case management to reduce cardiovascular risk in a county healthcare system. Archives of Internal Medicine 2009;169(21):1988-95.

Ma J, Lee K, Berra K, Stafford RS. Implementation of case management to reduce cardiovascular disease risk in the Stanford and San Mateo heart to heart randomised controlled trial: study protocol and baseline characteristics. Implementation Science 2006;1:21.

NCT00127751. Heart disease on the mend. clinicaltrials.gov/ show/NCT00127751 (first received 8 August 2005).

\section{Middleton 2004 \{published data only\}}

Middleton S, Donnelly N, Harris J, Ward J. Nursing intervention after carotid endarterectomy: a randomised trial of coordinated care post-discharge. Issues and Innovations in Nursing Practice 2004;52(3):250-61

\section{Nir 2006 \{published data only\}}

Nir Z, Weisel-Eichler A. Improving knowledge and skills for use of medication by patients after stroke: evaluation of a nursing intervention. American Journal of Physical Medicine and Rehabilitation 2006;85(7):582-92.

\section{Ornstein 2004 \{published data only\}}

* Ornstein S, Jenkins RG, Nietert PJ, Feifer C, Roylance LF, Nemeth L, et al. A multimethod quality improvement intervention to improve preventive cardiovascular care: a cluster randomised trial. Annals of Internal Medicine 2004;141(7):523-32.

Ornstein SM. Translating research into practice using electronic medical records the PPRNet-TRIP project: primary and secondary prevention of coronary heart disease and stroke. Topics in Health Information Management 2001;22(2):52-8.

\section{Palanco 2011 \{published data only\}}

* Moreno-Palanco MA, Ibanez-Sanz P, Ciria-de Pablo C, Pizarro-Portillo A, Rodriquez-Salvanes F, Suarez-Fernandez C. Impact of comprehensive and intensive treatment of risk factors concerning cardiovascular mortality in secondary prevention: MIRVAS study. Revista Española de Cardiología 2011;64(3):179-85.

Palanco MAM, de Pablo CC, Sanz IP, Luis SC, Portillo AP, Fernandez C. Cardiovascular morbimortality reduction after an acute cardiovascular event through multifactorial and intensive cardiovascular risk factors management (MIRVAS project). Medicina Clinica 2007;129(7):241-6.

\section{Rimmer 2000 \{published data only\}}

Rimmer JH, Braunschweig C, Silverman K, Riley B, Creviston T, Nicola T. Effects of a short-term health promotion intervention for a predominantly African-American group of stroke survivors. American Journal of Preventive Medicine 2000;18(4):332-8.

\section{Ross 2007 \{published data only\}}

Ross MA, Compton S, Medado P, Fitzgerald M, Kilanowski P, O'Neil BJ. An emergency department diagnostic protocol for patients with transient ischaemic attack: a randomized controlled trial. Annals of Emergency Medicine 2007;50(2):109-19.

\section{Sides 2012 \{published data only\}}

Bushnell C, Zimmer L, Schwamm L, Goldstein LB, ClappChanning N, Harding T, et al. The adherence evaluation after ischaemic stroke longitudinal (AVAIL) registry: design, rationale, and baseline patient characteristics. American Heart Journal 2009;157(3):428-35.

NCT01115660. Stroke education intervention trial - pilot AVAIL II. clinicaltrials.gov/show/NCT01115660 (first received 24 January 2013).

* Sides EG, O Zimmer L, Wilson L, Pan W, Olson DM, Peterson ED, et al. Medication coaching program for patients with minor stroke or TIA: a pilot study. BMC Public Health 2012;12:549.

\section{Spassova 2016 \{published data only\}}

Spassova L, Vittore D, Droste D, Rosch N. Randomised controlled trial to evaluate the efficacy and usability of a computerised phone-based lifestyle coaching system for primary and secondary prevention of stroke. BMC Neurology 2016;16:22.

\section{Strandberg 2006 \{published data only\}}

Strandberg TE, Pitkala K, Berglind S, Nieminen MS, Tilvis RS. Multifactorial cardiovascular disease prevention in patients aged 75 years and older: a randomised controlled trial: drugs and evidence based medicine in the elderly (DEBATE) study. American Heart Journal 2001;142(6):945-51.

Strandberg TE, Pitkala K, Berglind S, Nieminen MS, Tilvis RS. Multifactorial cardiovascular prevention in patients aged 75 and over: design and baseline results of a randomised controlled trial (DEBATE Study). European Heart Journal 2001;22(6):460 (Abstract P2408).

Strandberg TE, Pitkala K, Berglind S, Nieminen MS, Tilvis RS. Possibilities of multifactorial cardiovascular disease prevention in patients aged 75 and older: a randomised controlled trial. 
Drugs and evidence based medicine in the elderly (DEBATE) study. European Heart Journal 2003;24(13):1216-22.

* Strandberg TE, Pitkala KH, Berglind S, Nieminen MS, Tilvis RS. Multifactorial intervention to prevent recurrent cardiovascular events in patients 75 years or older: the drugs and evidencebased medicine in the elderly (DEBATE) study: a randomised, controlled trial. American Heart Journal 2006;152(4):585-92.

\section{UMIN000001865 \{published and unpublished data\}}

UMIN000001865. Lifestyle intervention for prevention of stroke recurrence in mild stroke - a randomised controlled trial. http:// apps.who.int/trialsearch/Trial.aspx?TrialID=UMIN000001865 (first received 10 April 2009).

\section{Vernooij 2012 \{published data only\}}

Vernooij JW, Kaasjager HA, van der Graaf Y, Wierdsma J, Grandjean HM, Hovens MM, et al. Internet based vascular risk factor management for patients with clinically manifest vascular disease: randomised controlled trial. BMJ 2012;344:e3750.

\section{References to studies awaiting assessment}

ACTRN12608000166370 \{published data only\}

ACTRN12608000166370. Shared team approach between nurses and doctors for improved risk factor management for stroke patients. www.anzctr.org.au/Trial/Registration/ TrialReview.aspx?id=82711 (first received 31 March 2008).

\section{Feld-Glazman 2012 \{published data only\}}

Feld-Glazman R, Bushnik T, Van Lew S, Sheikovitz L. The impact of a stroke education program on patient's stroke knowledge and their change of stroke risk behaviors. Archives of Physical Medicine and Rehabilitation 2012;93(10):E39.

\section{ISRCTN63816609 \{published data only\}}

ISRCTN63816609. Improving the prevention of vascular events after stroke or transient ischemic attack: a randomised controlled pilot trial of nurse independent prescriber-led care pathway-based risk factor management. isrctn.com/ ISRCTN63816609 (first received 7 May 2012).

\section{ISRCTN95662526 \{published data only\}2834010}

* ISRCTN95662526. The you call - we call trial: impact of a multimodal support intervention after a "mild" stroke. www.controlled-trials.com/ISRCTN95662526 (first received 11 July 2008).

Rochette A, Korner-Bitensky N, Bishop D, Teasell R, White C, Bravo G, et al. Study protocol of the you call - we call trial: impact of a multimodal support intervention after a "mild" stroke. BMC Neurology 2010;10:3.

Rochette A, Korner-Bitensky N, White C, Bravo G, Côté R, Green T, et al. You all - we call trial: impact of a multimodal support intervention after a "mild" stroke. Stroke 2013;44:ANS13.
NCT00211731 \{published and unpublished data\}

* Cowles C, Tuhrim S, Brust J, Koppel B, Weinberger J, Horowitz $\mathrm{C}$, et al. Participation in peer-led support groups improves use of secondary stroke prevention measures. Neurology 2008;70(11 Suppl 1):A203.

NCT00211731. Preventing recurrent stroke in minority populations. clinicaltrials.gov/ct2/show/NCT00211731 (first received September 2005).

NCT00703274 \{published and unpublished data\}

Dromerick AW, Gibbons C, Edwards DF, Farr D, Jayam-Trouth A, Shara NM, et al. A phase II RCT of stroke navigators to improve compliance with secondary stroke prevention: PROTECT DC (Abst. CTP33). In: Proceedings of the International Stroke Conference 2011. Los Angeles, USA: American Heart Association and American Stroke Association, 8-11 February 2011.

Dromerick AW, Gibbons MC, Covington C, Farr D, JayamTrouth A, Shara NM, et al. A phase II RCT of stroke navigators to improve compliance with secondary stoke prevention. PROTECT DC (Abst. CT P23). In: Proceedings of the International Stroke Conference 2009. California, USA: American Heart Association and American Stroke Association, 18-20 February 2009.

Dromerick AW, Gibbons MC, Edwards DF, Farr D, Sanchez BN, Fokar A, et al. A Phase II RCT of stroke navigators to improve compliance with secondary stroke prevention: PROTECT DC (Abst. CT P26). In: Proceedings of the International Stroke Conference 2010. Texas, USA: American Heart Association and American Stroke Assocation, 24-26 February 2010.

Dromerick AW, Gibbons MC, Edwards DF, Farr DE, Giannetti M, Sanchez B, et al. Preventing recurrence of thromboembolic events through coordinated treatment in the district of Columbia (PROTECT DC). International Journal of Stroke 2011;6(5):454-60.

* NCT00703274. Preventing recurrence of thromboembolic events through coordinated treatment in the district of Columbia (PROTECT DC). clinicaltrials.gov/show/NCT00703274 (first received 23 June 2008).

\section{NCT01071408 \{published and unpublished data\}}

Cheng EM, Cunningham WE, Towfighi A, Sanossian N, Bryg RJ, Anderson TL, et al. Randomized, controlled trial of an intervention to enable stroke survivors throughout the Los Angeles County safety net to "stay with the guidelines". Circulation. Cardiovascular Quality and Outcomes 2011;4(2):229-34.

* NCT01071408. Trial of a secondary stroke prevention program. clinicaltrials.gov/show/NCT01071408 (first received 19 February 2010).

\section{NCT01122394 \{published data only\}}

NCT01122394. Reducing risk of recurrence (RRR). clinicaltrials.gov/ct2/show/NCT01122394 (first received 13 May 2010). 


\section{NCT01807793 \{published data only\}}

NCT01807793. Targeted management intervention for AfricanAmerican men with TIA or stroke. clinicaltrials.gov/show/ NCT01807793 (first received 8 March 2013).

Sajatovic M, Tatsuoka C, Welter E, Colon-Zimmerman K, Blixen C, Perzynski A. A targeted self-management approach for reducing stroke risk factors in young African-American men who have experienced stroke or transient ischemic attack. Stroke 2016;47(1):TP421.

\section{NCT02140658 \{published data only\}}

Internet Stroke Centre. Health education interventions for statins medication compliance and clinical prognosis of ischemic stroke patients. www.strokecenter.org/trials/ clinicalstudies/health-education-interventions-for-statinsmedication-compliance-and-clinical-prognosis-of-ischemicstroke-patients (accessed prior to 19 February 2018).

\section{Redfern 2007 \{published data only\}}

ISRCTN10730637. The south London secondary prevention programme. www.controlled-trials.com/ISRCTN10730637 (first received 19 July 2005).

Redfern J, McKevitt C, Frisby L, Montoute K, Rudd A, Wolfe C. Stop stroke: the south London secondary prevention programme. Evaluation of a multifaceted secondary prevention programme for stroke. In: Proceedings of the 13th European Stroke Conference (www.eurostroke.org/ esc_ongoing_trials.asp). 13-15 May 2004. MannheimHeidelberg, Germany, 2004.

Redfern J, McKevitt C, Rudd AD, Heuschmann P, Grieve A, Wolfe CDA. Optimising secondary prevention post stroke - first results of the stop stroke study. In: Proceedings of the 3rd UK Stroke Forum Conference 2008. 2-4 December 2008. Harrogate, UK: The Stroke Association, 2008.

Redfern J, McKevitt C, Rudd AG, Heuschmann P, Grieve A, Wolfe CDA. Stop stroke: cluster randomised controlled trial of a patient/carer and general practitioner intervention to improve risk factor management after stroke. Cerebrovascular Diseases 2009;27(1):66.

Redfern J, Rudd AD, Wolfe CDA, McKevitt C. Stop stroke: development of an innovative intervention to improve risk factor management after stroke. Patient Education and Counseling 2008;72:201-9.

Redfern J, Wolfe C, McKevitt C. How does stop stroke work? Qualitative process evaluation of a multiple risk factor intervention to improve stroke secondary prevention. Cerebrovascular Diseases 2008;25(Suppl 2):189 (Abstract 7).

Redfern J. The stop stroke secondary prevention trial: one year update. In: Proceedings of the The Stroke Association 9th Scientific Conference. 15-16 September 2004. Cambridge, UK: The Stroke Association, 2004.

Refern J, McKevitt C, Rudd A, Wolfe C. Stop stroke. A new intervention to improve stroke secondary prevention (Abstract 18). In: Proceedings of the 12th European Stroke Conference. Valencia, Spain, 21-24 May 2003.
Refern J. Methods for developing and evaluating randomised controlled trials of complex interventions - case study of stroke secondary prevention. europepmc.org/theses/ETH/443795 2007.

Wolfe C. The south London stroke secondary prevention programme. In: Proceedings of the UK Stroke Forum Conference 2007. 4-6 December 2007. Harrogate, UK: The Stroke Association, 2007.

* Wolfe CD, Redfern J, Rudd AG, Grieve AP, Heuschmann PU, McKevitt C. Cluster randomized controlled trial of a patient and general practitioner intervention to improve the management of multiple risk factors after stroke: stop stroke. Stroke 2010;41(11):2470-6.

\section{References to ongoing studies}

\section{ACTRN12615000888561 \{published and unpublished data\}}

ACTRN12615000888561. A conversation with patients about medications after a stroke. www.anzctr.org.au/Trial/ Registration/TrialReview.aspx?id=368938 (first received 29/7/2015).

\section{ChiCTR-TQR-14004950 \{published data only\}}

ChiCTR-TQR-14004950. Construction of "hospital-CommunityFamily" transitional care model for elderly hypertensive patients based on information platform. chictr.org.cn/ showprojen.aspx?proj=4623 (first received 6 July 2014).

ChiCTR-TRC-12002127 \{published and unpublished data\} ChiCTR-TRC-12002127. Effects of clinical pharmacist interventions on the secondary prevention in the ischemic stroke patients. apps.who.int/trialsearch/Trial.aspx? TrialID=ChiCTR-TRC-12002127 (first received 8 September 2015).

\section{COACH 2014 \{published data only\}}

NCT02207023. Healthy lifestyles after stroke (Stroke Coach). clinicaltrials.gov/ct2/show/NCT02207023 (first received 1 August 2014).

* Sakakibara B, Eng J, Benavente O, Barr S, Silverberg N, Goldsmith C. A telehealth intervention to promote healthy lifestyles after stroke: the Stroke $\mathrm{COACH}$ protocol. Stroke 2014;4(12):e285.

\section{DESERVE 2014 \{published data only\}}

* Benn E, Quarles L, Sofianou A, Perez V, Nieto V, BodenAlbala B. John Henryism, race-ethnicity and stroke risk: pilot findings from the DESERVE trial. Stroke 2014;45(1):Abst.ATMP64.

Boden-Albala B, Goldmann E, Lord A, Parikh N, Kuczynski H, Tuhrim S. A culturally-tailored, skills-based intervention to reduce vascular risk in a multi-ethnic group of mild/moderate stroke survivors: an interim analysis from the DESERVE trial. Stroke 2017;48(1):ATMP103.

Lord A, Carman H, Roberts E, Torrico E, Goldmann E, Ishida. Discharge educational strategies for reduction of vascular events (DESERVE): design and methods. International Journal of Stroke 2015;10(A100):151-4. 
NCT01836354. Discharge educational strategies for reduction of vascular events (DESERVE). clinicaltrials.gov/ct2/show/ NCT01836354 (first received 29 April 2013).

\section{DMP 2014 \{published data only\}}

* Fukuoka Y, Hosomi N, Hyakuta T, Omori T, Ito Y, Uemura J. Baseline feature of a randomized trial assessing the effects of disease management programs for the prevention of recurrent ischemic stroke. Journal of Stroke and Cerebrovascular Diseases 2015;24(3):610-7.

NCT02121327. The effects of disease management programs for prevention of recurrent ischemic stroke. clinicaltrials.gov/ct2/ show/NCT02121327 (first received 23 April 2014).

\section{Feldman 2015 \{published data only\}}

Feldman P, McDonald M, Trachtenberg M, Schoenthaler A, Coyne N, Teresi J. Center for stroke disparities solutions community- based care transition interventions: study protocol of a randomized controlled trial. Trials 2015;16:32.

\section{ISRCTN07607027 \{published and unpublished data\}}

* ISRCTN07607027. Promoting adherence to a regimen of risk factor modification by trained non-medical personnel evaluated against regular practice study PARTNERS. www.isrctn.com/ ISRCTN07607027 (first received 9 March 2009).

Mayer C, Chan R, Hachinski V. Partners promoting adherence to regimen of risk factor modification by trained volunteers or nurses evaluated against regular practice study. International Journal of Stroke 2008;3(Suppl 1):321 (Abstract PO02-212).

ISRCTN08913646 \{published and unpublished data\}

ISRCTN08913646. Study to determine whether the empowered stroke patients demonstrate better self-management behaviour and health outcomes. www.controlled-trials.com/ ISRCTN08913646 (first received 6 June 2012).

ISRCTN97412358 \{published data only\}

ISRCTN97412358. ECG monitoring to detect atrial fibrillation after stroke. www.controlled-trials.com/ISRCTN97412358 (first received 28 April 2010).

\section{NCT01517542 \{published and unpublished data\}}

NCT01517542. Evaluation of effectiveness of nutritional counselling in patients after stroke. www.clinicaltrials.gov/ show/NCT01517542 (first received 25 January 2012).

\section{NCT01586702 \{published and unpublished data\}}

Leistner S, Benik S, Laumeier I, Ziegler A, Nieweler G, Nolte CH, et al. Secondary prevention after minor stroke and TIA usual care and development of a support program. PloS One 2012; 7(12):e49985.

Leistner S, Michelson G, Laumeier I, Ahmadi M, Smyth M, Nieweler $\mathrm{G}$, et al. Intensified secondary prevention intending a reduction of recurrent events in TIA and minor stroke patients (INSPiRE-TMS): a protocol for a randomised controlled trial. BMC Neurology 2013;13:11.

* NCT01586702. Intensified secondary prevention intending a reduction of recurrent events in TIA and minor stroke patients
(INSPiRE-TMS). clinicaltrials.gov/ct2/show/NCT01586702 (first received 27 April 2012).

\section{NCT01776034 \{published data only\}}

NCT01776034. Health promotion and wellness program for stroke survivors. clinicaltrials.gov/ct2/show/NCT01776034 (first received 25 January 2013).

Plow M, Moore SM, Kirwan JP, Frost F, Katzan I, Jaeger S. Randomized controlled pilot study of a SystemCHANGE(TM) weight management intervention in stroke survivors: rationale and protocol. Trials 2013;14:130.

NCT01812421 \{published data only\}

NCT01812421. A nested case-control study on the secondary prevention of ischemic stroke and TIA by hypertension health education protocol (HHEP): the post-stroke preventive trial. clinicaltrials.gov/ct2/show/NCT01812421 (first received 18 March 2013).

\section{NCT02132364 \{published data only\}}

NCT02132364. Controlled education of patients after stroke (CEOPS). clinicaltrials.gov/ct2/show/NCT02132364 (first received 7 May 2014).

NCT02140619 \{published data only\}

NCT02140619. Multiple health education interventions for medication compliance and clinical prognosis of ischemic stroke patients. clinicaltrials.gov/ct2/show/NCT02140619 (first received 16 May 2014).

\section{NCT02156778 \{published data only\}}

NCT02156778. Post-stroke disease management - Stroke Card (Stroke Card). clinicaltrials.gov/ct2/show/NCT02156778 (first received 5 June 2014).

* Toell T, Willeit G, Schoenherr C, Tuer A, Pachlaner R, Furtner M. Poststroke disease management - stroke card: extending the standard care after stroke and high-risk TIA. International Journal of Stroke 2014;9(3):284 WSc-0656.

NCT02251834 \{published data only\}

NCT02251834. Hispanic Secondary Stroke Prevention Initiative (HISSPI). clinicaltrials.gov/ct2/show/NCT02251834 (first received 29 September 2014).

\section{NCT02712385 \{published data only\}}

NCT02712385. SPRITE - a feasibility and pilot study (SPRITE). clinicaltrials.gov/ct2/show/NCT02712385 (first received 18 March 2016).

\section{NCT02868723 \{published data only\}}

NCT02868723. Prospective study to optimize the health of patients with TIAs (transient ischemic attacks) and stroke admitted to the Hamad General Hospital (PROMOTE-HEALTH). www.clinicaltrials.gov/ct2/show/NCT02868723 (first received 16 August 2016).

\section{Sarfo 2016 \{published data only\}}

NCT02568137. Phone-based Intervention Under Nurse Guidance After Stroke (PINGS). clinicaltrials.gov/ct2/show/ NCT02568137 (first received 5 October 2015). 
Sarfo F, Treiber F, Jenkins C, Patel S, Gebregziaber M, Singh A, et al. Phone-based intervention under nurse guidance after stroke (PINGS): study protocol for a randomized controlled trial. Trials 2016;17:436.

\section{Spruill 2015 \{published data only\}}

Spruill T, Williams O, Teresi J, Lehrer S, Pezzin L, Waddy S. Comparative effectiveness of home blood pressure monitoring (HBPTM) plus nurse case management versus HBTM alone among black and Hispanic stroke survivors: study protocol for a randomized controlled trial. Trials 2015;16:97.

\section{THRIVES 2013 \{published data only\}}

NCT01900756. Tailored hospital-based risk reduction to impede vascular events after stroke (THRIVES). clinicaltrials.gov/ct2/ show/NCT01900756 (first received 16 July 2013).

* Owolabi MO, Akinyemi RO, Gebregziabher M, Olaniyan O, Salako BL, Arulogun O, et al. Randomized controlled trial of a multipronged intervention to improve blood pressure control among stroke survivors in Nigeria. International Journal of Stroke 2014;9(8):1109-16.

\section{Towfighi 2013 \{published data only\}}

NCT00861081. Intervention to Enable Stroke Survivors in Los Angeles County Hospitals to "Stay Within the Guidelines" (SUSTAIN). clinicaltrials.gov/ct2/show/ NCT00861081 (first received 13 March 2013).

NCT01763203. The SUCCEED trial of secondary stroke prevention. clinicaltrials.gov/show/NCT01763203 (first received 8 January 2013).

Towfighi A, Cheng EM, Sanossian N, Bryg R, Mehta B, Razmara A. Secondary stroke prevention by uniting community and chronic care model teams early to end disparities: the SUCCEED trial. Proceedings of the International Stroke Conference 2015;/:11-13.

Towfighi A, Cheng EM, Sanossian N, Bryg RJ, Mehta B, Hudson L. Secondary stroke prevention by uniting community and chronic care model teams early to end disparities: the SUCCEED trial. Proceedings of the International Stroke Conference 2014 2014;Abstract CT:P49.

\section{Additional references}

\section{Aburto 2013}

Aburto N, Ziolkovska A, Hooper L. Effect of lower sodium intake on health: systematic review and meta-analyses. BMJ 2013;346:f1326.

\section{Albers 2002}

Albers GW, Caplan LR, Easton JD, Fayad PB, Mohr JP, Saver JL, et al. Transient ischaemic attack - proposal for a new definition. New England Journal of Medicine 2002;347(21):1713-6.

\section{Amarenco 2016}

Amarenco P, Lavallée P, Labreuche J, Albers G, Bornstein N, Canhão $P$, et al. One-year risk of stroke after transient ischemic attack or minor stroke. New England Journal of Medicine 2016;374(16):1533-42.

\section{Anderson 1991}

Anderson KM, Odell PM, Wilson PW, Kannel WB. Cardiovascular disease risk profiles. American Heart Journal 1991;1(2):293-8.

\section{Antithrombotic Trialists' Collaboration 2002}

Antithrombotic Trialists' Collaboration. Collaborative metaanalysis of randomised trials of antiplatelet therapy for prevention of death, myocardial infarction, and stroke in high risk patients. BMJ 2002;324(1):71-86.

\section{Barber 2016}

Barber P. Incidence of Transient Ischemic Attack in Auckland, New Zealand, in 2011 to 2012. Stroke 2016;47(9):2183 - 2188.

\section{Bennett 2017}

Bennett D. Association of Physical Activity With Risk of Major Cardiovascular Diseases in Chinese Men and Women. JAMA Cardiology 2017;2(12):1349-1358.

\section{Beswick 1996}

Beswick A, Brindle P. Risk scoring in the assessment of cardiovascular risk.. Current Opinion in Lipidology 2006;17(4):375-86.

\section{British Cardiac Society 1998}

Wood D. Joint British recommendations on prevention of coronary heart disease in clinical practice. Heart 1998;80(Suppl 12):S21-9.

\section{Buckley 2010}

Buckley BS, Byrne MC, Smith SM. Service organisation for the secondary prevention of ischaemic heart disease in primary care. Cochrane Database of Systematic Reviews 2010, Issue 3. Art. No: CD006772. [DOI: 10.1002/14651858.CD006772.pub2]

\section{Canadian Stroke Best Practices 2017}

Lindsay MP, Gubitz G, Bayley M, Hill MD, DaviesSchinkel C, Singh S, et al. Canadian Stroke Best Practices Recommendations (Update 2017). Sixth Edition. Ottawa, Ontario Canada: Heart and Stroke Foundation of Canada, 2017

\section{Cappuccio 2002}

Cappuccio F, Oakeshott P, Strazzullo P, Kerry S. Application of Framingham risk estimates to ethnic minorities in United Kingdom and implications for primary prevention of heart disease in general practice: cross sectional population based study. BMJ 2002;325(7375):1271.

\section{Collins 2016}

Collins R, Reith C, Emberson J, Armitage J, Baigent C, et al. Interpretation of the evidence for the efficacy and safety of statin therapy. Lancet 2016;388(10059):2532-2561.

\section{Craig 2008}

Craig P, Dieppe P, Macintyre S, Michie S, Nazareth I, Petticrew M, et al. Developing and evaluating complex interventions: the new Medical Research Council guidance. BMJ 2008;337:a1655.

\section{Critchley 2012}

Critchley JA, Capewell S. Smoking cessation for the secondary prevention of coronary heart disease. Cochrane Database of 
Systematic Reviews 2012, Issue 2. Art. No: CD003041. [DOI: 10.1002/14651858.CD003041.pub3]

\section{Dawber 1951}

Dawber T, Meadors G, Moore, F. Epidemiological approaches to heart disease: the Framingham Study. American Journal of Public Health 1951;41(3):279-86.

\section{Deeks 2011}

Deeks JD, Higgins JPT, Altman DG on behalf of the Cochrane Statistical Methods Group. Analysing data and undertaking meta-analyses . In: Cochrane Handbook for Systematic Reviews of Complex Interventions Version 5.1.0 [updated March 2011]. Available at www.cochrane-handbook.org/. The Cochrane Collaboration, 2011 (accessed June 2016).

\section{De Schryver 2005}

De Schryver EL, van Gijn J, Kappelle LJ, Koudstaal PJ, Algra A, Dutch TIA trial and SPIRIT study groups. Non-adherence to aspirin or oral anticoagulants in secondary prevention after ischaemic stroke. Journal of Neurology 2005;252(11):1316-21.

\section{Dundas 2001}

Dundas R, Morgan M, Redfern J, Lemic-Stojcevic N, Wolfe C. Ethnic differences in behavioural risk factors for stroke: implications for health promotion. Ethnicity \& Health 2001;6(2):95-103.

\section{Easton 2009}

Easton JD, Saver JL, Albers GW, Alberts MJ, Chaturvedi S, Feldmann E, et al. Definition and evaluation of transient ischemic attack: a scientific statement for healthcare professionals from the American Heart Association/American Stroke Association Stroke Council; Council on Cardiovascular Surgery and Anesthesia; Council on Cardiovascular Radiology and Intervention; Council on Cardiovascular Nursing; and the Interdisciplinary Council on Peripheral Vascular Disease. Stroke 2009;40(6):2276-93.

\section{Emberson 2003}

Emberson J, Whincup P, Morris R, Walker M. Re-assessing the contribution of serum total cholesterol, blood pressure and cigarette smoking to the aetiology of coronary heart disease: impact of regression dilution bias. European Heart Journal 2003;24(19):1719-26.

\section{EPOC 2015}

Effective Practice and Organisation of Care (EPOC). EPOC Taxonomy. Available at: https://epoc.cochrane.org/epoctaxonomy 2015;(1).

\section{ESO 2008}

European Stroke Organisation (ESO) Executive Committee, ESO Writing Committee. Guidelines for management of ischaemic stroke and transient ischaemic attack 2008. Cerebrovascular Diseases 2008;25(5):457-507.

\section{Ettehad 2016}

Ettehad D. Blood pressure lowering for prevention of cardiovascular disease and death: a systematic review and meta-analysis. Lancet 2016;387(10022):957-967.

\section{Fan 2017}

Fan M Yu C, Guo Y, Bian Z, Li X, et al. Effect of total, domainspecific, and intensity-specific physical activity on all-cause and cardiovascular mortality among hypertensive adults in China. Journal of hypertension 2017;36(4):793-800.

\section{Feigin 2014}

Feigin V, Forouzanfar M, Krishnamurthi R, Mensah G, Connor M, Bennett D, et al. Global and regional burden of stroke during 1990-2010: findings from the Global Burden of Disease Study 2010. Lancet 2014;383(9913):245-55.

\section{Feigin 2016}

Feigin V, Roth G, Naghavi M, Parmar P, Krishnamurthi R, Chugh S, et al. Global burden of stroke and risk factors in 188 countries, during 1990-2013: a systematic analysis for the global burden of disease study 2013. Lancet 2016;15(9):913-24.

\section{Fourth SSNAP Annual Report 2016/17}

Royal College of Physicians, Clinical Effectiveness and Evaluation Unit on behalf of the Intercollegiate Stroke Working Party. Royal College of Physicians, Clinical Effectiveness and Evaluation Unit on behalf of the Intercollegiate Stroke Working Party. Rising to the Challenge The Fourth SSNAP Annual Report (2016/17). https://www.strokeaudit.org/Documents/ AnnualReport/2016-17-SSNAP-Annual-Report.aspx (accessed 28 February 2018).

\section{Glader 2010}

Glader EL, Sjolander M, Eriksson M, Lundberg M. Persistent use of secondary preventive drugs declines rapidly during the first 2 years after stroke. Stroke 2010;41(2):397-401.

\section{Goldstein 2008}

Goldstein LB. How much can be gained by more systematic prevention of stroke? International Journal of Stroke 2008;3(4):266-71.

\section{GRADEpro GDT [Computer program]}

McMaster University (developed by Evidence Prime) GRADEpro GDT. Version Last accessed 28/02/2018. Hamilton (ON): McMaster University (developed by Evidence Prime), 2015. Available at gradepro.org.

\section{Hackam 2007}

Hackam DG, Spence JD. Combining multiple approaches for the secondary prevention of vascular events after stroke: a quantitative modelling study. Stroke 2007;38(6):1881-5.

\section{Hankey 2014}

Hankey G. Secondary stroke prevention. Lancet Neurology 2014;13(2):178-94.

\section{He 2004}

He K, Song Y, Daviglus ML, Liu K, Van Horn L, Dyer AR, et al. Fish consumption and incidence of stroke: a meta-analysis of cohort studies. Stroke 2004;35(7):1538-42. 


\section{He 2006}

He FJ, Nowson A, MacGregor GA. Fruit and vegetable consumption and stroke: meta-analysis of cohort studies. Lancet 2006;367(9507):320-6.

\section{He 2013}

He FJ, Li J, MacGregor GA. Effect of longer-term modest salt reduction on blood pressure. Cochrane Database of Systematic Reviews 2013, Issue 4. Art. No: CD004937. [DOI: 10.1002/14651858.CD004937.pub2]

\section{Health Foundation 2014}

Health Foundation. Person-centred care made simple: what everyone should know about personcentred care. www.health.org.uk/sites/health/files/ PersonCentredCareMadeSimple.pdf (accessed prior to 19 February 2018).

\section{Herttua 2016}

Herttua k, Martikainen P, Batty D, Kivimäki M. Statin and Antihypertensive Therapies as Risk Factors for Fatal Stroke. Jounral of the American College of Cardiology 2016;67(13):1507-151.

\section{Higgins 2002}

Higgins JPT, Thompson SG. Quantifying heterogeneity in a meta-analysis. Statistics in Medicine 2002;21(11):1539-58.

\section{Higgins 2003}

Higgins JPT, Thompson SG, Deeks JJ, Altman DG. Measuring inconsistency in meta-analyses. BMJ 2003;327:557-60.

\section{Higgins 2011a}

Higgins JPT, Altman DG. Chapter 8: Assessing risk of bias in included studies. In: Higgins JPT, Green S, editors(s). Cochrane Handbook for Systematic Reviews of Interventions Version 5.1.0 [updated March 2011]. Available at www.cochranehandbook.org/. The Cochrane Collaboration, 2011 (accessed 16 November 2012).

\section{Higgins 2011b}

Higgins JPT, Deeks JJ, Douglas AG. Chapter 16: Special topics in statistics. In: Higgins JPT, Green S, editors(s). Cochrane Handbook for Systematic Reviews of Interventions Version 5.1.0 [updated March 2011]. Available at www.cochranehandbook.org/. The Cochrane Collaboration, 2011 (accessed June 2016).

\section{Higgins 2011c}

Higgins JPT, Green S (editors). Cochrane Handbook for Systematic Reviews of Interventions Version 5.1.0 [updated March 2011]. The Cochrane Collaboration, 2011. training.cochrane.org/handbook (accessed pror to 19 February 2018).

\section{Holmes 2014}

Holmes M, Dale C, et al. Association between alcohol and cardiovascular disease: Mendelian randomisation analysis based on individual participant data. BMJ 2014;349:g4164.

\section{Horne 2006}

Horne R, Weinman J, Barber N, Elliot RA, Morgan M. Concordance, adherence and compliance in medication taking: a conceptual map and research priorities. Report for the National Co-ordinating Centre for NHS Service Delivery and Organisation R \& D (NCCSDO) December 2005. www.netscc.ac.uk/hsdr/files/project/ SDO_FR_08-1412-076_V01.pdf (accessed prior to 19 February 2018).

\section{Institute of Public Care 2013}

Institute of Public Care and Centre for Workforce Intelligence. Integrated care: promoting better workforce decisions. http:// www.skillsforcare.org.uk/Document-library/NMDS-SC, workforce-intelligence-and-innovation/Research/IPC-ER4--Integrated-Health-and-Social-Care-Report-100613-FINAL.pdf 2013.

\section{Kernan 2014}

Kernan W, Ovbiagele B, Black H, Bravata D, Chimowitz M, et al. Guidelines for the Prevention of Stroke in Patients With Stroke and Transient Ischemic AttackA Guideline for Healthcare Professionals From the American Heart Association/American Stroke Association. Stroke 2014;45(7):2160-236.

\section{Kernan 2016}

Kernan W, Viscoli C, Furie K, Young S, Inzucchi S, Gorman M, the IRIS Trial Investigators. Pioglitazone after ischemic stroke or transient ischemic attack. New England Journal of Medicine 2016;374(14):1321-31

\section{Lager 2012}

Lager K, Wilson A, Khunti K, Mistri AK. Quality of secondary prevention measures in TIA patients: a retrospective cohort study. Postgraduate Medical Journal 2012;88(1040):305-11.

\section{Lemieux-Charles 2006}

Lemieux-Charles L, McGuire W. What do we know about health care team effectiveness? A review of the literature. Medical Care Research and Review 2006;63(3):263-300.

\section{Lindson-Hawley 2015}

Lindson-Hawley N, Thompson T, Begh R. Motivational interviewing for smoking cessation. Cochrane Database of Systematic Reviews 2015, Issue 3. Art. No: CD006936. [DOI: 10.1002/14651858.CD006936.pub2]

\section{Logue 2015}

Longue J. Systematic review of studies exploring reasons for statin non-adherence an of randomised controlled trials of interventions to improve adherence. Atherosclerosis 2015;241:E52-E52.

\section{MacKay-Lyons 2013}

MacKay-Lyons M, Thornton M, Ruggles T, Manley S. Nonpharmacological interventions for preventing secondary vascular events after stroke or transient ischemic attack. Cochrane Database of Systematic Reviews 2013, Issue 3. Art. No: CD008656. [DOI: 10.1002/14651858.CD008656.pub2] 


\section{NAO 2005}

National Audit Office. Department of Health. Reducing brain damage: faster access to better stroke care (2005). www.nao.org.uk/publications/0506/ reducing_brain_damage.aspx (accessed 16 November 2012).

\section{National Stroke Foundation 2017}

Stroke Foundation Melbourne Australia. Clinical Guidelines for Stroke Management 2017. Clinical Guidelines for Stroke Management 2017 (https://strokefoundation.org.au/What-wedo/Treatment-programs/Clinical-guidelines) 2017.

\section{NHS 2014}

NHS Employers and General Practitioners Committee. Quality and outcomes framework guidance for GMS contract 2014/15. www.nhsemployers.org/-/media/Employers/Documents/ Primary-care-contracts/QOF/2014-15/2014-15-General-MedicalServices-contract---Quality-and-Outcomes-Framework.pdf? la=en\&hash $=69$ CF3870EB19476ECDFF3A5CBAA35F08C34E00DC 2014.

\section{O'Donnell 2010}

O'Donnell MJ, Xavier D, Liu L, Zhang H, Siu LC, Purnima R, et al. Risk factors for ischaemic and intracerebral haemorrhagic stroke in 22 countries (the INTERSTROKE study): a case-control study. Lancet 2010;376(9735):112-23.

\section{Ovbiagele 2004}

Ovbiagele B, Saver JL, Fredieu A, Suzuki S, McNair N, Dandekar A, et al. PROTECT: a coordinated stroke treatment program to prevent recurrent thromboembolic events. Neurology 2004;63(7):1217-22.

\section{Perk 2012}

Perk 2012. European Guidelines on cardiovascular disease prevention in clinical practice (version 2012). The Fifth Joint Task Force of the European Society of Cardiology and Other Societies on Cardiovascular Disease Prevention in Clinical Practice (constituted by representatives of nine societies and by invited experts). European Heart Journal 2012;33(13):1635-1701.

\section{Perreault 2012}

Perreault S, Yu A, Côté R. Adherence to antihypertensive agents after ischemic stroke and risk of cardiovascular outcomes. Neurology 2012;79(20):2037-40.

\section{Preiss 2015}

Preiss D, Kristensen S. The new pooled cohort equations risk calculator. Canadian journal of Cardiology 2015;31(5):613-619.

\section{Raine 2009}

Raine R, Wong W, Ambler G, Hardoon S, Petersen I, Morris R, et al. Sociodemographic variations in the contribution of secondary drug prevention to stroke survival at middle and older ages: cohort study. BMJ 2009;338:b1279.

\section{Ramsay 2007}

Ramsay SE, Whincup PH, Wannamethee SG, Papacosta O, Lennon L, Thomas MC, et al. Missed opportunities for secondary prevention of cerebrovascular disease in elderly British men from 1999 to 2005: a population-based study. Journal of Public Health 2007;29(3):251-7.

\section{RCP 2016}

Intercollegiate Stroke Working Party. Royal College of Physicians. https://www.strokeaudit.org/SupportFiles/ Documents/Guidelines/2016-National-Clinical-Guideline-forStroke-5t-(1).aspx 2016.

\section{Redfern 2008}

Redfern J, Rudd AD, Wolfe CD, McKevitt C. Stop Stroke: development of an innovative intervention to improve risk factor management after stroke. Patient Education \& Counseling 2008;72(2):201-9.

\section{RevMan 2014 [Computer program]}

The Nordic Cochrane Centre, The Cochrane Collaboration Review Manager (RevMan). Version 5.3. Copenhagen: The Nordic Cochrane Centre, The Cochrane Collaboration, 2014.

\section{Reynolds 2003}

Reynolds K, Lewis LB, Nolen JDL, Kinney GL, Sathya B, He J. Alcohol consumption and risk of stroke: a meta-analysis. JAMA 2003;289(5):579-88.

\section{Rice 2017}

Rice VH, Heath L, Livingstone-Banks J, Hartmann-Boyce J. Nursing interventions for smoking cessation. Cochrane Database of Systematic Reviews 2017, Issue 12. Art. No: CD001188. [DOI: 10.1002/14651858.CD001188.pub5]

\section{Rose 1981}

Rose G. Strategy of prevention: lessons from cardiovascular disease. BMJ (Clinical Research Ed.) 1981;282(6279):1847-51.

\section{Rudd 2004}

Rudd AG, Lowe D, Hoffman A, Irwin P, Pearson M. Secondary prevention for stroke in the United Kingdom: results from the National Sentinel Audit of Stroke. Age \& Ageing 2004;33(3):280-6.

\section{Saunders 2016}

Saunders DH, Sanderson M, Hayes S, Kilrane M, Greig CA, Brazzelli M, et al. Physical fitness training for stroke patients. Cochrane Database of Systematic Reviews 2016, Issue 3. Art. No: CD003316. [DOI: 10.1002/14651858.CD003316.pub6]

\section{SIGN 2008}

Scottish Intercollegiate Guidelines Network. SIGN 108: Management of patients with stroke: assessment, investigation, immediate management and secondary prevention 2008). http://www.sign.ac.uk/sign-108-management-of-patientswith-stroke-or-tia-assessment-investigation-immediatemanagement-a.html (accessed prior to 19 February 2018).

\section{Starfield 2002}

Starfield B, Shi L. Policy relevant determinants of health: an international perspective. Health Policy 2002;60(3):201-18. 


\section{Stead 2013a}

Stead LF, Hartmann-Boyce J, Perera R, Lancaster T. Telephone counselling for smoking cessation. Cochrane Database of Systematic Reviews 2013, Issue 8. Art. No: CD002850. [DOI: 10.1002/14651858.CD002850.pub3]

\section{Stead 2013b}

Stead LF, Buitrago D, Preciado N, Sanchez G, HartmannBoyce J, Lancaster T. Physician advice for smoking cessation. Cochrane Database of Systematic Reviews 2013, Issue 5. Art. No: CD000165. [DOI: 10.1002/14651858.CD000165.pub4]

\section{Stead 2017}

Stead F, Carroll J, Lancaster T. Group behaviour therapy programmes for smoking cessation. Cochrane Database of Systematic Reviews 2017, Issue 3. Art. No: CD001007. [DOI: 10.1002/14651858.CD001007.pub3]

\section{Stroke Association 2018}

Stroke Association. State of the nation: stroke statistics January 2018. https://www.stroke.org.uk/system/files/sotn_2018.pdf (accessed 28 February 2018).

\section{Stroke Audit 2016}

Stroke Audit 2016. www.strokeaudit.org/SupportFiles/ Documents/Guidelines/2016-National-Clinical-Guideline-forStroke-5t-(1).aspx (accessed 9 October 2017).

\section{Stroke Unit Trialists' Collaboration 2013}

Stroke Unit Trialists' Collaboration. Organised inpatient (stroke unit) care for stroke. Cochrane Database of Systematic Reviews 2013, Issue 9. Art. No: CD000197. [DOI: 10.1002/14651858.CD000197.pub3]

\section{Sundström 2014}

Sundström J. Blood pressure lowering and cardiovascular risk Authors' reply. Lancet 2014;384(9956):1746-1747.

\section{Swientozielskyj 2015}

Swientozielskyj S, Nwosu A, Cooper A, Bryant E, Baggaley G, Murgatroyd $\mathrm{H}$, et al. NHS England. MDT development - working toward an effective multidisciplinary/multi agency team. www.england.nhs.uk/wp-content/uploads/2015/01/mdt-devguid-flat-fin.pdf (accessed prior to 19 February 2018).

\section{Taylor 2017}

Taylor G, Dalili M, Semwal M, Civljak M, Sheikh A, Car J. Internetbased interventions for smoking cessation. Cochrane Database of Systematic Reviews 2017, Issue 9. Art. No: CD007078. [DOI: 10.1002/14651858.CD007078]

\section{Touze 2005}

Touze E, Varenne O, Chatellier G, Peyrard S, Rothwell PM, Mas JL. Risk of myocardial infarction and vascular death after transient ischemic attack and ischemic stroke: a systematic review and meta-analysis. Stroke 2005;36(12):2748-55.

\section{Van Wijk 2005}

Van Wijk I, Kappelle LJ, Van Gijn J, Koudstaal PJ, Franke CL, Vermeulen $\mathrm{M}$, et al. Long-term survival and vascular event risk after transient ischaemic attack or minor ischaemic stroke: a cohort study. Lancet 2005;365:2098-104.

\section{Wensing 2006}

Wensing M, Wollersheim H, Grol R. Organizational interventions to implement improvements in patient care: a structured review of reviews. Implementation Science 2006;1(1):2.

\section{Whittaker 2016}

Whittaker R, McRobbie H, Bullen C, Rodgers A, Gu Y. Mobile phone-based interventions for smoking cessation. Cochrane Database of Systematic Reviews 2016, Issue 4. Art. No: CD006611. [DOI: 10.1002/14651858.CD006611.pub3]

\section{WHO 1978}

World Health Organization. Cerebrovascular disorders: a clinical and research classification. 1978. apps.who.int/iris/ handle/10665/37194 (accessed prior to 19 February 2018).

\section{WHO 2008}

World Health Organization. The World Health Report 2008: primary health care now more than ever. www.searo.who.int/ LinkFiles/Reports_whr08_en.pdf (accessed 16 November 2012).

\section{WHO 2017}

World Health Organisation. Cardiovascular diseases. http:// www.who.int/mediacentre/factsheets/fs317/en/ 2017.

\section{Wilson 1998}

Wilson PW, D'Agostino RB, Levy D, Belanger AM, Silbershatz H, Kannel WB. Prediction of coronary heart disease using risk factor categories. Circulation 1998;97(18):1837-47.

\section{Xu 2017}

Xu T, Yu X, Ou S, Liu X, Yuan J, Tan X, Chen Y. Adherence to Antihypertensive Medications and Stroke Risk: A Dose Response Meta-Analysis. Journal of the American Heart Association 2017;6(7):e006371.

\section{References to other published versions of this review}

\section{Lager 2011}

Lager KE, Wilson AD, Mistri AK, Khunti K. Stroke services for risk reduction in the secondary prevention of stroke. Cochrane Database of Systematic Reviews 2011, Issue 6. Art. No: CD009103. [DOI: 10.1002/14651858.CD009103]

\section{Lager 2014}

Lager KE, Mistri AK, Khunti K, Haunton VJ, Sett AK, Wilson AD. Interventions for improving modifiable risk factor control in the secondary prevention of stroke. Cochrane Database of Systematic Reviews 2014, Issue 5. Art. No: CD009103. [DOI: 10.1002/14651858.CD009103.pub2]

* Indicates the major publication for the study 
CHARACTERISTICS OF STUDIES

Characteristics of included studies [ordered by study ID]

Adie 2010

\section{Study characteristics}

\begin{tabular}{ll}
\hline Methods & RCT \\
& Unit of randomisation: participant
\end{tabular}

Participants

Place of recruitment: hospital stroke clinic and hospital neurovascular clinic

Numbers randomised: total: 56; (I: 29; C: 27$)$

$\%$ Completing final follow-up: $100 \%$

Inclusion criteria: < 1 month since minor stroke or TIA; > 18 years; clinic SBP $\geq 140$ mmHg; living at home at time of follow-up

Exclusion criteria: known dementia, "significant disability or co-morbidity which would impair ability to consent or cause undue distress"

Type of stroke: minor stroke (57\%); TIA (43\%)

Mean age (SD): 72.5 (8.9)

Gender (\% men): 50\%

Ethnicity: not reported

Socio-economic or socio-demographic status: not reported

Interventions

Intervention details (components, length, frequency): motivational telephone follow-up intervention based on social cognitive theory. Participants received a 20 minute telephone call at 7 days, 1, 2 and 4 months to review risk factors, medication and goal setting; participants provided with tailored educational material; participants with high blood pressure encouraged to visit their GP

Location: community

Mode of delivery: telephone follow-up

Personnel responsible for delivery: 1 researcher

Timing post-stroke: $<1$ month

Control: usual care (participants received instructions for follow-up with their GP; no follow-up visits arranged in secondary care)

Outcomes

6 months: SBP (clinic and ambulatory); DBP (clinic and ambulatory); total cholesterol; BP $\leq 130 / 80$ $\mathrm{mmHg}$; total cholesterol $\leq 4 \mathrm{mmol} / \mathrm{L}$

Country of origin: UK

Publication language: English

Notes Analysis method: not stated

Risk of bias: low

Comments: definition of minor stroke not stated 
Adie 2010 (Continued)

Risk of bias

\begin{tabular}{|c|c|c|}
\hline Bias & Authors' judgement & Support for judgement \\
\hline $\begin{array}{l}\text { Random sequence genera- } \\
\text { tion (selection bias) }\end{array}$ & Low risk & $\begin{array}{l}\text { Envelope method: "participants were randomized ... at the end of their first } \\
\text { study visit (baseline; month 0) by sequential opaque envelopes stratified by } \\
\text { stroke or TIA" }\end{array}$ \\
\hline $\begin{array}{l}\text { Allocation concealment } \\
\text { (selection bias) }\end{array}$ & Low risk & Envelope method \\
\hline $\begin{array}{l}\text { Incomplete outcome data } \\
\text { (attrition bias) } \\
\text { All outcomes }\end{array}$ & Low risk & No missing outcome data \\
\hline $\begin{array}{l}\text { Selective reporting (re- } \\
\text { porting bias) }\end{array}$ & Low risk & Protocol available and outcomes are reported in the pre-specified way \\
\hline Other bias & Low risk & The study appears to be free of other sources of bias \\
\hline
\end{tabular}

Allen 2002

\section{Study characteristics}

\begin{tabular}{|c|c|}
\hline Methods & $\begin{array}{l}\text { RCT } \\
\text { Unit of randomisation: participant }\end{array}$ \\
\hline Participants & $\begin{array}{l}\text { Place of recruitment: hospital acute stroke department } \\
\text { Numbers randomised: total: } 96 \text { (I: 47; C: 46) } \\
\% \text { Completing final follow-up: } 76 \% \\
\text { Inclusion criteria: ischaemic stroke or TIA; discharged to home or short-term rehabilitation facility (for < } \\
1 \text { month); no other illnesses that would dominate post-discharge care; Rankin Scale score } 3 \text {; } \\
\text { Exclusion criteria: Rankin score of } 4 \text { or 5; discharged to long-term care facility } \\
\text { Type of stroke: ischaemic stroke (I: } 70 \% ; C: 71 \%) ; \text { TIA (I: } 30 \% ; C: 29 \%) \\
\text { Mean age (SE): I: } 69 \text { (1.7); C: } 72 \text { (1.5) } \\
\text { Gender (\% women): I: } 57 ; \text { C: } 54 \\
\text { Ethnicity (\% African-American): I: } 30 \% \text {; : } 20 \% \\
\text { Socio-economic or socio-demographic status: not reported }\end{array}$ \\
\hline Interventions & $\begin{array}{l}\text { Intervention details (components, length, frequency): APN telephoned patients } 3 \text { to } 7 \text { days post-dis- } \\
\text { charge to assess needs and deliver education; APN conducted home assessment within } 1 \text { month post- } \\
\text { discharge; individualised patient care plans developed by interdisciplinary team using evidence-based } \\
\text { recommendations; APN implemented treatment plan and conducted follow-up assessments; primary } \\
\text { care physicians provided with care plans/evidence-based recommendations } \\
\text { Location: community } \\
\text { Mode of delivery: home visits }\end{array}$ \\
\hline
\end{tabular}


Allen 2002 (Continued)

Personnel responsible for delivery: advanced practice nurse and interdisciplinary team

Timing post-stroke: discharge home

Control: usual care provided by primary care physician

Pre-discharge care (I and C): interdisciplinary care and stroke education

\begin{tabular}{ll}
\hline Outcomes & 3 months: BP: mean $\mathrm{mmHg} \mathrm{BP}>140 / 90$; proportion of participants re-hospitalised for stroke \\
\hline General Information & Funding: not reported \\
& Country of origin: USA \\
& Publication language: English \\
\hline Notes & Analysis method: not stated \\
& Risk of bias: unclear \\
\hline
\end{tabular}

\section{Risk of bias}

Bias Authors' judgement Support for judgement

Random sequence genera- Low risk tion (selection bias)

"Patients were assigned to the intervention or to usual postdischarge care by drawing consecutive concealed tickets that were randomized within permuted blocks of $10 "$

\begin{tabular}{ll}
\hline $\begin{array}{l}\text { Allocation concealment } \\
\text { (selection bias) }\end{array}$ & Unclear risk \\
\end{tabular}

\begin{tabular}{|c|c|c|}
\hline \multirow{3}{*}{$\begin{array}{l}\text { Incomplete outcome data } \\
\text { (attrition bias) } \\
\text { All outcomes }\end{array}$} & \multirow[t]{3}{*}{ Unclear risk } & Missing data not reported by group \\
\hline & & $\begin{array}{l}\text { Attrition: } 1 \text { became cognitively impaired; } 2 \text { moved out of state; } 3 \text { moved to } \\
\text { nursing home; } 5 \text { died; } 12 \text { refused follow up visit }\end{array}$ \\
\hline & & $\begin{array}{l}\text { Judgement: not enough information to permit judgement (missing data not } \\
\text { reported by group) }\end{array}$ \\
\hline
\end{tabular}

\begin{tabular}{lll}
\hline $\begin{array}{l}\text { Selective reporting (re- } \\
\text { porting bias) }\end{array}$ & Unclear risk & Insufficient information (protocol not obtained) \\
\hline Other bias & Low risk & The study appears to be free of other sources of bias \\
\hline
\end{tabular}

\section{Allen 2009}

\section{Study characteristics}

\begin{tabular}{ll}
\hline Methods & RCT \\
& Unit of randomisation: participant \\
\hline Participants & Place of recruitment: hospital acute stroke department \\
& Numbers randomised: total: 380 (I: $190 ;$ C: 190$)$ \\
& $\%$ Completing final follow-up: $84 \%$ to $100 \%$ depending on outcome measure
\end{tabular}


Allen 2009 (Continued)

Inclusion criteria: ischaemic stroke; NIHSS $\geq 1$; discharged to home or short-term rehabilitation/nursing facility (for < 8 weeks); no other illnesses that would dominate post-discharge care; English-speaking; no planned carotid endarterectomy

Type of stroke: ischaemic (100\%)

Mean age (SE): I: 68 (1); C: 69 (1)

Gender (\% men): I: 48\%; C: 52\%

Ethnicity (\% African American): I: 17\%; C: 15\%

Socio-economic or socio-demographic status (\% married): I: 47\%; C: 46\%

Interventions

Intervention details: participant received home assessment at 1 week from APN; individualised patient care plans developed by interdisciplinary team using evidence-based recommendations; ongoing care management provided by APN for 6 months (telephone contact every week for first month and monthly thereafter; home visits as needed; physical therapist visits arranged as needed; liaison with social services; participants provided with personalised health record and pill organisers for risk factor management); primary care physicians provided with care plans/evidence-based recommendations

Location: community

Mode of delivery: home visits and telephone follow-up

Personnel responsible for delivery: APN and interdisciplinary team

Timing post-stroke: discharge home

Control: usual care provided by primary care physician; received postal stroke-related educational materials every 2 months

Usual care before discharge (I and C): organised stroke department care with enhanced discharge planning. Involved physical and psychological evaluation using standardised assessment tools; initiation of appropriate medication; development of individualised discharge plan; discharge summary sent to primary care physician

Outcomes

6 months: SBP > $140 \mathrm{mmHg}$; DBP > 90 mmHg; total cholesterol > $180 \mathrm{mg} / \mathrm{dL}$; $\mathrm{Hb} 1 \mathrm{Ac}>6.5 \%$; proportion of participants on anticoagulant; proportion of participants using method for medication compliance

$\begin{array}{ll}\text { General Information } & \text { Funding: not reported } \\ \text { Country of origin: USA } \\ \text { Publication language: English }\end{array}$

Notes

Analysis method: stated intention-to-treat

Risk of bias: unclear

\section{Risk of bias}

\begin{tabular}{lll}
\hline Bias & Authors' judgement & Support for judgement \\
\hline $\begin{array}{l}\text { Random sequence genera- } \\
\text { tion (selection bias) }\end{array}$ & Low risk & $\begin{array}{l}\text { "The randomization sequence was by permuted blocks of fixed size (10) gener- } \\
\text { ated by study biostatisticians" }\end{array}$ \\
\hline $\begin{array}{l}\text { Allocation concealment } \\
\text { (selection bias) }\end{array}$ & Low risk & $\begin{array}{l}\text { "Group assignment was made by a research assistant using the sealed enve- } \\
\text { lope method" }\end{array}$ \\
\hline
\end{tabular}

Incomplete outcome data Unclear risk Missing data reported by group but reasons not fully described
(attrition bias)

All outcomes

Interventions for improving modifiable risk factor control in the secondary prevention of stroke (Review) 
Allen 2009 (Continued)

Attrition (dependent on outcome): I: range 0/90 to 25/190 (reasons unclear); C: range $0 / 190$ to $36 / 190$ (reasons unclear)

Judgement: not enough information to permit judgement (reasons for missing data not provided)

$\begin{array}{ll}\begin{array}{l}\text { Selective reporting (re- } \\ \text { porting bias) }\end{array} & \text { Low risk }\end{array}$

Other bias Low risk The study appears to be free of other sources of bias

Boter 2004

\section{Study characteristics}

Methods RCT

Unit of randomisation: participant

Participants

Place of recruitment: 2 university hospitals; 10 general hospitals

Numbers randomised: total: 536 (I: 263; C: 273)

$\%$ Completing final follow-up: $91 \%$

Inclusion criteria: TIA, ischaemic stroke, primary intracerebral haemorrhage, or subarachnoid haemorrhage; Dutch-speaking; $\geq 18$ years; first admission for stroke or TIA; hospitalisation within 72 hours after onset of symptoms; life expectancy > 1 year; Rankin grade 0 to 3; discharged home

Type of stroke: TIA (I: 9\%; C: 8\%); ischaemic stroke (I: 53\%; C: 55\%); haemorrhagic stroke (I: 10\%; C: 9\%); subarachnoid haemorrhage (I: 19\%; C: 19\%)

Median age (IQR): I: 66 (52 to 76$)$; C: 63 (51 to 74 )

Gender (\% women): I: 51\%; C: 52\%

Ethnicity (\% African American): I: 17\%; C: $15 \%$

Socio-economic or socio-demographic status:

- Education level: I: primary school or less - $24 \%$, secondary school-60\%, higher education or university - 15 , unknown - $1 \%$; C: primary school or less - $27 \%$, secondary school - $58 \%$, higher education or university - $15 \%$, unknown $<1 \%$

- Living alone: I: $30 \%$, C: $26 \%$

Interventions

Intervention details (components, length, frequency): participants and their carers received 3 telephone calls from a stroke nurse at 1 to 4,4 to 8 and 18 to 24 weeks; participants received 1 home visit from a stroke nurse at 10 to 14 weeks; checklists used to address stroke risk factors, stroke consequences and unmet needs in terms of stroke services; nurses supported participants and carers according to their individual needs

Location: community

Mode of delivery: home visits and telephone follow-up

Personnel responsible for delivery: stroke nurses trained for 2 days on "secondary prevention of stroke, rehabilitation, therapies, prognosis and knowledge of local care facilities"

Timing post-stroke: post-discharge 
Boter 2004 (Continued)

Control: standard care

Outcomes 6 months: proportion of participants using secondary prevention drugs (anticoagulants or antiplatelets)

General Information $\quad$ Funding: clinical investigator grant from the Netherlands Heart Foundation (grant D98.014), by a grant from the Netherlands Heart Foundation and the Netherlands Organization for Health Research and Development (940-32014), and by a grant from the University Medical Center Utrecht

Country of origin: Netherlands

Publication language: English

\begin{tabular}{ll}
\hline Notes & Analysis method: stated intention-to-treat \\
& Risk of bias: low \\
\hline
\end{tabular}

\section{Risk of bias}

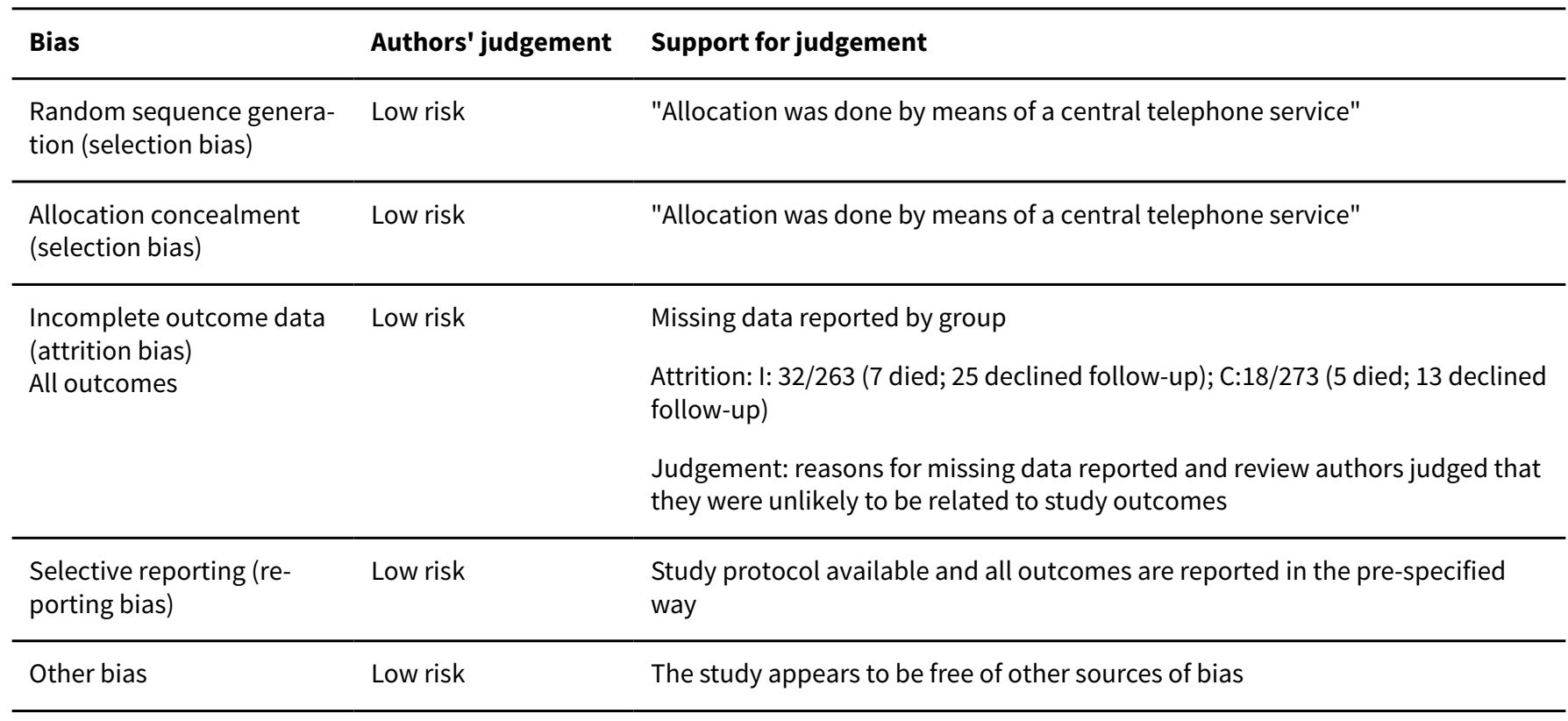

Boysen 2009

\section{Study characteristics}

Methods RCT

Unit of randomisation: participant

Participants
Numbers randomised: total: 314 (I: 157; C: 157$)$
\% Completing final follow-up: $88 \%$
Inclusion criteria: ischaemic stroke; aged > 40 years; able to walk
Exclusion criteria: contraindications to exercise; modified Rankin scale of 4 or 5 pre-stroke; cognitive
impairment; discharge to nursing home; severe neurological deficit


Boysen 2009 (Continued)

Type of stroke: ischaemic (100\%)

Median age (IQR): I: 69.7 (60.0 to 77.7); C: 69.4 (59.6 to 75.8)

Gender (\% women): I: 43\%; C: 44\%

Ethnicity: not reported

Socio-economic or socio-demographic status:

- Years of education (\%): I: $\leq 8$ (45\%), 9 to $12(34 \%), \geq 13(21 \%)$; C: $\leq 8(47 \%), 9$ to $12(40 \%), \geq 13(13 \%)$

Interventions

Intervention details (components, length, frequency): repeated verbal instructions about physical activity over 2 years; first meeting (30 to 60 minutes) to develop individualised plan for physical activity; follow-up visits ( 20 to 30 minutes) every 3 months for the first year and every 6 months thereafter to provide repeated instructions and readjust physical activity plan; between-visit reminder telephone calls

Location: community

Mode of delivery: home visits and telephone follow-up

Personnel responsible for delivery: physiotherapist in 8 centres, neurologist in 1 centre

Timing post-stroke: beginning $<90$ days post-stroke

Control: received information about physical activity; received follow-up visits at same frequency as intervention group but without instructions about physical activity

\begin{tabular}{|c|c|}
\hline Outcomes & 24 months: number of secondary strokes; number of myocardial infarctions; number of vascular deaths \\
\hline \multirow[t]{3}{*}{ General Information } & $\begin{array}{l}\text { Funding: the Ex Stroke Pilot Trial was funded by the Ludvig and Sara Elsass' Foundation, Hede Nielsen } \\
\text { Foundation, Eva and Henry Frænkel's Foundation, Søren and Helene Hempel's Foundation, and King } \\
\text { Christian X Foundation }\end{array}$ \\
\hline & Country of origin: Denmark, China, Poland and Estonia \\
\hline & Publication language: English \\
\hline \multirow[t]{2}{*}{ Notes } & Analysis method: stated intention-to-treat; per protocol \\
\hline & Risk of bias: low \\
\hline
\end{tabular}

\section{Risk of bias}

\begin{tabular}{|c|c|c|}
\hline Bias & Authors' judgement & Support for judgement \\
\hline $\begin{array}{l}\text { Random sequence genera- } \\
\text { tion (selection bias) }\end{array}$ & Low risk & $\begin{array}{l}\text { Central randomisation: "generation of allocation sequences was computer } \\
\text { based" }\end{array}$ \\
\hline $\begin{array}{l}\text { Allocation concealment } \\
\text { (selection bias) }\end{array}$ & Low risk & $\begin{array}{l}\text { "Allocation concealment was achieved through centralised randomization by } \\
\text { telephone or email." }\end{array}$ \\
\hline \multirow{3}{*}{$\begin{array}{l}\text { Incomplete outcome data } \\
\text { (attrition bias) } \\
\text { All outcomes }\end{array}$} & Low risk & Missing data reported by group \\
\hline & & $\begin{array}{l}\text { Attrition: I: } 24 / 157 \text { ( } 11 \text { died; } 3 \text { withdrawn due to severe neurological deficits } \\
\text { caused by recurrent stroke; } 10 \text { lost to follow-up); C: } 14 / 157 \text { ( } 9 \text { died; } 2 \text { with- } \\
\text { drawn due to severe neurological deficits caused by recurrent stroke; } 2 \text { lost to } \\
\text { follow-up) }\end{array}$ \\
\hline & & $\begin{array}{l}\text { Judgement: reasons for missing data reported and review authors judge that } \\
\text { they are unlikely to be related to study outcomes }\end{array}$ \\
\hline
\end{tabular}


Boysen 2009 (Continued)

Selective reporting (re- Low risk Study protocol available and all outcomes are reported in the pre-specified porting bias) way

Other bias

Low risk

The study appears to be free of other sources of bias

Brotons 2011

\section{Study characteristics}

Methods RCT

Unit of randomisation: general practice

Place of recruitment: 42 primary care centres in 8 regions of Spain
Numbers randomised: total: 1224 (414 stroke/TIA); I: 624 (203 stroke/TIA); C: 600 (211 stroke/TIA)
\% Completing final follow-up: $70 \%$
Inclusion criteria: cardiovascular disease (ischaemic heart disease, stroke /TIA and peripheral arterial
disease); 80 years
Exclusion criteria: cardio-embolic stroke or subarachnoid haemorrhage as a result of valvulopathy; se-
rious disease or terminal illness; bed bound
Type of stroke (\%): not stated
Mean age (SE): I: 68 (11); C: 69 (11)
Gender (\% men): I: $64 \% ;$ C: $64 \%$
Ethnicity: not reported
Socio-economic or socio-demographic status:
- Employment status: employed - 11\%, unemployed - 2\%, sick leave/invalidity - 10\%, retired 61\%, Other
- $16 \%$
Education level: illiterate - 4\%, uneducated, literate - 36\%, primary education - 39\%, secondary edu-
cation - 13\%, higher education - 6\%, university 3\%

Interventions

Intervention details (components, length, frequency): comprehensive secondary prevention program including tailored patient education, promotion of medication adherence and review of secondary prevention medication; participants attended appointment every 4 months for 2.75 years; health professionals delivering the intervention followed protocols for patient care and attended training sessions on secondary prevention of cardiovascular disease

Location: primary care

Mode of delivery: outpatient appointment

Personnel responsible for delivery: nurses with specific training in the secondary prevention of cardiovascular disease

Timing post-stroke: $<1$ year

Control: usual care

Outcomes

3 years: SBP; DBP; total cholesterol; LDL; HDL; triglycerides; BMI; BP $<140 / 90$ in non-diabetics or BP < $130 / 80$ in diabetics/ patients with chronic renal failure; cardiovascular readmissions; cardiovascular fatal events 
Brotons 2011 (Continued)

General Information
Funding: project co-ordinated and funded by the FIS (PI031421), Instituto de Salud Carlos III, Ministry of Health and Consumer Affairs

Country of origin: Spain

Publication language: English

Notes Analysis method: intention-to-treat

Risk of bias: low

\section{Risk of bias}

\begin{tabular}{|c|c|c|}
\hline Bias & Authors' judgement & Support for judgement \\
\hline $\begin{array}{l}\text { Random sequence genera- } \\
\text { tion (selection bias) }\end{array}$ & Low risk & Random numbers generated using a validated computer program \\
\hline $\begin{array}{l}\text { Allocation concealment } \\
\text { (selection bias) }\end{array}$ & Low risk & $\begin{array}{l}\text { Central allocation service, stratified by region ("the randomization sequence } \\
\text { was not revealed until the intervention was assigned") }\end{array}$ \\
\hline \multirow{4}{*}{$\begin{array}{l}\text { Incomplete outcome data } \\
\text { (attrition bias) } \\
\text { All outcomes }\end{array}$} & Low risk & Missing data reported by group \\
\hline & & $\begin{array}{l}\text { Attrition: I: } 11 \text { died; } 51 \text { lost to follow-up (reasons provided); } 6 \text { unknown; C: } 13 \\
\text { died; } 69 \text { lost to follow-up (reasons provided); } 41 \text { unknown* }\end{array}$ \\
\hline & & $\begin{array}{l}\text { *study authors explain that it was difficult to recover reasons for losses in con- } \\
\text { trol group because they were visited only at baseline and at end of follow-up }\end{array}$ \\
\hline & & $\begin{array}{l}\text { Judgement: reasons for missing data reported and review authors judge that } \\
\text { they are unlikely to be related to study outcomes }\end{array}$ \\
\hline $\begin{array}{l}\text { Selective reporting (re- } \\
\text { porting bias) }\end{array}$ & Low risk & Study protocol available and outcomes are reported in pre-specified way \\
\hline Other bias & Low risk & The study appears to be free of other sources of bias \\
\hline
\end{tabular}

Chanruengvanich 2006

\section{Study characteristics}

\begin{tabular}{|c|c|}
\hline Methods & Unit of randomisation: participant \\
\hline \multirow[t]{9}{*}{ Participants } & Place of recruitment: hospital (centre specialising in neurology) \\
\hline & Numbers randomised: total: 72; I: 36; C: 36 \\
\hline & $\%$ Completing final follow-up: $86 \%$ \\
\hline & Inclusion criteria: > 6 weeks since TIA or minor stroke; energy expenditure $<1000 \mathrm{Kcal} /$ week; age $>45$ \\
\hline & years; no cognitive impairment; able to exercise; $\mathrm{BP} \leq 180 / 100 \mathrm{mmHg}$; fasting blood sugar $\leq 150 \mathrm{mg} \%$ \\
\hline & Exclusion criteria: complications e.g. heart attack or chest pain \\
\hline & Type of stroke (\%): not reported \\
\hline & Mean age (SD): I: 62.8 (7.4); C: $63.1(7.1)$ \\
\hline & Gender (\% women): I: 68\%; C: 68\% \\
\hline
\end{tabular}


Chanruengvanich 2006 (Continued)

Ethnicity: not reported

Socio-economic or socio-demographic status:

- Marital status: single - $11 \%$, couple $-63 \%$, separated $-26 \%$

- Educational level: elementary - 53\%, high school - 21\%, vocational/college - 15\%, bachelor degree - $10 \%$, master degree $-1.6 \%$

- Income (Baht): $<5000-63 \%, 5001$ to $10,000-16 \%, 10,001$ to $15,000-8 \%, 15,001$ to $20,000-8 \%$, > $20,000-5 \%$

Interventions

Intervention details (components, length, frequency): 12 week self-regulated exercise program; first week - educational meeting (topics included disease management, diet, exercise and stress management); second week - instruction in self-regulation techniques and recommended exercises (using group demonstration and video); third week - home visit from researcher to identify problems; second to twelfth weeks - moderate exercise for a minimum of 15 minutes 2 to 3 times per day (recorded in exercise diary) with energy expenditure target 1000 kcal per week; researcher made weekly telephone calls to encourage participants to adhere to the exercise program

Location: community

Mode of delivery: patient education, home visit and telephone follow-up

Personnel responsible for delivery: researcher/investigator

Timing post-stroke: $>6$ weeks

Control: usual care

\begin{tabular}{ll}
\hline Outcomes & 12 weeks: SBP; DBP; total cholesterol; HDL \\
\hline General Information & Funding: this research was supported by the Thai Health Promotion Foundation \\
& Country of origin: Thailand \\
& Publication language: English \\
\hline Notes & Analysis method: not stated (per protocol) \\
& Risk of bias: unclear
\end{tabular}

\section{Risk of bias}

\begin{tabular}{|c|c|c|}
\hline Bias & Authors' judgement & Support for judgement \\
\hline $\begin{array}{l}\text { Random sequence genera- } \\
\text { tion (selection bias) }\end{array}$ & Unclear risk & "Each patient was randomly assigned" - method not reported \\
\hline $\begin{array}{l}\text { Allocation concealment } \\
\text { (selection bias) }\end{array}$ & Unclear risk & Not reported \\
\hline $\begin{array}{l}\text { Incomplete outcome data } \\
\text { (attrition bias) } \\
\text { All outcomes }\end{array}$ & Low risk & $\begin{array}{l}\text { Missing data reported by group } \\
\text { Attrition: I: 5/36 ( } 1 \text { withdrew; } 4 \text { illness prohibited exercise); C: } 3 / 36 \text { ( } 3 \text { withdrew) } \\
\text { Excluded from analysis: I: 0; C: } 2 / 36 \text { ( } 2 \text { excluded to balance the groups) } \\
\text { Judgement: reasons for missing data reported and review authors judge that } \\
\text { they are unlikely to be related to study outcomes }\end{array}$ \\
\hline $\begin{array}{l}\text { Selective reporting (re- } \\
\text { porting bias) }\end{array}$ & Low risk & $\begin{array}{l}\text { Study protocol available and all outcomes are reported in the pre-specified } \\
\text { way }\end{array}$ \\
\hline
\end{tabular}


Chanruengvanich 2006 (Continued)

Other bias Low risk The study appears to be free of other sources of bias

Chiu 2008

\section{Study characteristics}

\begin{tabular}{ll}
\hline Methods & Unit of randomisation: participant \\
\hline Participants & Place of recruitment: tertiary referral hospital (outpatients) \\
Numbers randomised: total: 160 (I: 80; C: 80$)$ \\
\% Completing final follow-up: not reported \\
Inclusion criteria: ischaemic stroke; national health insurance (coverage: 95\%); attending outpatient \\
clinics for > 12 months \\
Exclusion criteria: currently enrolled in other trials; terminal illness \\
Type of stroke: ischaemic stroke (100\%) \\
Mean age (SD): I: 65.7 (10.0); C: 64.8 (10.6) \\
Gender (\% women) I: $50 \% ; C: 50 \%$ \\
Ethnicity: not reported \\
Socio-economic or socio-demographic status: \\
- Education (\%): I: illiterate - 45\%, educated - 55\%; C: illiterate - 46\%, educated - 54\%
\end{tabular}

Interventions

Intervention details (components, length, frequency): monthly 1 hour pharmacist-led educational program conducted over 6 months; topics included drug effects, treatment goals, lifestyle modification, compliance and adverse effects; no scheduled monitoring of modifiable risk factors

Location: hospital

Mode of delivery: outpatient appointment

Personnel responsible for delivery: pharmacist

Timing post-stroke: $>12$ months

Control: usual care (attendance at outpatient clinics)

Outcomes 6 months: SBP; DBP; total cholesterol; LDL; triglycerides; BP < 140/90 mmHg; LDL <100 mg/dL or TC < $160 \mathrm{mg} / \mathrm{dL} ; \mathrm{HbA} 1 \mathrm{c}<7 \%$ or fasting blood glucose $<126 \mathrm{mg} / \mathrm{dL}$ or random postprandial blood glucose $<$ $200 \mathrm{mg} / \mathrm{dL}$

\begin{tabular}{ll}
\hline General Information & Funding: not reported \\
& Country of origin: Taiwan \\
& Publication language: English
\end{tabular}

Notes Analysis method: not stated

Risk of bias: unclear

\section{Risk of bias}

Interventions for improving modifiable risk factor control in the secondary prevention of stroke (Review) 
Chiu 2008 (Continued)

Bias Authors' judgement Support for judgement

Random sequence genera- Unclear risk "Simple random sampling"
tion (selection bias)

\begin{tabular}{ll}
\hline $\begin{array}{l}\text { Allocation concealment } \\
\text { (selection bias) }\end{array}$ & Unclear risk $\quad$ Not stated \\
\hline
\end{tabular}

\begin{tabular}{lll}
\hline $\begin{array}{l}\text { Incomplete outcome data } \\
\text { (attrition bias) } \\
\text { All outcomes }\end{array}$ & Unclear risk & Missing data not reported \\
\hline $\begin{array}{l}\text { Selective reporting (re- } \\
\text { porting bias) }\end{array}$ & Unclear risk & Insufficient information (protocol not obtained) \\
\hline Other bias & Low risk & The study appears to be free of other sources of bias \\
\hline
\end{tabular}

Damush 2015

\section{Study characteristics}

\begin{tabular}{ll}
\hline Methods & RCT \\
& Unit of randomisation: participant
\end{tabular}

\begin{tabular}{|c|c|}
\hline Participants & 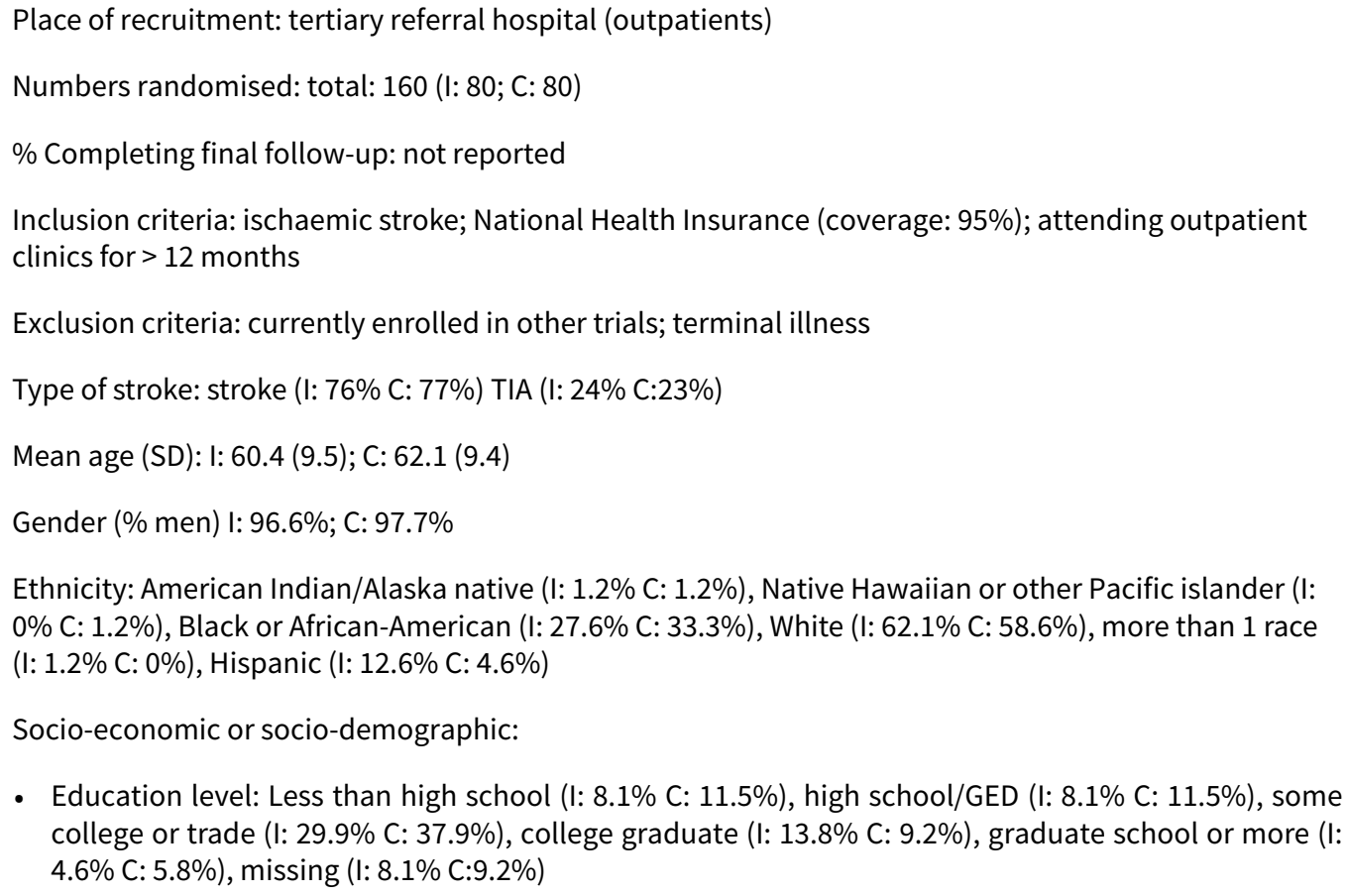 \\
\hline Interventions & $\begin{array}{l}\text { Intervention details (components, length, frequency): Up to } 6 \text { bi-weekly telephone services to deliver a } \\
\text { stroke self management program, based on Stanford chronic disease self-management program } \\
\text { Location: outpatient } \\
\text { Mode of delivery: telephone } \\
\text { Personnel responsible for delivery: nurse case manager }\end{array}$ \\
\hline
\end{tabular}


Damush 2015 (Continued)

Timing post-stroke: 6 months

Control: usual care

\begin{tabular}{|c|c|}
\hline Outcomes & 6 months: medication adherence \\
\hline \multirow[t]{3}{*}{ General Information } & $\begin{array}{l}\text { Funding: this study was funded by the VA HSRD Investigator Initiated Research Grant IAB 05-297-2 and } \\
\text { by the HSRD VA Stroke QUERI Center }\end{array}$ \\
\hline & Country of origin: USA \\
\hline & Publication language: English \\
\hline \multirow[t]{2}{*}{ Notes } & Analysis method: repeated measured logistic regression \\
\hline & Risk of bias: unclear \\
\hline
\end{tabular}

\section{Risk of bias}

\begin{tabular}{lll}
\hline Bias & Authors' judgement & Support for judgement \\
\hline $\begin{array}{l}\text { Random sequence genera- } \\
\text { tion (selection bias) }\end{array}$ & Low risk & Computer-generated assignment, stratified by stroke versus TIAs \\
\hline $\begin{array}{l}\text { Allocation concealment } \\
\text { (selection bias) }\end{array}$ & Low risk & Central allocation \\
\hline $\begin{array}{l}\text { Incomplete outcome data } \\
\text { (attrition bias) } \\
\text { All outcomes }\end{array}$ & Unclear risk & The study did not address this outcome \\
\hline $\begin{array}{l}\text { Selective reporting (re- } \\
\text { porting bias) }\end{array}$ & Unclear risk & Insufficient information to permit judgement \\
\hline \begin{tabular}{l} 
Other bias \\
\hline
\end{tabular} & Low risk & The study appears to be free of other sources of bias \\
\hline
\end{tabular}

Dregan 2014

\section{Study characteristics}

\begin{tabular}{ll}
\hline Methods & RCT \\
& Unit of randomisation: family practices \\
\hline Participants & Place of recruitment: primary care \\
& Numbers randomised: total: 11,391 (I: 5875; C: 5516) \\
& Completing final follow-up: $90 \%$ \\
& Inclusion criteria: $\geq 18$ years, included on the practice stroke register \\
& Exclusion criteria: none stated \\
& Type of stroke (\%): haemorrhagic (I: 18 C: 16$)$, ischaemic (I: 26 C: 21), undefined (I: 56 C: 63$)$ \\
& Mean age (SD): I: 72.9 (14.1); C: 72.2 (13.9) \\
& Gender (\% women) I: 49 C: 47
\end{tabular}


Dregan 2014 (Continued)

Ethnicity: not stated

Socio-economic or socio-demographic: not stated

Intervention details (components, length, frequency): educational and decision support tools for primary care healthcare providers, taken from evidence summarised from guidelines, including clinical trials, meta-analysis and observational analysis - prompts for BP/cholesterol level/statins/anticoagulant assessment.

Mode of delivery: delivered remotely via point of care software for use in the community

Personnel responsible for delivery: software system

Timing post-stroke: unlimited

Control: usual care

\begin{tabular}{|c|c|}
\hline Outcomes & 12 months: BP and total cholesterol levels \\
\hline \multirow[t]{3}{*}{ General Information } & $\begin{array}{l}\text { Funding: the study was supported by the Joint Initiative in Electronic Patient Records and Databases in } \\
\text { Research, a partnership between the Wellcome Trust, Medical Research Council, Economics and Social } \\
\text { Research Council, and Engineering and Physical Sciences Research Council }\end{array}$ \\
\hline & Country of origin: UK \\
\hline & Publication language: English \\
\hline
\end{tabular}

Notes

Analysis method: marginal methods estimated using the method of generalised estimating equations

Risk of bias: Low risk

\section{Risk of bias}

\begin{tabular}{lll}
\hline Bias & Authors' judgement & Support for judgement \\
\hline $\begin{array}{ll}\text { Random sequence genera- } \\
\text { tion (selection bias) }\end{array}$ & Low risk & "The allocation is by minimization controlling for region in England (North \\
& & (North-East and North-West), Midlands (East and West Midlands), South-East \\
& (South-East and East of England), South-West, and London) and country in the \\
& UK (Scotland, Wales, England) and list size (number of registered patients). \\
& This list size was dichotomized for the minimization using 7,500 as the cut- \\
& point. The allocation is performed at King's College London using anonymised \\
& practice identifiers supplied by the recruitment team at GPRD/MHRA"
\end{tabular}

Allocation concealment Low risk Allocation was performed using anonymised practice identifiers

(selection bias)

Incomplete outcome data Low risk

(attrition bias)

Risk of bias is acceptable - no added value is obvious from the results. Sensitivity analysis was also undertaken

All outcomes

$\begin{aligned} & \text { Selective reporting (re- } \quad \text { Low risk } \\ & \text { porting bias) }\end{aligned}$

\begin{tabular}{ll}
\hline Other bias $\quad$ Low risk $\quad$ The study appeared to be free of other sources of bias \\
\hline
\end{tabular}

\section{Study characteristics}

Interventions for improving modifiable risk factor control in the secondary prevention of stroke (Review) 
Eames 2013 (Continued)

Methods RCT

Unit of randomisation: participant

Participants

Place of recruitment: 2 acute stroke units in metropolitan hospitals

Numbers randomised: total: 77 (I:37; C: 40)

$\%$ Completing final follow-up: $86 \%$

Inclusion criteria: ischaemic stroke, haemorrhagic stroke or TIA; admitted to hospital for stroke or TIA; living in a residential care facility prior to admission and it was not a planned discharge destination; adequate spoken English, cognition, communication and corrected vision and hearing to complete the outcome measures

Exclusion criteria: poor medical prognosis (i.e. medically unstable patients or those undergoing palliative treatment)

Type of stroke: ischaemic (I: 73\%; C: 84\%); haemorrhagic (I: 25\%; C: 14\%), TIA (I:3\%, C: 0\%)

Mean age (SD): I: 57.0 (16.6); C: 64.1 (14.3)

Gender (\% men) I: 55\%; C: 51\%

Ethnicity: not reported

Socio-economic or socio-demographic status: not reported

Interventions

Intervention details (components, length, frequency): tailored written stroke information (stroke booklet) and verbal reinforcement of this information by a health professional (verbal reinforcement was offered face-to-face up to 3 times prior to discharge and over the telephone up to 3 times following discharge). Participants could tailor the content of the information booklet and the verbal sessions

Location: acute stroke unit (prior to discharge) and community/inpatient rehabilitation ward (post-discharge)

Mode of delivery: outpatient appointment

Personnel responsible for delivery: occupational therapist

Timing post-stroke: approximately 1 week prior to acute stroke unit discharge

Control: usual care (stroke unit care included usual medical, nursing, and allied health management)

Outcomes 3 months: adherence to secondary prevention medications

\begin{tabular}{ll}
\hline General Information & Funding: none received \\
& Country of origin: Australia \\
& Publication language: English \\
\hline Notes & Analysis method: unknown \\
& Risk of bias: low \\
\hline
\end{tabular}

\section{Risk of bias}

Bias Authors' judgement Support for judgement


Eames 2013 (Continued)

Random sequence genera- Low risk "Concealed, random allocation was achieved via sequentially numbered en tion (selection bias) velopes containing computer-generated random numbers prepared by a person not involved in the study"

$\begin{array}{ll}\begin{array}{l}\text { Allocation concealment } \\ \text { (selection bias) }\end{array} & \text { "Baselines outcome measures were obtained prior to randomization and } \\ & \text { therefore by a blinded assessor. Administration of outcome measures at the } \\ \text { follow-up interview was undertaken by a blinded assessor. Once completed, } & \text { the assessor opened a sealed section of the form to determine group alloca- } \\ \text { tion and asked intervention group participants additional questions regarding } & \text { the intervention." (Unpublished information provided by trialists) }\end{array}$

Incomplete outcome data Low risk
(attrition bias)

Missing data reported by group

All outcomes

Attrition: I: 5/40 (4 unable to be contacted; 1 cognition impairment too severe for interview follow-up; C:6/37 ( 2 withdrew; 3 unable to be contacted; 1 admitted to residential care

Judgement: reasons for missing data reported and review authors judge that they are unlikely to be related to study outcomes

Selective reporting (re- Low risk Protocol is available and outcomes are reported in the pre-specified way
porting bias)

\begin{tabular}{ll}
\hline Other bias Low risk The study appears to be free of other sources of bias \\
\hline
\end{tabular}

Ellis 2005

\section{Study characteristics}

Methods RCT

Unit of randomisation: participant

\begin{tabular}{|c|c|}
\hline \multirow[t]{10}{*}{ Participants } & Place of recruitment: hospital TIA clinic or geriatric medical day hospital \\
\hline & Numbers randomised: total: 205 (I: 100; C: 105) \\
\hline & $\%$ Completing final follow-up: $94 \%$ \\
\hline & $\begin{array}{l}\text { Inclusion criteria: }<3 \text { months since stroke, TIA or amaurosis fugax; ambulant patients; one of more car- } \\
\text { diovascular risk factor (high BP, history of current smoking, high cholesterol, diabetes) }\end{array}$ \\
\hline & Exclusion criteria: cognitive impairment (AMT < 5 on screening) \\
\hline & Type of stroke: TIA (I: 29\%; C: 26\%); stroke (I: 61\%; C: 65\%) \\
\hline & Mean age ( $95 \% \mathrm{Cl})$ : I: 64.3 (62.4 to 66.1$)$, C: 65.8 (64.0 to 67.5$)$ \\
\hline & Gender (\% men): I: 54\%; C: 50\% \\
\hline & Ethnicity: not reported \\
\hline & Socio-economic or socio-demographic status: not reported \\
\hline \multirow[t]{2}{*}{ Interventions } & $\begin{array}{l}\text { Intervention details (components, length, frequency): monthly reviews (approximately } 3 \text { ) with a stroke } \\
\text { nurse specialist; participants received tailored verbal and written information addressing medication } \\
\text { compliance, lifestyle modification, interaction with medical services, risk factor status and risk factor } \\
\text { targets; participants advised to visit their GP if risk factors poorly controlled }\end{array}$ \\
\hline & Location: hospital outpatient setting \\
\hline
\end{tabular}


Ellis 2005 (Continued)

Mode of delivery: outpatient appointment

Personnel responsible for delivery: stroke nurse specialist

Timing post-stroke: first review at 3 months

Control: usual care (1 review in hospital outpatient setting where patients received standard outpatient advise on risk factors and secondary prevention; discharged to general practice care)

Outcomes

5 months (per protocol): SBP; DBP; total cholesterol; HbA1c; combined risk factor control

3.6 years (additional follow-up): SBP; DBP; total cholesterol; HbA1c; persistence with therapy; self-reported adherence; recurrent cardiovascular events; percentage of patients meetings target for combined risk factor control

\begin{tabular}{ll}
\hline General Information & Funding: educational grant from Servier Laboratories \\
Country of origin: UK \\
Publication language: English
\end{tabular}

Notes Analysis method: stated intention-to-treat

Risk of bias: unclear

\section{Risk of bias}

Bias Authors' judgement Support for judgement

Random sequence genera- Low risk "Patients were randomly allocated to treatment or control groups using a tion (selection bias) computer-generated random sequence"

Allocation concealment Low risk "Concealed in sequentially numbered opaque sealed envelopes"
(selection bias)

\begin{tabular}{ll}
$\begin{array}{l}\text { Incomplete outcome data } \\
\text { (attrition bias) }\end{array}$ & Missing data reported by group \\
All outcomes & $\begin{array}{l}\text { Attrition: I: } 6 \text { lost to follow-up (reasons unclear); C: } 7 \text { lost to follow-up (reasons } \\
\text { unclear) }\end{array}$ \\
& $\begin{array}{l}\text { Excluded from analysis: I: } 3 \text { patients entered twice by error: duplicate results } \\
\text { excluded from the analysis; } 1 \text { patient found to be ineligible: results included } \\
\text { in the analysis (intention-to-treat) }\end{array}$ \\
& $\begin{array}{l}\text { Judgement: reasons for missing data reported and review authors judge that } \\
\text { they are unlikely to be related to study outcomes }\end{array}$ \\
\hline
\end{tabular}

\begin{tabular}{l}
$\begin{array}{l}\text { Selective reporting (re- Unclear risk } \\
\text { porting bias) }\end{array}$ \\
\hline
\end{tabular}

Other bias Low risk The study appears to be free of other sources of bias

Evans 2010

\section{Study characteristics}

$\begin{array}{ll}\text { Methods } & \text { RCT } \\ & \text { Unit of randomisation: participant }\end{array}$


Evans 2010 (Continued)

Participants
Place of recruitment: primary care medical clinic

Numbers randomised: total: 176 (8 stroke/TIA); I: 88 (4 stroke/TIA); C: 88 (4 stroke/TIA)

\% Completing final follow-up: $89 \%$

Inclusion criteria: Framingham risk score $\geq 15 \%$ or coronary artery disease risk equivalent (coronary artery disease, peripheral artery disease, cerebrovascular disease, diabetes mellitus)

Exclusion criteria: severe psychiatric conditions or demential symptomatic heart failure; terminal illness

Type of stroke (\%): not stated

Mean age (SD): 62.5 (10.5)

Gender (\% men): $87.5 \%$

Ethnicity: not reported

Socio-economic or socio-demographic status: not reported
Intervention details (components, length, frequency): pharmacist-delivered secondary prevention program involving cardiovascular risk stratification, monitoring of cardiovascular risk factors and drug adherence support; participants were contacted approximately every 8 weeks for minimum of 6 months (telephone call, appointment, mailed letters); mean duration of follow-up was 380 days; participants and their primary care physicians were informed if risk factors were uncontrolled

Location: primary care medical clinic

Mode of delivery: primary care appointment

Personnel responsible for delivery: pharmacist (intervention designed for non-specialist pharmacists to facilitate collaborative partnerships without the need for advanced training)

Timing post-stroke: unknown

Usual care (I and C): general counselling about cardiovascular disease (1 hour pharmacist appointment)

\section{Outcomes}

12 months: SBP; DBP; total cholesterol; LDL; HDL; triglycerides; HbA1C; 10 year Framingham risk score
Funding: funding through a Canadian Institute of Health Research (CIHR) Clinical Research Initiative Fellowship and funding for salary support award from the Alberta Heritage Foundation for Medical Research

Country of origin: Canada

Publication language: English

\section{Notes}

Analysis method: stated intention-to-treat

Risk of bias: low

\section{Risk of bias}

\section{Bias}

Authors' judgement Support for judgement

Random sequence genera- Low risk tion (selection bias)

"Randomisation lists were stratified by each physician and were created by using a table of random numbers in permuted blocks of four" 
Evans 2010 (Continued)

\begin{tabular}{|c|c|c|}
\hline $\begin{array}{l}\text { Allocation concealment } \\
\text { (selection bias) }\end{array}$ & Low risk & $\begin{array}{l}\text { "Randomisation codes were kept in individually sealed envelopes and opened } \\
\text { by the study pharmacist at the end of the initial visit" }\end{array}$ \\
\hline
\end{tabular}

Incomplete outcome data Low risk

(attrition bias)

Missing data reported by group

All outcomes

Attrition: I: 11/88 (9 laboratory data not available; 1 moved; 1 died); C: 9/88 (8 laboratory data not available; 1 withdrew due to unrelated illness)

Judgement: reasons for missing data reported and review authors judge that they are unlikely to be related to study outcomes

Selective reporting (re- Low risk Protocol available and outcomes reported in the pre-specified way
porting bias)

Other bias Low risk The study appears to be free of other sources of bias

Flemming 2013

\section{Study characteristics}

Methods RCT

Unit of randomisation: participant

Place of recruitment: hospital
Numbers randomised: total: 41 (I: 20; C: 21$)$
\% Completing final follow-up: $88 \%$
Inclusion criteria: ischaemic stroke or TIA and at least one uncontrolled strol
sion, hyperlipidaemia, diabetes or tobacco use); > 55 years old
Exclusion criteria: NIHSS > 7; prior enrolment in cardiovascular prevention
year
Type of stroke (\%): TIA (I: $40 \%$, C: $52 \%)$; ischaemic stroke (I: $60 \%$, C: $48 \%)$
Mean age (SD): I: 70 (13); C: 71 (9)
Gender (\% men): I: $50 \% ; C: 66 \%$
Ethnicity: not reported
Socio-economic or socio-demographic status: not reported

Interventions Intervention details (components, length, frequency): nurses were trained in stroke risk factors and motivational interviewing; participants attended nurse-led appointments for risk factor review (baseline, 6 weeks, 6 months and 1 year) and received additional nurse-led telephone follow-up; nurses followed standardised protocols for the assessment and management of stroke risk factors; participants attended consultations with dietician and exercise physiologist; secondary stroke prevention recommendations and participants' risk factor assessments were sent to their $\mathrm{GP} /$ neurologist

Location: outpatient clinic

Mode of delivery: outpatient appointment and telephone follow-up

Personnel responsible for delivery: nurses

Timing post-stroke: $<12$ weeks 
Flemming 2013 (Continued)

Usual care (I and C): usual care: baseline risk factor assessment and follow-up appointment (1 year); usual follow-up by primary care/neurology

\begin{tabular}{|c|c|}
\hline Outcomes & $\begin{array}{l}12 \text { months: change in cardiovascular risk factors (SBP; LDL; HDL; triglycerides; HbA1c; BMI; Framing- } \\
\text { ham cardiovascular risk score); achievement of targets for cardiovascular risk factors; number of vascu- } \\
\text { lar events; adherence to secondary prevention medication }\end{array}$ \\
\hline \multirow[t]{3}{*}{ General Information } & $\begin{array}{l}\text { Funding: this research was funded by the American Heart Association (Scientist Development Grant). } \\
\text { This research was partially funded by the Center for Translational Science Activities (CTSA) at Mayo } \\
\text { Clinic }\end{array}$ \\
\hline & Country of origin: USA \\
\hline & Publication language: English \\
\hline
\end{tabular}

\begin{tabular}{ll}
\hline Notes & Analysis method: available case analysis \\
Risk of bias: low
\end{tabular}

\section{Risk of bias}

\begin{tabular}{|c|c|c|}
\hline Bias & Authors' judgement & Support for judgement \\
\hline $\begin{array}{l}\text { Random sequence genera- } \\
\text { tion (selection bias) }\end{array}$ & Low risk & Shuffling envelopes \\
\hline $\begin{array}{l}\text { Allocation concealment } \\
\text { (selection bias) }\end{array}$ & Low risk & Envelope method \\
\hline \multirow{3}{*}{$\begin{array}{l}\text { Incomplete outcome data } \\
\text { (attrition bias) } \\
\text { All outcomes }\end{array}$} & Low risk & Missing data reported by group \\
\hline & & Attrition: I: 2/20 (1 died; 1 lost to follow-up); C:3/21 (2 died; lost to follow-up) \\
\hline & & $\begin{array}{l}\text { Judgement: reasons for missing data reported and review authors judge that } \\
\text { they are unlikely to be related to study outcomes }\end{array}$ \\
\hline $\begin{array}{l}\text { Selective reporting (re- } \\
\text { porting bias) }\end{array}$ & Low risk & Protocol available and outcomes reported in the pre-specified way \\
\hline Other bias & Low risk & The study appears to be free of other sources of bias \\
\hline
\end{tabular}

\section{Hanley 2015}

\section{Study characteristics}

Methods RCT

Unit of randomisation: participant

Participants Place of recruitment: GP surgery

Numbers randomised: total: 55 (I: 40; C: 15)

$\%$ Completing final follow-up: $95 \%$

Inclusion criteria: all stroke and TIA, > 18 years, systolic BP $>130 \mathrm{mmHg}$

Exclusion criteria: secondary hypertension, hypertension managed by secondary care, surgery BP < $120 / 60$ or $>220$ systolic at baseline, major surgery in last 3 months, unable to give consent, unable to 
Hanley 2015 (Continued)

use home blood pressure monitor, terminal illness, major concurrent illness, AF, stroke within the last 3 months

Type of stroke (\%): TIA (I: 50\%, C: 47\%); ischaemic stroke (I: 50\%, C: 53\%)

Mean age (SD): I: 69.9 (12.6); C: 73.5 (11.7)

Gender (\% men): I: $68 \%$; C: $40 \%$

Ethnicity: not reported

Socio-economic or socio-demographic status: not reported

Interventions

Intervention details (components, length, frequency): participants measured their own BP, including reminders to self monitor, sent readings to GP via Bluetooth, checked by practice nurse, with telephone or face-to-face appointments made as needed. Participants were given information on lifestyle measures to reduce $\mathrm{BP}$

Location: community

Mode of delivery: remote

Personnel responsible for delivery: nurse

Timing post-stroke: $>3$ months after a stroke/TIA

Control: usual care

\begin{tabular}{ll}
\hline Outcomes & 6 months: ambulatory BP \\
\hline General Information & Funding: this study was funded by the Chief Scientist Office (CSO), Scottish Government \\
& Country of origin: UK \\
& Publication language: English \\
\hline
\end{tabular}

Notes

Analysis method: as this was a feasibility study, no statistical analysis was undertaken

Risk of bias: unclear

\section{Risk of bias}

\begin{tabular}{lll}
\hline Bias & Authors' judgement & Support for judgement \\
\hline $\begin{array}{l}\text { Random sequence genera- } \\
\text { tion (selection bias) }\end{array}$ & Low risk & $\begin{array}{l}\text { Randomisation undertaken using a 3:1 ratio using a remote Internet-based } \\
\text { system provided by the Edinburgh Clinical Trials Unit }\end{array}$ \\
\hline $\begin{array}{l}\text { Allocation concealment } \\
\text { (selection bias) }\end{array}$ & Low risk & Central allocation \\
\hline $\begin{array}{l}\text { Incomplete outcome data } \\
\text { (attrition bias) } \\
\text { All outcomes }\end{array}$ & Unclear risk & The study did not address this risk of bias \\
\hline $\begin{array}{l}\text { Selective reporting (re- } \\
\text { porting bias) }\end{array}$ & Unclear risk & $\begin{array}{l}\text { As this was a pilot, the methods are described but not published elsewhere } \\
\text { with pre-specified outcomes }\end{array}$ \\
\hline \begin{tabular}{l} 
Other bias \\
\hline
\end{tabular} & Low risk & The study appears to be free of other sources of bias \\
\hline
\end{tabular}


Hedegaard 2014

\section{Study characteristics}

$\begin{array}{ll}\text { Methods } & \text { RCT } \\ \text { Unit of randomisation: participant }\end{array}$

Participants

Place of recruitment: emergency ward or from 5 locations - 2 inpatient wards, 1 patient hotel, 1 rehabilitation centre and 1 TIA outpatient clinic

Numbers randomised: total: 211 (I: 104; C: 107)

$\%$ Completing final follow-up: $96 \%$

Inclusion criteria: ischaemic stroke or TIA within the previous 30 days, acute first stroke, $>18$ years of age, prescribed at least 1 antiplatelet or anticoagulant medication, participant or co-habiting relatives dispensed the participant's medications

Exclusion criteria: lives in a care home or institution, dose dispersed medications from a pharmacy, if medication was dispensed by a home nurse, terminal illness or cognitive/physical impairment

Type of stroke: TIA (I: 47\%; C: 49\%); ischaemic stroke (I: 52\%; C: 50\%)

Mean age (range): I: 64 (56-73), C: 68 (61-73)

Gender (\% men): I: 59.8; C: 62.4

Ethnicity: not reported

Socio-economic or socio-demographic status: not reported

Intervention details (components, length, frequency): clinical pharmacists were trained in providing 1) a focused medication review followed by dialogue based on motivational interviewing to support adherence and lifestyle changes; 2 ) a patient interview followed by a list of their own goals and agreed actions; 3 ) 3 follow-up telephone calls to the participant ( 1 week, 2 months and 6 months) where participants were given a written summary of their goals and plans after the second and third calls

Location: outpatient clinic

Mode of delivery: outpatient appointment and telephone follow-up

Personnel responsible for delivery: pharmacists

Timing post-stroke: within 30 days

Usual care (I and C): usual care without the clinical pharmacist. 2 months after the start of the study, a secondary prevention clinic was initiated for all participants with follow-up from a stroke specialist nurse, including baseline risk factor assessment, medication adherence and lifestyle behaviour at day 14 and 3 months

Outcomes

Overall adherence to thrombo-preventative regimen in the year after hospitalisation based on the medication adherence ratio

\section{General Information}

Funding: the work was funded by grants from Odense University Hospital, the University of Southern Denmark, the hospital pharmacies and the Amgros I/S Reserach development foundation as well as Actavais Foundation

Country of origin: Denmark

Publication language: English

Notes

Analysis method: exploratory per-protocol analysis

Risk of bias: unclear 
Hedegaard 2014 (Continued)

Risk of bias

\begin{tabular}{|c|c|c|}
\hline Bias & Authors' judgement & Support for judgement \\
\hline $\begin{array}{l}\text { Random sequence genera- } \\
\text { tion (selection bias) }\end{array}$ & Low risk & $\begin{array}{l}\text { Performed by clinical trial group at hospital pharmacy. } 1: 1 \text { allocation, ran- } \\
\text { domised in blocks of } 4 \text { and } 6 \text { by computer prior to enrolment and concealed in } \\
\text { opaque envelopes }\end{array}$ \\
\hline $\begin{array}{l}\text { Allocation concealment } \\
\text { (selection bias) }\end{array}$ & Low risk & Central allocation with opaque envelopes \\
\hline $\begin{array}{l}\text { Incomplete outcome data } \\
\text { (attrition bias) } \\
\text { All outcomes }\end{array}$ & Unclear risk & The study did not address this outcome \\
\hline $\begin{array}{l}\text { Selective reporting (re- } \\
\text { porting bias) }\end{array}$ & Unclear risk & Insufficient information to permit judgement. Protocol available \\
\hline Other bias & Low risk & The study appears to be free of other sources of bias \\
\hline
\end{tabular}

Hornnes 2011

\section{Study characteristics}

\begin{tabular}{|c|c|}
\hline Methods & $\begin{array}{l}\text { RCT } \\
\text { Unit of randomisation: participant }\end{array}$ \\
\hline Participants & 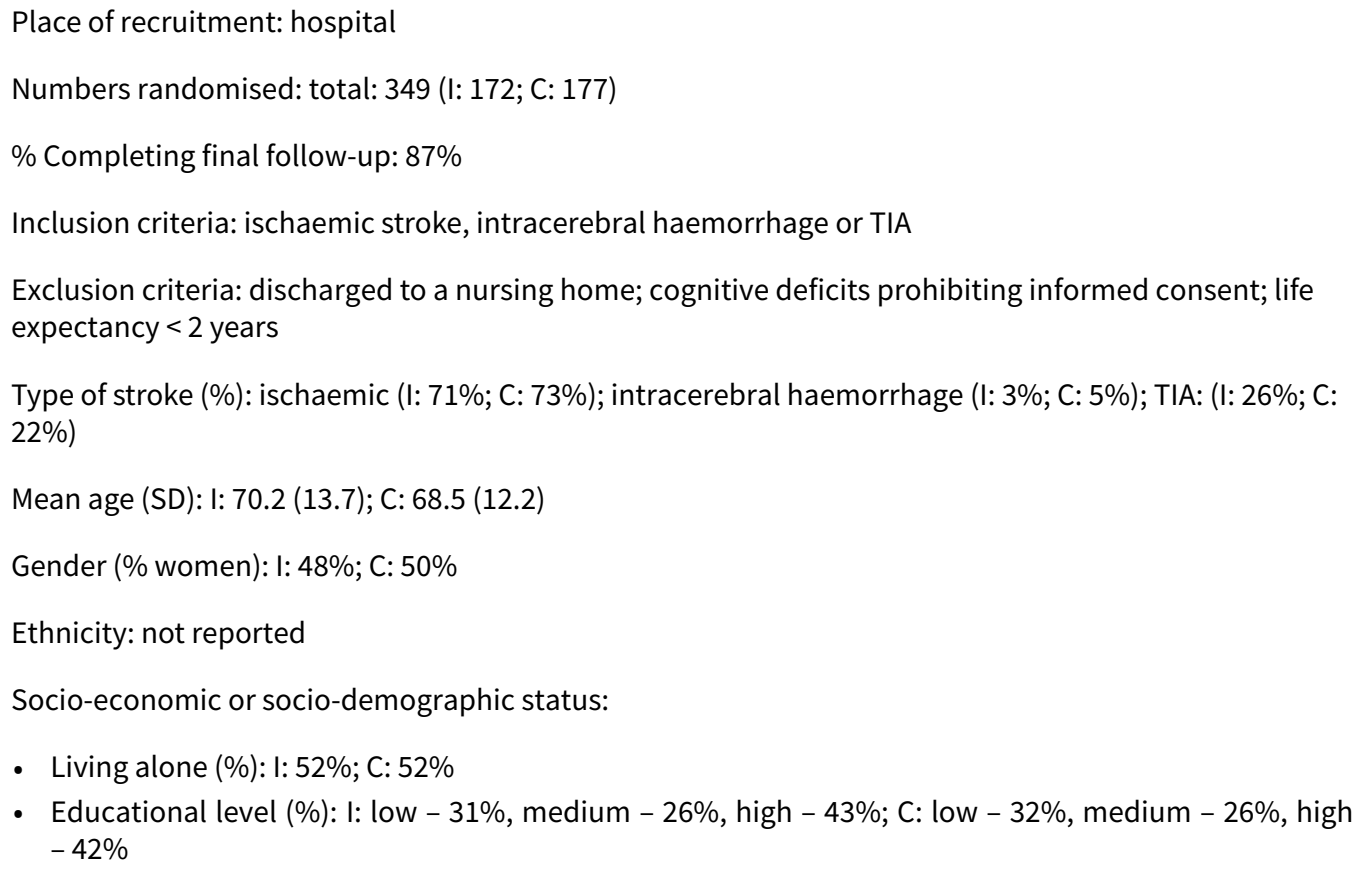 \\
\hline Interventions & $\begin{array}{l}\text { Intervention details (components, length, frequency): } 4 \text { home visits from a nurse at } 1,4,7 \text { and } 10 \\
\text { months; each visit included blood pressure monitoring, tailored lifestyle counselling and promotion of } \\
\text { medication compliance; hypertensive participants encouraged to visit their GP } \\
\text { Location: community }\end{array}$ \\
\hline
\end{tabular}


Hornnes 2011 (Continued)

Mode of delivery: home visits

Personnel responsible for delivery: nurse

Timing post-stroke: randomised at time of discharge

Control: usual care (neurologist outpatient visit 3 months post-stroke)

Outcomes 12 months: SBP; DBP; proportion of participants meeting BP targets; proportion of participants adher-
ing antihypertensive therapy

General Information $\quad$ Funding: funding support from Servier Danmark A/S and the Lundbeck Foundation
Country of origin: Denmark
Publication language: English

Notes Analysis method: not reported

Risk of bias: low

\begin{tabular}{|c|c|c|}
\hline \multicolumn{3}{|l|}{ Risk of bias } \\
\hline Bias & Authors' judgement & Support for judgement \\
\hline $\begin{array}{l}\text { Random sequence genera- } \\
\text { tion (selection bias) }\end{array}$ & Low risk & "Used a computer-generated, block randomization procedure" \\
\hline $\begin{array}{l}\text { Allocation concealment } \\
\text { (selection bias) }\end{array}$ & Low risk & $\begin{array}{l}\text { "The allocation sequence was concealed ... the study nurses who adminis- } \\
\text { tered the intervention had access to a computer program ... entering the pa- } \\
\text { tient's Central Person Registry number, BP value, and hospital yielded a print- } \\
\text { out of the patient's randomization number and allocation" }\end{array}$ \\
\hline \multirow{3}{*}{$\begin{array}{l}\text { Incomplete outcome data } \\
\text { (attrition bias) } \\
\text { All outcomes }\end{array}$} & Low risk & Missing data reported by group \\
\hline & & $\begin{array}{l}\text { Attrition: I: } 27 / 172 \text { ( } 13 \text { dropped out; } 3 \text { diagnosis revised; } 10 \text { died; } 1 \text { too ill); C: } \\
\text { 19/177 (9 dropped out; } 5 \text { died; } 2 \text { too ill; } 2 \text { diagnosis revised; } 1 \text { other reason) }\end{array}$ \\
\hline & & $\begin{array}{l}\text { Judgement: reasons for missing data reported and review authors judge that } \\
\text { they are unlikely to be related to study outcomes }\end{array}$ \\
\hline $\begin{array}{l}\text { Selective reporting (re- } \\
\text { porting bias) }\end{array}$ & Low risk & $\begin{array}{l}\text { Outcomes pre-specified (trial registry: www.clinicaltrials.gov/ct2/show/ } \\
\text { NCT00253097) }\end{array}$ \\
\hline Other bias & Low risk & The study appears to be free of other sources of bias \\
\hline
\end{tabular}

Johnston 2010

\section{Study characteristics}

\begin{tabular}{ll}
\hline Methods & RCT \\
& Unit of randomisation: hospital \\
\hline
\end{tabular}

Place of recruitment: 12 hospitals
Numbers randomised: total: 3361 (I: 1464; C: 1897$)$
\% Completing final follow-up: $80 \%$


Inclusion criteria: ischaemic stroke; Kaiser Permanente Medical Care Plan members with pharmacy benefits; age $\geq 40$ years; acute hospitalisation for stroke

Exclusion criteria: haemorrhagic stroke; discharged to hospice

Type of stroke: ischaemic (100\%)

Mean age (SD): 72.9 (12.6)

Gender (\% women): 53\%

Ethnicity: non-Hispanic white 66\%; African American 14\%; Asian/Pacific Islander 11\%; Hispanic 7\%; other/unknown $1 \%$

Socio-economic or socio-demographic status: members of Kaiser Permanente Medical Care Plan with "under-representation of the very poor and wealthy"

Interventions

Intervention details (components, length, frequency): hospitals received support from a central coordinator in the development and implementation of standardised stroke discharge orders (discharge orders based on American Heart Association recurrent stroke prevention guidelines and included 1) statin prescription for all patients irrespective of cholesterol levels; 2) antihypertensive prescriptions for hypertensive patients; 3) warfarin prescription for patients with atrial fibrillation); 2 physician 'champions' (from neurology and hospital-based medicine) from each hospital tailored discharge order and supervised implementation; 2 educational presentations delivered to healthcare providers (timing: development of discharge orders and 3 months post-implementation)

Location: Kaiser Permanente Medical Care Plan hospitals

Mode of delivery: health provider education and pre-printed stroke discharge orders

Personnel responsible for delivery: central co-ordinator and 2 physicians supervised implementation

Timing post-stroke: discharge from hospital

Control: usual care without contact from study staff; some hospitals implemented their own discharge orders

$\begin{array}{ll}\text { Outcomes } & \begin{array}{l}6 \text { months: } \mathrm{BP}<140 / 90 \mathrm{mmHg} \text {; combined cardiovascular risk factor control; adherence to secondary } \\ \text { prevention medications }\end{array}\end{array}$

General Information Funding: Centres for Disease Control and Prevention, administered through the Association of American Medical Colleges

Country of origin: USA

Publication language: English

\begin{tabular}{ll}
\hline Notes & Analysis method: stated intention-to-treat \\
& Risk of bias: unclear
\end{tabular}

\section{Risk of bias}

\begin{tabular}{lll}
\hline Bias & Authors' judgement & Support for judgement \\
\hline $\begin{array}{l}\text { Random sequence genera- } \\
\text { tion (selection bias) }\end{array}$ & Low risk & $\begin{array}{l}\text { "Participating hospitals were paired based on characteristics that could have } \\
\text { impacted the success of the intervention, including patient demographics, } \\
\text { hospital size, number of enrollees, and presence of a motivated stroke expert. } \\
\text { Then, using a random number generator, } 1 \text { hospital in each pair was random- } \\
\text { ized to receive the intervention, whereas the other was randomized to usual } \\
\text { care." }\end{array}$ \\
\end{tabular}


Johnston 2010 (Continued)

Allocation concealment Low risk "Participating hospitals were paired based on characteristics that could have (selection bias) impacted the success of the intervention, including patient demographics, hospital size, number of enrollees, and presence of a motivated stroke expert. Then, using a random number generator, 1 hospital in each pair was randomized to receive the intervention, whereas the other was randomized to usual care."

$\begin{array}{ll}\begin{array}{l}\text { Incomplete outcome data } \\ \text { (attrition bias) }\end{array} & \text { Low risk } \\ \text { All outcomes } & \text { Attrition: I: 1149/1464 (237 died; } 78 \text { lost to follow-up); C: } 1533 / 1897 \text { (277 died; } \\ & 87 \text { lost to follow-up) } \\ & \text { Judgement: reasons for missing data reported and review authors judge that } \\ & \text { they are unlikely to be related to study outcomes }\end{array}$

\begin{tabular}{lll}
\hline $\begin{array}{l}\text { Selective reporting (re- } \\
\text { porting bias) }\end{array}$ & Unclear risk & $\begin{array}{l}\text { Protocol available and primary outcomes are reported in the pre-specified } \\
\text { way; some secondary outcomes not reported }\end{array}$ \\
\hline Other bias & Low risk & The study appears to be free of other sources of bias \\
\hline
\end{tabular}

\section{Jönsson 2014}

\section{Study characteristics}

Methods RCT

Unit of randomisation: participant

Participants

Place of recruitment: a hospital and a stroke unit

Numbers randomised: total: 459 (I: 232; C: 227)

$\%$ Completing final follow-up: $85 \%$

Inclusion criteria: first ever stroke or recurrent stroke admitted in the study period (1 February 2008 - 31 January 2009)

Exclusion criteria: not stated

Type of stroke (\%): cerebral infarct (I: 88 C: 89), intracerebral haemorrhage (I: 12 C: 11$)$

Mean age: I: 73.4 C: 73.2

Gender (\% women): I: 51 C: 51

Ethnicity: not stated

Socio-economic or socio-demographic status:

- Working - full time (I: 5.5\% C: $7 \%$ ), part-time (I: 4\% C: $2.5 \%)$, sick leave > 6 months (I: $7 \%$ C: $7 \%$ ), early retirement (I: $5.5 \%$ C: $4 \%$ ), retired (I: $77 \%$ C: $78 \%$ ), unemployed (I: $1 \%$ C: $1 \%$ ), student (I: $0 \%$ C: $0.5 \%)$

Interventions

Intervention details (components, length, frequency): participants were invited to an outpatient clinic twice to have BP/LDL undertaken at 3 months and at 1 year. The nurse offered supportive counselling regarding stroke disease, treatment, medication adherence and lifestyle advice in addition to time given for an open discussion/any questions/queries. Further interventions and referrals were made by the nurse 1) if symptoms were judged to need an acute assessment by an on-call physician including initiating treatment, 2) a non-urgent referral was needed - this was made to the GP for assessment and follow-up, 3) if the participant was a nursing home resident, further information was gained from the home nurse and appropriate referrals made to the GP 
Location: Skåne Hospital Malmö

Mode of delivery: outpatient clinic

Personnel responsible for delivery: nurse

Timing post-stroke: 3 months after the event

Control: usual care - no outlined follow-up after hospital discharge until 1 year after stroke

\begin{tabular}{|c|c|c|}
\hline Outcomes & \multicolumn{2}{|c|}{3 and 12 months values for BP, cholesterol and LDL levels, body weight, $\mathrm{HbA1}$, smoking status } \\
\hline General Information & \multicolumn{2}{|c|}{$\begin{array}{l}\text { Funding: the study was financed by the National Board of Health and Welfare to support a health devel- } \\
\text { opment program in Skane Regional Council, Sweden }\end{array}$} \\
\hline & \multicolumn{2}{|c|}{ Country of origin: Sweden } \\
\hline & \multicolumn{2}{|c|}{ Publication language: English } \\
\hline Notes & \multicolumn{2}{|c|}{ Analysis method: stated Mann-Whitney test } \\
\hline & \multicolumn{2}{|l|}{ Risk of bias: high } \\
\hline \multicolumn{3}{|l|}{ Risk of bias } \\
\hline Bias & Authors' judgement & Support for judgement \\
\hline $\begin{array}{l}\text { Random sequence genera- } \\
\text { tion (selection bias) }\end{array}$ & High risk & $\begin{array}{l}\text { Allocation was undertaken by an administration secretary using lists made by } \\
\text { a second author who used a computer generated randomised procedure with } \\
\text { stratification for age and gender }\end{array}$ \\
\hline $\begin{array}{l}\text { Allocation concealment } \\
\text { (selection bias) }\end{array}$ & Low risk & Centrally allocated computer-generated lists were used \\
\hline $\begin{array}{l}\text { Incomplete outcome data } \\
\text { (attrition bias) } \\
\text { All outcomes }\end{array}$ & Low risk & Missing data was addressed in additional information provided \\
\hline $\begin{array}{l}\text { Selective reporting (re- } \\
\text { porting bias) }\end{array}$ & Low risk & Protocol registered at Clinicaltrials.gov \\
\hline Other bias & Low risk & The study appears to be free of other sources of bias \\
\hline
\end{tabular}

Joubert 2009

\section{Study characteristics}

\begin{tabular}{ll}
\hline Methods & RCT \\
& Unit of randomisation: participant \\
\hline Participants & Place of recruitment: hospital \\
& Numbers randomised: total: 233 (I: 123; C: 110) \\
& $\%$ Completing final follow-up: $80 \%$ \\
& Inclusion criteria: ischaemic stroke, parenchymal haemorrhage or TIA; aged $\geq 20$ years
\end{tabular}


Exclusion criteria: not managed by GP; discharged to nursing home; serious co-morbidities; non-English speaking; serious cognitive impairment; significantly aphasic

Type of stroke (\%): ischaemic (I: 73\%; C: 80\%); haemorrhagic (I: 10\%: C: 7\%); TIA (I: 17\%; C: 13\%)

Mean age (SD): I: 63.4 (13.7); C: 68.2 (12.7)

Gender (\% men): I: 58\%; C: 52\%

Ethnicity: not reported

Socio-economic or socio-demographic status: not reported

Interventions

Intervention details (components, length, frequency): "shared care" program; risk factor targets derived from National guidelines and consensus statements; medication initiated in hospital; lifestyle education provided by nurse coordinator; GP appointments pre-arranged for 2 weeks, 3 months, 6 months, 9 months and 12 months post-discharge; recommendations and evidence-based guidelines sent to GP; nurse co-ordinator telephoned participants before and after every GP visit to screen for depression; risk factor data collected at each GP visit and faxed to nurse co-ordinator; nurse co-ordinator facilitated transfer of information and recommendations between stroke specialists and GPs; GPs able to telephone stroke specialist for advice

Location: community

Mode of delivery: telephone follow-up; information management

Personnel responsible for delivery: stroke specialists, a nurse co-ordinator and participants' GPs

Timing post-stroke: intervention initiated before hospital discharge

Control: standard care from GP

Outcomes 12 months: SBP; DBP, total cholesterol, BMI, systolic BP < $140 \mathrm{mmHg}$; total cholesterol < $5.18 \mathrm{mmol} / \mathrm{L}$;
proportion of $A F$ patients taking warfarin

General Information Funding: this research was funded by a Commonwealth of Australia General Practice Evaluation Program grant

Country of origin: Australia

Publication language: English

Notes Analysis method: not stated

Risk of bias: unclear

\section{Risk of bias}

\begin{tabular}{lll}
\hline Bias & Authors' judgement & Support for judgement \\
\hline $\begin{array}{l}\text { Random sequence genera- } \\
\text { tion (selection bias) }\end{array}$ & Low risk & "Computer-generated process" \\
& $\begin{array}{l}\text { "At a later stage, the coordinator checked the patient's GP, and if this GP was } \\
\text { also responsible for a different patient already in the trial, the current patient } \\
\text { was assigned to the same group as the previous patient" }\end{array}$ \\
\hline $\begin{array}{l}\text { Allocation concealment } \\
\text { (selection bias) }\end{array}$ & Low risk & $\begin{array}{l}\text { "The allocation to group was undertaken after consent, so the coordinator was } \\
\text { unaware of treatment allocation prior to consent" }\end{array}$ \\
\hline $\begin{array}{l}\text { Incomplete outcome data } \\
\text { (attrition bias) } \\
\text { All outcomes }\end{array}$ & Low risk & $\begin{array}{l}\text { Missing data reported by group } \\
\text { Attrition: I: } 32 / 123 \text { (7 unwilling to participate; } 2 \text { withdrew due to other med- } \\
\text { ical problems, } 2 \text { changed GP; } 11 \text { withdrew for unknown reasons; } 3 \text { did not have }\end{array}$ \\
\hline
\end{tabular}


stroke; 3 not contactable; 2 died; 1 moved to nursing home; 1 GP refused); C: 15/110 ( 2 unwilling to participate; 1 left country; 3 withdrew for unknown reasons; 2 did not have stroke; 1 not contactable; 6 died)

Judgement: imbalances in missing data between the groups; however the review authors judged that this was unlikely to be related to study outcomes

Selective reporting (re- Unclear risk Insufficient information (protocol not obtained)
porting bias)

Other bias Low risk The study appears to be free of other sources of bias

\section{Kerry 2013}

\section{Study characteristics}

\begin{tabular}{ll}
\hline Methods & RCT \\
& Unit of randomisation: participant \\
\hline
\end{tabular}

\section{Participants}

Place of recruitment: outpatient and inpatient stroke clinics

Numbers randomised: total: 381 (I: 187; C: 194)

$\%$ Completing final follow-up: $88 \%$

Inclusion criteria: $\leq 9$ months since stroke or TIA and hypertension (BP $>140 / 85$ mmHg or treatment with antihypertensive medications)

Exclusion criteria: enrolled in another trial; severely ill or too frail; already using a blood pressure monitor; severe cognitive impairment; non-English speaking

Type of stroke (\%): ischaemic (I: 58\%; C: 64\%); haemorrhagic (I: 7\%; C: 5\%); TIA (I: 34\%; C: 30\%); both types of stroke or unknown (I: $1 \%$; C: $2 \%)$

Mean age (SD): I: 71.1 (12.6); C: 72.6 (11.4)

Gender (\% men): I: 59\%; C: $56 \%$

Ethnicity: White (I: 80\%; C: 73\%); Black (I: 11\%; C: 15\%); Asian (I: 4\%; C: 7\%); other (I: 5\%; C: 5\%)

Socio-economic or socio-demographic status:

- Index of Multiple Deprivation score* (mean \pm SD): I: $17.5 \pm 10.7$; C: $19.3 \pm 10.1$

Interventions

Intervention details (components, length, frequency): participants provided with a home blood pressure monitor, brief training and ongoing nurse-led telephone support targeting BP reduction (average of 3.8 telephone calls over 12 months); participants with consistent blood pressure readings $\geq 130 / 80$ $\mathrm{mmHg}$ advised to consult their GP and received intensified nurse-led telephone follow-up until the target was reached (i.e. implementation of protocols for $B P$ reduction)

Location: community

Mode of delivery: home visits and telephone follow-up

Personnel responsible for delivery: nurse

Timing post-stroke: $\leq 9$ months

Control: baseline assessment conducted during home visit and all participants with $\mathrm{BP}>150 / 90 \mathrm{mmHg}$ were advised to see their GP; usual care provided by GP (all GPs sent information about the study and 
Kerry 2013 (Continued)

a recommended target for home blood pressure of $<130 / 80 \mathrm{mmHg}$ ); participants in the control group received telephone calls after 3 and 9 months to check on their well-being

\begin{tabular}{ll}
\hline Outcomes & 12 months: SBP; DBP, proportion of participants with recurrent stroke \\
\hline General Information & $\begin{array}{l}\text { Funding: the main study was funded by The Stroke Association (grant no. TSA 2006/05). The feasibility } \\
\text { study was funded by The Isaac Schapera Research Trust } \\
\text { Country of origin: UK } \\
\text { Publication language: English } \\
\text { Analysis method: available case analysis } \\
\text { Risk of bias: low } \\
\text { *Trialists state that "the Index of Multiple Deprivation 2007 scale is a measure of poverty and is based } \\
\text { on postal codes and ranges from 0.37 to 85.46. A higher score indicates higher deprivation. Further in- } \\
\text { formation can be found at www.communities.gov.uk/communities/research/indicesdeprivation/depri- } \\
\text { vation10/" }\end{array}$ \\
\hline
\end{tabular}

\section{Risk of bias}

\begin{tabular}{lll}
\hline Bias & Authors' judgement & Support for judgement \\
\hline $\begin{array}{l}\text { Random sequence genera- } \\
\text { tion (selection bias) }\end{array}$ & Low risk & $\begin{array}{l}\text { "A computer-generated randomization sequence to implement stratified ran- } \\
\text { domization ... with a 1:1 allocation using random block sizes of } 4 \text { and 6" }\end{array}$ \\
\hline $\begin{array}{l}\text { Allocation concealment } \\
\text { (selection bias) }\end{array}$ & Low risk & $\begin{array}{l}\text { "Allocation to the intervention or control group was contained within a sealed, } \\
\text { numbered envelope and assigned to the participant by the trial administra- } \\
\text { tor before the baseline visit. The research nurse opened the envelope after she } \\
\text { completed the home baseline assessment." }\end{array}$ \\
\hline
\end{tabular}

Incomplete outcome data Low risk (attrition bias)

All outcomes

Missing data reported by group

Attrition: I: 18/187 (9 died, 5 lost contact, 1 moved away, 3 declined); C: 25/194 (10 died, 6 lost contact, 5 withdrew because of illness, 2 moved away, 2 declined)

Excluded from analysis: I: 1/187 (reason not provided); C: 0

Judgement: reasons for missing data reported and review authors judged that they were unlikely to be related to study outcomes

Selective reporting (re- Low risk Protocol is available and outcomes are reported in the pre-specified way porting bias)

Other bias Low risk The study appears to be free of other sources of bias

Kim 2013

\section{Study characteristics}

Methods RCT

Unit of randomisation: participant

Participants Place of recruitment: neurology clinic


Kim 2013 (Continued)

Numbers randomised: total: 36 (I: $18 ; \mathrm{C}: 18)$

$\%$ Completing final follow-up: $94 \%$

Inclusion criteria: < 12 months since ischaemic stroke; visited a neurology clinic for stroke treatment; normal cognitive function (Mini Mental State Examination > 19); living at home; Internet access

Exclusion criteria: $\mathrm{n} / \mathrm{a}$

Type of stroke (\%): ischaemic (100\%)

Mean age (SD): I: 67.4 (7.3); C: 63.9 (7.4)

Gender (\% men): I: $73 \%$; C: $56 \%$

Ethnicity: not stated

Socio-economic or socio-demographic status (\% graduated the middle school): I: $61 \%$; C: $56 \%$

Interventions

Intervention details (components, length, frequency): 9-week web-based education program focusing on secondary prevention (9 weekly sessions involving video lectures/quizzes, website links to strokerelated information, automated feedback about self-reported health behaviours and the opportunity to email health professionals); guidebook for the programme was provided to participants; research assistant provided telephone-based technical support for the Internet program

Location: participants' homes

Mode of delivery: internet-based education

Personnel responsible for delivery: web-based education program was developed by healthcare professionals

Timing post-stroke: $<12$ months

Control: usual care provided by physicians

\begin{tabular}{ll}
\hline Outcomes & 3 months: total cholesterol, triglycerides, medication adherence \\
\hline General Information & $\begin{array}{l}\text { Funding: this work was supported by Basic Science Research Program through the National Research } \\
\text { Foundation of Korea (NRF) funded by the Ministry of Education, Science and Technology (20110003345) } \\
\text { Country of origin: South Korea } \\
\text { Publication language: English }\end{array}$ \\
\hline
\end{tabular}

$\begin{array}{ll}\text { Notes } & \text { Analysis method: stated intention-to-treat } \\ & \text { Risk of bias: unclear }\end{array}$

\section{Risk of bias}

\begin{tabular}{lll}
\hline Bias & Authors' judgement & Support for judgement \\
\hline $\begin{array}{l}\text { Random sequence genera- } \\
\text { tion (selection bias) }\end{array}$ & Low risk & $\begin{array}{l}\text { "The participants were randomly assigned to an experimental or control group } \\
\text { in a 1:1 ratio, using a computer-generated random code" }\end{array}$ \\
\hline $\begin{array}{l}\text { Allocation concealment } \\
\text { (selection bias) }\end{array}$ & Unclear risk & Not stated \\
\hline $\begin{array}{l}\text { Incomplete outcome data } \\
\text { (attrition bias) } \\
\text { All outcomes }\end{array}$ & Low risk & Missing data reported by group \\
\hline
\end{tabular}


Kim 2013 (Continued)

Judgement: reasons for missing data reported and review authors judged that they were unlikely to be related to study outcomes

\begin{tabular}{lll}
\hline $\begin{array}{l}\text { Selective reporting (re- } \\
\text { porting bias) }\end{array}$ & Unclear risk & Insufficient information (protocol not obtained) \\
\hline Other bias & Low risk & The study appears to be free of other sources of bias \\
\hline
\end{tabular}

Kono 2013

\section{Study characteristics}

\begin{tabular}{ll}
\hline Methods & RCT \\
& Unit of randomisation: participant \\
\hline
\end{tabular}

Participants

Place of recruitment: secondary care

Numbers randomised: total: 70 (I: 35; C: 35$)$

$\%$ Completing final follow-up: $97 \%$

Inclusion criteria: non-cardioembolic stroke confirmed by MRI, ischaemic stroke with large and small vessel diseases, > 20 years old, mRS 0-2 (independent in mobility), discharge directly to home

Exclusion criteria: cardioembolic stroke, cognitive disorders (MMSE < 18), psychiatric disorder, unable to communicate, extracorporeal dialysis hypercoagulable state, lack of motivation to participate

Type of stroke (\%): not stated

Mean age (SD): I: 63.5 (7.0); C: 63.4 (11.4)

Gender (\% men): I: $60 \%$; C: $77.1 \%$

Ethnicity: not stated

Socio-economic or socio-demographic status: not stated counselling about lifestyle modification (increase in physical activity, reduction in salt intake, smoking cessation, alcohol reduction and dietary modification) at baseline, 3 and 6 months. Participants also followed a lifestyle modification program consisting of exercise training and salt restriction once or twice weekly for 24 weeks and a home exercise program

Location: university and home

Mode of delivery: face to face

Personnel responsible for delivery: healthcare interventionist/physical therapists

Timing post-stroke: not stated

Control: participants were provided with advice to facilitate healthy lifestyle modification at baseline 3 and 6 months and the usual medical care

Outcomes 6 months: SBP, LDL, HDL, HbAlc, Waist circumference, BMI, salt intake, physical activity

General Information

Funding: supported by grant-in-aid for challenging exploratory research from the Japan Society for the promotion of science (21650135)

Country of origin: Japan 
Kono 2013 (Continued)

Publication language: English

\begin{tabular}{ll}
\hline Notes & Analysis method: stated intention-to-treat \\
& Risk of bias: unclear
\end{tabular}

\title{
Risk of bias
}

\begin{tabular}{|c|c|c|}
\hline Bias & Authors' judgement & Support for judgement \\
\hline $\begin{array}{l}\text { Random sequence genera- } \\
\text { tion (selection bias) }\end{array}$ & Low risk & $\begin{array}{l}\text { Computer-generated random number sequence using a } 1: 1 \text { basis to lifestyle } \\
\text { modification }\end{array}$ \\
\hline $\begin{array}{l}\text { Allocation concealment } \\
\text { (selection bias) }\end{array}$ & Low risk & Random computer-generated method applied \\
\hline $\begin{array}{l}\text { Incomplete outcome data } \\
\text { (attrition bias) } \\
\text { All outcomes }\end{array}$ & Unclear risk & There is no discussion regarding missing data \\
\hline $\begin{array}{l}\text { Selective reporting (re- } \\
\text { porting bias) }\end{array}$ & Low risk & The study protocol was registered prior to the study initiation \\
\hline Other bias & Unclear risk & $\begin{array}{l}\text { Unclear if recurrent events were presented as number of events rather than } \\
\text { number of people with one or more event }\end{array}$ \\
\hline
\end{tabular}

Kronish 2014

\section{Study characteristics}

\begin{tabular}{ll}
\hline Methods & RCT \\
& Unit of randomisation: participant
\end{tabular}

\section{Participants}

\begin{abstract}
Place of recruitment: senior centres, churches, health fairs, from hospital registries of an academic centre, a federally funded health centre, a home care nursing program, community organisations, through advertising in clinics newspaper adverts
\end{abstract}

Numbers randomised: total: 600 (I: 301; C: 299)

\% Completing final follow-up: I: $80 \%$ C: $89 \%$

Inclusion criteria: stroke or mini stroke within the past 5 years, $\geq 40$ years

Exclusion criteria: lacked capacity to consent, lacked physical or mental capacity to participate meaningfully in workshops, non-English/non-Spanish speaking, institutionalised resident

Type of stroke (\%): ischaemic (100\%)

Mean age (SD): I: 63 (11); C: 64 (11)

Gender (\% women): I: 60\%; C: 59\%

Ethnicity: Black (I: 40\% C: 43\%), Latino (I: 42\% C: 37\%), White (I: 13\% C: 14\%), other (I: 4\% C: 6\%).

Socio-economic or socio-demographic status

- Annual income $\leq 15,000$ dollars/year (\%) (I: 56 C: 58)

- Less than high school education (\%) (I: 31 C: 30$)$ 
Kronish 2014 (Continued) Interventions
Intervention details (components, length, frequency): Weekly peer-led workshops models on chronic disease self-management program. Also received culturally sensitive educational material at randomisation and encouraged to discuss results with a health care provider

Location: community

Mode of delivery: peer-based education

Personnel responsible for delivery: peers

Timing post-stroke: up to 5 years post event

Control: usual care plus the same educational materials at randomisation, a list of local health providers and advice to seek GP. Informed would become involved in intervention after waiting for 1 year

\begin{tabular}{|c|c|}
\hline \multirow[t]{3}{*}{ General Information } & $\begin{array}{l}\text { Funding: funding received from the National Heart, Lung and Blood Institute (K23 HL098359) and the } \\
\text { National Center for Advancing Translational Science (UL1TR000040), the National Institute of Minority } \\
\text { Health and Health Disparities (P60MD00270) and National Center for Research Resources }\end{array}$ \\
\hline & Country of origin: USA \\
\hline & Publication language: English \\
\hline
\end{tabular}

Notes

Risk of bias: unclear risk

\section{Risk of bias}

\begin{tabular}{lll}
\hline Bias & Authors' judgement & Support for judgement \\
\hline $\begin{array}{l}\text { Random sequence genera- } \\
\text { tion (selection bias) }\end{array}$ & Low risk & $\begin{array}{l}\text { Randomisation generated by a computerised random number sequence in } \\
\text { blocks of 2, 4, or } 6\end{array}$ \\
\hline $\begin{array}{l}\text { Allocation concealment } \\
\text { (selection bias) }\end{array}$ & Low risk & Central allocation \\
\hline $\begin{array}{l}\text { Incomplete outcome data } \\
\text { (attrition bias) } \\
\text { All outcomes }\end{array}$ & Low risk & $\begin{array}{l}\text { Missing values were imputed using multiple imputations under the assump- } \\
\text { tion that values were missing at random }\end{array}$ \\
\hline $\begin{array}{l}\text { Selective reporting (re- } \\
\text { porting bias) }\end{array}$ & Unclear risk & Insufficient information to permit judgement \\
\hline \begin{tabular}{l} 
Other bias \\
\hline
\end{tabular} & Low risk & The study appears to be free of other sources of bias \\
\hline
\end{tabular}

\section{Lowe 2007}

\section{Study characteristics}

\begin{tabular}{ll}
\hline Methods & Unit of randomisation: participant \\
\hline Participants & Place of recruitment: hospital stroke unit \\
& Numbers randomised: total: $100 ;$ I: $50 ;$ C: 50
\end{tabular}


Lowe 2007 (Continued)

\author{
$\%$ Completing final follow-up: $84 \%$ \\ Inclusion criteria: stroke; discharged home; able to complete questionnaire or who had carer who \\ could complete questionnaire \\ Exclusion criteria: severe cognitive impairment or communication difficulties; discharged to institu- \\ tional care \\ Type of stroke (\%): ischaemic (I: 96\%; C: 94\%) \\ Median age (IQR): I: 68 (62 to 74$)$; C: 73 (65 to 80 ) \\ Gender (\% men): I: 58\%; C: $62 \%$ \\ Ethnicity: not reported \\ Socio-economic or socio-demographic status: not reported
}

Intervention details (components, length, frequency): information book (CareFile) containing general information about stroke and tailored information about stroke risk factors; researcher explained contents of book to participants/carers during 15 to 20 minute discussion; participants advised to take the CareFile to GP and stroke review clinic appointments

Location: hospital

Mode of delivery: educational materials

Personnel responsible for delivery: researcher (stroke research registrar)

Timing post-stroke: before discharge

Control: usual care ("usual stroke information leaflets (Stroke Association leaflets) provided by the stroke unit and follow-up in a stroke review clinic")

\begin{tabular}{ll}
\hline Outcomes & 3 months and 6 months: SBP; DBP \\
\hline General Information & Funding: the study was supported by a $£ 5000$ research grant from Bristol Myers Squibb \\
& Country of origin: UK \\
& Publication language: English \\
\hline Notes & Analysis method: not stated \\
& Risk of bias: unclear
\end{tabular}

\title{
Risk of bias
}

\begin{tabular}{lll}
\hline Bias & Authors' judgement & Support for judgement \\
\hline $\begin{array}{l}\text { Random sequence genera- } \\
\text { tion (selection bias) }\end{array}$ & Low risk & Shuffling envelopes \\
\hline $\begin{array}{l}\text { Allocation concealment } \\
\text { (selection bias) }\end{array}$ & Low risk & $\begin{array}{l}\text { "When a diagnosis of stroke was confirmed, eligible patients were randomized } \\
\text { by the researcher into the control or intervention group (using sealed opaque } \\
\text { envelopes containing blocks of } 10 \text { names, in a one-to-one ratio)." }\end{array}$ \\
\hline $\begin{array}{l}\text { Incomplete outcome data } \\
\text { (attrition bias) } \\
\text { All outcomes }\end{array}$ & Low risk & $\begin{array}{l}\text { Missing data reported by group } \\
\text { Attrition: I: } 6 / 50 \text { ( } 2 \text { could not be contacted; } 4 \text { died); C: } 10 / 50 \text { (4 could not be } \\
\text { contacted; } 6 \text { died) }\end{array}$
\end{tabular}


Lowe 2007 (Continued)

Judgement: reasons for missing data reported and review authors judged that they were unlikely to be related to study outcomes

\begin{tabular}{lll}
\hline $\begin{array}{l}\text { Selective reporting (re- } \\
\text { porting bias) }\end{array}$ & Unclear risk & Insufficient information (protocol not available) \\
\hline Other bias & Low risk & The study appears to be free of other sources of bias \\
\hline
\end{tabular}

\section{Lowrie 2010}

\section{Study characteristics}

\begin{tabular}{ll}
\hline Methods & RCT \\
Unit of randomisation: general practice \\
\hline
\end{tabular}

Participants

Place of recruitment: 31 general practices

Numbers randomised: total: 4040 (461 stroke/TIA); I: 2373 (289 stroke/TIA); C: 1667 (172 stroke/TIA)

$\%$ Completing final follow-up: information only provided for participant with baseline and follow-up data

Inclusion criteria: previous diagnosis associated with vascular disease ("myocardial infarction, coronary artery bypass graft/angioplasty, angina, angiographic coronary artery disease, stroke/transient ischaemic attack, peripheral ischaemic arterial disease/intermittent claudication or, diabetic patients aged over 45 years")

Type of stroke among participants with a history of stroke/TIA (\%): stroke (66\%); stroke only (56\%); TIA (44\%); TIA only (34\%); stroke and TIA (10\%)

Mean age (SD): I: 68 (11); C: 72 (11)

Gender (\% men): I: 47\%; C: 47\%

Ethnicity: not reported

Socio-economic or socio-demographic status:

- Mean Modified Scottish Index of Multiple Deprivation (SD): I: 46.8 (15.1); C: 35.3 (12.4)

Interventions

Intervention details (components, length, frequency): "pharmacist-led educational outreach directed at general practices, aiming to improve statin prescription for community dwelling patients with vascular disease"; pharmacists received specific training relevant to the delivery of the intervention (5.5 training days); pharmacists delivered 3 educational outreach meetings at each general practice at 4 monthly intervals; pharmacists worked in practices on 1 day per week for 44 weeks to identify participants who were eligible to receive Simvastatin $40 \mathrm{mg}$ and encourage GPs/nurses to systematically contact/follow-up participants

Location: general practices

Mode of delivery: pharmacist-led outreach visits

Personnel responsible for delivery: pharmacists

Timing post-stroke: not reported

Control: practices did not receive pharmacist-led prescribing support 
Lowrie 2010 (Continued)

General Information
Funding: the study was funded and sponsored by NHS Greater Glasgow and Clyde

Country of origin: UK

Publication language: English

Notes Analysis method: $\mathrm{n} / \mathrm{a}$ (available case data used in this review)

Risk of bias: unclear

\section{Risk of bias}

\begin{tabular}{|c|c|c|}
\hline Bias & Authors' judgement & Support for judgement \\
\hline $\begin{array}{l}\text { Random sequence genera- } \\
\text { tion (selection bias) }\end{array}$ & Low risk & "Random number table" \\
\hline $\begin{array}{l}\text { Allocation concealment } \\
\text { (selection bias) }\end{array}$ & Low risk & N/A: all clusters were randomised at once \\
\hline $\begin{array}{l}\text { Incomplete outcome data } \\
\text { (attrition bias) } \\
\text { All outcomes }\end{array}$ & Unclear risk & $\begin{array}{l}\text { Reasons for missing data not available since results were only presented for } \\
\text { participants with baseline and follow-up data (confirmed via correspondence } \\
\text { with trialists) }\end{array}$ \\
\hline $\begin{array}{l}\text { Selective reporting (re- } \\
\text { porting bias) }\end{array}$ & Low risk & Study protocol available and outcomes are reported in the pre-specified way \\
\hline Other bias & Low risk & The study appears to be free of other sources of bias \\
\hline
\end{tabular}

Maasland 2007

\section{Study characteristics}

Methods RCT

Unit of randomisation: participant

Participants Place of recruitment: TIA service ("provides a rapid diagnostic work-up of patients with TIA or minor
stroke in a single day")

Numbers randomised: total: 65 (I: 33; C: 32)

$\%$ Completing final follow-up: $88 \%$

Inclusion criteria: $<3$ months since TIA or minor ischaemic stroke; $\geq 18$ years; fluent in spoken and written Dutch; modified Rankin score $<4$

Exclusion criteria: involved in cardiovascular health education; aphasia, dementia (diagnosis based on DSM-Iv criteria); visual impairment that would affect health education

Type of stroke: TIA (I: 57\%; C: 52\%); minor stroke (I: 43\%; C: 46\%)

Mean age (SD): I: 65 (12); C: 63 (13)

Gender (\% men): I: 57\%; C: 63\%

Ethnicity: not reported

Socio-economic or socio-demographic status: 
- Educational level (\%): I: primary school - 27\%, secondary school - 37\%, college - 20\%, university - 17\%; C: primary school - $15 \%$, secondary school $-41 \%$, college - $26 \%$, university - $19 \%$

Interventions

Intervention details (components, length, frequency): 20 to 25 minute computerised education program about TIA and stroke, antiplatelet and anticoagulant medication and modifiable risk factor control; information tailored according to the impact of each risk factor on secondary prevention (calculated using algorithm) and each patient's current risk factor status, treatment status, educational level and age; participants received a printed summary of the information

Location: TIA service

Mode of delivery: computer-based education

Personnel responsible for delivery: $n / a$

Timing post-stroke: acute TIA or minor stroke

Control: usual care (health education by a neurologist as part of the TIA service)

\begin{tabular}{ll}
\hline Outcomes & $\begin{array}{l}12 \text { weeks: SBP; DBP; total cholesterol; LDL, triglycerides; BMl; compliance with anticoagulants; compli- } \\
\text { ance with lipid-lowering medication; compliance with antihypertensive medication }\end{array}$ \\
\hline General Information & $\begin{array}{l}\text { Funding: this project was funded by the Revolving Fund of the Erasmus Medical Center } \\
\text { Country of origin: Netherlands } \\
\text { Publication language: not stated }\end{array}$ \\
\hline Notes & Analysis method: available case analysis \\
& Risk of bias: low
\end{tabular}

\section{Risk of bias}

\begin{tabular}{|c|c|c|}
\hline Bias & Authors' judgement & Support for judgement \\
\hline $\begin{array}{l}\text { Random sequence genera- } \\
\text { tion (selection bias) }\end{array}$ & Low risk & $\begin{array}{l}\text { "Treatment allocation was random, and based on computer-generated ran- } \\
\text { dom numbers" }\end{array}$ \\
\hline $\begin{array}{l}\text { Allocation concealment } \\
\text { (selection bias) }\end{array}$ & Low risk & $\begin{array}{l}\text { "The randomization was blocked in lots of 10; block size was unknown to the } \\
\text { investigators at the time of the trial" }\end{array}$ \\
\hline \multirow{4}{*}{$\begin{array}{l}\text { Incomplete outcome data } \\
\text { (attrition bias) } \\
\text { All outcomes }\end{array}$} & \multirow[t]{4}{*}{ Low risk } & Missing data reported by group \\
\hline & & Attrition: I: 2/33 lost to follow-up; C: 5/32 lost to follow-up \\
\hline & & Excluded from analysis: I: $1 / 33$ professional health worker (ineligible); C: 0/32 \\
\hline & & $\begin{array}{l}\text { Judgement: reasons for missing data reported and review authors judge that } \\
\text { they are unlikely to be related to study outcomes }\end{array}$ \\
\hline $\begin{array}{l}\text { Selective reporting (re- } \\
\text { porting bias) }\end{array}$ & Low risk & $\begin{array}{l}\text { Protocol available and primary outcomes were reported in the pre-specified } \\
\text { way }\end{array}$ \\
\hline Other bias & Low risk & The study appears to be free of other sources of bias \\
\hline
\end{tabular}

\section{MacKenzie 2013}

\section{Study characteristics}


MacKenzie 2013 (Continued)
Methods
RCT
Unit of randomisation: participant

Place of recruitment: 4 urban stroke prevention
Numbers randomised: total: 56 (I: 29; C: 27$)$
\% Completing final follow-up: $100 \%$
Inclusion criteria: probable TIA or confirmed st
(Montreal Cognitive Score <26) OR < 100\% med
adherence; uncontrolled hypertension (BP > 140/
betes or chronic renal insufficiency
Exclusion criteria: inability to speak/read English;
Type of stroke: stroke (64\%); TIA (36\%)
Age: $>65$ years: $59 \%$
Gender (\% men): $68 \%$
Ethnicity: not reported
Socio-economic or socio-demographic status:
• living alone (21\%);
- education < 9 years (16\%)

Interventions

Intervention details (components, length, frequency): nurse-led intervention targeting participants at high risk of sub-optimal BP control or non-adherence to antihypertensive medication: involved medication counselling, provision of home BP monitoring equipment and medication Dosette, and nurseled telephone calls (monthly intervals for 6 months) to deliver motivational interviewing for secondary prevention behaviours (nurses responsible for delivering the intervention received training in motivational interviewing techniques)

Location: community

Mode of delivery: outpatient appointment and telephone follow-up

Personnel responsible for delivery: nurse practitioner/clinical nurse specialist

Timing post-stroke: not reported

Control: usual care - "stroke physician specialist assessment, initiation and titration of BP medication, adherence and risk factor counselling at clinic visits and follow-up by family physicians"

Outcomes 6 months: stroke recurrence, SBP, DBP, BP < 140/90 mmHg; adherence to antihypertensive medication

General Information Funding: this research was funded by a grant from the Ontario Stroke System (2010-2011)

Country of origin: Canada

Publication language: not stated

Notes Analysis method: intention-to-treat

Risk of bias: low

\section{Risk of bias}


MacKenzie 2013 (Continued)

Random sequence genera- Low risk $\quad$ "Centralized telephone randomization system"
tion (selection bias)

Allocation concealment $\quad$ Low risk
(selection bias)

\begin{tabular}{lll}
\hline $\begin{array}{l}\text { Incomplete outcome data } \\
\text { (attrition bias) } \\
\text { All outcomes }\end{array}$ & Unclear risk & $\begin{array}{l}\text { There was an apparent inconsistency with the standard deviation values re- } \\
\text { ported. Email contact was attempted clarify; however, we did not receive a re- } \\
\text { sponse, so we used the published standard deviation values. }\end{array}$ \\
\hline $\begin{array}{l}\text { Selective reporting (re- } \\
\text { porting bias) }\end{array}$ & Low risk & $\begin{array}{l}\text { Examination of study reports suggests that all outcomes were reported in the } \\
\text { pre-specified way }\end{array}$ \\
\hline Other bias & Low risk & The study appears to be free of other sources of bias \\
\hline
\end{tabular}

Mant 2016

\section{Study characteristics}

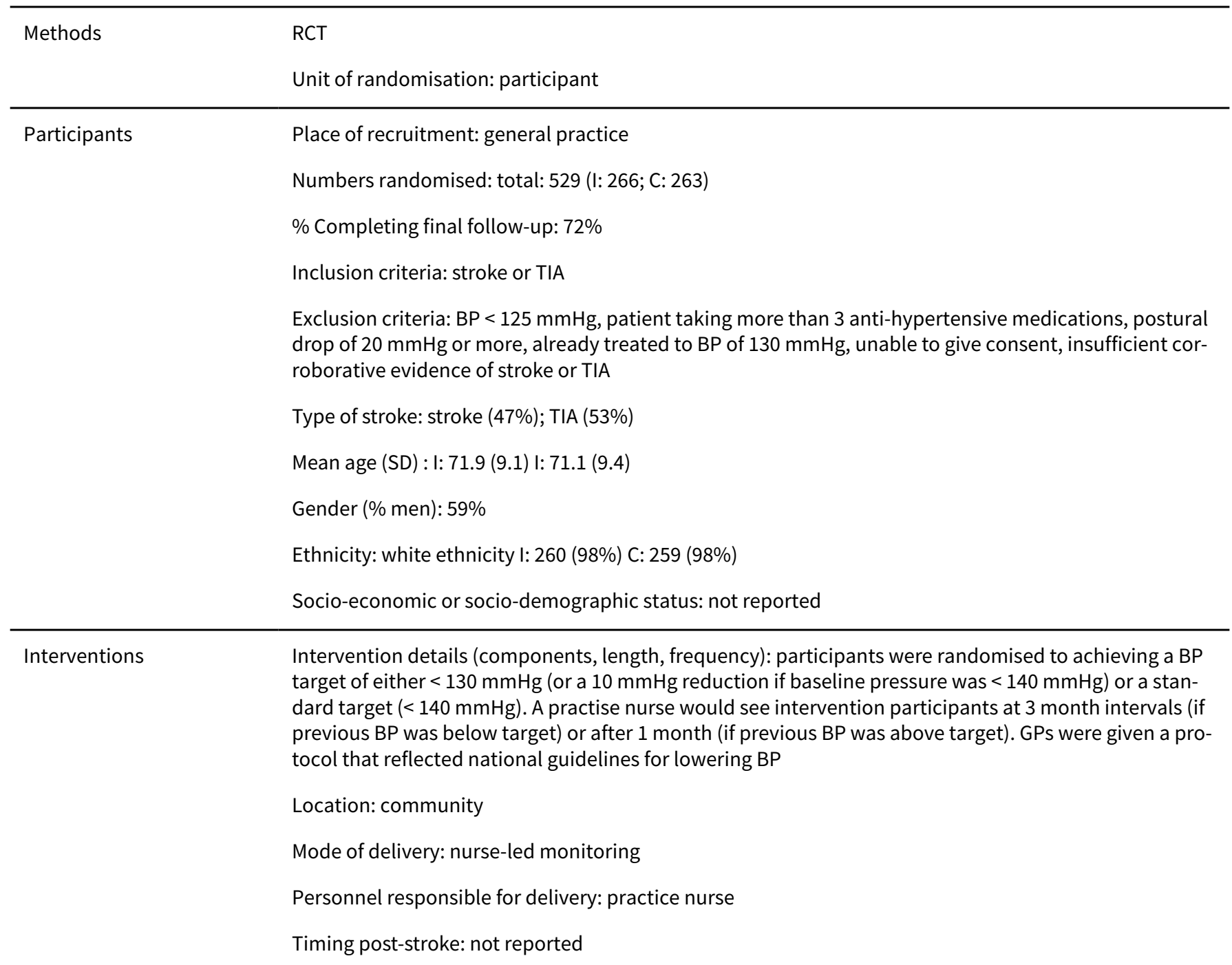


Mant 2016 (Continued)

Control: usual care - whereby the BP target was $<149 \mathrm{mmHG}$, irrespective of baseline BP with the same practice nurse monitoring as the intervention group

\begin{tabular}{|c|c|c|}
\hline Outcomes & \multicolumn{2}{|c|}{ Primary outcome was change in systolic BP between baseline and 1 year } \\
\hline \multirow[t]{3}{*}{ General Information } & \multicolumn{2}{|c|}{$\begin{array}{l}\text { Funding: funded by the National Institute for Health Research (NIHR; Stroke Prevention in Primary } \\
\text { Care, Programme Grant for Applied Research, RP-PG-06061153) and by an NIHR Professorship }\end{array}$} \\
\hline & \multicolumn{2}{|l|}{ Country of origin: UK } \\
\hline & \multicolumn{2}{|c|}{ Publication language: not stated } \\
\hline \multirow[t]{2}{*}{ Notes } & \multicolumn{2}{|c|}{ Analysis method: mixed models, adjusting for baseline BP, age group, sex, diabetes, AF and practice } \\
\hline & \multicolumn{2}{|l|}{ Risk of bias: unclear } \\
\hline \multicolumn{3}{|l|}{ Risk of bias } \\
\hline Bias & Authors' judgement & Support for judgement \\
\hline $\begin{array}{l}\text { Random sequence genera- } \\
\text { tion (selection bias) }\end{array}$ & Low risk & Minimisation based on age, sex, diabetes, AF and baseline BP \\
\hline $\begin{array}{l}\text { Allocation concealment } \\
\text { (selection bias) }\end{array}$ & Unclear risk & Insufficient information to permit judgement \\
\hline $\begin{array}{l}\text { Incomplete outcome data } \\
\text { (attrition bias) } \\
\text { All outcomes }\end{array}$ & Low risk & Missing values were assessed using by three approaches \\
\hline $\begin{array}{l}\text { Selective reporting (re- } \\
\text { porting bias) }\end{array}$ & Low risk & Protocol used has been previously published \\
\hline Other bias & Low risk & The study appears to be free of other sources of bias \\
\hline
\end{tabular}

Markle-Reid 2011

\title{
Study characteristics
}

\begin{tabular}{ll}
\hline Methods & RCT \\
& Unit of randomisation: participant \\
\hline
\end{tabular}

Participants

\author{
Place of recruitment: community care access centre \\ Numbers randomised: total: 101 (I: 52; C: 49) \\ $\%$ Completing final follow-up: $81 \%$ \\ Inclusion criteria: < 18 months since stroke or TIA; living in community; newly referred ( $<2$ weeks) to \\ home care services; competent to give informed consent or substitute decision maker available; com- \\ petent in English or with an interpreter available \\ Type of stroke (\%): not reported \\ Mean age (SD): I: 75.8 (12.4); C: 70.6 (14.5) \\ Gender (\% men): I: 49\%; C: $62 \%$
}


Markle-Reid 2011 (Continued)

Ethnicity: not reported

Socio-economic or socio-demographic status:

- $\operatorname{married}(\%)$ : I: $40 \%$; C: $51 \%$

- living with others (\%): I: 54\%; C: 64\%

Interventions

Intervention details (components, length, frequency): usual home care services plus organised home visits from an inter-professional team (care co-ordinator, nurse, physiotherapist, occupational therapist, speech language pathologist, dietician, social worker, physiotherapist, personal support worker) over a 12-month period; rehabilitation followed evidence-based rehabilitation protocols addressing community reintegration and stroke prevention; use of standardised screening tools e.g. stroke risk assessment tool; members of interdisciplinary team met at monthly case conferences and attended training sessions delivered by the study investigators

Location: community

Mode of delivery: home visits; healthcare provider meetings

Personnel responsible for delivery: inter-professional team

Timing post-stroke: $<18$ months

Control: usual home care services (follow-up by a care coordinator who provided in-home assessments and coordinated home support services)

\section{Outcomes}

General Information

12 months: number of secondary strokes

Funding: this study was supported by grants from the Canadian Institutes of Health Research (CIHR) Institute of Health Services and Policy Research, the CIHR Knowledge Translation Branch (GrantNo.:78692) and the Ontario Ministry of Health and Long-Term Care. Additional funding was provided byMcMaster University System-Linked Research Unit,Toronto Central CCAC, Bridgepoint Health, Ontario Heart and Stroke Foundation, and the GTA Rehabilitation Network

Country of origin: Canada

Publication language: English

\begin{tabular}{ll}
\hline Notes & Analysis method: not stated \\
& Risk of bias: unclear
\end{tabular}

\section{Risk of bias}

\begin{tabular}{lll}
\hline Bias & Authors' judgement & Support for judgement \\
\hline $\begin{array}{l}\text { Random sequence genera- } \\
\text { tion (selection bias) }\end{array}$ & Low risk & $\begin{array}{l}\text { "Randomly generated numbers constructed by a biostatistician who was not } \\
\text { involved in the recruitment process" }\end{array}$ \\
\hline $\begin{array}{l}\text { Allocation concealment } \\
\text { (selection bias) }\end{array}$ & Low risk & "Consecutively numbered, sealed, opaque envelopes" \\
\hline $\begin{array}{l}\text { Incomplete outcome data } \\
\text { (attrition bias) } \\
\text { All outcomes }\end{array}$ & Low risk & $\begin{array}{l}\text { Missing data reported by group } \\
\text { Attrition: I: } 9 / 52 \text { (I: } 4 \text { died; } 4 \text { refused; } 1 \text { unable to contact); C: } 10 / 49 \text { (C: } 3 \text { died; } 7 \\
\text { refused) }\end{array}$ \\
& $\begin{array}{l}\text { Judgement: reasons for missing data reported and review authors judge that } \\
\text { they are unlikely to be related to study outcomes }\end{array}$ \\
\hline
\end{tabular}


Markle-Reid 2011 (Continued)

Selective reporting (re- Unclear risk $\quad$ Insufficient information (protocol not obtained)
porting bias)

Other bias Low risk The study appears to be free of other sources of bias

McAlister 2014

\section{Study characteristics}

\begin{tabular}{ll}
\hline Methods & RCT \\
& Unit of randomisation: participant \\
\hline
\end{tabular}

Place of recruitment: outpatient clinic
Numbers randomised: total: 279 (I: 143; C: 136)
\% Completing final follow-up: $86 \%$
Inclusion criteria: ischaemic stroke or TIA confirmed by a stroke specialist at one of 3 clinics in Edmon-
ton Canada, > 18 years age, systolic BP or LDL cholesterol above guideline-recommended targets (aver-
age systolic BP over 2 visits > $140 \mathrm{mmHg}$, fasting LDL cholesterol > 2.0mmol/L or total: HDL cholesterol
$>4.0)$
Type of stroke (\%): stroke (I: $45.4 \%$ C: $40.4 \%)$, TIA (I: $51.1 \%$ C: $55.9 \%)$, ocular (I: $3.5 \%$ C: $3.7 \%)$
Mean age (SD): I: 68.8 (11.1); C: 66.6 (11.3)
Gender (\% men): I: $60.8 \%$; C: $55.2 \%$
Ethnicity: not reported
Socio-economic or socio-demographic status: not reported

Interventions Intervention details (components, length, frequency): the intervention group was managed by prescribing pharmacists who gave advice on lifestyle (exercise/low salt diet/smoking cessation/medication adherence), checked BP and LDL and initiated or titrated antihypertensive medication and/or lipid lowering therapy

Location: community

Mode of delivery: community

Personnel responsible for delivery: nurse and a prescribing pharmacist

Timing post-stroke: not stated

Control: the intervention group was compared to a group managed by a nurse who gave advice on lifestyle (exercise/low salt diet/smoking cessation/medication adherence), checked BP and LDL and then sent a list of the findings to the patients GP after each visit

Outcomes

Proportion of participants at 6 months who attained optimal blood pressure ( $\leq 140 \mathrm{mmHg}$ systolic BP) and fasting LDL cholesterol $\leq 2.0 \mathrm{mmol} / \mathrm{L}$

General Information Funding: project-specific funding for this trial was provided by the Heart and Stroke Foundation of Alberta, the Alberta Heritage Foundation for Medical Research, and Knowledge Translation Canada

Country of origin: Canada

Publication language: English 
McAlister 2014 (Continued)

Notes $\quad$ Analysis method: intention-to - treat
Risk of bias: low risk

\section{Risk of bias}

\begin{tabular}{|c|c|c|}
\hline Bias & Authors' judgement & Support for judgement \\
\hline $\begin{array}{l}\text { Random sequence genera- } \\
\text { tion (selection bias) }\end{array}$ & Low risk & $\begin{array}{l}\text { Computer generated random numbers with variable sized blocked randomisa- } \\
\text { tion stratified by stroke prevention clinic to preserve allocation concealment }\end{array}$ \\
\hline $\begin{array}{l}\text { Allocation concealment } \\
\text { (selection bias) }\end{array}$ & Low risk & Central allocation \\
\hline $\begin{array}{l}\text { Incomplete outcome data } \\
\text { (attrition bias) } \\
\text { All outcomes }\end{array}$ & Low risk & $\begin{array}{l}\text { Missing data were imputed with a last observation carried forward strategy - } \\
\text { assumed no change in BP or lipid level. Missing data has been imputed using } \\
\text { appropriate methods }\end{array}$ \\
\hline $\begin{array}{l}\text { Selective reporting (re- } \\
\text { porting bias) }\end{array}$ & Low risk & The protocol has been published previously \\
\hline Other bias & Unclear risk & $\begin{array}{l}\text { Unclear if recurrent events were presented as number of events rather than } \\
\text { number of people with one or more event }\end{array}$ \\
\hline
\end{tabular}

\section{McManus 2014}

\section{Study characteristics}

\begin{tabular}{ll}
\hline Methods & RCT \\
& Unit of randomisation: participant \\
\hline
\end{tabular}

Participants

Place of recruitment: general practice patient records

Numbers randomised: total: 555 (I: 277; C: 278)

$\%$ Completing final follow-up: $81 \%$

Inclusion criteria: > 35 years of age, at least 1 high risk conditions (including previous stroke/dia-

betes/stage 3 chronic kidney disease/cardiovascular disease), BP $\geq 130 / 80$

Type of stroke (\%): not reported

Mean age (SD): I: 75.8 (12.4); C: 70.6 (14.5)

Gender (\% men): I: 49\%; C: 62\%

Ethnicity: I: white 96\% C: white 96\%

Socio-economic or socio-demographic status: not given

Interventions

Intervention details: participants were trained how to take their own BP. They were also given a protocol of how to titrate antihypertensive medication. Participants were asked to take their BP twice daily and followed a protocol if not in range

Location: community

Mode of delivery: community 
McManus 2014 (Continued)

Personnel responsible for delivery: not reported

Timing post-stroke: not reported

Control: usual care without any specific BP targets

\begin{tabular}{ll}
\hline Outcomes & BP differences at 1 year for stroke subgroup analysis \\
\hline General Information & $\begin{array}{l}\text { Funding: research funded by the National Institute for Health Research (NIHR) under its Programme } \\
\text { Grants for Applied Research Programme (Grant Reference Number RP-PG 0606-1153), by the NIHR Na- } \\
\text { tional School of Primary Care Research (NSPCR16), and by an NIHR career development fellowship } \\
\text { Country of origin: UK } \\
\text { Publication language: English }\end{array}$ \\
\hline
\end{tabular}

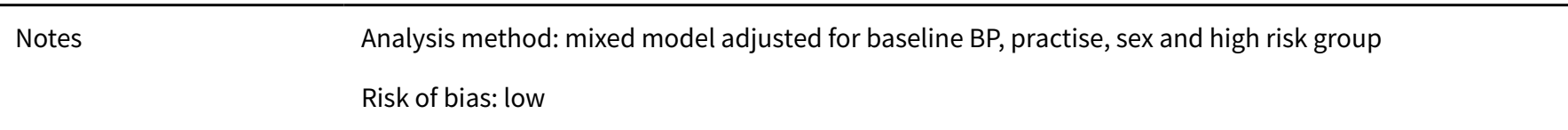

\section{Risk of bias}

\begin{tabular}{lll}
\hline Bias & Authors' judgement & Support for judgement \\
\hline $\begin{array}{l}\text { Random sequence genera- } \\
\text { tion (selection bias) }\end{array}$ & Low risk & $\begin{array}{l}\text { Minimisation used - adaptive stratified sampling that balances different } \\
\text { groups or clinical trials simultaneously }\end{array}$ \\
\hline $\begin{array}{l}\text { Allocation concealment } \\
\text { (selection bias) }\end{array}$ & Low risk & Central allocation \\
\hline $\begin{array}{l}\text { Incomplete outcome data } \\
\text { (attrition bias) } \\
\text { All outcomes }\end{array}$ & Low risk & $\begin{array}{l}\text { Multiple imputations for missing values showed a marginally lower mean dif- } \\
\text { ference in systolic BP. Sensitivity analysis did not show any effect on the pri- } \\
\text { mary outcome }\end{array}$ \\
\hline $\begin{array}{l}\text { Selective reporting (re- } \\
\text { porting bias) }\end{array}$ & Low risk & Based on a previously peer reviewed publication \\
\hline \begin{tabular}{l} 
Other bias \\
\hline
\end{tabular} & Low risk & The study appears to be free of other sources of bias \\
\hline
\end{tabular}

MIST 2014

\section{Study characteristics}

\begin{tabular}{ll}
\hline Methods & RCT \\
& Unit of randomisation: participant \\
\hline Participants & Place of recruitment: inpatient ward \\
& Numbers randomised: total: 386 (I: 193; C: 193) \\
Completing final follow-up: $86 \%$ for systolic BP, $61 \%$ for LDL \\
Inclusion criteria: first ever stroke \\
$\begin{array}{l}\text { Exclusion criteria: impairment precluding participation (e.g. aphasia, psychiatric conditions, cognitive } \\
\text { impairment), unable to converse in English, unable to give consent, other condition likely to affect par- } \\
\text { ticipation (e.g. significant aphasia), receiving psychiatric/psychological treatment, discharged to hospi- } \\
\text { tal/nursing home where medications given by staff or if participation likely to overburden individual }\end{array}$ \\
\hline
\end{tabular}


MIST 2014 (Continued)

Type of stroke: not stated

Mean age (SE): not stated

Gender (\% men): not stated

Ethnicity (\%) : Maori (I: 10.3 C: 7.2 ), Pacific Islander (I: 8.8 C: 4.7), Asian (I: $2.1 \%$ C: $4.7 \%$ ), New Zealand European/other (I: $78.8 \mathrm{C}: 83.4$ )

Socio-economic or socio-demographic status (\%):

- marital status: married/civil union/de facto (I: 69.9 C: 72.5 ), never married (I: 4.7 C: 5.2), separated/divorced/widowed (I: 25.4 C: 22.3 )

- prior living situation: living with family (I: 73.1 C 76.7), living with others (I: 3.1 C: 4.1 ), living alone (I: 23.8 C: 19.2)

- prior dwelling place: own home (I: 64.2 C: 73.1), rented (I: 20.7 C: 16.1), living with family/friends (I: 5.2 C 3.1), retirement village/similar (I: 8.3 C: 5.2 ), rest home/private hospital (I: 0.5 C: 0.5 ), other (I: 1 C: 0.5 ), missing (I: 0 C: 0.5 )

- completed high school: yes (I: 80.3 C: 82.4), no (I: 19.2 C: 17.1), missing (I: 0.5 C: 0.5 )

- highest further qualification: degree (I: $17.6 \mathrm{C}: 21.8)$, diploma/certificate (I: $17.1 \mathrm{C}: 21.8)$, trade/technical (I: 16.1 C: 14), other (I:3.1 C: 6.2), missing (I: 46.1 C: 36.3 )

- employment type: professional (I: $7.8 \mathrm{C}: 9.3)$, manager/technical (I: $18.1 \mathrm{C}: 19.7)$, skilled non-manual (I: 10.4 C: 4.7), skilled manual (I: 8.8 C: 8.3), partly skilled (I: 3.6 C: 3.1), unskilled (I: 5.2 C: 4.1), armed forces (I: 0.5 C: 0.5 )

Interventions

Intervention details: usual care, in addition to 4 motivational interviewing sessions (at 28 days, 3,6 and 9 months post stroke) - the first session was face-to-face either in the participant's home or in hospital and then a further 3 by telephone or face-to-face if telephone was not possible. A letter was sent to the participant's GP to remind them of the participant's participation and a reminder of recommendations to monitor BP and lipid

Location: secondary care/community

Mode of delivery: face-to-face and/or telephone follow-up

Personnel responsible for delivery: researcher

Timing post-stroke: started at 28 days post stroke

Control: after discharge, participants were followed up by their GP or designated stroke centre every 3 to 6 months as part of the usual stroke care

Outcomes

Self-reported medication adherence at 3, 6 and 9 months; systolic BP at 12 months; LDL, HDL and total cholesterol at 12 months

General Information Funding: funded by the New Zealand Health Research Council (HRC Ref 10/458)

Country of origin: New Zealand

Publication language: English

Notes Analysis method: stated intention-to-treat

Risk of bias: low

\section{Risk of bias}

Bias Authors' judgement Support for judgement

Random sequence genera- Low risk

A randomisation technique from a previously published protocol was used tion (selection bias) 
MIST 2014 (Continued)

Allocation concealment $\quad$ Low risk
(selection bias)

Incomplete outcome data Low risk

(attrition bias)

Sensitivity analysis was undertaken. Missing data on the primary outcome was

All outcomes

\begin{tabular}{lll}
\hline $\begin{array}{l}\text { Selective reporting (re- } \\
\text { porting bias) }\end{array}$ & Low risk & The study protocol was published within a previously peer reviewed journal \\
\hline Other bias & Low risk & The study appears to be free of other sources of bias \\
\hline
\end{tabular}

Nailed Stroke 2010

\section{Study characteristics}

\begin{tabular}{ll}
\hline Methods & RCT \\
& Unit of randomisation: participant \\
\hline
\end{tabular}

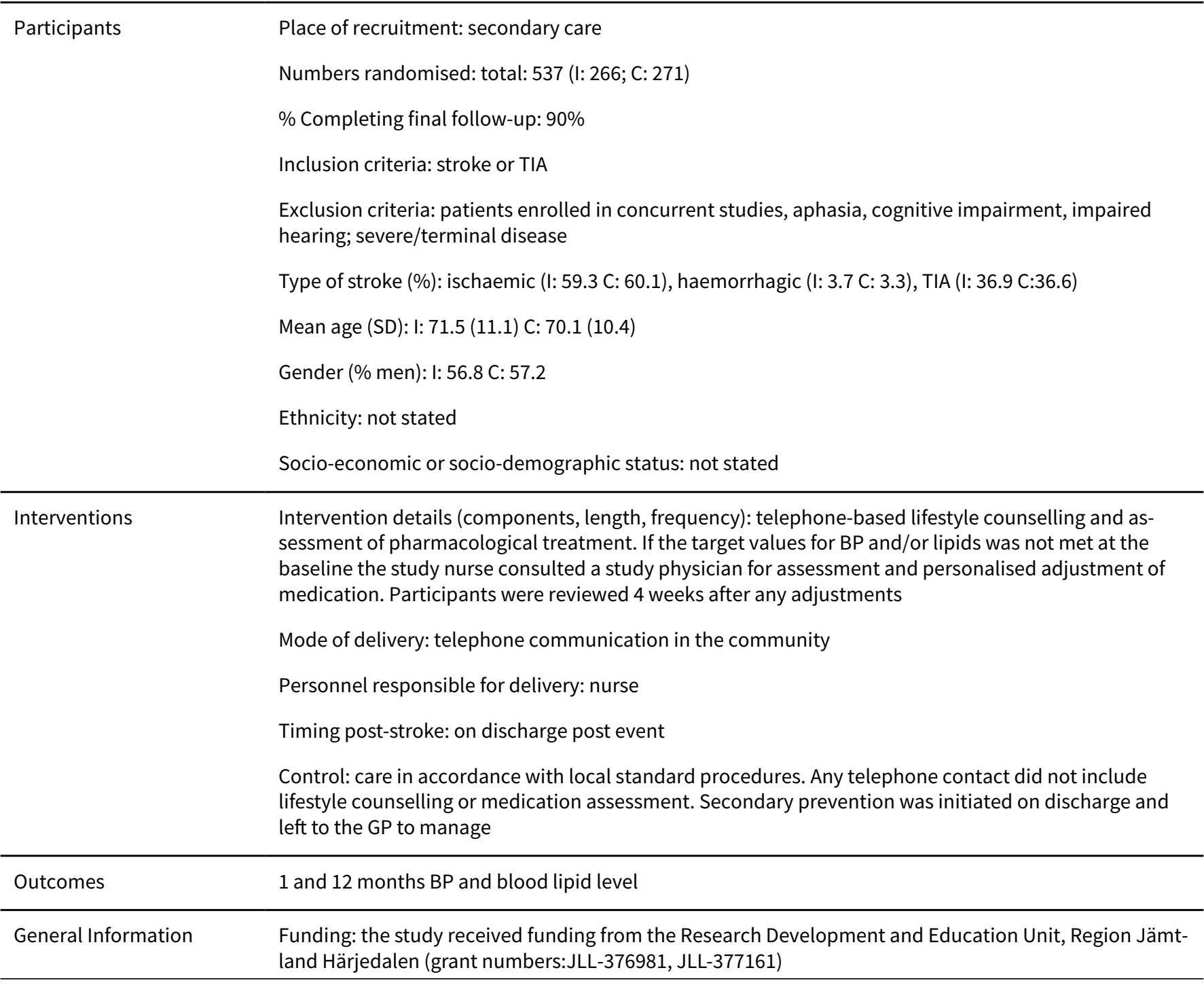


Nailed Stroke 2010 (Continued)

Country of origin: Sweden

Publication language: English

\begin{tabular}{ll}
\hline Notes & Analysis method: stated intention-to-treat \\
& Risk of bias: unclear \\
\hline
\end{tabular}

\section{Risk of bias}

\begin{tabular}{lll}
\hline Bias & Authors' judgement & Support for judgement \\
\hline $\begin{array}{l}\text { Random sequence genera- } \\
\text { tion (selection bias) }\end{array}$ & Low risk & $\begin{array}{l}\text { Computer generated randomisation in blocks of 4, stratified for sex and de- } \\
\text { gree of disability. 2 parallel groups were compared - allocation ration of 1:1 }\end{array}$ \\
\hline $\begin{array}{l}\text { Allocation concealment } \\
\text { (selection bias) }\end{array}$ & Low risk & Robust method for allocation described \\
\hline $\begin{array}{l}\text { Incomplete outcome data } \\
\text { (attrition bias) } \\
\text { All outcomes }\end{array}$ & Unclear risk & $\begin{array}{l}\text { Missing data not inputted - however unlikely to be related to the outcome, } \\
\text { hence risk is unclear }\end{array}$ \\
\hline $\begin{array}{l}\text { Selective reporting (re- } \\
\text { porting bias) }\end{array}$ & Low risk & \begin{tabular}{l} 
Study protocol has been published and was available before the study \\
\hline Other bias
\end{tabular} \\
\hline
\end{tabular}

O'Carroll 2011

\section{Study characteristics}

\begin{tabular}{ll}
\hline Methods & RCT \\
& Unit of randomisation: participant
\end{tabular}

\section{Participants}

\section{Place of recruitment: hospital stroke clinic and stroke unit}

Numbers randomised: total: 62 (I: 31; C:31)

$\%$ Completing final follow-up: $87 \%$

Inclusion criteria: first stroke or TIA; discharged home; prescribed secondary prevention antihypertensive medication; sub-optimal medication adherence score

Exclusion criteria: requirement for help with taking medications; using a Dosette box; cognitive difficulties that precluded participation in the study

Type of stroke (\%): not reported

Mean age (SD): I: 68 (11); C: 71 (11)

Gender (\% men): 65\%

Ethnicity: not reported

Socio-economic or socio-demographic status (Scottish Index of Multiple Deprivation Quintile): 1 (highest deprivation) - 2\%, 2 - 10\%, 3 - 19\%, 4 - 19\%, 5 (lowest deprivation) - 51\% 
O'Carroll 2011 (Continued)

Interventions
Intervention details (components, length, frequency): 2 intervention sessions (approximately 30 minutes each) conducted 2 weeks apart: session 1 helped participants to establish a better medication-taking routine through completing individualised worksheets; session 2 reviewed participants' plans and addressed barriers to implementation; electronic recording of pill-taking for a duration of 3 months (researcher made monthly home visits to refill the electronic pill bottle)

Location: participants' homes or a research facility

Mode of delivery: home visits

Personnel responsible for delivery: researcher

Timing post-stroke: $<3$ months post-discharge

Control: participants attended 2 sessions with a researcher who "engaged the patient in non-medication related conversation in an attempt to provide some control for non-specific effects of attention/social contact"; electronic recording of pill-taking for 3 months

\begin{tabular}{|c|c|c|}
\hline Outcomes & \multicolumn{2}{|c|}{3 months: medication adherence; SBP; DBP } \\
\hline \multirow[t]{3}{*}{ General Information } & \multicolumn{2}{|c|}{ Funding: this project was funded by a grant from the Scottish Government, Department of Health } \\
\hline & \multicolumn{2}{|l|}{ Country of origin: UK } \\
\hline & \multicolumn{2}{|c|}{ Publication language: English } \\
\hline \multirow[t]{2}{*}{ Notes } & \multicolumn{2}{|c|}{ Analysis method: stated intention-to-treat } \\
\hline & \multicolumn{2}{|l|}{ Risk of bias: low } \\
\hline \multicolumn{3}{|l|}{ Risk of bias } \\
\hline Bias & Authors' judgement & Support for judgement \\
\hline $\begin{array}{l}\text { Random sequence genera- } \\
\text { tion (selection bias) }\end{array}$ & Low risk & $\begin{array}{l}\text { "Participants were randomized to either the Intervention or Control group us- } \\
\text { ing web-based software set up by the Edinburgh Clinical Trials Unit." }\end{array}$ \\
\hline $\begin{array}{l}\text { Allocation concealment } \\
\text { (selection bias) }\end{array}$ & Low risk & Web-based randomisation \\
\hline \multirow{4}{*}{$\begin{array}{l}\text { Incomplete outcome data } \\
\text { (attrition bias) } \\
\text { All outcomes }\end{array}$} & \multirow[t]{4}{*}{ Low risk } & Missing data reported by group \\
\hline & & $\begin{array}{l}\text { Attrition I: } 2 / 31 \text { ( } 2 \text { hospitalised for non-stroke reasons); C: } 2 / 31 \text { ( } 1 \text { hospitalised } \\
\text { for non-stroke reasons; } 1 \text { relocated) }\end{array}$ \\
\hline & & $\begin{array}{l}\text { Excluded from the analysis: (did not receive intervention): I: } 2 / 31 \text { ( } 1 \text { declined to } \\
\text { use electronic pill bottle; } 1 \text { hospitalised for non-stroke reasons); C: } 2 / 31 \text { ( } 2 \text { hos- } \\
\text { pitalised for non-stroke reasons) }\end{array}$ \\
\hline & & $\begin{array}{l}\text { Judgement: reasons for missing data reported and review authors judge that } \\
\text { they are unlikely to be related to study outcomes }\end{array}$ \\
\hline $\begin{array}{l}\text { Selective reporting (re- } \\
\text { porting bias) }\end{array}$ & Low risk & Protocol available and outcomes reported in the pre-specified way \\
\hline Other bias & Low risk & The study appears to be free of other sources of bias \\
\hline
\end{tabular}


Peng 2014

\section{Study characteristics}

\begin{tabular}{|c|c|}
\hline Methods & $\begin{array}{l}\text { RCT } \\
\text { Unit of randomisation: hospital }\end{array}$ \\
\hline Participants & $\begin{array}{l}\text { Place of recruitment: hospital } \\
\text { Numbers randomised: total - participants 3821; I: } 1795 ; \text { C:2026, hospitals I: } 23 ; \text { C: } 24 \\
\text { Completing final follow-up: } 1 \text { hospital withdrew before the study began } \\
\text { Inclusion criteria: > } 18 \text { years old, proven ischaemic stroke confirmed by CT or MRI, TIA, hospitalisation } \\
\text { within } 30 \text { days after the index event; clinical stability, independence in daily activities } \\
\text { Exclusion criteria: CT/MRI evidence of intracerebral haemorrhage, stroke/TIA unrelated to atherosclero- } \\
\text { sis, severe co-morbid illness/unstable medical condition, significant memory/behavioural disorders re- } \\
\text { quiring daily care, concurrent participation in another clinical trial, pregnancy } \\
\text { Type of stroke (\%): not reported } \\
\text { Mean age (SD): I: } 61.48 \text { (11.47); C: } 60.36 \text { (11.66) } \\
\text { Gender (\% men): I: } 67 \text { C: } 69 \\
\text { Ethnicity: not reported } \\
\text { Socio-economic or socio-demographic status: not reported }\end{array}$ \\
\hline
\end{tabular}

Interventions Intervention details (components, length, frequency): the intervention consisted of lifestyle modification with the patients, including smoking cessation, healthy diet, and regular exercise. Patient education included an interactive website based education session emphasising the importance of adhering to the SMART program including information discussing risk-factor control through medication and lifestyle changes

Location: outpatient

Mode of delivery: outpatient and online

Personnel responsible for delivery: clinical researcher

Timing post-stroke: within 30 days

Control: participants "received only those interventions chosen by their attending neurologist-clinician, without the use of the algorithm or interactive education and access to the educational website"

\begin{tabular}{ll}
\hline Outcomes & 12 months: medication adherence \\
\hline General Information & $\begin{array}{l}\text { Funding: funded was provided by the National Key Technology Research and Development Program in } \\
\text { the 11th 5-year plan of China } \\
\text { Country of origin: China } \\
\text { Publication language: English and Chinese }\end{array}$ \\
\hline Notes & Analysis method: linear regression model \\
& Risk of bias: Unclear \\
\hline
\end{tabular}

\section{Risk of bias}

Bias Authors' judgement Support for judgement


Peng 2014 (Continued)

Random sequence genera- Low risk Simple cluster sampling method applied
tion (selection bias)

Allocation concealment High risk
(selection bias)

ion bias)

Incomplete outcome data Unclear risk Does not discuss missing data
(attrition bias)

All outcomes

Selective reporting (re- Low risk Study protocol has been published in a previous publication
porting bias)

\begin{tabular}{ll}
\hline Other bias $\quad$ Unclear risk & $\begin{array}{l}\text { Unclear if recurrent events were presented as number of events rather than } \\
\text { number of people with one or more event }\end{array}$ \\
\hline
\end{tabular}

Pergola 2014

\section{Study characteristics}

\begin{tabular}{ll}
\hline Methods & RCT \\
& Unit of randomisation: participant \\
\hline
\end{tabular}

Place of recruitment: not documented
Numbers randomised: total: 3020 (I: 1501; C: 1519)
\% Completing final follow-up: $98 \%$
Inclusion criteria: lacunar stroke syndrome confirmed by MRI, > 30 years old, normotensive and hyper-
tensive patients
Exclusion criteria: no surgical amenable ipsilateral carotid artery disease, no major risk cardio-embolic
sources
Type of stroke (\%): small subcortical stroke (100\%)
Mean age: $63+/-11$ years
Gender (men): $63 \%$
Ethnicity: white (51\%), Hispanic (30\%), black 916\%)
Socio-economic or socio-demographic status: USA (56\%), Latin America (23\%), Spain (12\%) Canada
(9\%)

Interventions

Intervention details (components, length, frequency): participants were randomised to 1 or 2 levels of BP control either 'intensive' (<130 mm Hg) or 'usual' (130-149 mm Hg). Also participants were randomly assigned to take clopidogrel $75 \mathrm{mg}$ daily or the matching placebo

Location: outpatient clinic

Mode of delivery: outpatient clinic face to face, free prescriptions were given

Personnel responsible for delivery: physicians

Timing post-stroke: 6 months or less 
Pergola 2014 (Continued)

Control: usual care - including standard (<140 mmHg) blood pressure control

Outcomes $\quad 3$ years: time to first stroke relapse; stroke relapse rate; proportion of participants meeting targets for blood pressure, blood fats, blood sugar and BMI

\begin{tabular}{|c|c|}
\hline General Information & $\begin{array}{l}\text { Funding: this research was funded by the National Institute of Neurological Disorders and Stroke } \\
\text { (NINDS \# } 2 \text { U01 NS38529-04A1) } \\
\text { Country of origin: USA } \\
\text { Publication language: English }\end{array}$ \\
\hline Notes & $\begin{array}{l}\text { Analysis method: analysis of variance } \\
\text { Risk of bias: unclear }\end{array}$ \\
\hline
\end{tabular}

\section{Risk of bias}

\begin{tabular}{lll}
\hline Bias & Authors' judgement & Support for judgement \\
\hline $\begin{array}{l}\text { Random sequence genera- } \\
\text { tion (selection bias) }\end{array}$ & Low risk & $\begin{array}{l}\text { Randomised using a 2 x 2 factorial design stratified by clinical centre and base- } \\
\text { line hypertensive stats. Data was inputted and a computer generated unique } \\
\text { number was given to assign participants }\end{array}$ \\
\hline $\begin{array}{l}\text { Allocation concealment } \\
\text { (selection bias) }\end{array}$ & Unclear risk & Insufficient information \\
\hline $\begin{array}{l}\text { Incomplete outcome data } \\
\text { (attrition bias) } \\
\text { All outcomes }\end{array}$ & Unclear risk & Not addressed \\
\hline
\end{tabular}

Selective reporting (re- Low risk Study protocol is available in a previous publication

porting bias)

Other bias Low risk The study appears to be free of other sources of bias

Ranta 2015

\section{Study characteristics}

\begin{tabular}{ll}
\hline Methods & RCT
\end{tabular}

Unit of randomisation: primary care practice/clinic

Participants

Place of recruitment: from local directories - participants were selected if GP practices were involved

Numbers randomised: total: 56 (I: 29; C: 27)

$\%$ Completing final follow-up: $100 \%$

Inclusion criteria: any TIA or stroke, never been exposed to this tool before, access to an organised TIA pathway consistent with the New Zealand TIA guideline

Exclusion criteria: did not present to a participating primary or secondary health care providers during the study period or presented without neurologic/ophthalmologic symptoms

Mean age years (SD): I: 69.8 (13.3) C: 72.3 (14.0)

Gender (men): I: 67, C: 55 
Ranta 2015 (Continued)

Ethnicity: I: European 156/172; C: European 101/119

Interventions Intervention details (components, length, frequency): the tool is a Web-based software program accessed via a GP computer desktop icon. Clicking the icon opens a single page of tick boxes asking for relevant aspects of the presenting illness. Depending on diagnosis and risk estimation, the tool recommends a guideline-based management strategy.

Location: primary care practice/clinic

Mode of delivery: face-to-face

Personnel responsible for delivery: primary care doctor

Timing post-stroke: after initial event

Control: usual care

\begin{tabular}{ll}
\hline Outcomes & Stroke at 90 days, stroke and TIA at 90 days or vascular event/death \\
\hline General Information & Funding: the New Zealand Health Research Council funded this trial \\
& Country of origin: New Zealand \\
& Publication language: English \\
\hline Notes & Analysis method: generalised linear models \\
& Risk of bias: unclear
\end{tabular}

\section{Risk of bias}

\begin{tabular}{lll}
\hline Bias & Authors' judgement & Support for judgement \\
\hline $\begin{array}{l}\text { Random sequence genera- } \\
\text { tion (selection bias) }\end{array}$ & Low risk & $\begin{array}{l}\text { The clusters were general practices randomised one-to-one to intervention } \\
\text { and control groups using a computer-generated simple randomisation sched- } \\
\text { ule }\end{array}$ \\
\hline $\begin{array}{l}\text { Allocation concealment } \\
\text { (selection bias) }\end{array}$ & Low risk & Central allocation \\
\hline $\begin{array}{l}\text { Incomplete outcome data } \\
\text { (attrition bias) } \\
\text { All outcomes }\end{array}$ & Unclear risk & $\begin{array}{l}\text { Low number of GP practices agreed to join in the study and none were exclud- } \\
\text { ed }\end{array}$ \\
\hline $\begin{array}{l}\text { Selective reporting (re- } \\
\text { porting bias) }\end{array}$ & Low risk & Outcomes were recorded electronically by individual GPs/from GP records \\
\hline \begin{tabular}{l} 
Other bias \\
\hline
\end{tabular} & Low risk & The study appears to be free of other sources of bias \\
\hline
\end{tabular}

Slark 2013

\section{Study characteristics}

\begin{tabular}{ll}
\hline Methods & RCT \\
& Unit of randomisation: participant \\
\hline Participants & Place of recruitment: hospital (inpatient) \\
\hline
\end{tabular}


Numbers randomised: total: 96 (I: 47; C: 49)

$\%$ Completing final follow-up: $98 \%$

Inclusion criteria: ischaemic stroke

Exclusion criteria: cognitive or memory difficulties that precluded participation in the intervention

Type of stroke: ischaemic (100\%)

Mean age (SD): I: 65 (12); C: 66 (13)

Gender: I: 64\%; C: 53\%

Ethnicity: White: I: 62\%; C: 67\%; "Black Ethnic Minority (BME) groups made up 13\% of the total cohort"

Socio-economic or socio-demographic status:

- university education: I: 40\%; C: $18 \%$

- married: $57 \%$; C: $55 \%$

Interventions

Intervention details (components, length, frequency): 30-minute risk awareness session: involved tailored information provision on the topics of stroke aetiology, risk factors and secondary prevention medications; participants were informed of their individual risk scores for secondary stroke

Location: hospital

Mode of delivery: inpatient appointment

Personnel responsible for delivery: researcher

Timing post-stroke: initiated prior to hospital discharge

Control: usual care (no additional risk awareness information)

\begin{tabular}{ll}
\hline Outcomes & $\begin{array}{l}3 \text { months: recurrent stroke; SBP, DBP, total cholesterol, adherence to secondary prevention medica- } \\
\text { tions }\end{array}$ \\
\hline General Information & $\begin{array}{l}\text { Funding: this research received no specific grant from any funding agency in the public, commercial or } \\
\text { not-for-profit sectors } \\
\text { Country of origin: UK } \\
\text { Publication language: English }\end{array}$ \\
\hline Notes & Analysis method: available case analysis \\
& Risk of bias: low \\
\hline
\end{tabular}

\section{Risk of bias}

Bias Authors' judgement Support for judgement

Random sequence genera- Low risk "Subjects were randomized using computer-generated random codes" tion (selection bias)

$\begin{array}{ll}\begin{array}{l}\text { Allocation concealment } \quad \text { Low risk } \\ \text { (selection bias) }\end{array} & \text { "The researcher was blind to randomization until after recruitment of each } \\ & \text { participant to avoid selection bias....this was achieved through sealing each } \\ & \text { random code in an envelope prior to commencing the trial, which was only se- } \\ & \text { lected after the participant had been recruited." }\end{array}$

\section{Incomplete outcome data Low risk}

(attrition bias)

All outcomes

\section{Missing data reported by group}

Attrition: I: 0/47; C: 2/47 (2 lost to follow-up) 
Judgement: reasons for missing data reported and review authors judge that they are unlikely to be related to study outcomes

\begin{tabular}{lll}
\hline $\begin{array}{l}\text { Selective reporting (re- } \\
\text { porting bias) }\end{array}$ & Low risk & $\begin{array}{l}\text { Examination of study reports suggests that all outcomes were reported in the } \\
\text { pre-specified way }\end{array}$ \\
\hline Other bias & Low risk & The study appears to be free of other sources of bias \\
\hline
\end{tabular}

\section{Wan 2016}

\section{Study characteristics}

\begin{tabular}{ll}
\hline Methods & RCT \\
& Unit of randomisation: participant \\
\hline
\end{tabular}

Participants

Place of recruitment: neurology department

Numbers randomised: total: 80 (I: 40; C: 40)

$\%$ Completing final follow-up: $100 \%$

Inclusion criteria: ischaemic stroke, > 35 years of age, hospitalised within 1 month of an ischaemic stroke diagnosed by CT/MRI, previously independent with activities of daily living

Exclusion criteria: a history of cardio-embolic infarction, Wernicke's aphasia, cognitive impairment, a history of severe liver or kidney disease, and any known malignancy or other neurological diseases

Type of stroke (\%): ischaemic stroke

Mean age (SD): I: $59.01 \pm 12.36$; C: $60.24 \pm 12.57$

Gender (\% men): I: $75 \%$; C: $67.5 \%$

Ethnicity: not reported

Socio-economic or socio-demographic status: education level, elementary C: $22.5 \%$ I: 22.5 ; middle school C: $27.5 \%$; I: 20\%, high school C: $27.5 \%$; I:27.5\%, undergraduate/graduate school C: $22.5 \%$ I:30\%. Employed C: $25 \%$; I:32.5\%, unemployed C: $27.5 \%$; I: 25\%, retired C:47.5\%; I:42.5\% ed of goal setting advice focused on selected areas with motivational elements. Delivered at 1 week, 1 and 3 months post discharge lasting 15-20 minutes

Location: community

Mode of delivery: telephone

Personnel responsible for delivery: stroke nurse

Timing post-stroke: post hospital discharge

Control: usual care including freely available educational brochures on understanding stroke and reducing stroke risk, in addition to GP follow-up

Outcomes Medication adherence at 3 and 6 months

General Information

Funding: this is a doctoral dissertation and was supported by grants from the Department of Health of Guangdong Province, China (No. A2014211) to Li-Hong Wan, PI. This work was also funded by provincial 
Wan 2016 (Continued)

(Guangdong Science and Technology Department, the Guangdong special program for scientific development, No. 2016A020215039) programs, Li-Hong Wan, PI

Country of origin: China

Publication language: English

Notes Analysis method: analysis of variance

Risk of bias: low

\section{Risk of bias}

\section{Bias}

\section{Authors' judgement Support for judgement}

Random sequence genera- Low risk tion (selection bias)

Parallel group RCT 1:1 group allocation determine by a sealed opaque envelope with a serial number on the outside

\begin{tabular}{lll}
\hline $\begin{array}{l}\text { Allocation concealment } \\
\text { (selection bias) }\end{array}$ & Low risk & Central allocation \\
\hline $\begin{array}{l}\text { Incomplete outcome data } \\
\text { (attrition bias) } \\
\text { All outcomes }\end{array}$ & Low risk & Missing data reported by group \\
\hline $\begin{array}{l}\text { Selective reporting (re- } \\
\text { porting bias) }\end{array}$ & Low risk & $\begin{array}{l}\text { Examination of study reports suggests that all outcomes were reported in the } \\
\text { pre-specified way }\end{array}$ \\
\hline Other bias & Low risk & The study appears to be free of other sources of bias \\
\hline
\end{tabular}

Wang 2005

\section{Study characteristics}

\begin{tabular}{ll}
\hline Methods & RCT \\
& Unit of randomisation: participant \\
\hline Participants & Place of recruitment: hospital \\
& Numbers randomised: total: 198 (I: 146; C: 52$)$ \\
& $\%$ Completing final follow-up: unknown \\
& Inclusion criteria: stroke in internal carotid artery; first stroke \\
& Exclusion criteria: none stated \\
& Type of stroke (\%): not stated \\
& Mean age (SD): I: $63.24 \pm 7.35 ;$ C: $60.94 \pm 9.87$ \\
& Gender (\% men): I: $54 \%$; : $50 \%$ \\
& Ethnicity: not reported \\
& Socio-economic or socio-demographic status: not reported
\end{tabular}


Wang 2005 (Continued)

Interventions

Intervention details (components, length, frequency): follow-up by a neurologist within one week postdischarge and then every at 1, 2 or 3 months; patients and caregivers educated about nursing care, home rehabilitation, neuropsychology and modifiable risk factors

Location: community

Mode of delivery: visits, lectures, leaflets, multimedia teaching

Personnel responsible for delivery: neurologists

Timing post-stroke: $<1$ week post-discharge

Control: usual care

Outcomes 3 years: time to first stroke relapse; stroke relapse rate; proportion of participants meeting targets for
blood pressure, blood fats, blood sugar and BMI

General Information

Funding: this study was supported by the grants from the Ministry of Science and Technology of the People's Republic of China (2011BAI08B02, 2012ZX09303, and 2013BAI09B03), Beijing Institute for Brain Disorders (BIBD-PXM2013_014226_07_000084)

Country of origin: China

Publication language: English

Notes Analysis method: not stated

Risk of bias: low

\section{Risk of bias}

\begin{tabular}{lll}
\hline Bias & Authors' judgement & Support for judgement \\
\hline $\begin{array}{l}\text { Random sequence genera- } \\
\text { tion (selection bias) }\end{array}$ & High risk & $\begin{array}{l}\text { Not stated } \\
\text { Unexplained imbalances in numbers allocated to intervention and control } \\
\text { groups }\end{array}$ \\
\hline $\begin{array}{l}\text { Allocation concealment } \\
\text { (selection bias) }\end{array}$ & Unclear risk & Not stated \\
\hline $\begin{array}{l}\text { Incomplete outcome data } \\
\text { (attrition bias) } \\
\text { All outcomes }\end{array}$ & Unclear risk & Not stated \\
\hline $\begin{array}{l}\text { Selective reporting (re- } \\
\text { porting bias) }\end{array}$ & Unclear risk & No protocol available \\
\hline \begin{tabular}{l} 
Other bias \\
\hline
\end{tabular} & Low risk & The study appears to be free from other sources of bias \\
\hline
\end{tabular}

Welin 2010

\section{Study characteristics}

\begin{tabular}{ll}
\hline Methods & RCT \\
& Unit of randomisation: participant
\end{tabular}


Welin 2010 (Continued)

Participants
Place of recruitment: rural hospital

Numbers randomised: total: 163 (I: 81; C: 82)

$\%$ Completing final follow-up: $71 \%$

Inclusion criteria: ischaemic or haemorrhagic stroke; first stroke; < 85 years; living at home before the stroke

Exclusion criteria: previous stroke; severe dementia; severe stroke (Rankin score > 5); severe cardiovascular disease; life expectancy $<1$ year

Type of stroke (\%): haemorrhagic l:9\%, C:16\%

Mean age (SD): I: 71.2 (9.9); C: 69.6 (11.7)

Gender (\% women): I: 41\%; C: $37 \%$

Ethnicity: not reported

Socio-economic or socio-demographic status: not reported

Intervention: follow-up appointments with a stroke nurse at 1.5, 6 and 12 months post-discharge (included assessment of handicap and depression, measurement of blood pressure, provision of health information and referral to physiotherapist or occupational therapist if necessary); appointments with a stroke physician at 3 and 9 months (included a review of medication and medical problems with referral to other specialists if necessary)

Location: hospital stroke clinic

Mode of delivery: outpatient appointment

Personnel responsible for delivery: stroke nurse and stroke physician

Timing post-stroke: 1.5 to 12 months post-discharge

Control: usual care involved follow-up with GP; GPs were sent discharge summaries; "the quality of follow-up care by general practitioners varies in Sweden from non follow-up at all to regular visits every third or fourth month"

Usual care before discharge (I and C): initiation of secondary prevention medications and referral to continuous physiotherapy or occupation therapy, if necessary

Outcomes SBP (12 months); DBP (12 months); recurrent stroke (3.5 years)

General Information

Funding: this study was supported by grants from the Research Fund at Skaraborg Hospital, the Skaraborg Institute for Research and Development, and the Swedish Stroke Association

Country of origin: Sweden

Publication language: English

Notes Analysis method: not stated

Risk of bias: low

\section{Risk of bias}

\section{Bias}

Authors' judgement Support for judgement

Random sequence genera- Low risk Shuffling sealed envelopes tion (selection bias) 
Welin 2010 (Continued)

\begin{tabular}{lll}
$\begin{array}{l}\text { Allocation concealment } \\
\text { (selection bias) }\end{array}$ & Low risk & Shuffling sealed envelopes \\
\hline $\begin{array}{l}\text { Incomplete outcome data } \\
\text { (attrition bias) }\end{array}$ & Low risk & Missing data reported by group \\
All outcomes & $\begin{array}{l}\text { Attrition: I: } 18 / 81 \text { ( } 5 \text { died, } 13 \text { did not attend follow-up visit); C: } 30 / 82 \text { (9 died, } 21 \\
\text { did not attend follow-up visit) }\end{array}$ \\
& $\begin{array}{l}\text { Judgement: reasons for missing data reported and review authors judge that } \\
\text { they are unlikely to be related to study outcomes }\end{array}$
\end{tabular}

Selective reporting (re- Low risk Study protocol available and outcomes are reported in the pre-specified way
porting bias)

\begin{tabular}{ll}
\hline Other bias $\quad$ Low risk & The study appears to be free from other sources of bias \\
\hline
\end{tabular}

AF: atrial fibrillation

AMT: Abbreviated Mental Test

APN: advanced practice nurse

BMI: body mass index

BP: blood pressure

C: control

DBP: diastolic blood pressure

GP: general practitioner

HDL: high density lipoprotein

I: intervention

IQR: interquartile range

LDL: low density lipoprotein

NIHSS: National Institutes of Stroke Scale

RCT: randomised controlled trial

SBP: systolic blood pressure

SD: standard deviation

SE: standard error

TIA: transient ischaemic attack

Characteristics of excluded studies [ordered by study ID]

\begin{tabular}{ll}
\hline Study & Reason for exclusion \\
\hline Amariles 2012 & Outcomes not reported separately for stroke/TIA participants \\
\hline Banet 1997 & No relevant outcomes \\
\hline Bokemark 1996 & No relevant outcomes \\
\hline FIMDM_CVD 2010 & Not a stroke service intervention \\
\hline Gillham 2010 & No relevant outcomes \\
\hline Goessens 2006 & Outcomes not reported separately for stroke/TIA participants \\
\hline Green 2007 & No relevant outcomes \\
\hline Harrington 2007 & Not intended to improve modifiable risk factor control \\
\hline Johnston 2000 & Not a stroke service intervention \\
\hline
\end{tabular}




\begin{tabular}{|c|c|}
\hline Study & Reason for exclusion \\
\hline Joshi 2012 & Outcomes not reported separately for stroke/TIA participants \\
\hline Ma 2009 & Outcomes not reported separately for stroke/TIA participants \\
\hline Middleton 2004 & No relevant outcomes \\
\hline Nir 2006 & No relevant outcomes \\
\hline Ornstein 2004 & Not a stroke service intervention \\
\hline Palanco 2011 & Outcomes not reported separately for stroke/TIA participants \\
\hline Rimmer 2000 & Contained exercise training program \\
\hline Ross 2007 & Not intended to improve modifiable risk factor control \\
\hline Sides 2012 & Not RCT \\
\hline Spassova 2016 & Outcomes not reported separately for stroke participants \\
\hline Strandberg 2006 & Outcomes not reported separately for stroke/TIA participants \\
\hline UMIN000001865 & Contained exercise training program \\
\hline Vernooij 2012 & Outcomes not reported separately for stroke/TIA participants \\
\hline
\end{tabular}

TIA: transient ischaemic attack

Characteristics of studies awaiting classification [ordered by study ID]

ACTRN12608000166370

\begin{tabular}{ll}
\hline Methods & Parallel RCT \\
\hline Participants & Ischaemic/haemorrhagic stroke or TIA \\
\hline Interventions & Co-ordinated team approach for risk factor management in primary care setting \\
\hline Outcomes & $\begin{array}{l}12 \text { months and } 24 \text { months: Framingham cardiovascular disease risk score; use of secondary pre- } \\
\text { vention medications; BP }\end{array}$ \\
\hline Notes & Status: Results awaited (correspondence August 2016) \\
\hline
\end{tabular}

Feld-Glazman 2012

\begin{tabular}{ll}
\hline Methods & Parallel RCT \\
\hline Participants & Stroke \\
\hline Interventions & $\begin{array}{l}\text { Stroke education program; motivational interviewing to facilitate behaviour change for secondary } \\
\text { stroke prevention }\end{array}$ \\
\hline
\end{tabular}


Feld-Glazman 2012 (Continued)
Outcomes
12 weeks: risk factor behaviour

Notes

Status: completed

No study reports available (no correspondence established September 2016)

\section{ISRCTN63816609}

\begin{tabular}{ll}
\hline Methods & Parallel RCT \\
\hline Participants & Acute TIA or ischaemic stroke \\
\hline Interventions & $\begin{array}{l}\text { Nurse-led care pathway of roup clinics addressing smoking cessation, healthy eating, physical ac- } \\
\text { tivity, and the risk factors of stroke }\end{array}$ \\
\hline Outcomes & 6 months: ambulatory 12-hour systolic blood pressure, change in BMI and abdominal obesity \\
\hline Notes & Status: completed \\
& No study reports available (no correspondence established April 2017)
\end{tabular}

\section{ISRCTN95662526}

\begin{tabular}{ll}
\hline Methods & Parallel RCT \\
\hline Participants & Mild stroke \\
\hline Interventions & $\begin{array}{l}\text { Telephone support addressing secondary prevention and adaption; use of written information and } \\
\text { "StrokEngine" website }\end{array}$ \\
\hline Outcomes & 12 months: use of health services and reasons (e.g. recurrent stroke) \\
\hline Notes & Status: completed (June 2012) \\
& No study reports available (no correspondence established September 2016)
\end{tabular}

NCT00211731

\begin{tabular}{ll}
\hline Methods & RCT \\
\hline Participants & Stroke or TIA \\
\hline Interventions & Chronic disease self-management course \\
\hline Outcomes & Adherence to secondary prevention measures \\
\hline Notes & Status: completed \\
& No study reports available (no correspondence established September 2016)
\end{tabular}


NCT00703274

\begin{tabular}{ll}
\hline Methods & RCT \\
\hline Participants & Ischaemic stroke or TIA \\
\hline Interventions & Lay persons ('stroke navigators') trained to help participants reduce their risk of secondary stroke \\
\hline Outcomes & 12 months: LDL; SBP; HbAlc; pill count (antiplatelet medication) \\
\hline Notes & Status: completed; analysing data \\
& No study reports available (no correspondence established September 2016) \\
\hline
\end{tabular}

\section{NCT01071408}

\begin{tabular}{ll}
\hline Methods & RCT \\
\hline Participants & Stroke, TIA \\
\hline Interventions & $\begin{array}{l}\text { Outpatient stroke prevention program involving group clinics, patient self-management and tele- } \\
\text { phone care co-ordination }\end{array}$ \\
\hline Outcomes & 3 months and 7 months: BP; lipids; medication adherence \\
\hline Notes & Status: completed (31 May 2012); analysing data \\
& No study reports available (no correspondence established September 2016)
\end{tabular}

NCT01122394

\begin{tabular}{ll}
\hline Methods & Parallel RCT \\
\hline Participants & Stroke or TIA \\
\hline Interventions & Telephone intervention to reduce behavioural risk factors for secondary stroke \\
\hline Outcomes & 6 months: BP; total cholesterol/HDL ratio; antihypertensive/lipid-lowering medication adherence \\
\hline Notes & Status: results awaited (no correspondence established September 2016) \\
\hline
\end{tabular}

\section{NCT01807793}

\begin{tabular}{ll}
\hline Methods & Parallel RCT \\
\hline Participants & Stroke or TIA \\
\hline Interventions & Psycho-education (individual and group sessions) \\
\hline Outcomes & $\begin{array}{l}\text { 3 months and } 6 \text { months: adherence to secondary prevention medications, blood pressure, HbA1c, } \\
\text { BMI, cholesterol, triglycerides }\end{array}$ \\
\hline
\end{tabular}


NCT01807793 (Continued)
Notes
Status: completed - no contact established 2016

\section{NCT02140658}

\begin{tabular}{ll}
\hline Methods & Parallel assignment \\
\hline Participants & Ischaemic stroke \\
\hline Interventions & Multiple health education interventions \\
\hline Outcomes & Medication adherence at 3,6 and 12 months \\
\hline Notes & Status: completed - results awaited \\
\hline
\end{tabular}

\section{Redfern 2007}

\begin{tabular}{ll}
\hline Methods & Cluster RCT \\
\hline Participants & Stroke \\
\hline Interventions & $\begin{array}{l}\text { Individualised evidence-based secondary prevention plans provided to participants/caregivers } \\
\text { ("keeping well plans") and GPs ("secondary prevention plans") on a maximum of } 3 \text { occasions (10 } \\
\text { weeks, } 5 \text { months and } 8 \text { months post-stroke); structured approach to risk factor monitoring }\end{array}$
\end{tabular}

\section{Outcomes}

12 months: modifiable risk factors for stroke: blood pressure, total cholesterol, $\mathrm{HbAlc}, \mathrm{BMI}$

Outcome data relevant to the review not available (no correspondence established September 2016)

BMI: body mass index

BP: blood pressure

DBP: diastolic blood pressure

GP: general practitioner

LDL: low density lipoprotein

RCT: randomised controlled trial

SBP: systolic blood pressure

TIA: transient ischaemic attack

Characteristics of ongoing studies [ordered by study ID]

\section{ACTRN12615000888561}

\begin{tabular}{ll}
\hline Study name & A conversation with patients about medications after a stroke \\
\hline Methods & RCT \\
\hline Participants & Stroke/TIA \\
\hline Interventions & Patient-centred educational exchange \\
\hline
\end{tabular}


ACTRN12615000888561 (Continued)

Outcomes $\quad 0,3$ and 12 months - self reported medication adherence, BP and cholesterol

\begin{tabular}{ll}
\hline Starting date & Start: December 2015 \\
& Estimated completion: October 2017 \\
\hline Contact information & $\begin{array}{l}\text { Judith Coombes, Pharmacy Department Princess Alexandra Hospital 199 Ipswich Rd Woolloongab- } \\
\text { ba QLD, Australia } \\
\text { Contact: judith.coombes@health.qld.gov.au }\end{array}$ \\
\hline Notes & Status: recruiting \\
\hline
\end{tabular}

\section{ChicTR-TQR-14004950}

\begin{tabular}{ll}
\hline Study name & $\begin{array}{l}\text { Construction of "hospital-community-family" transitional care model for elderly hypertensive pa- } \\
\text { tients based on information platform }\end{array}$ \\
\hline Methods & Quasi-randomised controlled \\
\hline Participants & Stroke \\
\hline Interventions & Nurse follow up \\
\hline Outcomes & Start: December 2014 \\
\hline Starting date & Yuying Shi, 19 Qi Xiu Road, Nantong, Jiangsu Province China \\
\hline Contact information & Contact: 675224943@qq.com \\
\hline Notes & Status: contact not achieved \\
\hline
\end{tabular}

\section{ChiCTR-TRC-12002127}

\begin{tabular}{ll}
\hline Study name & $\begin{array}{l}\text { Effects of clinical pharmacist interventions on the secondary prevention in the ischaemic stroke pa- } \\
\text { tients }\end{array}$ \\
\hline Methods & Parallel RCT \\
\hline Participants & Ischaemic stroke \\
\hline Interventions & Pharmacist-led individualised pharmaceutical care \\
\hline Outcomes & $\begin{array}{l}\text { Stroke recurrence, myocardial infarction, vascular death, medication compliance, body weight, } \\
\text { blood pressure, serum glucose, serum lipids }\end{array}$ \\
\hline Starting date & Start: April 2012 \\
\hline Contact information & Estimated completion: unknown
\end{tabular}


ChicTR-TRC-12002127 (Continued)

Contact: haibindai@163.com

Notes Status: ongoing study (correspondence August 2016)

\section{COACH 2014}

\begin{tabular}{ll}
\hline Study name & Healthy lifestyles after stroke (Stroke Coach) \\
\hline Methods & Parallel RCT \\
\hline Participants & Experienced a stroke in the last 12 months, > 50 years \\
\hline Interventions & Telephone administered lifestyle coaching sessions \\
\hline Outcomes & 0,6 and 12 months - medication adherence, BP, lipid and glucose profile, BMI \\
\hline Starting date & Start: July 2014 \\
& Estimated completion: January 2017 \\
\hline Contact information & Chihya Hung, University Hospital of Northern BC, Prince George, BC, Canada \\
\hline Notes & Contact: Chihya.Hung@ubc.ca \\
\hline
\end{tabular}

\section{DESERVE 2014}

\begin{tabular}{ll}
\hline Study name & Discharge Educational Strategies for Reduction of Vascular Events (DESERVE) \\
\hline Methods & Parallel RCT \\
\hline Participants & $\begin{array}{l}\text { Mild ischaemic cerebral infarction/intracerebral haemorrhage/TIA, > 18 years age; vascular risk fac- } \\
\text { tors }\end{array}$ \\
\hline Interventions & $\begin{array}{l}\text { Education on stroke preparedness plus risk factor reduction education, and help accessing follow } \\
\text { up care with health workers }\end{array}$ \\
\hline Outcomes & $\begin{array}{l}6 \text { and } 12 \text { months: BP, secondary incident } \\
\text { Starting date }\end{array}$ \\
Start: April 2013 \\
Estimated completion: March 2017 \\
\hline Bernadette Boden-Albala, NYU Langone Medical Center, New York, NY, USA, 10016 \\
Contact: 212-659-9322 \\
\hline States
\end{tabular}


DMP 2014

\begin{tabular}{|c|c|}
\hline Study name & The effects of disease management programs for prevention of recurrent ischemic stroke \\
\hline Methods & Parallel RCT \\
\hline Participants & Ischaemic stroke/TIA \\
\hline Interventions & Disease management program include self management education provided by a nurse \\
\hline Outcomes & 2.5 years: Framingham Risk Score; weight; BMI; BP; cholesterol; HbA1c \\
\hline \multirow[t]{2}{*}{ Starting date } & Start: January 2014 \\
\hline & Estimated completion: January 2017 \\
\hline Notes & Status: active, not recruiting \\
\hline
\end{tabular}

\section{Feldman 2015}

\begin{tabular}{ll}
\hline Study name & Center for Stroke Disparities Solution (CSDS) - community transitions intervention \\
\hline Methods & Parallel RCT \\
\hline Participants & Stroke or TIA \\
\hline Interventions & $\begin{array}{l}\text { Either usual care, nurse practitioner and health coach or nurse practitioner only. Self-management } \\
\text { coaching }\end{array}$ \\
\hline Outcomes & \begin{tabular}{l} 
3 and 6 months systolic BP, weight loss and medication adherence \\
\hline Starting date
\end{tabular} \\
\hline Start: September 2012 \\
\hline Estimated completion: August 2018 \\
\hline $\begin{array}{l}\text { Margaret M McDonald, Visiting Nurse Service of New York, National Institute of Neurological Disor- } \\
\text { ders and Stroke, New York University School of Medicine, NY, USA }\end{array}$ \\
\hline Contact: Margaret.McDonald@VNSNY.org \\
\hline Status: recruiting
\end{tabular}

\section{ISRCTN07607027}

\begin{tabular}{ll}
\hline Study name & Promoting Adherence to a Regimen of risk factor modification by Trained Non-medical personnel \\
Evaluated against Regular practice Study PARTNERS
\end{tabular}

\begin{tabular}{ll}
\hline Methods & RCT \\
\hline Participants & TIA or non-disabling stroke; hypertension \\
\hline Interventions & Support from a trained volunteer for risk factor reduction \\
\hline
\end{tabular}




\section{ISRCTN07607027 (Continued)}

Outcomes

12 months and 24 months: DBP; medication adherence; BMI; cardiovascular risk score; LDL; total cholesterol/HDL ratio; HbA1c

Starting date

Start: April 2009

Estimated completion: 30 September 2017

Contact information Richard Chan

Contact: 339 Windermere Rd, Rm B10-118, University Hospital, N6A 5A5, London, Canada

Notes

Status: ongoing/recruiting (correspondence August 2016)

\section{ISRCTN08913646}

\begin{tabular}{ll}
\hline Study name & $\begin{array}{l}\text { The effect of a Health Empowerment Intervention for Stroke Self-management (HEISS) on the self- } \\
\text { management behaviour and health outcomes of stroke rehabilitation patients }\end{array}$ \\
\hline Methods & Parallel RCT \\
\hline Participants & Stroke \\
\hline Interventions & $\begin{array}{l}\text { Stroke self-management intervention (involves group education and nurse-led telephone fol- } \\
\text { low-up) }\end{array}$ \\
\hline Outcomes & \begin{tabular}{l} 
Stroke recurrence, self-management behaviour \\
\hline Starting date
\end{tabular} \\
$\begin{array}{l}\text { Start: May 2012 } \\
\text { Estimated completion: May 2014 }\end{array}$ \\
\hline Contact information & Kong, Janet Sit, The Nethersole School of Nursing, Faculty of Medicine, Chinese University of Hong \\
\hline Notes & Status: ongoing (correspondence April 2013 - no correspondence established 2016) \\
\hline
\end{tabular}

\section{ISRCTN97412358}

\begin{tabular}{ll}
\hline Study name & ECG monitoring to detect atrial fibrillation after stroke \\
\hline Methods & RCT \\
\hline Participants & Ischaemic stroke or TIA \\
\hline Interventions & Continuous ECG monitoring to detect atrial fibrillation after acute stroke or TIA \\
\hline Outcomes & 12 months: recurrent stroke \\
\hline Starting date & Start: May 2010 \\
& Estimated completion: December 2016 \\
\hline Contact information & $\begin{array}{l}\text { Professor Kennedy R Lees, Acute Stroke Unit \& Cerebrovascular Clinic, Western Infirmary, Glasgow, } \\
\text { UK }\end{array}$
\end{tabular}


ISRCTN97412358 (Continued)

Contact: k.r.lees@clinmed.gla.ac.uk

Notes Status: ongoing

\section{NCT01517542}

\begin{tabular}{|c|c|}
\hline Study name & Evaluation of effectiveness of nutritional counselling in patients after stroke \\
\hline Methods & Parallel RCT \\
\hline Participants & Stroke \\
\hline Interventions & $\begin{array}{l}\text { Nutritional counselling (participants received written guidance to promote adherence to 'DASH' di- } \\
\text { et recommendations) }\end{array}$ \\
\hline Outcomes & 30 days; 3, 6, 9 and 12 months: body weight, blood glucose, blood pressure, lipid profile \\
\hline \multirow[t]{2}{*}{ Starting date } & Strart: February 2010 \\
\hline & Estimated completion: February 2012 \\
\hline \multirow[t]{2}{*}{ Contact information } & $\begin{array}{l}\text { Sheila CO Martins, PI; Vanessa A Piper, SI, Hospital de Clínicas de Porto Alegre, Porto Alegre, RS, } \\
\text { Brazil, } 90035903\end{array}$ \\
\hline & $\begin{array}{l}\text { Contact: mailto:smartins\%40portoweb.com?subject=NCT01517542, 10-0014, Evaluation of Effec- } \\
\text { tiveness of Nutritional Counseling in Patients After Stroke; mailto:vanalves001\%40gmail.com?sub- } \\
\text { ject=NCT01517542, 10-0014, Evaluation of Effectiveness of Nutritional Counseling in Patients After } \\
\text { Stroke }\end{array}$ \\
\hline Notes & Status: recruiting participants (correspondence April 2013 - no correspondence established 2016) \\
\hline
\end{tabular}

\section{NCT01586702}

\section{Study name}

Intensified Secondary Prevention Intending a Reduction of Recurrent Events in TIA and Minor Stroke Patients (INSPiRE-TMS). A randomized trial comparing a patient centred support program versus conventional care

\begin{tabular}{ll}
\hline Methods & Parallel RCT \\
\hline Participants & TIA or minor stroke \\
\hline Interventions & $\begin{array}{l}\text { "Stepwise intensified patient support program" delivered in outpatient clinics over } 2 \text { years (par- } \\
\text { ticipants are provided with individualised risk factor data and supported in finding physical activi- } \\
\text { ties/smoking cessation programs) }\end{array}$
\end{tabular}

Outcomes

3.5 years and 6 years: major vascular events (including stroke, TIA and major coronary events)
Start: September 2011

Estimated completion: June 2017

Contact information

Heinrich J Audebert, MD, Department of Neurology, Charité Universitätsmedizin Berlin, Germany, 12200 
NCT01586702 (Continued)

Contact: mailto:heinrich.audebert\%40charite.de?subject=NCT01586702, EA2/084/11, Intensified Secondary Prevention Intending a Reduction of Recurrent Events in TIA and Minor Stroke Patients

Notes Status: recruiting participants

\section{NCT01776034}

\begin{tabular}{ll}
\hline Study name & Health promotion and wellness program for stroke survivors \\
\hline Methods & Parallel RCT \\
\hline Participants & Stroke \\
\hline Interventions & $\begin{array}{l}\text { Health promotion program to reduce body weight (involving lifestyle counselling delivered through } \\
\text { group education and telephone follow-up) }\end{array}$ \\
\hline Outcomes & \begin{tabular}{l} 
3 months and 6 months: body weight, biomarkers (cholesterol, triglycerides, HbA1c) \\
\hline Starting date
\end{tabular} \\
$\begin{array}{l}\text { Start: January 2013 } \\
\text { Estimated completion: July 2015 }\end{array}$ \\
\hline Contact information & $\begin{array}{l}\text { Contact: mailto:mcdanic3\%40ccf.org?subject=NCT01776034, 11-847, Health Promotion and Well- } \\
\text { ness Program for Stroke Survivors }\end{array}$ \\
\hline Notes & Status: recruiting participants \\
\hline
\end{tabular}

\section{NCT01812421}

\begin{tabular}{ll}
\hline Study name & $\begin{array}{l}\text { A nested case-control study on the secondary prevention of ischemic stroke and TIA by Hyperten- } \\
\text { sion Health Education Protocol (HHEP): the Post-Stroke Preventive Trial }\end{array}$ \\
\hline Methods & Parallel Assignment \\
\hline Participants & Ischaemic stroke or TIA \\
\hline Interventions & Health education tailored for hypertension \\
\hline Outcomes & Stroke recurrence at 1 year \\
\hline Starting date & Start: April 2013 \\
\hline Estimated completion: April 2015 \\
\hline Dontact information & Guangzhou, Guangdong, China, 510120 \\
& Contact: zizi_33@126.com \\
\hline Notes & Status: recruiting participants \\
\hline
\end{tabular}


NCT02132364

\begin{tabular}{ll}
\hline Study name & Controlled Education Of Patients after Stroke (CEOPS) \\
\hline Methods & Parallel assignment \\
\hline Participants & First stroke, transient or permanent, ischaemic or haemorrhagic \\
\hline Interventions & $\begin{array}{l}\text { Nurse follow-up, including therapeutic follow-up and an educational program directed to the par- } \\
\text { ticipants and carers }\end{array}$ \\
\hline Outcomes & BP at 1 year \\
\hline Starting date & Start: January 2014 \\
\hline Estimated completion: July 2017 \\
\hline Dontact information & Contact: +33 (0)3 20 44 54 49, regis.bordet@univ-lille2.fr \\
\hline Notes & Status: recruiting participants \\
\hline
\end{tabular}

\section{NCT02140619}

Study name $\quad$ Multiple health education interventions for medication compliance and clinical prognosis of is-
chemic stroke patients

\begin{tabular}{ll}
\hline Methods & Parallel assignment \\
\hline Participants & Acute ischaemic stroke \\
\hline Interventions & $\begin{array}{l}\text { Health education manuals and Digital Video Disc (DVD) during hospitalisation and regular text mes- } \\
\text { sage during } 1 \text { year after discharge }\end{array}$ \\
\hline Outcomes & 3, 6 and 12 month medication adherence \\
\hline Starting date & Start: May 2014 \\
& Estimated completion: September 2015 \\
\hline Contact information & Dr Zixiao Li, Beijing Tian Tan Hospital, Capital Medical University, Beijing, China, 100050 \\
\hline Cotes & Status: recruiting participants \\
\hline
\end{tabular}

\section{NCT02156778}

\begin{tabular}{ll}
\hline Study name & Post-stroke disease management - Stroke Card (Stroke Card) \\
\hline Methods & Parallel assignment \\
\hline Participants & Ischemic stroke \\
\hline
\end{tabular}




\section{NCT02156778 (Continued)}

Interventions

\section{Outcomes}

Starting date$$
\text { Starting date }
$$

Estimated completion: March 2017

Contact information

Dr Stefan Kiechl, Department of Neurology, Medical University Innsbruck, Innsbruck, Tyrol, Austria, 6020

Contact: +43-512-504- ext 24244; stefan.kiechl@i-med.ac.at

Notes Status: recruiting participants

\section{NCT02251834}

\begin{tabular}{ll}
\hline Study name & Hispanic Secondary Stroke Prevention Initiative (HISSPI) \\
\hline Methods & Parallel assignment \\
\hline Participants & History of an ischaemic or intracerebral haemorrhagic stroke within the past 5 years \\
\hline Interventions & $\begin{array}{l}\text { Community health worker to deliver care at home, via telephone or mobile technology or group } \\
\text { work to minimise risk factors in post stroke patients }\end{array}$ \\
\hline Outcomes & 12 months BP, LDL, self-reported adherence to statins and anti-platelets and HbA1C \\
\hline Starting date & Start: January 2015 \\
\hline Contact information & Dr Olveen Carrasquillo University of Miami, Miami, FL, USA \\
\hline Notes & Contact: $305-243-5505$ \\
\hline
\end{tabular}

\section{NCT02712385}

\begin{tabular}{ll}
\hline Study name & SPRITE - a feasibility and pilot study \\
\hline Methods & Parallel assignment \\
\hline Participants & TIA \\
\hline Interventions & Novel home-based programme manual \\
\hline Outcomes & Level of physical activity, BMI, BP at 12 weeks \\
\hline Starting date & Start: March 2016 \\
& Estimated completion: February 2018 \\
\hline
\end{tabular}


NCT02712385 (Continued)

Contact information

Dr Neil Heron, Ulster Hospital, Belfast, Antrim, United Kingdom

Contact: 0289097 ext 6064,nheron02@qub.ac.uk

Notes Status: recruiting participants

\section{NCT02868723}

Study name

PROspective Study to OPTimize thE HEALTH of Patients With TIAs (Transient Ischemic Attacks) and Stroke Admitted to the Hamad General Hospital (PROMOTE-HEALTH)

\begin{tabular}{ll}
\hline Methods & Parallel assignment \\
\hline Participants & Ischaemic stroke \\
\hline Interventions & Nurse and pharmacist follow-up \\
\hline Outcomes & BP and LDL at 1 year
\end{tabular}

\section{Start: October 2016}

Estimated completion: December 2018

Contact information Dr Yahia Bashier, Hamad Medical Corporation, Qatar

Contact: 55246887, yimam@hamad.qa

Notes Status: not yet recruiting participants

\section{Sarfo 2016}

\begin{tabular}{ll}
\hline Study name & Phone-based Intervention under Nurse Guidance after Stroke (PINGS) \\
\hline Methods & Parallel RCT \\
\hline Participants & Stroke \\
\hline Interventions & Nurse-directed mobile health technology to promote adherence to antihypertensive medication \\
\hline Outcomes & 9-month BP and medication adherence \\
\hline Starting date & Start: November 2016 \\
& Estimated completion: June 2017 \\
\hline Contact information & $\begin{array}{l}\text { Stephen Sarfo, Division of Neurology, Department of Medicine, Kwame Nkrumah University of } \\
\text { Science and Technology, Kumasi, Ghana } \\
\text { Contact: Stephensarfo78@gmail.com }\end{array}$ \\
\hline Notes & Status: not yet recruiting \\
\hline
\end{tabular}


Spruill 2015

\begin{tabular}{ll}
\hline Study name & Practice-based trial of home BP telemonitoring among minority stroke survivors \\
\hline Methods & Parallel RCT \\
\hline Participants & Ischaemic or haemorrhagic stroke \\
\hline Interventions & Home BP telemonitoring protocol with counselling telephone calls with a nurse case manager \\
\hline Outcomes & $\begin{array}{l}\text { 12 month BP, 24 month stroke recurrence, 6, } 12 \text { and 24 months lipid, blood glucose, weight loss } \\
\text { and medication adherence }\end{array}$ \\
\hline Starting date & Start: Decemeber 2013 \\
\hline Estimated completion: December 2018 \\
\hline Gontact information & Contact: olugbenga.ogedegbe@nyumc.org \\
\hline Notes & Status: Rrecruiting \\
\hline
\end{tabular}

\section{THRIVES 2013}

\begin{tabular}{ll}
\hline Study name & Tailored Hospital-based Risk reduction to Impede Vascular Events after Stroke (THRIVES) \\
\hline Methods & Parallel RCT \\
\hline Participants & Stroke \\
\hline Interventions & Pre-appointment phone text, In-clinic educational video, patient report card, post-clinic phone text \\
\hline Outcomes & 12 month BP and vascular event \\
\hline Starting date & Start: September 2014 \\
\hline Contact information & Estimated completion: June 2017 \\
\hline Notes & Rufus Akinyemi, Sacred Heart Hospital, Medical School of Carolina Country \\
\hline
\end{tabular}

\section{Towfighi 2013}

\begin{tabular}{ll}
\hline Study name & $\begin{array}{l}\text { Secondary stroke prevention by Uniting Community and Chronic care model teams Early to End } \\
\text { Disparities: the SUCCEED Trial }\end{array}$ \\
\hline Methods & Parallel RCT \\
\hline Participants & Stroke \\
\hline
\end{tabular}


Towfighi 2013 (Continued)

Interventions
Care manager (nurse practitioner or physician assistant) to implement protocols for secondary prevention; group education sessions on chronic disease self-management; home visits from a community health worker; participants provided with blood pressure monitors

Outcomes 12 months: SBP, dyslipidaemia, HbA1c, BMI, vascular events, medication adherence

\begin{tabular}{ll}
\hline Starting date & Start: September 2013 \\
& Estimated completion: August 2017 \\
\hline Contact information & $\begin{array}{l}\text { Barbara G Vickrey, MD, MPH; Amytis Towfighi, MD, Rancho Los Amigos National Rehabilitation Cen- } \\
\text { ter, Downey, CA, USA, } 90242\end{array}$ \\
\hline Notes & Status: enrolling participants by invitation only \\
\hline
\end{tabular}

BMI: body mass index

BP: blood pressure

CVD: cardiovascular disease

DBP: diastolic blood pressure

ECG: electrocardiogram

GP: general practitioner

LDL: low density lipoprotein

$\mathrm{RCT}$ : randomised controlled trial

SBP: systolic blood pressure

TIA: transient ischaemic attack

\section{DATA AND ANALYSES}

\section{Comparison 1. Educational or behavioural interventions for patients versus usual care}

\begin{tabular}{|c|c|c|c|c|}
\hline Outcome or subgroup title & No. of studies & $\begin{array}{l}\text { No. of partici- } \\
\text { pants }\end{array}$ & Statistical method & Effect size \\
\hline $\begin{array}{l}1.1 \text { Mean systolic blood pres- } \\
\text { sure }\end{array}$ & 11 & 1398 & $\begin{array}{l}\text { Mean Difference (IV, Random, 95\% } \\
\mathrm{CI} \text { ) }\end{array}$ & $-2.81[-7.02,1.39]$ \\
\hline $\begin{array}{l}1.2 \text { Mean diastolic blood pres- } \\
\text { sure }\end{array}$ & 11 & 1398 & $\begin{array}{l}\text { Mean Difference (IV, Random, 95\% } \\
\mathrm{CI} \text { ) }\end{array}$ & $-0.83[-2.80,1.13]$ \\
\hline $\begin{array}{l}1.3 \text { Blood pressure target } \\
\text { achievement }\end{array}$ & 3 & 266 & Odds Ratio (M-H, Random, 95\% Cl) & $0.74[0.39,1.44]$ \\
\hline 1.4 Mean total cholesterol & 7 & 721 & $\begin{array}{l}\text { Mean Difference (IV, Random, 95\% } \\
\mathrm{CI})\end{array}$ & $0.10[-0.28,0.47]$ \\
\hline $\begin{array}{l}1.5 \text { Total cholesterol target } \\
\text { achievement }\end{array}$ & 1 & 56 & Odds Ratio (M-H, Random, 95\% Cl) & $1.78[0.60,5.30]$ \\
\hline $\begin{array}{l}\text { 1.6 Mean low density lipopro- } \\
\text { tein }\end{array}$ & 4 & 495 & $\begin{array}{l}\text { Mean Difference (IV, Random, 95\% } \\
\mathrm{CI})\end{array}$ & $-0.13[-0.28,0.02]$ \\
\hline $\begin{array}{l}\text { 1.7 Mean high density lipopro- } \\
\text { tein }\end{array}$ & 3 & 452 & $\begin{array}{l}\text { Mean Difference (IV, Random, 95\% } \\
\mathrm{Cl} \text { ) }\end{array}$ & $-0.03[-0.11,0.05]$ \\
\hline
\end{tabular}




\begin{tabular}{|c|c|c|c|c|}
\hline Outcome or subgroup title & No. of studies & $\begin{array}{l}\text { No. of partici- } \\
\text { pants }\end{array}$ & Statistical method & Effect size \\
\hline 1.8 Mean triglycerides & 3 & 182 & $\begin{array}{l}\text { Mean Difference (IV, Random, 95\% } \\
\text { CI) }\end{array}$ & $-0.01[-0.31,0.30]$ \\
\hline 1.9 Mean HbA1c & 1 & 70 & $\begin{array}{l}\text { Mean Difference (IV, Random, 95\% } \\
\mathrm{CI})\end{array}$ & $-0.11[-0.39,0.17]$ \\
\hline $\begin{array}{l}\text { 1.10 HbA1C target achieve- } \\
\text { ment }\end{array}$ & 1 & 67 & Odds Ratio (M-H, Random, 95\% Cl) & $1.53[0.57,4.08]$ \\
\hline 1.11 Mean BMI & 2 & 127 & $\begin{array}{l}\text { Mean Difference (IV, Random, 95\% } \\
\mathrm{CI} \text { ) }\end{array}$ & $0.22[-0.85,1.29]$ \\
\hline $\begin{array}{l}\text { 1.12 Proporation of partici- } \\
\text { pants with secondary stroke }\end{array}$ & 4 & 4333 & Odds Ratio (M-H, Random, 95\% Cl) & $0.82[0.37,1.84]$ \\
\hline 1.13 Number of secondary TIAs & 2 & 4207 & Odds Ratio (M-H, Random, 95\% Cl) & $1.09[0.52,2.30]$ \\
\hline $\begin{array}{l}\text { 1.14 Number of myocardial in- } \\
\text { farctions }\end{array}$ & 3 & 4277 & Odds Ratio (M-H, Random, 95\% Cl) & $0.53[0.17,1.65]$ \\
\hline $\begin{array}{l}1.15 \text { Number of cardiovascular } \\
\text { deaths }\end{array}$ & 1 & 386 & Odds Ratio (M-H, Random, 95\% Cl) & $1.34[0.30,6.07]$ \\
\hline
\end{tabular}

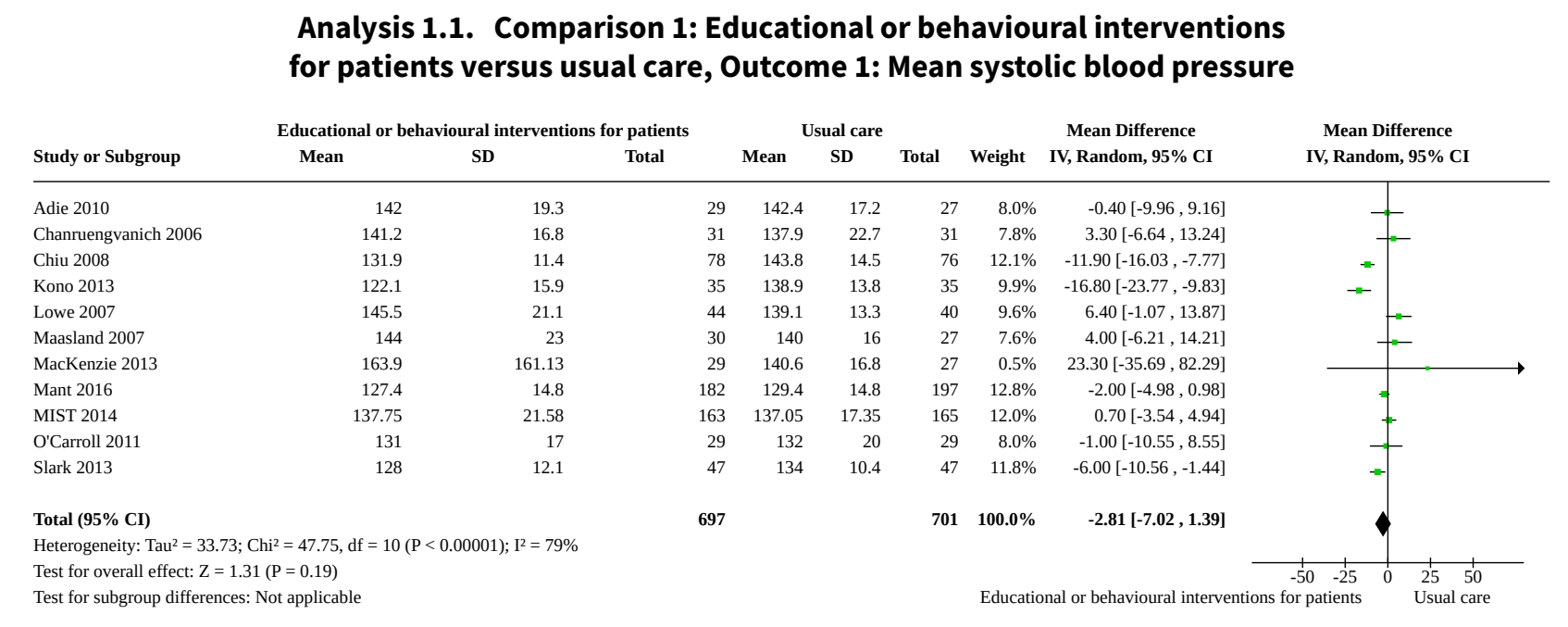


Analysis 1.2. Comparison 1: Educational or behavioural interventions for patients versus usual care, Outcome 2: Mean diastolic blood pressure

\begin{tabular}{|c|c|c|c|c|c|c|c|c|c|c|}
\hline \multirow[b]{2}{*}{ Study or Subgroup } & \multicolumn{3}{|c|}{ Educational or behavioural interventions for patients } & \multicolumn{3}{|c|}{ Usual care } & \multirow[b]{2}{*}{ Weight } & \multirow{2}{*}{$\begin{array}{c}\text { Mean Difference } \\
\text { IV, Random, 95\% CI }\end{array}$} & \multirow{2}{*}{\multicolumn{2}{|c|}{$\begin{array}{c}\text { Mean Difference } \\
\text { IV, Random, 95\% CI }\end{array}$}} \\
\hline & Mean & SD & Total & Mean & SD & Total & & & & \\
\hline Adie 2010 & 75.7 & 10.1 & 29 & 72.1 & 12.1 & 27 & $7.0 \%$ & $3.60[-2.26,9.46]$ & & 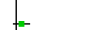 \\
\hline Chanruengvanich 2006 & 77.1 & 11.3 & 31 & 75.8 & 11.5 & 31 & $7.3 \%$ & $1.30[-4.38,6.98]$ & & \\
\hline Chiu 2008 & 76 & 7.8 & 78 & 80.9 & 10 & 76 & $13.4 \%$ & $-4.90[-7.74,-2.06]$ & & $=$ \\
\hline Kono 2013 & 72.9 & 9.5 & 35 & 80.7 & 10.7 & 35 & $8.9 \%$ & $-7.80[-12.54,-3.06]$ & & - \\
\hline Lowe 2007 & 78.5 & 13.3 & 44 & 76.2 & 8.1 & 40 & $9.1 \%$ & $2.30[-2.36,6.96]$ & & \\
\hline Maasland 2007 & 84 & 10 & 30 & 86 & 8 & 27 & $9.1 \%$ & $-2.00[-6.68,2.68]$ & & \\
\hline MacKenzie 2013 & 106.9 & 172 & 29 & 77.8 & 8.9 & 27 & $0.1 \%$ & $29.10[-33.59,91.79]$ & & \\
\hline Mant 2016 & 79.9 & 10 & 182 & 80.4 & 9.8 & 197 & $15.6 \%$ & $-0.50[-2.50,1.50]$ & & \\
\hline MIST 2014 & 77.77 & 12.58 & 163 & 77.46 & 11.66 & 165 & $14.0 \%$ & $0.31[-2.32,2.94]$ & & \\
\hline O'Carroll 2011 & 80 & 11 & 29 & 79 & 13 & 29 & $6.5 \%$ & $1.00[-5.20,7.20]$ & & 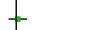 \\
\hline Slark 2013 & 74 & 11 & 47 & 73 & 12.6 & 47 & $8.9 \%$ & $1.00[-3.78,5.78]$ & & - \\
\hline Total $(95 \%$ CI $)$ & & & 697 & & & 701 & $100.0 \%$ & $-0.83[-2.80,1.13]$ & & \\
\hline \multicolumn{11}{|c|}{ Heterogeneity: $\mathrm{Tau}^{2}=5.40 ; \mathrm{Chi}^{2}=23.41, \mathrm{df}=10(\mathrm{P}=0.009) ; \mathrm{I}^{2}=57 \%$} \\
\hline \multirow{2}{*}{\multicolumn{7}{|c|}{$\begin{array}{l}\text { Test for overall effect: } \mathrm{Z}=0.83(\mathrm{P}=0.41) \\
\text { Test for subgroup differences: Not applicable }\end{array}$}} & & -100 & -50 & 50 \\
\hline & & & & & & & Educatio & nal or behavioural interventions & r patients & Usual ca \\
\hline
\end{tabular}

Analysis 1.3. Comparison 1: Educational or behavioural interventions for patients versus usual care, Outcome 3: Blood pressure target achievement

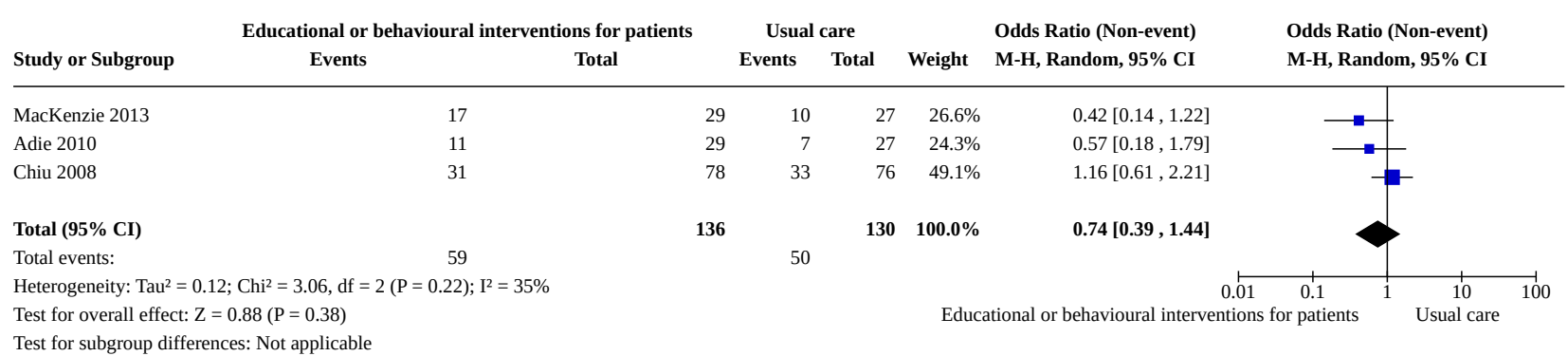

\section{Analysis 1.4. Comparison 1: Educational or behavioural interventions for patients versus usual care, Outcome 4: Mean total cholesterol}

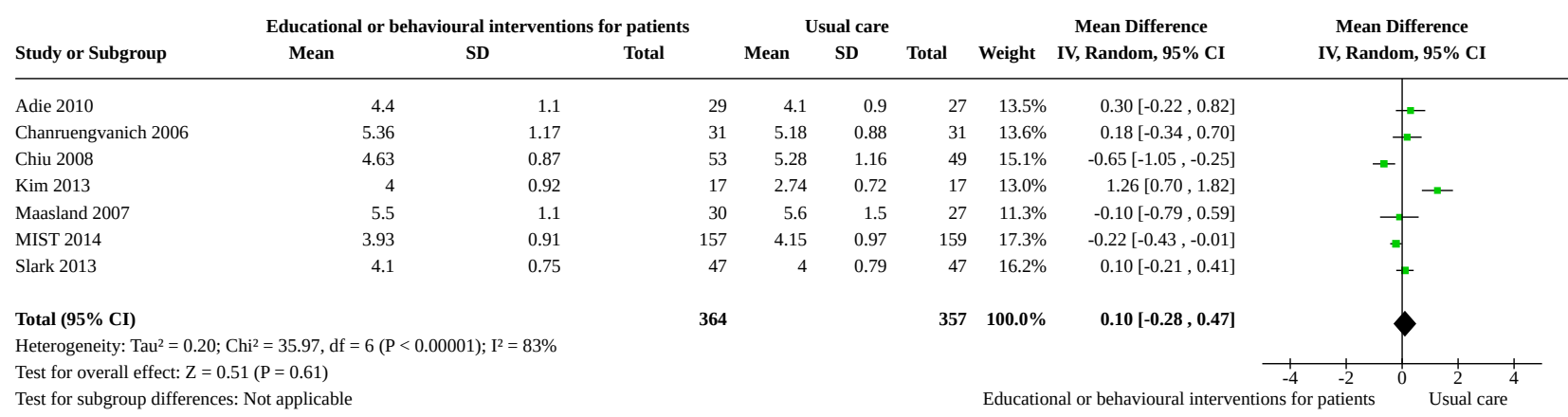


Analysis 1.5. Comparison 1: Educational or behavioural interventions for patients versus usual care, Outcome 5: Total cholesterol target achievement

\begin{tabular}{|c|c|c|c|c|c|c|c|}
\hline \multirow[b]{2}{*}{ Study or Subgroup } & \multicolumn{2}{|c|}{ Usual care } & \multicolumn{2}{|c|}{ Usual care } & \multicolumn{2}{|r|}{ Odds Ratio (Non-event) } & Odds Ratio (Non-event) \\
\hline & Events & Total & Events & Total & Weight & M-H, Random, 95\% CI & M-H, Random, 95\% CI \\
\hline
\end{tabular}

\begin{tabular}{|c|c|c|c|c|c|c|}
\hline Adie 2010 & 9 & 29 & 12 & 27 & $100.0 \%$ & $1.78[0.60,5.30]$ \\
\hline Total $(95 \%$ CI) & & 29 & & 27 & $100.0 \%$ & $1.78[0.60,5.30]$ \\
\hline Total events: & 9 & & 12 & & & \\
\hline
\end{tabular}

Heterogeneity: Not applicable

Test for overall effect: $\mathrm{Z}=1.03(\mathrm{P}=0.30)$

Test for subgroup differences: Not applicable

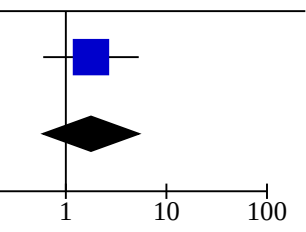

Usual care

Analysis 1.6. Comparison 1: Educational or behavioural interventions for patients versus usual care, Outcome 6: Mean low density lipoprotein

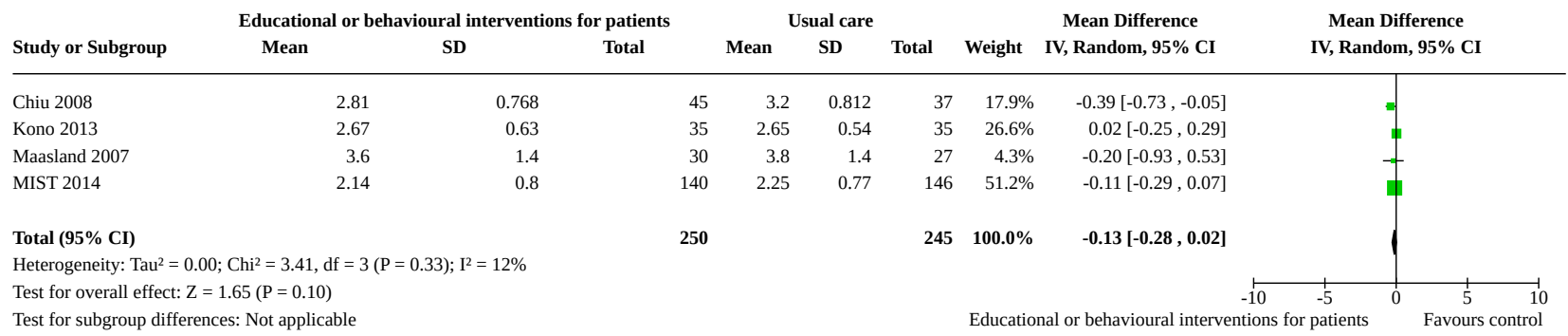

Analysis 1.7. Comparison 1: Educational or behavioural interventions for patients versus usual care, Outcome 7: Mean high density lipoprotein

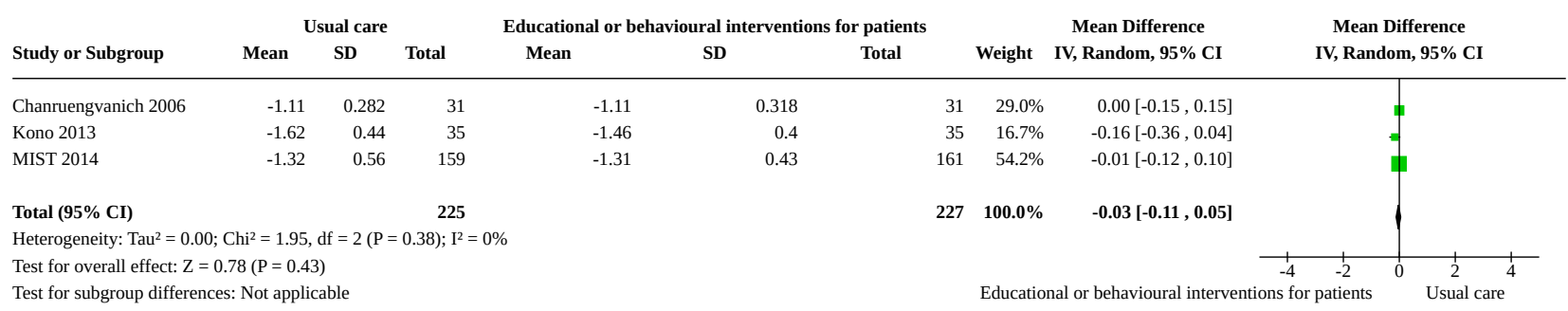

Analysis 1.8. Comparison 1: Educational or behavioural interventions for patients versus usual care, Outcome 8: Mean triglycerides

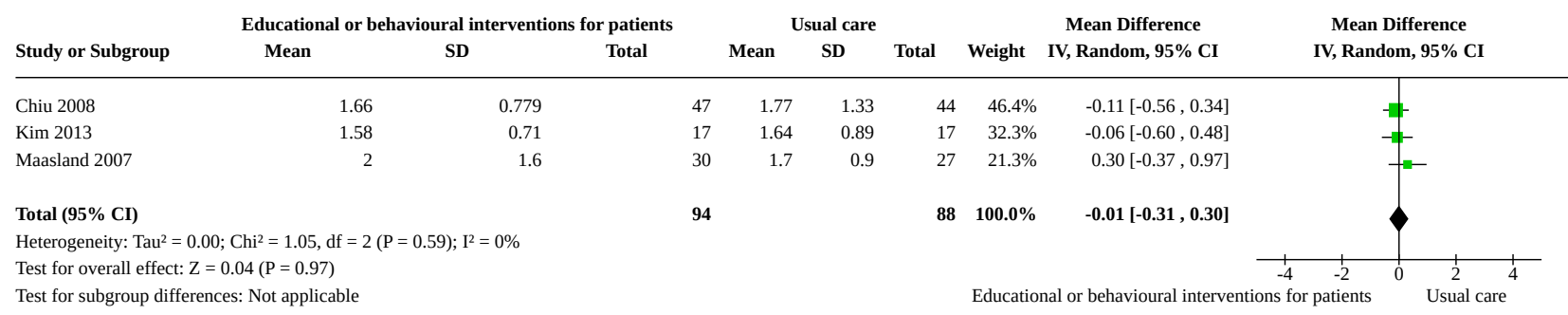


Analysis 1.9. Comparison 1: Educational or behavioural interventions for patients versus usual care, Outcome 9: Mean HbA1c

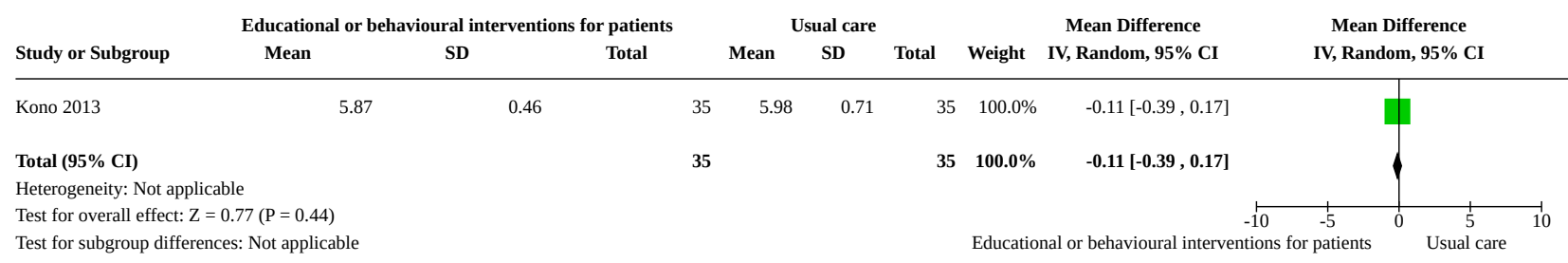

Analysis 1.10. Comparison 1: Educational or behavioural interventions for patients versus usual care, Outcome 10: HbA1C target achievement

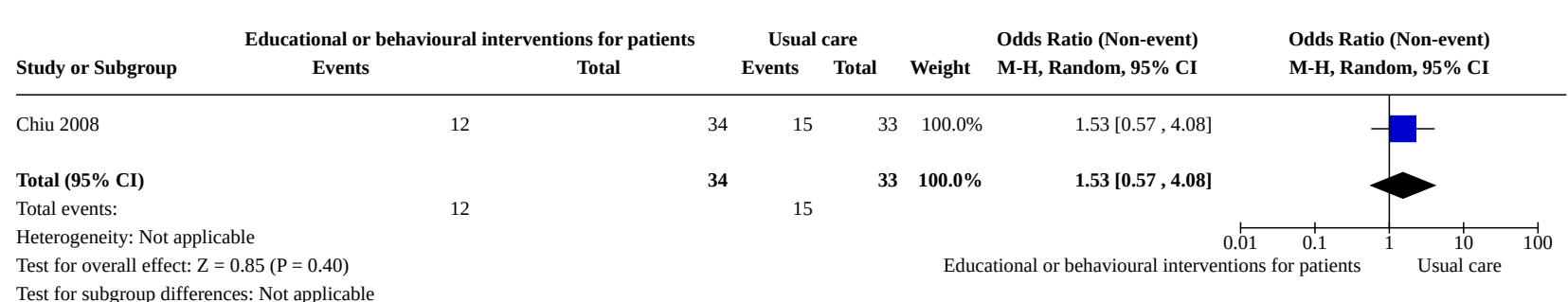

Test for subgroup differences: Not applicable

Analysis 1.11. Comparison 1: Educational or behavioural interventions for patients versus usual care, Outcome 11: Mean BMI

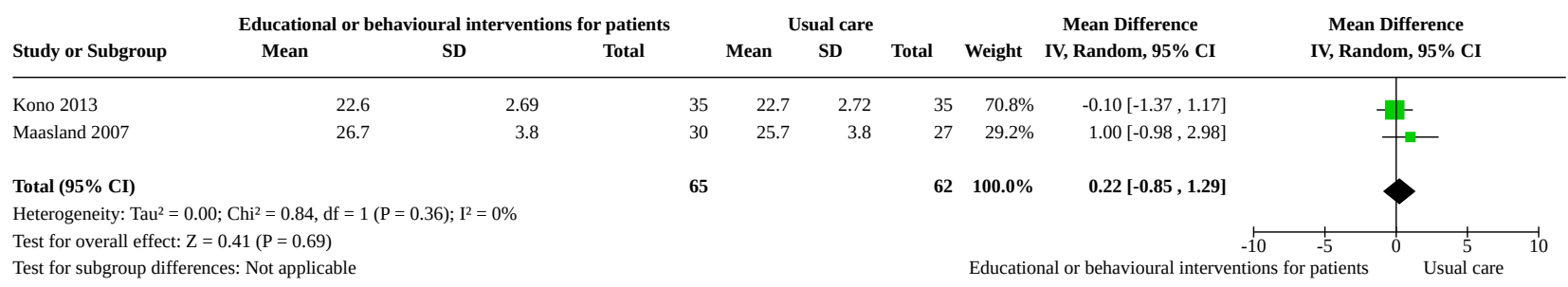

Analysis 1.12. Comparison 1: Educational or behavioural interventions for patients versus usual care, Outcome 12: Proporation of participants with secondary stroke

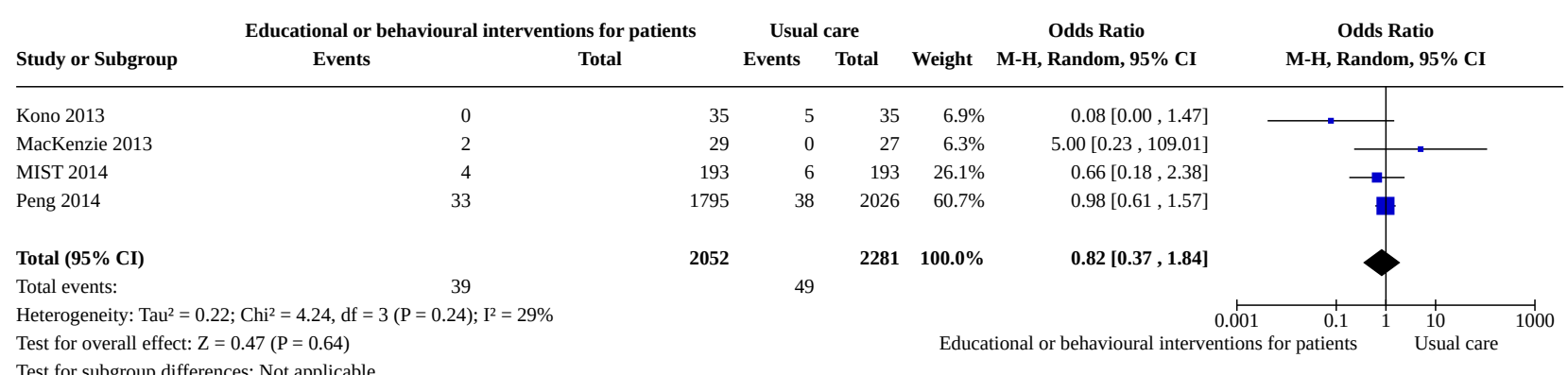


Analysis 1.13. Comparison 1: Educational or behavioural interventions for patients versus usual care, Outcome 13: Number of secondary TIAs

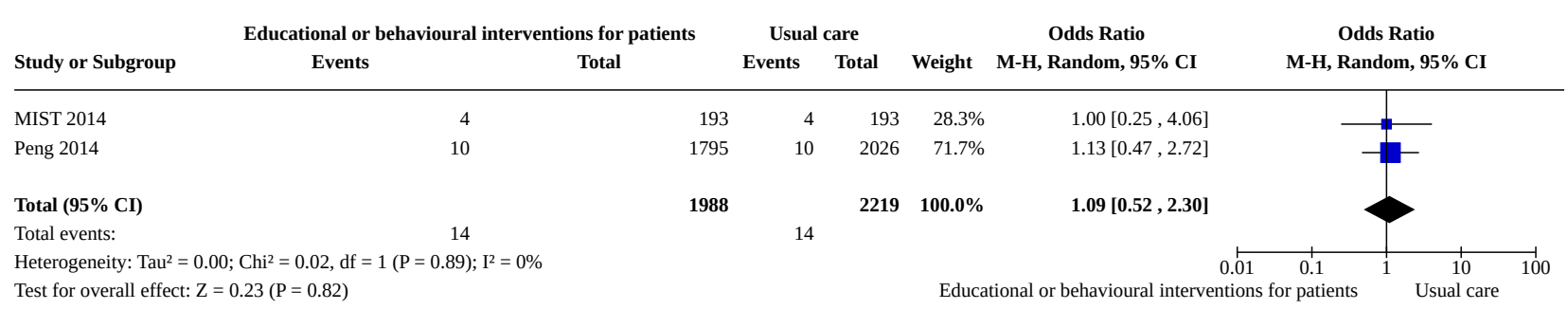

Test for overall effect. $Z=0.23(\mathrm{P}=0.82)$

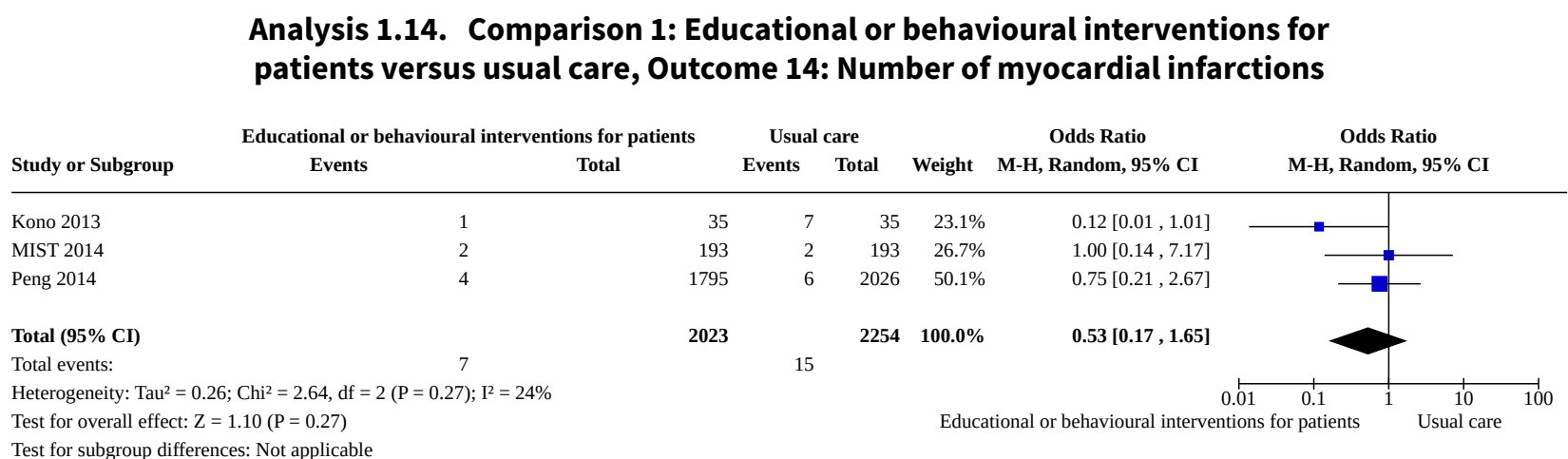

\section{Analysis 1.15. Comparison 1: Educational or behavioural interventions for} patients versus usual care, Outcome 15: Number of cardiovascular deaths

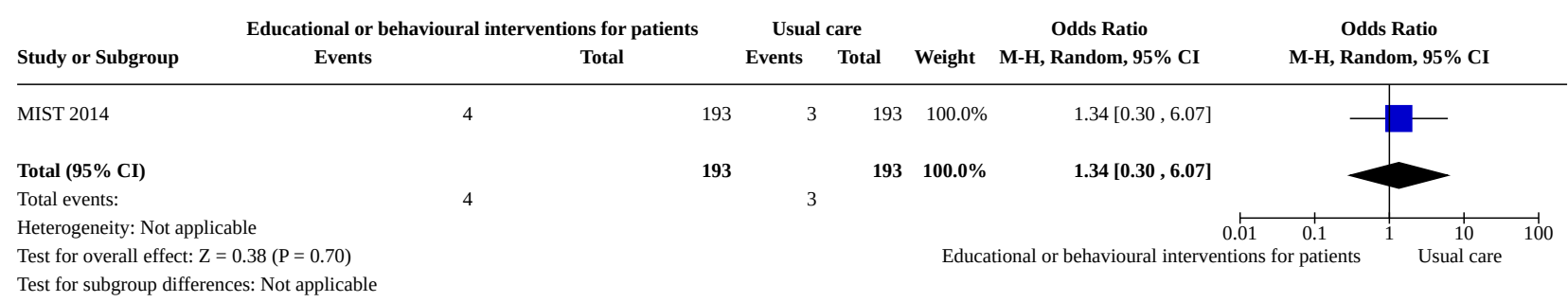

\section{Comparison 2. Organisational interventions versus usual care}

\begin{tabular}{|c|c|c|c|c|}
\hline Outcome or subgroup title & No. of studies & $\begin{array}{l}\text { No. of partici- } \\
\text { pants }\end{array}$ & Statistical method & Effect size \\
\hline 2.1 Mean systolic blood pressure & 16 & 17490 & $\begin{array}{l}\text { Mean Difference (IV, Random, } \\
95 \% \mathrm{CI} \text { ) }\end{array}$ & $-1.58[-4.66,1.51]$ \\
\hline 2.2 Mean diastolic blood pressure & 14 & 17178 & $\begin{array}{l}\text { Mean Difference (IV, Random, } \\
95 \% \mathrm{CI} \text { ) }\end{array}$ & $-0.91[-2.75,0.93]$ \\
\hline $\begin{array}{l}2.3 \text { Blood pressure target achieve- } \\
\text { ment }\end{array}$ & 13 & 23631 & $\begin{array}{l}\text { Odds Ratio (M-H, Random, } \\
95 \% \mathrm{Cl})\end{array}$ & $0.70[0.53,0.92]$ \\
\hline 2.4 Mean total cholesterol & 7 & 11955 & $\begin{array}{l}\text { Mean Difference (IV, Random, } \\
95 \% \mathrm{CI} \text { ) }\end{array}$ & $-0.00[-0.04,0.03]$ \\
\hline
\end{tabular}




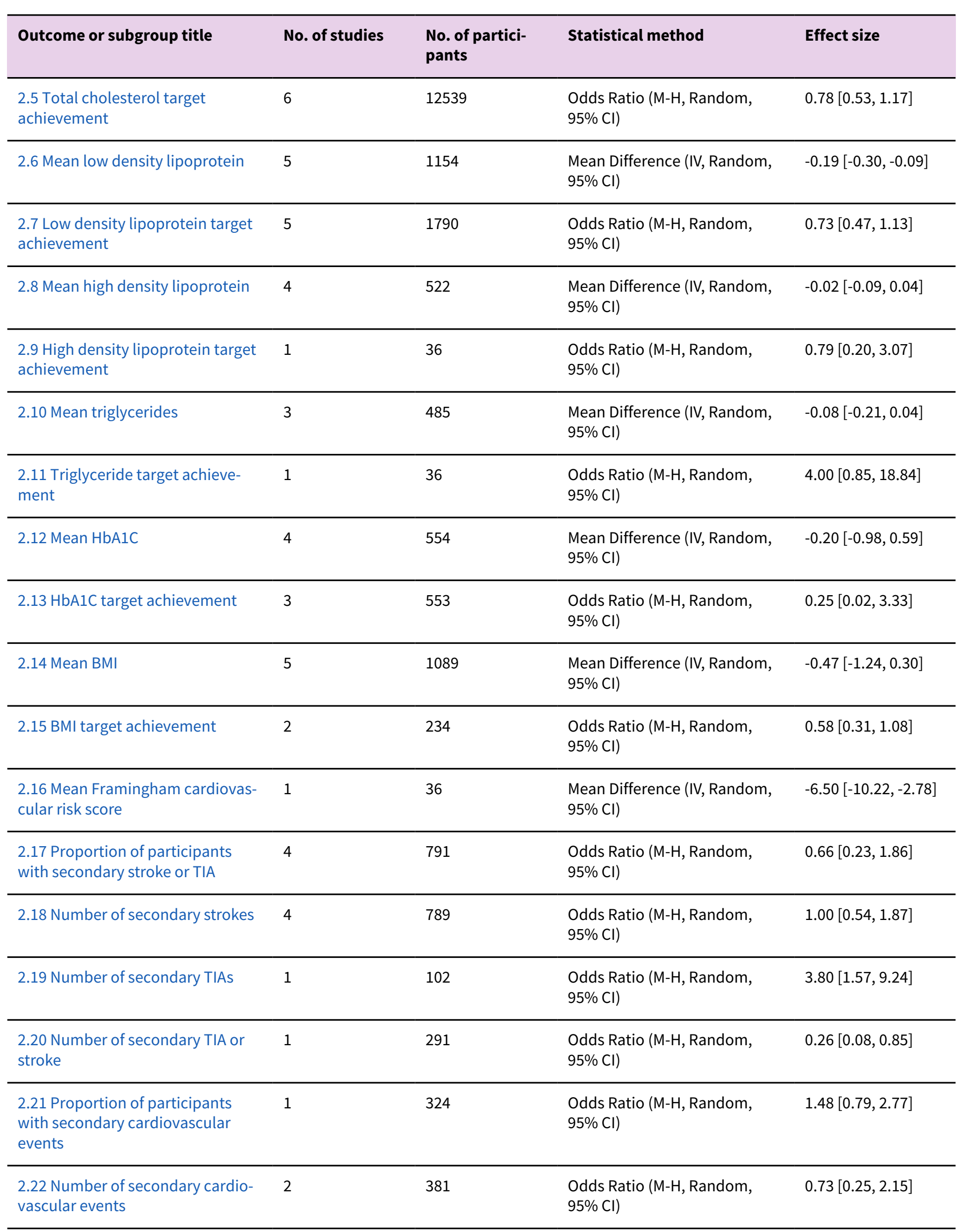




\begin{tabular}{lllll}
\hline Outcome or subgroup title & No. of studies & $\begin{array}{l}\text { No. of partici- } \\
\text { pants }\end{array}$ & Statistical method & Effect size \\
\hline $\begin{array}{l}2.23 \text { Number of myocardial infarc- } \\
\text { tions }\end{array}$ & 1 & 314 & $\begin{array}{l}\text { Odds Ratio (M-H, Random, } \\
95 \% \mathrm{Cl})\end{array}$ & $1.00[0.14,7.19]$ \\
\hline 2.24 Number of vascular deaths & 2 & 605 & $\begin{array}{l}\text { Odds Ratio (M-H, Random, } \\
95 \% \mathrm{Cl})\end{array}$ & $0.38[0.15,0.97]$ \\
\hline
\end{tabular}

Analysis 2.1. Comparison 2: Organisational interventions versus usual care, Outcome 1: Mean systolic blood pressure

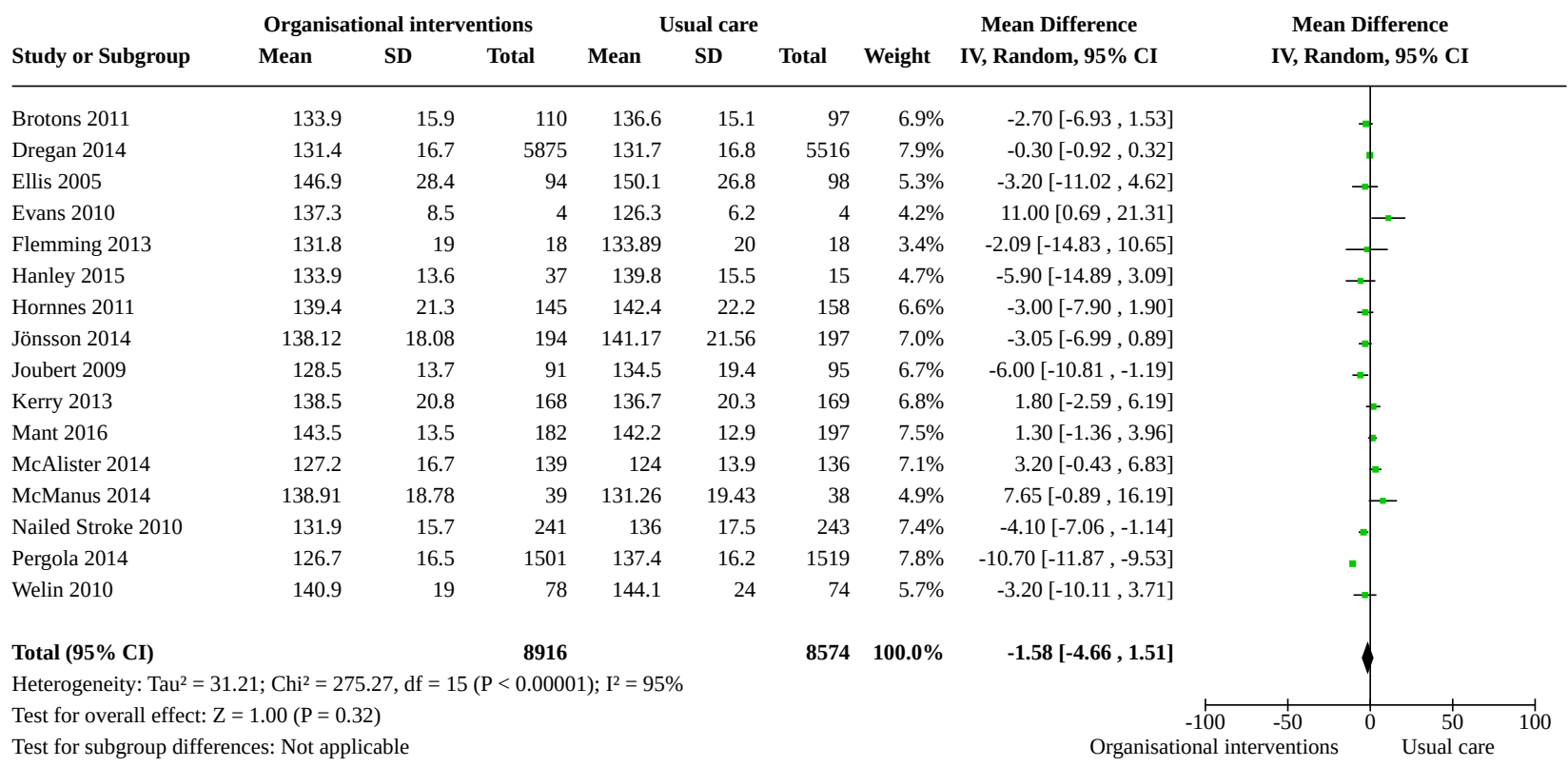


Analysis 2.2. Comparison 2: Organisational interventions versus usual care, Outcome 2: Mean diastolic blood pressure

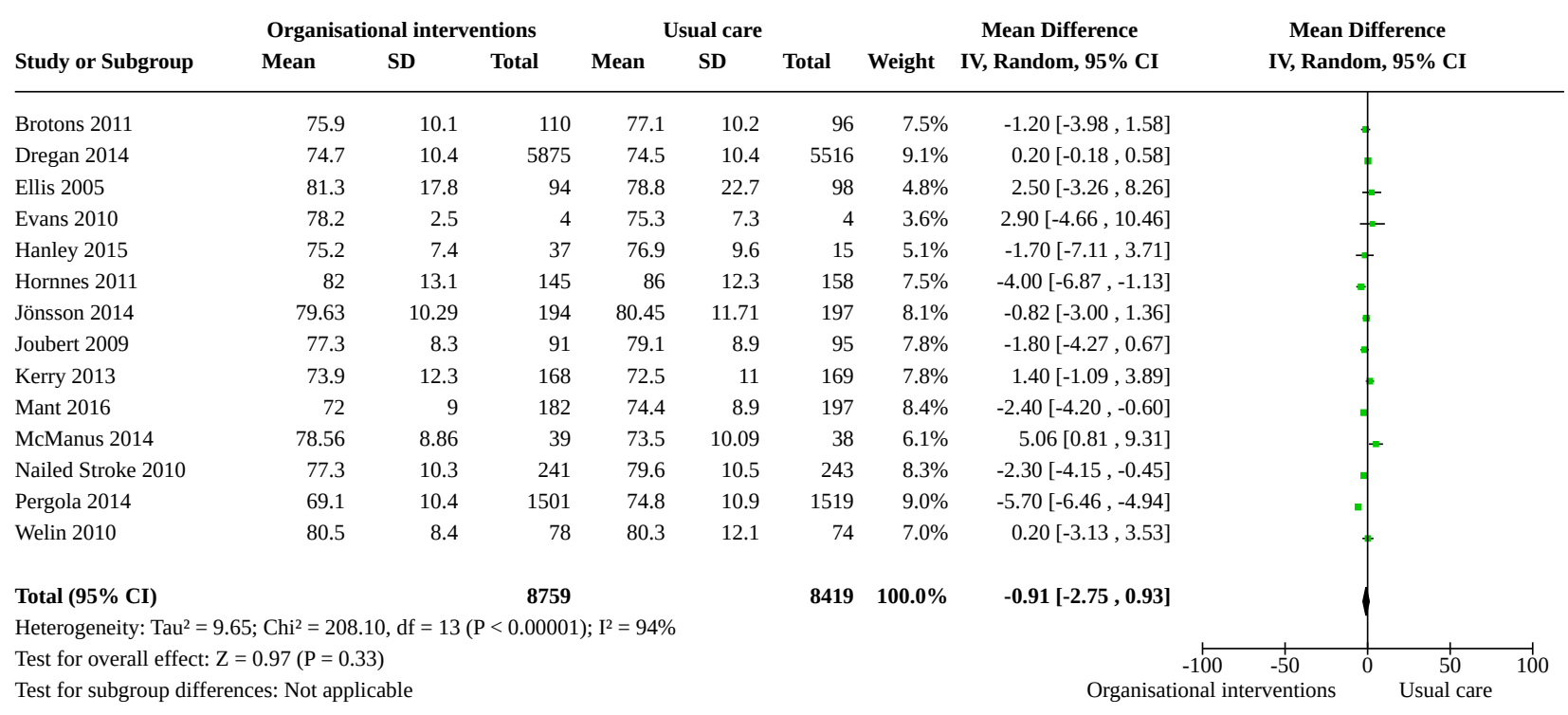

\section{Analysis 2.3. Comparison 2: Organisational interventions versus usual care, Outcome 3: Blood pressure target achievement}

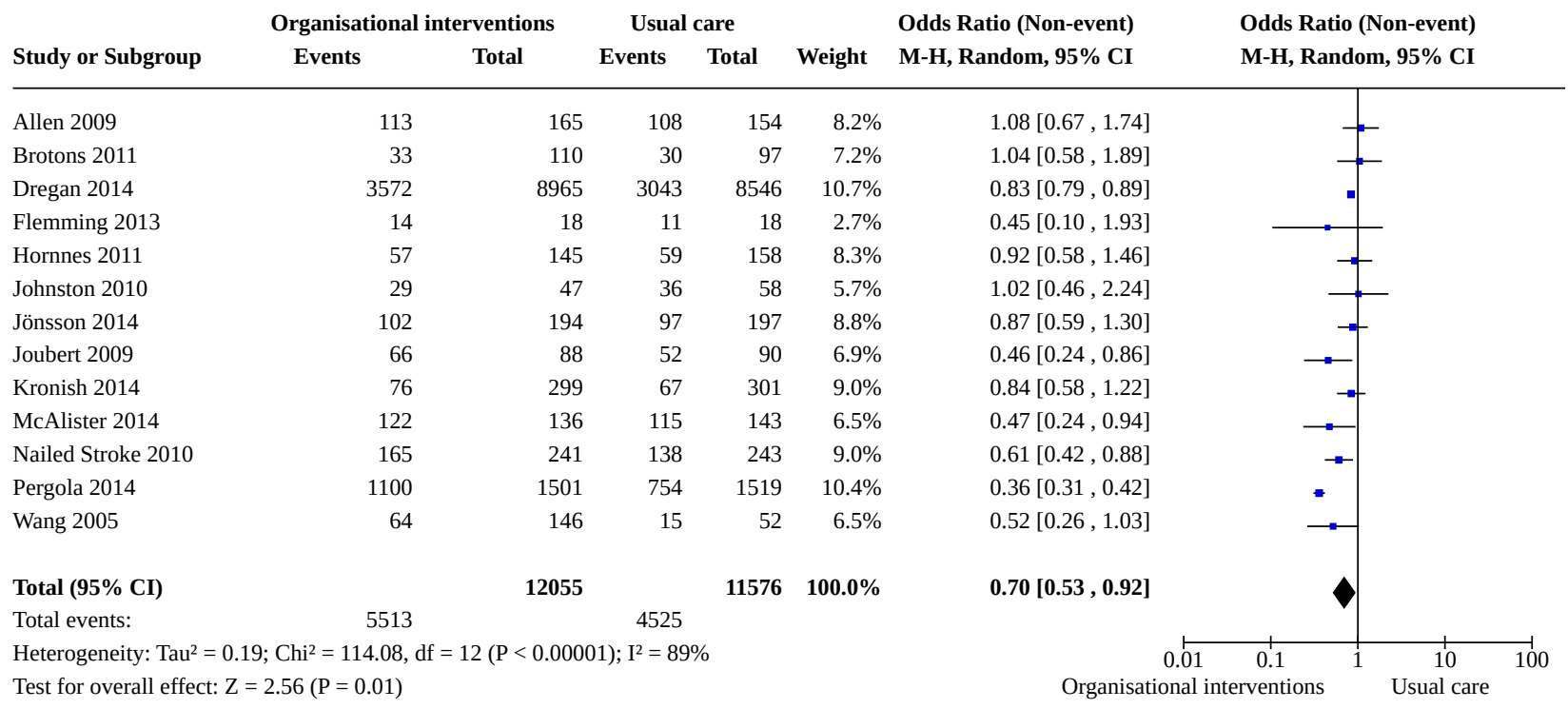


Analysis 2.4. Comparison 2: Organisational interventions versus usual care, Outcome 4: Mean total cholesterol

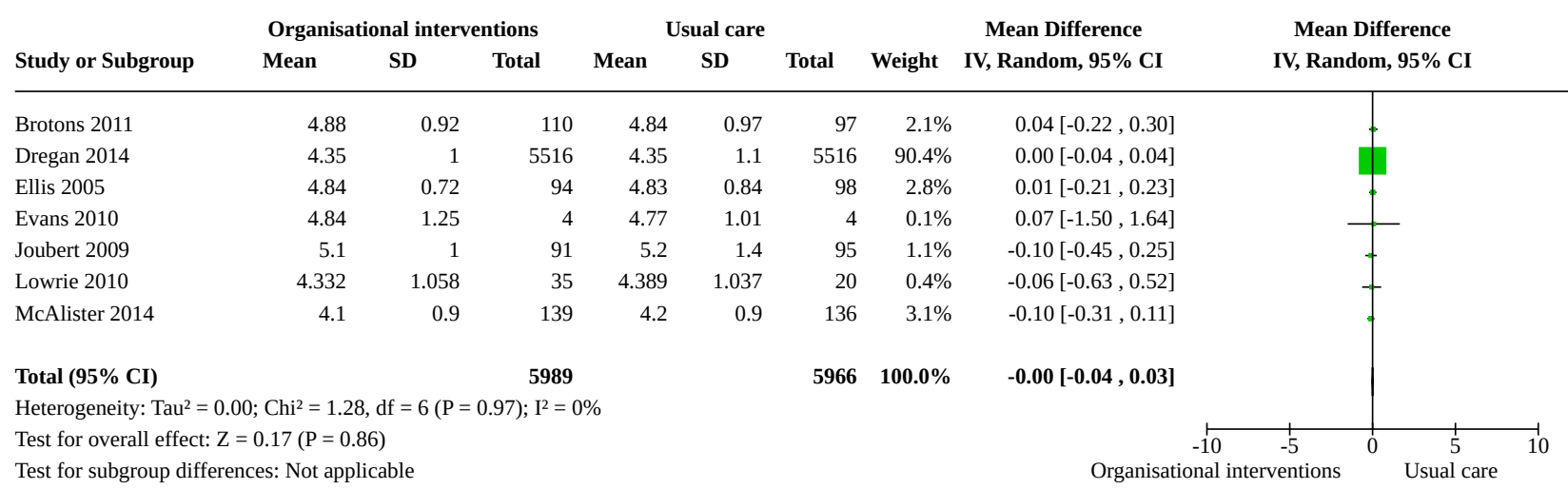

\section{Analysis 2.5. Comparison 2: Organisational interventions versus usual care, Outcome 5: Total cholesterol target achievement}

\begin{tabular}{|c|c|c|c|c|c|c|c|}
\hline \multirow[b]{2}{*}{ Study or Subgroup } & \multicolumn{2}{|c|}{ Organisational interventions } & \multicolumn{2}{|c|}{ Usual care } & \multirow[b]{2}{*}{ Weight } & \multirow{2}{*}{$\begin{array}{l}\text { Odds Ratio (Non-event) } \\
\text { M-H, Random, } 95 \% \text { CI }\end{array}$} & \multirow{2}{*}{$\begin{array}{l}\text { Odds Ratio (Non-event) } \\
\text { M-H, Random, 95\% CI }\end{array}$} \\
\hline & Events & Total & Events & Total & & & \\
\hline Allen 2009 & 107 & 165 & 107 & 154 & $18.2 \%$ & $1.23[0.77,1.97]$ & - \\
\hline Dregan 2014 & 2160 & 5875 & 2063 & 5516 & $24.0 \%$ & $1.03[0.95,1.11]$ & $\phi$ \\
\hline Jönsson 2014 & 125 & 194 & 127 & 197 & $19.2 \%$ & $1.00[0.66,1.52]$ & \\
\hline Joubert 2009 & 57 & 88 & 50 & 90 & $15.6 \%$ & $0.68[0.37,1.24]$ & $\rightarrow$ \\
\hline Lowrie 2010 & 13 & 39 & 8 & 23 & $8.7 \%$ & $1.07[0.36,3.16]$ & \\
\hline Wang 2005 & 109 & 146 & 19 & 52 & $14.3 \%$ & $0.20[0.10,0.38]$ & $\longrightarrow$ \\
\hline Total (95\% CI) & & 6507 & & 6032 & $100.0 \%$ & $0.78[0.53,1.17]$ & \\
\hline Total events: & 2571 & & 2374 & & & & \\
\hline Heterogeneity: $\mathrm{Tau}^{2}=$ & ; $\mathrm{Chi}^{2}=25.20, \mathrm{df}$ & $(P=0.0001)$ & $\mathrm{I}^{2}=80 \%$ & & & $\begin{array}{r}0.01 \\
\text { Organisational }\end{array}$ & $\begin{array}{ccc}0.1 & 1 & 10 \\
\end{array}$ \\
\hline
\end{tabular}

Test for subgroup differences: Not applicable

Analysis 2.6. Comparison 2: Organisational interventions versus usual care, Outcome 6: Mean low density lipoprotein

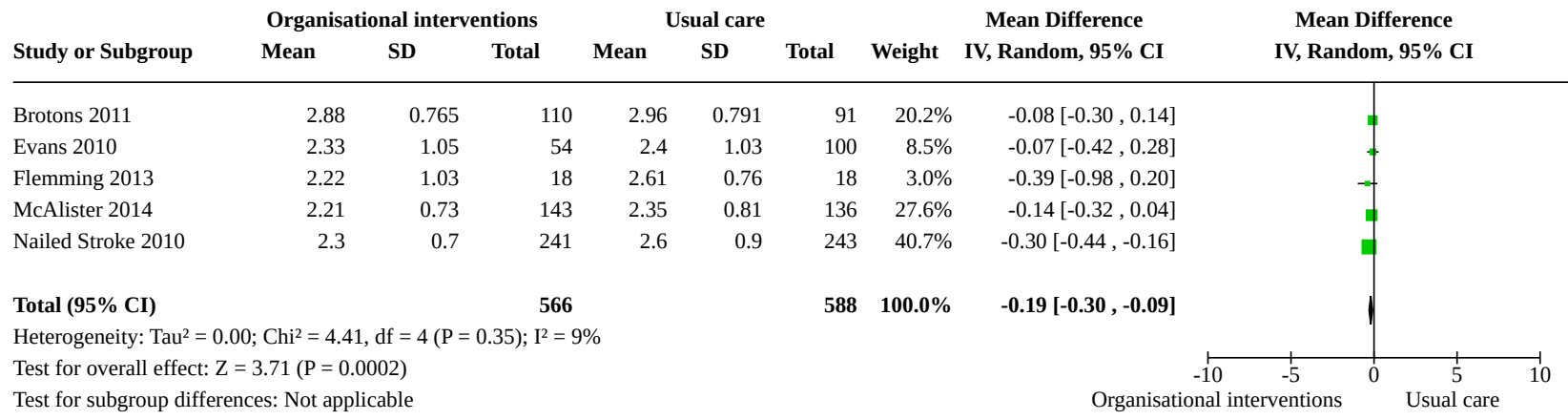


Analysis 2.7. Comparison 2: Organisational interventions versus
usual care, Outcome 7: Low density lipoprotein target achievement

\begin{tabular}{|c|c|c|c|c|c|c|c|}
\hline \multirow[b]{2}{*}{ r Subgroup } & \multicolumn{2}{|c|}{ Organisational interventions } & \multicolumn{3}{|c|}{ Usual care } & \multirow{2}{*}{$\begin{array}{l}\text { Odds Ratio (Non-event) } \\
\text { M-H, Random, 95\% CI }\end{array}$} & \multirow{2}{*}{$\begin{array}{l}\text { Odds Ratio (Non-event) } \\
\text { M-H, Random, } 95 \% \text { CI }\end{array}$} \\
\hline & Events & Total & Events & Total & Weight & & \\
\hline
\end{tabular}

\begin{tabular}{|c|c|c|c|c|c|c|c|c|}
\hline Flemming 2013 & 10 & 18 & 10 & 18 & $8.1 \%$ & $1.00[0.27,3.72]$ & $\longrightarrow$ & \\
\hline Jönsson 2014 & 72 & 194 & 75 & 197 & $23.2 \%$ & $1.04[0.69,1.57]$ & & - \\
\hline Kronish 2014 & 54 & 299 & 58 & 301 & $23.2 \%$ & $1.08[0.72,1.63]$ & & 1 \\
\hline McAlister 2014 & 73 & 143 & 46 & 136 & $21.5 \%$ & $0.49[0.30,0.79]$ & $\rightarrow$ & \\
\hline Nailed Stroke 2010 & 161 & 241 & 115 & 243 & $24.1 \%$ & $0.45[0.31,0.64]$ & $\rightarrow$ & \\
\hline Total (95\% CI) & & 895 & & 895 & $100.0 \%$ & $0.73[0.47,1.13]$ & & \\
\hline Total events: & 370 & & 304 & & & & & \\
\hline \multicolumn{6}{|c|}{ Heterogeneity: $\mathrm{Tau}^{2}=0.17 ; \mathrm{Chi}^{2}=16.05, \mathrm{df}=4(\mathrm{P}=0.003) ; \mathrm{I}^{2}=75 \%$} & 0.01 & 0.1 & 10 \\
\hline
\end{tabular}

Test for subgroup differences: Not applicable

Analysis 2.8. Comparison 2: Organisational interventions versus usual care, Outcome 8: Mean high density lipoprotein

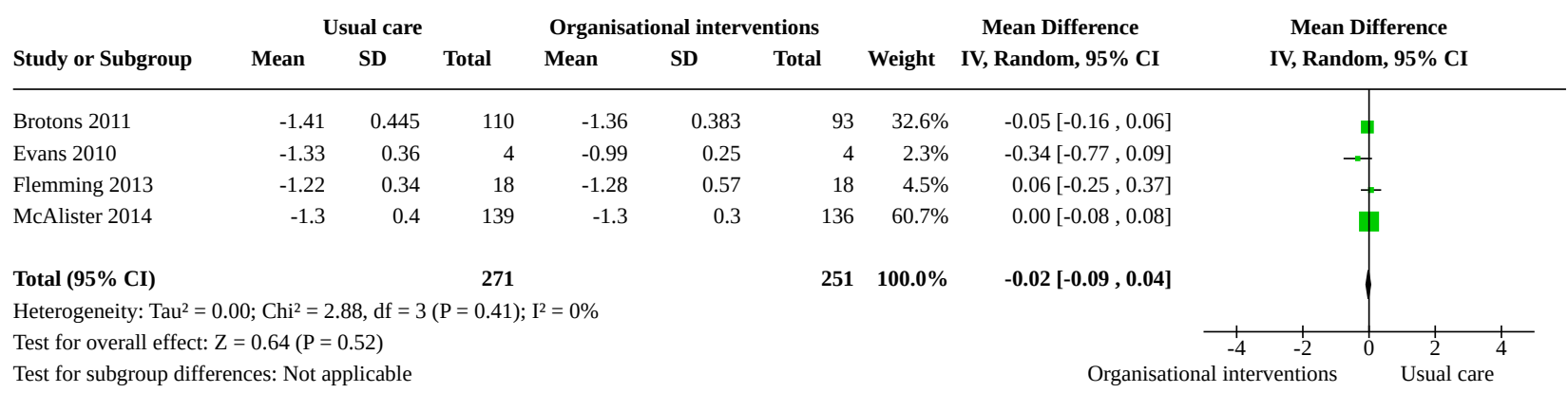
Analysis 2.9. Comparison 2: Organisational interventions versus
usual care, Outcome 9: High density lipoprotein target achievement

\begin{tabular}{|c|c|c|c|c|c|c|c|}
\hline \multirow[b]{2}{*}{ Study or Subgroup } & \multicolumn{2}{|c|}{ Organisational interventions } & \multicolumn{2}{|c|}{ Usual care } & \multirow[b]{2}{*}{ Weight } & \multirow{2}{*}{$\begin{array}{l}\text { Odds Ratio (Non-event) } \\
\text { M-H, Random, 95\% CI }\end{array}$} & \multirow{2}{*}{$\begin{array}{l}\text { Odds Ratio (Non-event) } \\
\text { M-H, Random, 95\% CI }\end{array}$} \\
\hline & Events & Total & Events & Total & & & \\
\hline Flemming 2013 & 12 & 18 & 11 & 18 & $100.0 \%$ & $0.79[0.20,3.07]$ & \\
\hline Total (95\% CI) & & 18 & & 18 & $100.0 \%$ & $0.79[0.20,3.07]$ & \\
\hline Total events: & 12 & & 11 & & & & \\
\hline Heterogeneity: Not a & able & & & & & 0.01 & $1 \quad 10$ \\
\hline
\end{tabular}


Analysis 2.10. Comparison 2: Organisational interventions versus usual care, Outcome 10: Mean triglycerides

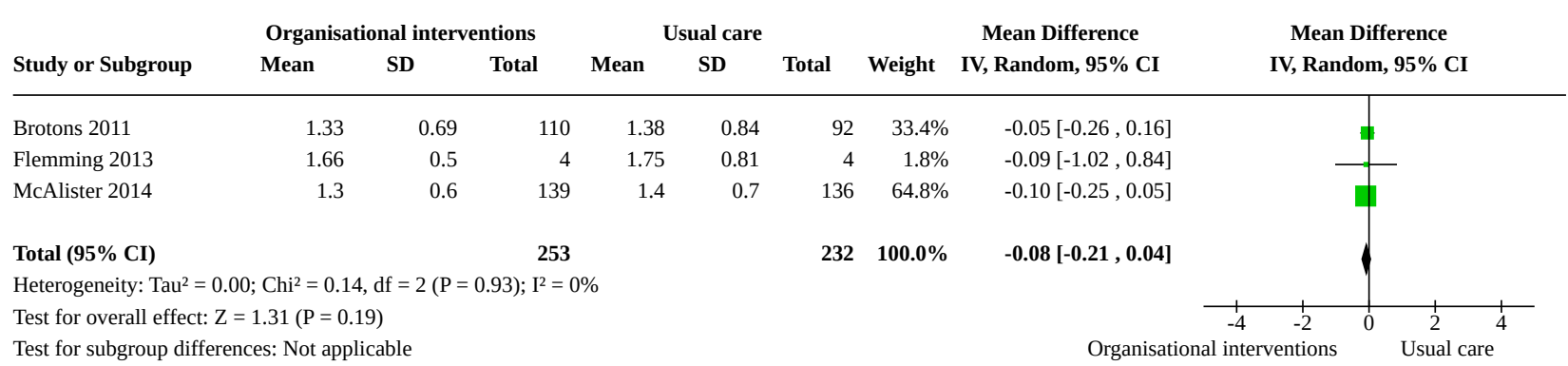

Analysis 2.11. Comparison 2: Organisational interventions versus usual care, Outcome 11: Triglyceride target achievement

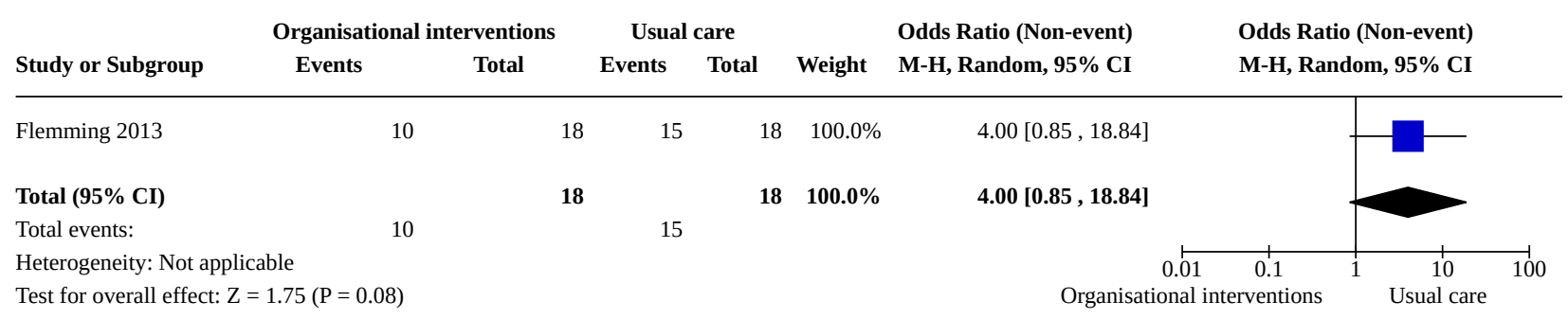

Test for subgroup differences: Not applicable

\section{Analysis 2.12. Comparison 2: Organisational interventions versus usual care, Outcome 12: Mean HbA1C}

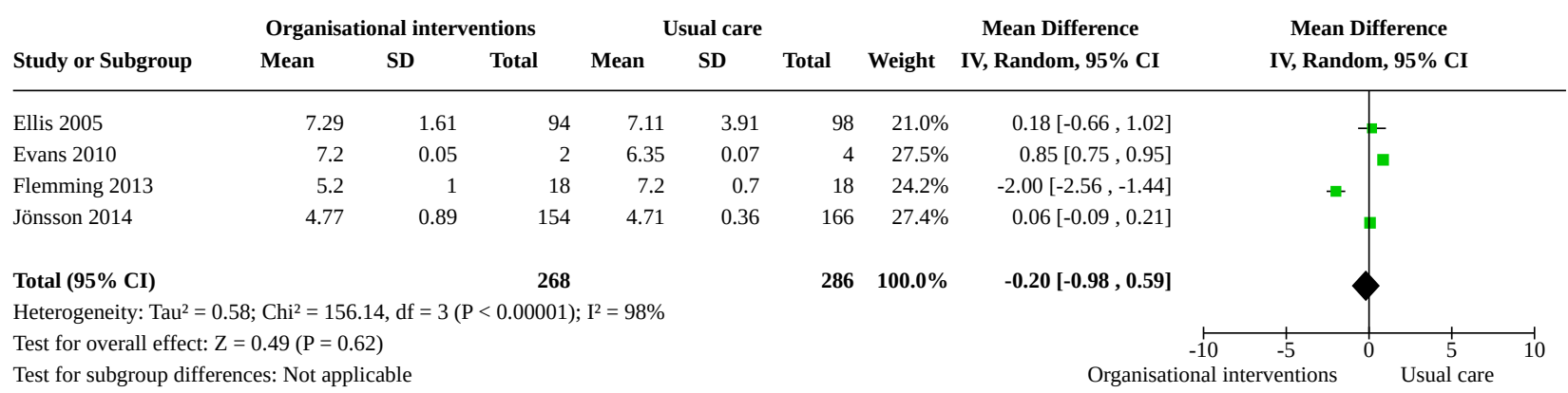

\section{Analysis 2.13. Comparison 2: Organisational interventions versus usual care, Outcome 13: HbA1C target achievement}

\begin{tabular}{|c|c|c|c|c|c|c|c|c|}
\hline \multirow[b]{2}{*}{ Study or Subgroup } & \multicolumn{2}{|c|}{ Organisational interventions } & \multicolumn{2}{|c|}{ Usual care } & \multirow[b]{2}{*}{ Weight } & \multirow{2}{*}{$\begin{array}{l}\text { Odds Ratio (Non-event) } \\
\text { M-H, Random, 95\% CI }\end{array}$} & \multirow{2}{*}{$\begin{array}{l}\text { Odds Ratio (Non-event) } \\
\text { M-H, Random, 95\% CI }\end{array}$} & \\
\hline & Events & Total & Events & Total & & & & \\
\hline Allen 2009 & 118 & 165 & 119 & 154 & $38.0 \%$ & $1.35[0.82,2.25]$ & & \\
\hline Flemming 2013 & 18 & 18 & 16 & 18 & $24.7 \%$ & $0.18[0.01,3.99]$ & & \\
\hline Wang 2005 & 129 & 146 & 16 & 52 & $37.3 \%$ & $0.06[0.03,0.13]$ & & \\
\hline Total (95\% CI) & & 329 & & 224 & $100.0 \%$ & $0.25[0.02,3.33]$ & & \\
\hline Total events: & 265 & & 151 & & & & & \\
\hline \multicolumn{6}{|c|}{ Heterogeneity: $\mathrm{Tau}^{2}=4.45 ; \mathrm{Chi}^{2}=44.60, \mathrm{df}=2(\mathrm{P}<0.00001) ; \mathrm{I}^{2}=96 \%$} & 0.001 & 0.1 & 1000 \\
\hline \multicolumn{4}{|c|}{ Test for overall effect: $\mathrm{Z}=1.04(\mathrm{P}=0.30)$} & & & Organisational i & erventions & \\
\hline
\end{tabular}




\section{Analysis 2.14. Comparison 2: Organisational interventions versus usual care, Outcome 14: Mean BMI}

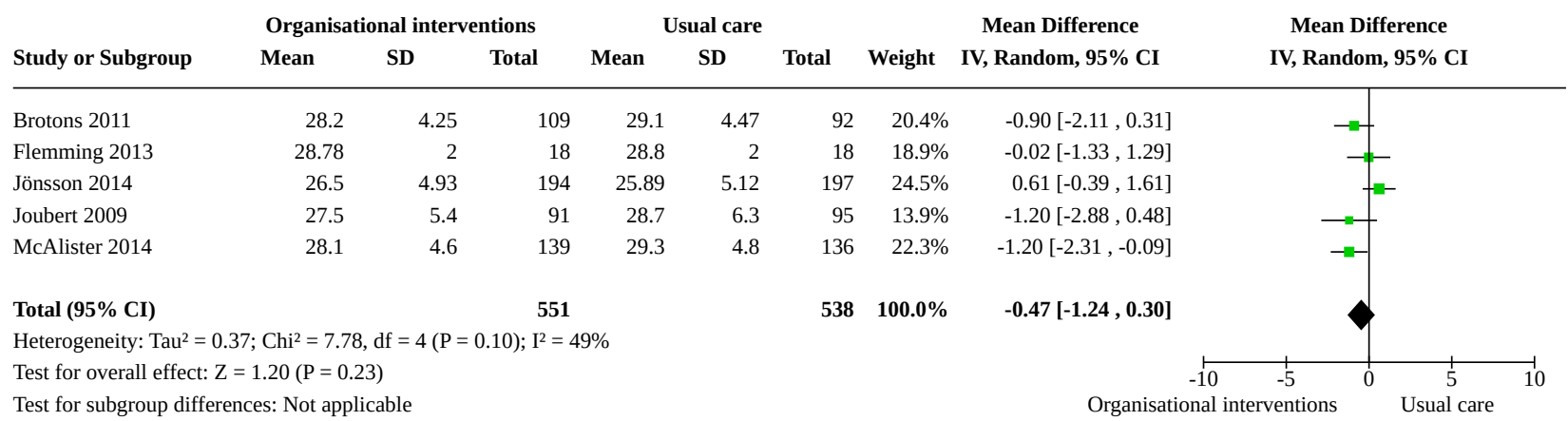

Analysis 2.15. Comparison 2: Organisational interventions versus usual care, Outcome 15: BMI target achievement

\begin{tabular}{|c|c|c|c|c|c|}
\hline & Organisational interventions & Usual care & & Odds Ratio (Non-event) & Odds Ratio (Non-event) \\
\hline Study or Subgroup & Events & Events & Weight & M-H, Random, 95\% CI & M-H, Random, 95\% CI \\
\hline
\end{tabular}

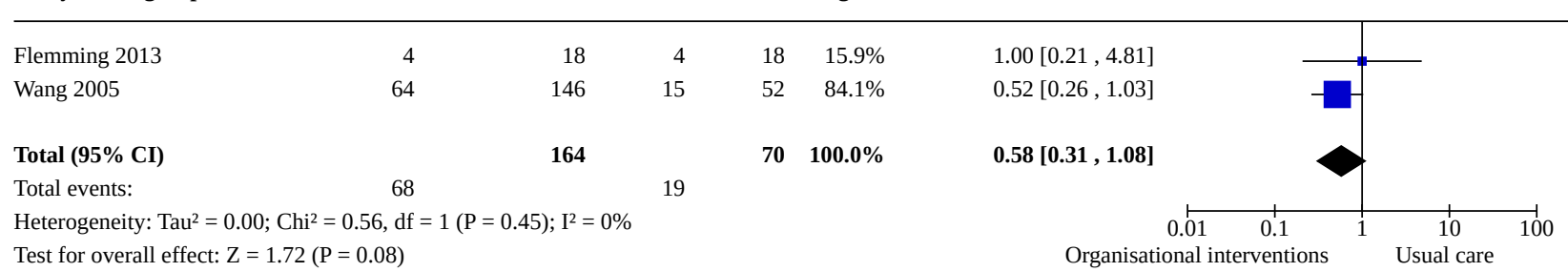

Test for subgroup differences: Not applicable

Analysis 2.16. Comparison 2: Organisational interventions versus usual care, Outcome 16: Mean Framingham cardiovascular risk score

\begin{tabular}{|c|c|c|c|c|c|c|c|c|c|c|c|}
\hline \multirow[b]{2}{*}{ Study or Subgroup } & \multicolumn{3}{|c|}{ Organisational interventions } & \multicolumn{3}{|c|}{ Usual care } & \multicolumn{2}{|r|}{ Mean Difference } & \multirow{2}{*}{\multicolumn{2}{|c|}{$\begin{array}{c}\text { Mean Difference } \\
\text { IV, Random, 95\% CI }\end{array}$}} & \\
\hline & Mean & SD & Total & Mean & SD & Total & Weight & IV, Random, 95\% CI & & & \\
\hline Flemming 2013 & -5.2 & 7 & 18 & 1.3 & 4 & 18 & $100.0 \%$ & $-6.50[-10.22,-2.78]$ & & & \\
\hline Total $(95 \%$ CI) & & & 18 & & & 18 & $100.0 \%$ & $-6.50[-10.22,-2.78]$ & $\Delta$ & & \\
\hline \multicolumn{12}{|c|}{ Heterogeneity: Not applicable } \\
\hline \multicolumn{4}{|c|}{ Test for overall effect: $\mathrm{Z}=3.42(\mathrm{P}=0.0006)$} & & & & & -100 & -50 & 50 & 100 \\
\hline Test for subgroup dif & es: Not app & able & & & & & & Organisational i & rventions & Usual ca & \\
\hline
\end{tabular}


Analysis 2.17. Comparison 2: Organisational interventions versus usual care, Outcome 17: Proportion of participants with secondary stroke or TIA

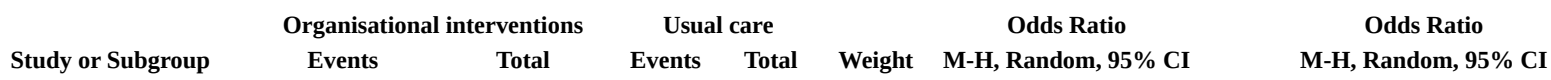

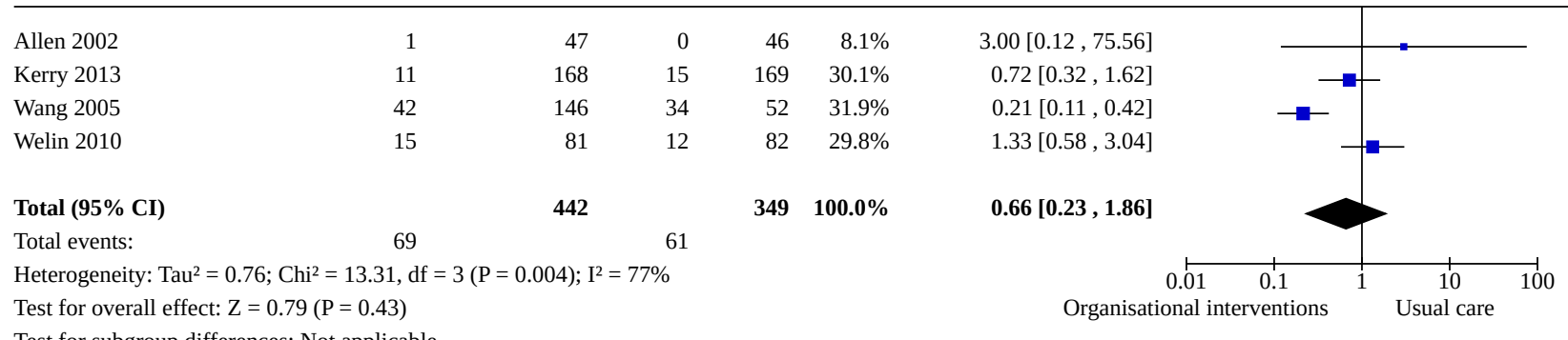

Test for subgroup differences: Not applicable

Analysis 2.18. Comparison 2: Organisational interventions versus usual care, Outcome 18: Number of secondary strokes

\begin{tabular}{|c|c|c|c|c|c|c|c|}
\hline \multirow[b]{2}{*}{ Study or Subgroup } & \multicolumn{2}{|c|}{ Organisational interventions } & \multicolumn{2}{|c|}{ Usual care } & \multirow[b]{2}{*}{ Weight } & \multirow{2}{*}{$\begin{array}{c}\text { Odds Ratio } \\
\text { M-H, Random, 95\% CI }\end{array}$} & \multirow{2}{*}{$\begin{array}{c}\text { Odds Ratio } \\
\text { M-H, Random, 95\% CI }\end{array}$} \\
\hline & Events & Total & Events & Total & & & \\
\hline Boysen 2009 & 14 & 157 & 11 & 157 & $46.5 \%$ & $1.30[0.57,2.96]$ & - \\
\hline Ellis 2005 & 2 & 49 & 3 & 53 & $11.0 \%$ & $0.71[0.11,4.43]$ & $\rightarrow$ \\
\hline Markle-Reid 2011 & 10 & 43 & 7 & 39 & $29.2 \%$ & $1.39[0.47,4.08]$ & $=$ \\
\hline Ranta 2015 & 2 & 172 & 5 & 119 & $13.3 \%$ & $0.27[0.05,1.41]$ & - \\
\hline Total (95\% CI) & & 421 & & 368 & $100.0 \%$ & $1.00[0.54,1.87]$ & \\
\hline Total events: & 28 & & 26 & & & & \\
\hline $\begin{array}{l}\text { Heterogeneity: } \mathrm{Tau}^{2}= \\
\text { Test for overall effect }\end{array}$ & $\begin{array}{l}; \mathrm{Chi}^{2}=3.29, \mathrm{df}= \\
0.01(\mathrm{P}=0.99)\end{array}$ & $\mathrm{P}=0.35) ; \mathrm{I}^{2}=$ & $9 \%$ & & & $\begin{array}{r}0.01 \\
\text { Organisational }\end{array}$ & $\begin{array}{lc}1 & 10 \\
& \text { Usual }\end{array}$ \\
\hline
\end{tabular}

Test for subgroup differences: Not applicable

Analysis 2.19. Comparison 2: Organisational interventions versus usual care, Outcome 19: Number of secondary TIAs

\begin{tabular}{|c|c|c|c|c|c|c|c|c|}
\hline \multirow[b]{2}{*}{ Study or Subgroup } & \multicolumn{2}{|c|}{ Organisational interventions } & \multicolumn{2}{|c|}{ Usual care } & \multirow[b]{2}{*}{ Weight } & \multirow{2}{*}{$\begin{array}{c}\text { Odds Ratio } \\
\text { M-H, Random, 95\% CI }\end{array}$} & \multirow{2}{*}{\multicolumn{2}{|c|}{$\begin{array}{c}\text { Odds Ratio } \\
\text { M-H, Random, 95\% CI }\end{array}$}} \\
\hline & Events & Total & Events & Total & & & & \\
\hline Ellis 2005 & 23 & 49 & 10 & 53 & $100.0 \%$ & $3.80[1.57,9.24]$ & & \\
\hline Total (95\% CI) & & 49 & & 53 & $100.0 \%$ & $3.80[1.57,9.24]$ & & \\
\hline Total events: & 23 & & 10 & & & & & \\
\hline \multicolumn{3}{|c|}{ Heterogeneity: Not applicable } & & & & 0.01 & 0.1 & 10 \\
\hline Test for overall effec & $2.95(\mathrm{P}=0.003)$ & & & & & Organisational i & erventions & Usual care \\
\hline
\end{tabular}




\section{Analysis 2.20. Comparison 2: Organisational interventions versus} usual care, Outcome 20: Number of secondary TIA or stroke

\begin{tabular}{|c|c|c|c|c|c|c|c|}
\hline & Organisa & rvention & Usu & care & & Odds Ratio & Odds Ratio \\
\hline Study or Subgroup & Events & Total & Events & Total & Weight & M-H, Random, 95\% CI & M-H, Random, 95\% CI \\
\hline
\end{tabular}

\begin{tabular}{|c|c|c|c|c|c|c|c|c|}
\hline Ranta 2015 & 4 & 172 & 10 & 119 & $100.0 \%$ & $0.26[0.08,0.85]$ & & \\
\hline Total (95\% CI) & & 172 & & 119 & $100.0 \%$ & $0.26[0.08,0.85]$ & & \\
\hline Total events: & 4 & & 10 & & & & & \\
\hline Heterogeneity: Not applicable & & & & & & 0.01 & 0.1 & $\begin{array}{ccc}1 & 10 & 100\end{array}$ \\
\hline
\end{tabular}

Test for subgroup differences: Not applicable

Analysis 2.21. Comparison 2: Organisational interventions versus usual care, Outcome 21: Proportion of participants with secondary cardiovascular events

\begin{tabular}{|c|c|c|c|c|c|c|c|c|c|}
\hline \multirow{3}{*}{$\begin{array}{l}\text { Study or Subgroup } \\
\text { Brotons } 2011\end{array}$} & \multicolumn{2}{|c|}{ Organisational intervention } & \multicolumn{2}{|c|}{ Usual care } & \multirow[b]{2}{*}{ Weight } & \multirow{2}{*}{$\begin{array}{c}\text { Odds Ratio } \\
\text { M-H, Random, 95\% CI }\end{array}$} & \multirow{2}{*}{\multicolumn{2}{|c|}{$\begin{array}{c}\text { Odds Ratio } \\
\text { M-H, Random, 95\% CI }\end{array}$}} & \\
\hline & Events & Total & Events & Total & & & & & \\
\hline & 27 & 159 & 20 & 165 & $100.0 \%$ & $1.48[0.79,2.77]$ & & & \\
\hline Total (95\% CI) & & 159 & & 165 & $100.0 \%$ & $1.48[0.79,2.77]$ & & & \\
\hline Total events: & 27 & & 20 & & & & & & \\
\hline Heterogeneity: Not a & ble & & & & & 0.01 & 0.1 & 10 & 100 \\
\hline Test for overall effec & $1.24(\mathrm{P}=0.22)$ & & & & & Organisational & ntervention & Usual care & \\
\hline
\end{tabular}

Test for subgroup differences: Not applicable

Analysis 2.22. Comparison 2: Organisational interventions versus usual care, Outcome 22: Number of secondary cardiovascular events

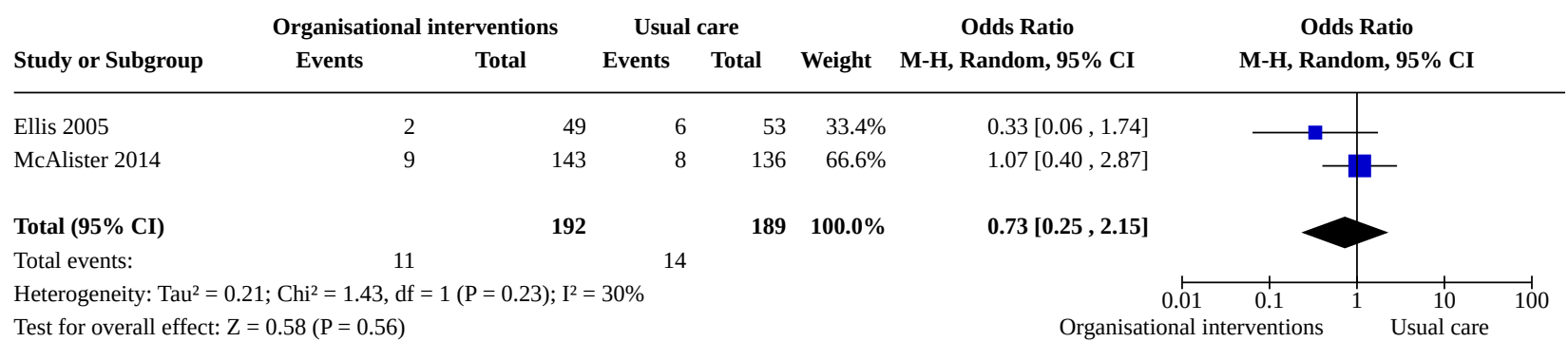

Test for overall effect: $Z=0.58(\mathrm{P}=0.56)$

Test for subgroup differences: Not applicable

Analysis 2.23. Comparison 2: Organisational interventions versus usual care, Outcome 23: Number of myocardial infarctions

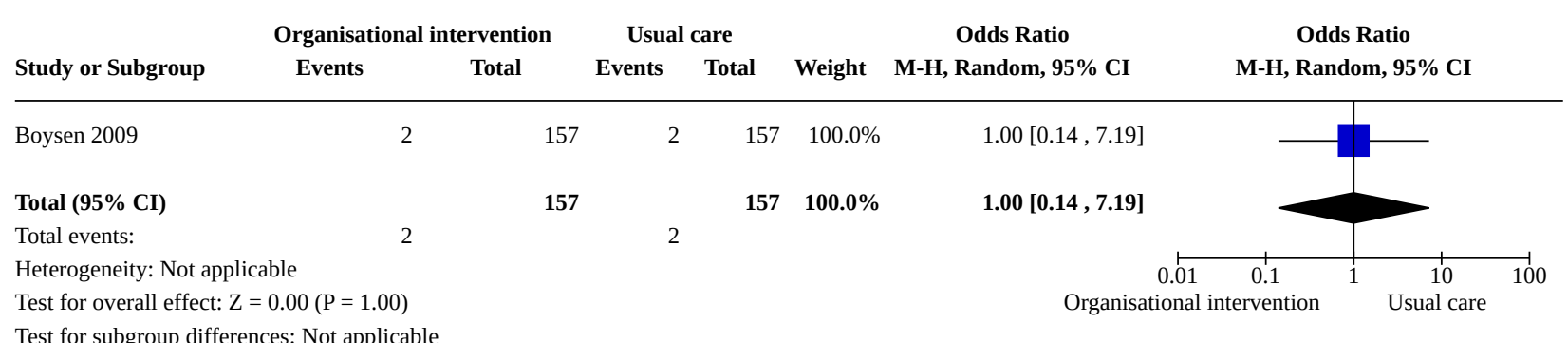


Analysis 2.24. Comparison 2: Organisational interventions versus usual care, Outcome 24: Number of vascular deaths

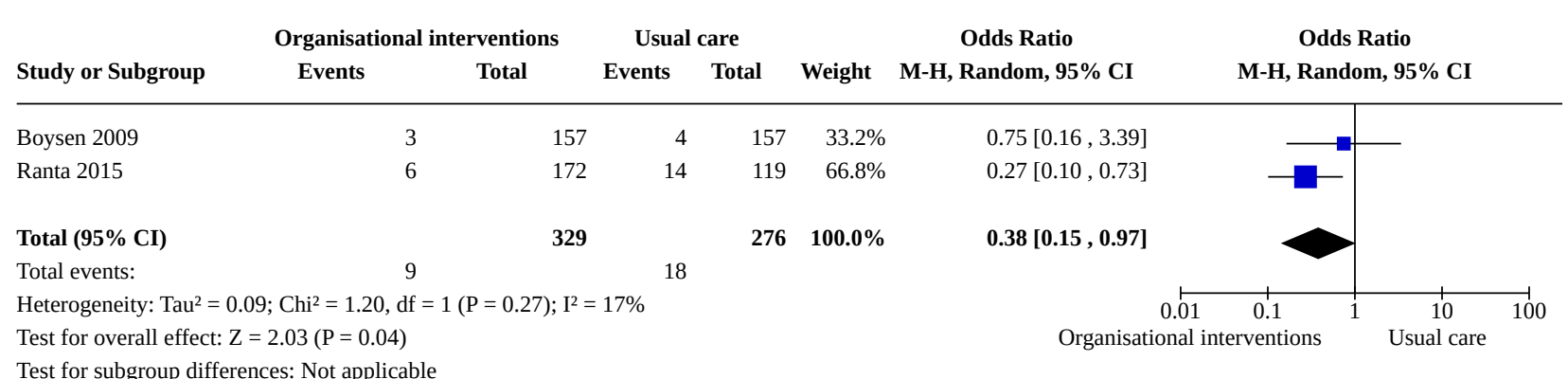

Test for subgroup differences: Not applicable 


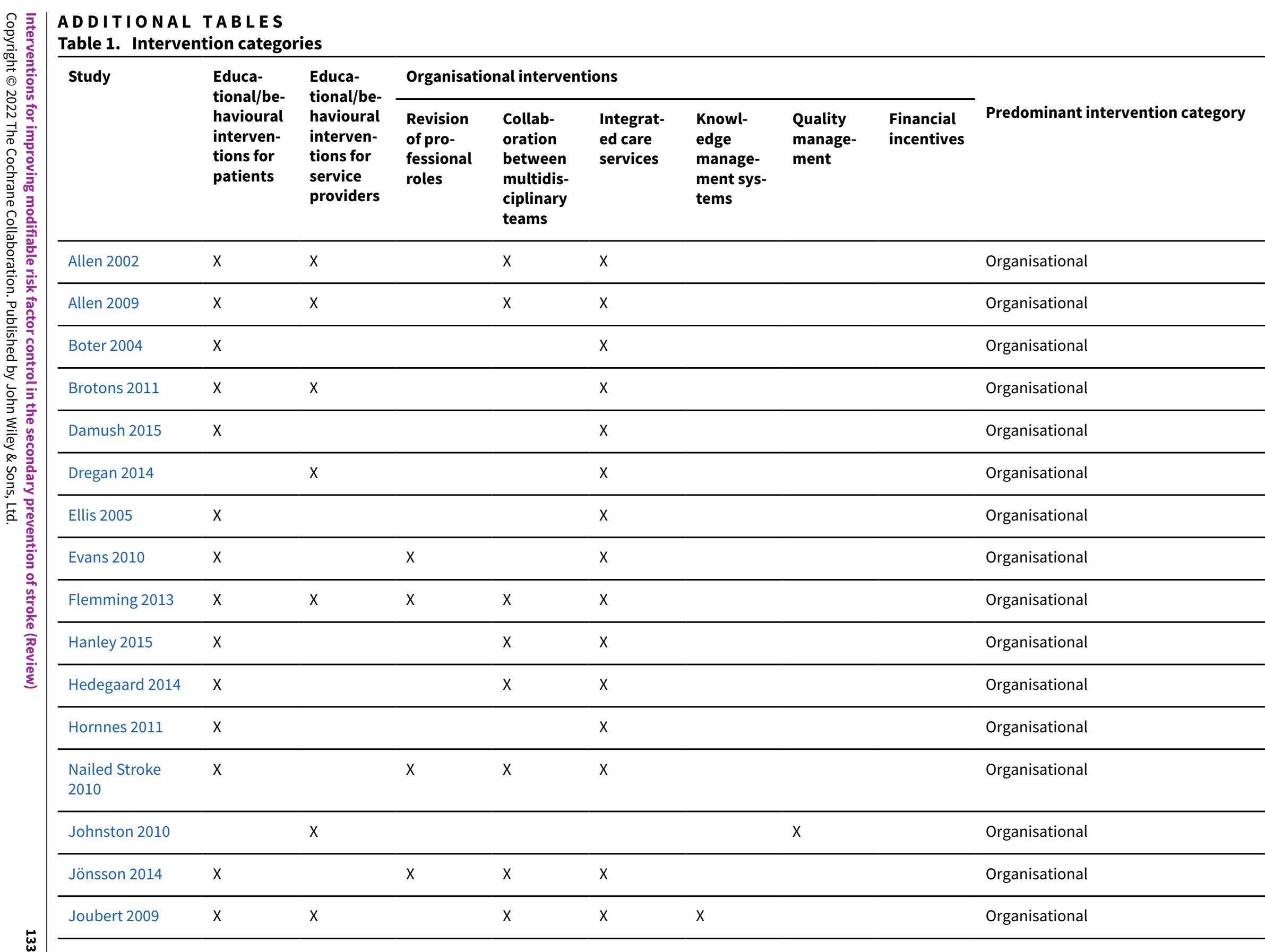




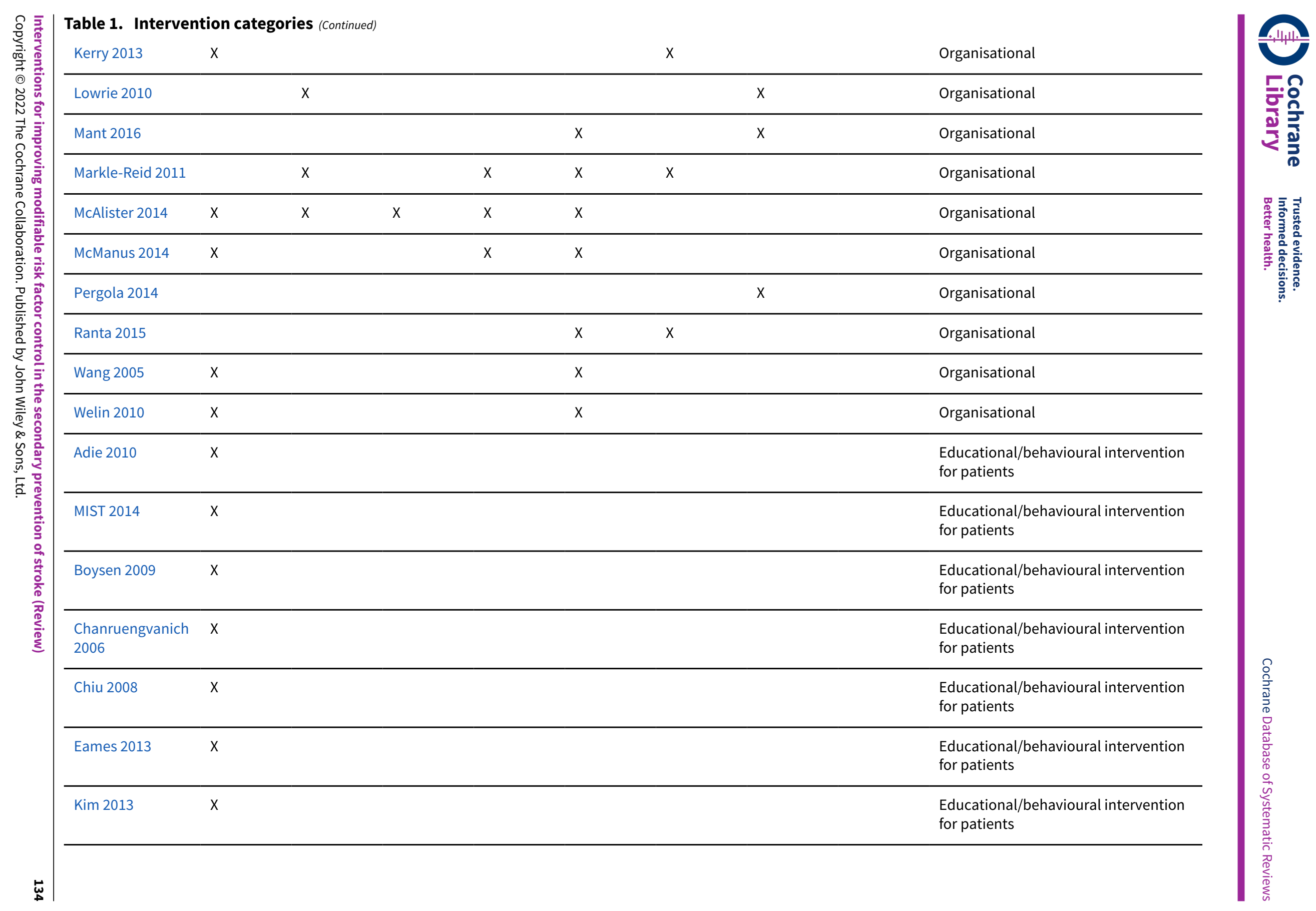




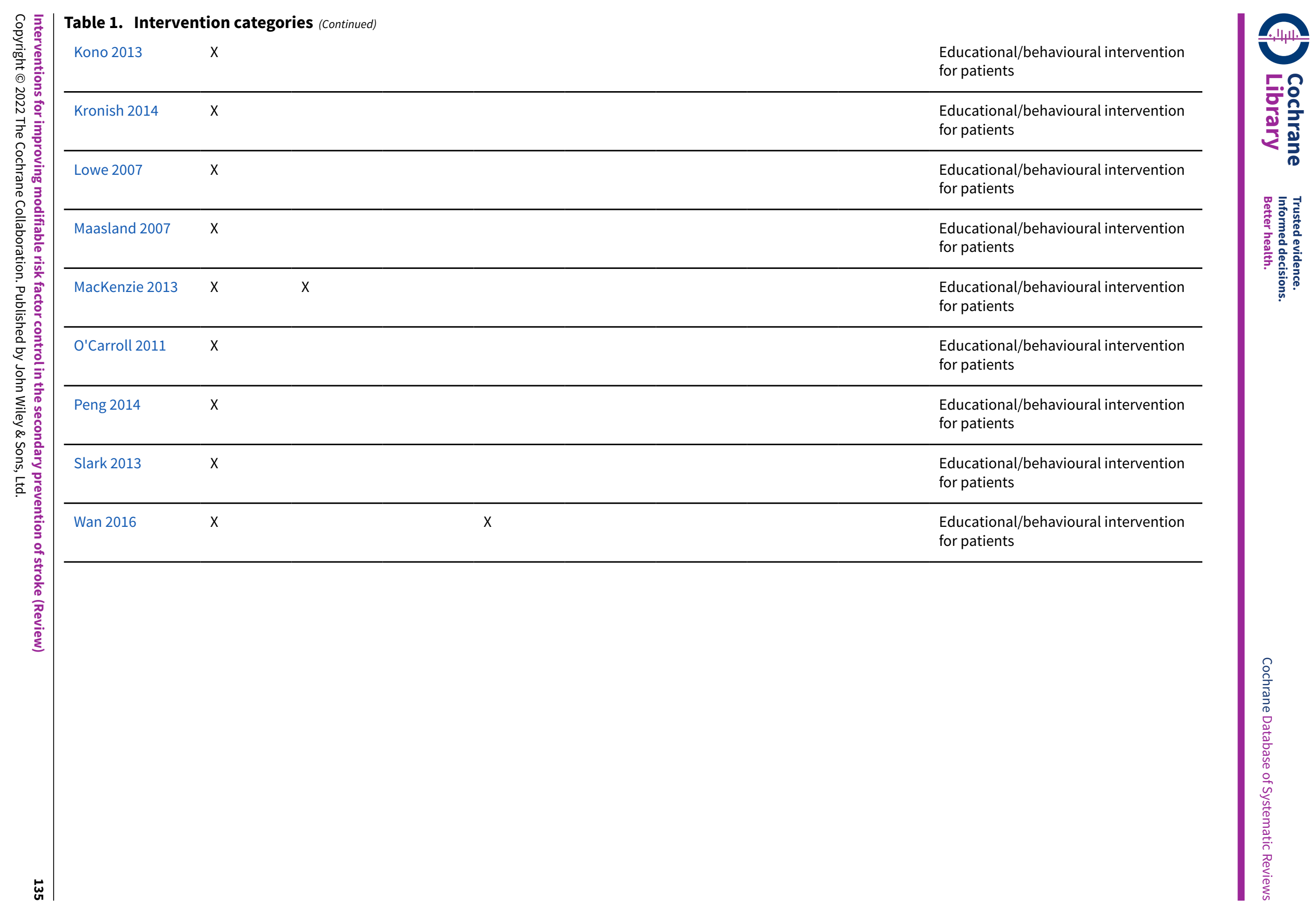




\section{APPENDICES}

\section{Appendix 1. CENTRAL search strategy}

1. MeSH descriptor: [Cerebrovascular Disorders] explode all trees

2. ((cva or stroke or poststroke or (post next stroke) or (transient next ischmic next attack) or TIA or ministroke or (mini next stroke)) near/6 (people or patient or outpatient or adult or survivor or victim or individual or client or population or community or subject)):ti,ab,kw (Word variations have been searched)

3. (cerebrovascular* or cerebral vascular):ti,ab,kw (Word variations have been searched)

4. (cerebral or cerebellar or brain* or vertebrobasilar):ti,ab,kw (Word variations have been searched)

5. (infarct* or isch $^{\star} \mathrm{mi}^{\star}$ or thrombo* or apoplexy or emboli^):ti,ab,kw (Word variations have been searched)

6. (4 and 5)

7. (cerebral or intracerebral or intracranial or brain* or cerebellar or subarachnoid):ti,ab,kw (Word variations have been searched)

8. (accident ${ }^{\star}$ or $\mathrm{h}^{\star}$ morrhag*):ti,ab,kw (Word variations have been searched)

9. (7 and 8)

10. $(1$ or 2 or 3 or 6 or 9$)$

11. MeSH descriptor: [Child] this term only

12. MeSH descriptor: [Infant] explode all trees

13. MeSH descriptor: [Pediatrics] explode all trees

14. (child* or neonat ${ }^{\star}$ or p? ediatric ${ }^{\star}$ or infant $\left.{ }^{\star}\right): t i, a b, k w$ (Word variations have been searched)

15. (11 or 12 or 13 or 14$)$

16. MeSH descriptor: [Patient Care Management] this term only

17. MeSH descriptor: [Comprehensive Health Care] this term only

18. MeSH descriptor: [Nursing Process] this term only

19. MeSH descriptor: [Nursing Assessment] explode all trees

20. MeSH descriptor: [Patient Care Planning] this term only

21. MeSH descriptor: [Case Management] this term only

22. MeSH descriptor: [Delivery of Health Care] this term only

23. MeSH descriptor: [Delivery of Health Care, Integrated] this term only

24. MeSH descriptor: [Managed Care Programs] 1 tree(s) exploded

25. MeSH descriptor: [Disease Management] this term only

26. MeSH descriptor: [Patient Care Team] explode all trees

27. MeSH descriptor: [Primary Health Care] this term only

28. MeSH descriptor: [Reminder Systems] this term only

29. MeSH descriptor: [Guideline Adherence] this term only

30. MeSH descriptor: [Home Care Services] this term only

31. MeSH descriptor: [Home Nursing] this term only 
32. MeSH descriptor: [Nursing Services] explode all trees

33. MeSH descriptor: [Professional Role] this term only

34. MeSH descriptor: [Community Health Services] this term only

35. MeSH descriptor: [Medical Records] this term only

36. MeSH descriptor: [Medical Records Systems, Computerized] this term only

37. MeSH descriptor: [Patient Education as Topic] this term only

38. MeSH descriptor: [Patient Compliance] 1 tree(s) exploded

39. MeSH descriptor: [Life Style] this term only

40. MeSH descriptor: [Health Promotion] this term only

41. MeSH descriptor: [Health Services Administration] this term only

42. MeSH descriptor: [Education, Medical, Continuing] this term only

43. MeSH descriptor: [Marketing of Health Services] this term only

44. MeSH descriptor: [Patient Participation] this term only

45. MeSH descriptor: [Quality of Health Care] this term only

46. MeSH descriptor: [Quality Assurance, Health Care] this term only

47. MeSH descriptor: [Exercise] this term only

48. MeSH descriptor: [Physical Fitness] this term only

49. MeSH descriptor: [Smoking Cessation] this term only

50. MeSH descriptor: [Diet] this term only

51. MeSH descriptor: [Diet, Fat-Restricted] this term only

52. MeSH descriptor: [Diet, Carbohydrate-Restricted] this term only

53. MeSH descriptor: [Diet, Reducing] this term only

54. MeSH descriptor: [Caloric Restriction] this term only

55. MeSH descriptor: [Alcohol Drinking] this term only and with qualifier(s): [Prevention \& control - PC]

56. MeSH descriptor: [Health Education] this term only

57. MeSH descriptor: [Community Health Planning] this term only

58. MeSH descriptor: [Communication] this term only

59. MeSH descriptor: [Communication Barriers] this term only

60. MeSH descriptor: [Information Dissemination] this term only

61. MeSH descriptor: [Interdisciplinary Communication] this term only

62. MeSH descriptor: [Nurse Clinicians] this term only

63. MeSH descriptor: [Nurse Practitioners] this term only

64. MeSH descriptor: [Risk Reduction Behavior] this term only

65. MeSH descriptor: [Pamphlets] this term only

66. MeSH descriptor: [Health Behavior] this term only 
67. MeSH descriptor: [Health Knowledge, Attitudes, Practice] this term only

68. MeSH descriptor: [Secondary Prevention] this term only

69. MeSH descriptor: [Preventive Health Services] this term only

70. (manag* near/3 care):ti,ab,kw (Word variations have been searched)

71. (management near/3 program):ti,ab,kw (Word variations have been searched)

72. (case near/3 manag*):ti,ab,kw (Word variations have been searched)

73. (patient near/3 management):ti,ab,kw (Word variations have been searched)

74. (home near/3 intervention):ti,ab,kw (Word variations have been searched)

75. (home next visit ${ }^{\star}$ ):ti,ab,kw (Word variations have been searched)

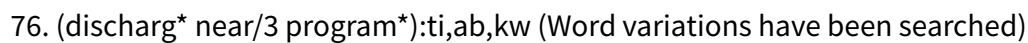

77. (practice next guideline $\left.{ }^{\star}\right): \mathrm{ti}, \mathrm{ab}, \mathrm{kw}$ (Word variations have been searched)

78. (discharg* near/3 plan*):ti,ab,kw (Word variations have been searched)

79. (comprehensive near/3 care):ti,ab,kw (Word variations have been searched)

80. (treatment near/3 plan*):ti,ab,kw (Word variations have been searched)

81. (nurse near/3 led):ti,ab,kw (Word variations have been searched)

82. (disease next management):ti,ab,kw (Word variations have been searched)

83. (multi next disciplin*):ti,ab,kw (Word variations have been searched)

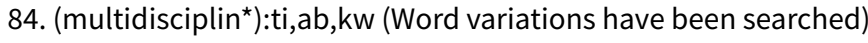

85. (secondary next prevention next clinic):ti,ab,kw (Word variations have been searched)

86. (reminder):ti,ab,kw (Word variations have been searched)

87. $\left(\right.$ recall $\left.^{\star}\right): t i, a b, k w$ (Word variations have been searched)

88. (nurse near/3 clinic):ti,ab,kw (Word variations have been searched)

89. (secondary next prevention near/3 intervention):ti,ab,kw (Word variations have been searched)

90. (secondary next prevention near/3 program*):ti,ab,kw (Word variations have been searched)

91. MeSH descriptor: [Appointments and Schedules] this term only

92. (appointment):ti,ab,kw (Word variations have been searched)

93. (outreach next nurs $\left.{ }^{\star}\right): t i, a b, k w$ (Word variations have been searched)

94. (outreach next visit*):ti,ab,kw (Word variations have been searched)

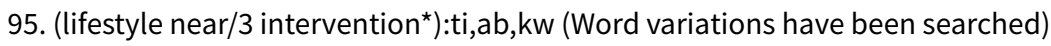

96. (physical next (activity or exercise)):ti,ab,kw (Word variations have been searched)

97. (aerobic):ti,ab,kw (Word variations have been searched)

98. (fitness):ti,ab,kw (Word variations have been searched)

99. (exercise near/3 (train* or intervention or program* or activity or regim)):ti,ab,kw (Word variations have been searched)

100. (nurs* next intervention $\left.{ }^{\star}\right): t i, a b, k w$ (Word variations have been searched)

101. (education* next program*):ti,ab,kw (Word variations have been searched) 
102. ((risk next factor $\left.{ }^{\star}\right)$ near/5 (modif* or reduc ${ }^{\star}$ or manage* or monitor ${ }^{\star}$ or self-manage $\left.\left.{ }^{\star}\right)\right):$ ti,ab,kw (Word variations have been searched)

103. $\{$ or $1-102\}$

104. (10 not 15)

105. (103 and 104)

\section{Appendix 2. MEDLINE (Ovid) search strategy}

1. exp Cerebrovascular Disorders/

2. ( (cva\$ or stroke\$ or poststroke\$ or post-stroke\$ or post stroke\$ or transient isch?emic attack\$ or TIA\$ or ministroke\$ or ministroke\$ or mini stroke\$) adj6 (people or patient $\$$ or inpatient $\$$ or outpatient $\$$ or adult $\$$ or survivor $\$$ or victim $\$$ or individual\$ or client $\$$ or population $\$$ or community or subject\$)).tw.

3. (cerebrovascular\$ or cerebral vascular).tw.

4. (cerebral or cerebellar or brain\$ or vertebrobasilar).tw.

5. (infarct\$ or isch?emi\$ or thrombo\$ or apoplexy or emboli\$).tw.

6.4 and 5

7. (cerebral or intracerebral or intracranial or brain\$ or cerebellar or subarachnoid).tw.

8. (accident\$ or h?emorrhag\$).tw.

9. 7 and 8

10. 1 or 2 or 3 or 6 or 9

11. exp Adolescent/

12. $\exp$ Child/

13. exp Infant/

14. exp Minors/

15. expPediatrics/

16. exp Puberty/

17. exp Schools/

18. (baby* or babies or infant ${ }^{\star}$ or infancy or neonat ${ }^{\star}$ or newborn* or postmatur ${ }^{\star}$ or prematur ${ }^{\star}$ or preterm*).tw.

19. (boy* or girl ${ }^{\star}$ or teen $\left.{ }^{\star}\right)$.tw.

20. (child* or kid or kids or preschool* or school age* or schoolchild* or toddler $\left.{ }^{\star}\right)$. tw.

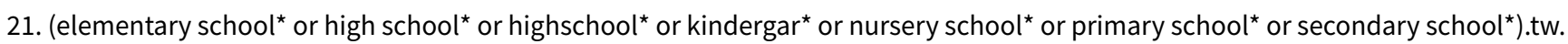

22. minors ${ }^{\star}$. tw.

23. (paediatric ${ }^{\star}$ or peadiatric ${ }^{\star}$ or pediatric $\left.{ }^{\star}\right)$.tw.

24. (prepubescen ${ }^{\star}$ or pubescen ${ }^{\star}$ or pubert $\left.{ }^{\star}\right)$.tw.

25. (youth or adolescen\$).tw.

26.11 or 12 or 13 or 14 or 15 or 16 or 17 or 18 or 19 or 20 or 21 or 22 or 23 or 24 or 25

27. 10 not 26

28. Patient Care Management/

29. Comprehensive Health Care/

Interventions for improving modifiable risk factor control in the secondary prevention of stroke (Review) 
30. Nursing Process/

31. exp Nursing Assessment/

32. Patient Care Planning/

33. Case Management/

34. delivery of health care/

35. Delivery of Health Care, Integrated/

36. exp Managed Care Programs/

37. Disease Management/

38. exp Patient Care Team/

39. exp Primary Health Care/

40. Reminder Systems/

41. Guideline Adherence/

42. Home Care Services/

43. Home Nursing/

44. exp Nursing Services/

45. exp Professional Role/

46. Community Health Services/

47. Medical Records/ or Medical Records Systems, Computerized/

48. Patient Education as Topic/

49. exp Patient Compliance/

50. Life Style/

51. Health Promotion/

52. Health Services Administration/

53. Education, Medical, Continuing/

54. Marketing of Health Services/

55. Patient Participation/

56. Quality of Health Care/

57. Quality Assurance, Health Care/

58. Exercise/ or Physical Fitness/

59. Smoking Cessation/

60. Diet/ or Diet, Fat-Restricted/ or Diet, Carbohydrate-Restricted/ or Diet, Reducing/ or Caloric Restriction/

61. Alcohol Drinking/pc [Prevention \& Control]

62. Health Education/

63. Community Health Planning/

64. Communication/ or Communication Barriers/ or Information Dissemination/ or Interdisciplinary Communication/

Interventions for improving modifiable risk factor control in the secondary prevention of stroke (Review) 
65. Nurse Clinicians/

66. Nurse Practitioners/

67. Risk Reduction Behavior/

68. Pamphlets/

69. Health Behavior/

70. Health Knowledge, Attitudes, Practice/

71. Secondary Prevention/

72. Preventive Health Services/

73. (manag\$ adj3 care).tw.

74. (management adj3 program\$).tw.

75. (case adj3 manag\$).tw.

76. (patient adj3 management).tw.

77. (home adj3 intervention\$).tw.

78. (home adj visit\$).tw.

79. (discharg\$ adj3 program\$).tw.

80. (practice adj guideline\$).tw.

81. (discharg\$ adj3 plan\$).tw.

82. (comprehensive adj3 care).tw.

83. (treatment adj3 plan\$).tw.

84. (nurse\$ adj3 led).tw.

85. (diseaseadj management).tw.

86. multi-disciplin\$.tw.

87. multidisciplin\$.tw.

88. secondary prevention clinic\$.tw.

89. reminder\$.tw.

90. recall\$.tw.

91. (nurse adj3 clinic\$).tw.

92. (secondary prevention adj3 intervention\$).tw.

93. (secondary prevention adj3 program\$).tw.

94. "Appointments and Schedules"/

95. appointment\$.tw.

96. (outreach adjnurs\$).tw.

97. (outreach adj visit\$়).tw.

98. (lifestyle adj3 intervention\$).tw.

99. (nurs\$ adj intervention\$).tw.

Interventions for improving modifiable risk factor control in the secondary prevention of stroke (Review) 
100. (education\$ adj program\$).tw.

101. (physical adj (activit\$ or exercise\$)).tw.

102. (exercise adj3 (train\$ or intervention\$ or program\$ or activit\$ or regim\$)).tw.

103. aerobic.tw.

104. fitness.tw.

105. (risk factor\$ adj5 (modif\$ or reduc\$ or manage\$ or monitor\$ or self-manage\$)).tw.

106. 28 or 29 or 30 or 31 or 32 or 33 or 34 or 35 or 36 or 37 or 38 or 39 or 40 or 41 or 42 or 43 or 44 or 45 or 46 or 47 or 48 or 49 or 50 or 51 or 52 or 53 or 54 or 55 or 56 or 57 or 58 or 59 or 60 or 61 or 62 or 63 or 64 or 65 or 66 or 67 or 68 or 69 or 70 or 71 or 72 or 73 or 74 or 75 or 76 or 77 or 78 or 79 or 80 or 81 or 82 or 83 or 84 or 85 or 86 or 87 or 88 or 89 or 90 or 91 or 92 or 93 or 94 or 95 or 96 or 97 or 98 or 99 or 100 or 101 or 102 or 103 or 104 or 105

107. Randomized Controlled Trials as Topic/

108. Random Allocation/

109. Controlled Clinical Trials as Topic/

110. control groups/

111. clinical trials as topic/ or clinical trials, phase i as topic/ or clinical trials, phase ii as topic/ or clinical trials, phase iii as topic/ or clinical trials, phase iv as topic/

112. double-blind method/

113. single-blind method/

114. Placebos/

115. placebo effect/

116. Drug Evaluation/

117. Research Design/

118. randomized controlled trial.pt.

119. controlled clinical trial.pt.

120. (clinical trial or clinical trial phase i or clinical trial phase ii or clinical trial phase iii or clinical trial phase iv).pt.

121. (random\$ or RCT or RCTs).tw.

122. (controlled adj5 (trial\$ or stud\$)).tw.

123. (clinical\$ adj5 trial\$).tw.

124. ((control or treatment or experiment\$ or intervention) adj5 (group\$ or subject $\$$ or patient\$)).tw.

125. (quasi-random $\$$ or quasi random $\$$ or pseudo-random $\$$ or pseud or random $\$$ ).tw.

126. ((singl\$ or doubl\$ or tripl\$ or trebl\$) adj5 (blind\$ or mask\$)).tw.

127. placebo\$.tw.

128. controls.tw.

129. exp animals/ not humans.sh.

130.107 or 108 or 109 or 110 or 111 or 112 or 113 or 114 or 115 or 116 or 117 or 118 or 119 or 120 or 121 or 122 or 123 or 124 or 125 or 126 or 127 or 128

\section{130 not 129}


132. 27 and 106 and 131

\section{Appendix 3. Embase (Ovid) search strategy}

1. exp *cerebrovascular disease/

2. ( (cva\$ or stroke\$ or poststroke\$ or post-stroke\$ or post stroke\$ or transient isch?emic attack\$ or TIA\$ or ministroke\$ or ministroke\$ or mini stroke\$) adj6 (people or patient $\$$ or inpatient $\$$ or outpatient $\$$ or adult $\$$ or survivor $\$$ or victim $\$$ or individual\$ or client $\$$ or population \$ or community or subject\$)).tw.

3. (cerebrovascular\$ or cerebral vascular).tw.

4. (cerebral or cerebellar or brain\$ or vertebrobasilar).tw.

5. (infarct\$ or isch?emi\$ or thrombo\$ or apoplexy or emboli\$).tw.

6. 4 and 5

7. (cerebral or intracerebral or intracranial or brain\$ or cerebellar or subarachnoid).tw.

8. (accident\$ or h?emorrhag\$).tw.

9. 7 and 8

10. 1 or 2 or 3 or 6 or 9

11. exp adolescence/

12. exp adolescent/

13. exp child/

14. high school/

15. kindergarten/

16. middle school/

17. expnewborn/

18. nursery school/

19. exppediatrics/

20. primary school/

21. exp puberty/

22. school/

23. adoles ${ }^{\star}$.tw.

24. (baby ${ }^{\star}$ or babies or infant ${ }^{\star}$ or infancy or neonat ${ }^{\star}$ or newborn ${ }^{\star}$ or postmatur ${ }^{\star}$ or prematur ${ }^{\star}$ or preterm ${ }^{\star}$ ).tw.

25. (boy* or girl* or teen*).tw.

26. (child* or kid or kids or preschool ${ }^{\star}$ or school age ${ }^{\star}$ or schoolchild* or toddler $\left.^{\star}\right)$.tw.

27. (elementary school ${ }^{\star}$ or high school ${ }^{\star}$ or highschool* or kindergar $^{\star}$ or nursery school* or primary school ${ }^{\star}$ or secondary school ${ }^{\star}$ ).tw.

28. minors*.tw.

29. ( paediatric $^{\star}$ or peadiatric ${ }^{\star}$ or pediatric $\left.{ }^{\star}\right)$. .tw.

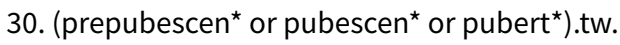

31. 11 or 12 or 13 or 14 or 15 or 16 or 17 or 18 or 19 or 20 or 21 or 22 or 23 or 24 or 25 or 26 or 27 or 28 or 29 or 30

32. $10 \operatorname{not} 31$

Interventions for improving modifiable risk factor control in the secondary prevention of stroke (Review) 
33. patient care planning/

34. case management/

35. health care delivery/

36. integrated health care system/

37. disease management/

38. reminder system/

39. *medical record/

40. health education/

41. patient education/

42. * patient compliance/

43. lifestyle modification/ or lifestyle/

44. health promotion/

45. medical education/

46. patient participation/

47. *exercise/ or aerobic exercise/ or fitness/ or * physical activity/

48. *smoking cessation/

49. *diet/ or low calory diet/ or low carbohydrate diet/ or low fat diet/ or diet restriction/

50. alcohol consumption/

51. health care planning/

52. interdisciplinary communication/

53. information dissemination/

54. risk reduction/

55. healthbehavior/

56. secondary prevention/

57. preventive medicine/

58. risk management/

59. medical specialist/

60. medical information/

61. (manag\$ adj3 care).tw.

62. (management adj3 program\$).tw.

63. (case adj3 manag\$).tw.

64. (patient adj3 management).tw.

65. (home adj3 intervention\$).tw.

66. (home adj visit\$).tw.

67. (discharg\$ adj3 program\$).tw. 
68. (practice adj guideline\$).tw

69. (discharg\$ adj3 plan\$).tw.

70. (comprehensive adj3 care).tw.

71. (treatment adj3 plan\$).tw.

72. (nurse\$ adj3 led).tw.

73. (diseaseadj management).tw.

74. (multi-disciplin\$ or multidisciplin\$).tw.

75. reminder\$.tw.

76. recall\$.tw.

77. (nurse adj3 clinic\$).tw.

78. (secondary prevention adj3 intervention\$).tw.

79. (secondary prevention adj3 program\$).tw.

80. appointment\$.tw.

81. (outreach adjnurs\$).tw.

82. (outreach adj visit\$).tw.

83. (lifestyle adj3 intervention\$).tw.

84. (nurs\$ adj intervention\$).tw.

85. (education\$ adj program\$).tw.

86. (physical adj (activit\$ or exercise\$)).tw.

87. (exercise adj3 (train\$ or intervention\$ or program\$ or activit\$ or regim\$)).tw.

88. aerobic.tw.

89. fitness.tw.

90. or/33-89

91. Randomized Controlled Trial/

92. Randomization/

93. Controlled Study/

94. control group/

95. clinical trial/ or phase 1 clinical trial/ or phase 2 clinical trial/ or phase 3 clinical trial/ or phase 4 clinical trial/ or controlled clinical trial/

96. Crossover Procedure/

97. Double Blind Procedure/

98. Single Blind Procedure/ or triple blind procedure/

99. latin square design/

100. Parallel Design/

101. Placebo/

102. Multicenter Study/

Interventions for improving modifiable risk factor control in the secondary prevention of stroke (Review) 
103. experimental design/ or experimental study/ or quasi experimental study/

104. experimental therapy/

105. drug comparison/ or drug dose comparison/

106. drug screening/

107. EVALUATION/ or "EVALUATION AND FOLLOW UP"/ or evaluation research/ or clinical evaluation/

108. METHODOLOGY/

109. "types of study"/

110. research subject/

111. Comparative Study/

112. "systematic review"/

113. Meta Analysis/

114. random\$.tw.

115. (controlled adj5 (trial\$ or stud\$)).tw.

116. (clinical\$ adj5 trial\$).tw.

117. ((control or treatment or experiment\$ or intervention) adj5 (group\$ or subject $\$$ or patient $\$)$ ).tw.

118. (surgical adj5 (group\$ or subject\$ or patient\$)).tw.

119. (quasi-random\$ or quasi random\$ or pseudo-random\$ or pseudo random\$).tw.

120. ((multicenter or multicentre or therapeutic) adj5 (trial\$ or stud\$)).tw.

121. ((control or experiment\$ or conservative) adj5 (treatment or therapy or procedure or manage\$)).tw.

122. ((singl\$ or doubl\$ or tripl\$ or trebl\$) adj5 (blind\$ or mask\$)).tw.

123. (coin adj5 (flip or flipped or toss\$)).tw.

124. latin square.tw.

125. versus.tw.

126. (cross-over or cross over or crossover).tw.

127. placebo\$.tw.

128. sham.tw.

129. (assign\$ or alternate or allocat\$ or counterbalance\$ or multiple baseline).tw.

130. controls.tw.

131. (treatment\$ adj6 order).tw.

132. (meta-analy\$ or metaanaly\$ or metaanaly\$ or systematic review or systematic overview).tw.

133. 91 or 92 or 93 or 94 or 95 or 96 or 97 or 98 or 99 or 100 or 101 or 102 or 103 or 104 or 105 or 106 or 107 or 108 or 109 or 110 or 111 or 112 or 113 or 114 or 115 or 116 or 117 or 118 or 119 or 120 or 121 or 122 or 123 or 124 or 125 or 126 or 127 or 128 or 129 or 130 or 131 or 132

134. Human/

135. Nonhuman/

136. 134 and 135 
137. 135 not 136

138. 133 not 137

\section{Appendix 4. CINAHL (EBSCO) search strategy}

1. (MH "Cerebral Ischemia+") OR (MH "Cerebral Hemorrhage") OR (MH "Stroke+") OR (MH "Intracranial Hemorrhage+")

2. (MH "Stroke Patients")

3. TX (cva* OR stroke* OR poststroke* OR post-stroke* OR "transient ischemic attack “ OR "transient ischaemic attack “ OR TIA OR ministroke* OR mini-stroke*)

4. TX (cerebrovascular* OR "cerebral vascular")

5. TX (cerebral OR cerebellar OR brain* OR vertebrobasilar)

6. TX (infarct ${ }^{\star}$ OR ischemi ${ }^{\star}$ OR ischaemi ${ }^{\star}$ OR thrombo* OR apoplexy OR emboli ${ }^{\star}$ )

7. S5 AND S6

8. TX (cerebral OR intracerebral OR intracranial OR brain* OR cerebellar OR subarachnoid)

9. TX (accident ${ }^{\star}$ OR hemorrhag* OR haemorrhag*)

10. S8 AND S9

11. S1 OR S2 OR S3 OR S4 OR S7 OR S10

12. (MH "Nursing Interventions")

13. (MH "Nursing Practice")

14. (MH "Advanced Nursing Practice")

15. (MH "Health Care Delivery")

16. (MH "Health Care Delivery, Integrated")

17. (MH "Disease Management")

18. (MH "Case Management")

19. (MH "Multidisciplinary Care Team")

20. (MH "Continuity of Patient Care+")

21. (MH "Patient Education")

22. (MH "Life Style Changes")

23. (MH "Behavior Modification")

24. (MH "Patient Compliance+")

25. (MH "Education, Medical, Continuing")

26. (MH "Education, Nursing, Continuing")

27. TX (manag* $n 3$ care)

28. TX (management $\mathrm{n} 3$ program ${ }^{\star}$ )

29. TX (case n3 manag*)

30. TX (patient $\mathrm{n} 3$ management)

31. TX (home N3 intervention*) 
32. TX "home visit*"

33. TX (discharg ${ }^{\star}$ n3 program ${ }^{\star}$ )

34. TX "practice guideline ${ }^{\star "}$

35. TX (discharg ${ }^{\star}$ n3 planning)

36. TX (comprehensive $\mathrm{n} 3$ care)

37. TX (treatment n3 plan*)

38. TX (nurse* n3 led)

39. TX "disease management"

40. TX multi-disciplin* OR TX multidisciplin*

41. TX "secondary prevention clinic*"

42. TX reminder ${ }^{\star}$ OR TX recall $^{\star}$

43. TX (nurse $\mathrm{n} 3$ clinic $^{\star}$ )

44. TX "secondary prevention" n3 (intervention* OR program*)

45. TX appointment*

46. TX "outreach nurs*"

47. TX "outreach visit*"

48. TX (lifestyle $\mathrm{n} 3$ intervention ${ }^{\star}$ )

49. TX "nurs* intervention*"

50. TX "education* program*"

51. TX ("physical activit" OR "physical exercise*")

52. TX exercise N3 (train* OR intervention* OR program* OR activit* OR regim)

53. TX fitness OR TX aerobic

54. TX "risk factor*" n3 (modif OR reduc* OR manage* OR monitor* OR self-manage*)

55. S12 OR S13 OR S14 OR S 15 OR S16 OR S17 OR S18 OR S19 OR S20 OR S21 OR S22 OR S23 OR S 24 OR S 25 OR S 26 OR S 27 OR S28 OR S29 OR S 30 OR S31 OR S32 OR S33 OR S34 OR S 35 OR S36 OR S37 OR S38 OR S39 OR S40 OR S41 OR S42 OR S43 OR S44 OR S45 OR S46 OR S47 OR S48 OR S49 OR S50 OR S51 OR S52 OR S53 OR S54 550,128

56. (MH "Random Assignment")

57. (MH "Random Sample+")

58. (MH "Crossover Design")

59. (MH "Clinical Trials+")

60. (MH "Comparative Studies")

61. (MH "Control (Research)+")

62. (MH "Control Group")

63. (MH "Factorial Design")

64. (MH "Quasi-Experimental Studies+")

65. (MH "Nonrandomized Trials")

Interventions for improving modifiable risk factor control in the secondary prevention of stroke (Review) 
66. (MH "Placebos")

67. (MH "Meta Analysis")

68. (MH "Clinical Nursing Research") OR (MH "Clinical Research+")

69. (MH "Community Trials")

70. (MH "Experimental Studies")

71. (MH "One-Shot Case Study") OR (MH "Pretest-Posttest Design+") OR (MH "Solomon Four-Group Design") OR (MH "Static Group Comparison") OR (MH "Study Design")

72. TI ("clinical trial" or "systematic review").

73. TX Random\$

74. TX ( ((singl\$ or doubl\$ or tripl\$ or trebl\$) adj25 (blind\$ or mask\$)) ) OR TX ( (cross?over or placebo \$ or control\$ or factorial or sham?) ) OR TX ( (clin\$ or intervention\$ or compar\$ or experiment\$ or preventive or therapeutic) adj10 trial\$) ) OR TX ( (counterbalance\$ or multiple baseline\$ or ABAB design\$) ) OR TX ( (meta?analys\$ or systematic review\$)

75. S56 OR S57 OR S58 OR S59 OR S60 OR S61 OR S62 OR S63 OR S64 OR S65 OR S66 OR S67 OR S68 OR S69 OR S70 OR S71 OR S72 OR S73 OR S74

\section{S11 AND S55 AND S75}

\section{Appendix 5. AMED (Ovid) search strategy}

1. CEREBRAL HEMORRHAGE/ or CEREBRAL INFARCTION/ or CEREBRAL ISCHEMIA/ or CEREBROVASCULAR ACCIDENT/ or STROKE/

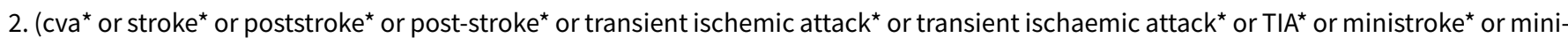
stroke $\left.{ }^{\star}\right) . t w$.

3. (people or patient ${ }^{\star}$ or outpatient ${ }^{\star}$ or inpatient ${ }^{\star}$ or adult ${ }^{\star}$ OR survivor ${ }^{\star}$ OR victim ${ }^{\star}$ or individual ${ }^{\star}$ or client ${ }^{\star}$ or population ${ }^{\star}$ or community or subject*).tw.

4. 2 and 3

5. (cerebrovascular* or cerebral vascular).tw.

6. (cerebral or cerebellar or brain* or vertebrobasilar).tw.

7. (infarct ${ }^{\star}$ or ischemi ${ }^{\star}$ or ischaemi ${ }^{\star}$ or thrombo* or apoplexy or emboli*).tw.

8. 6 and 7

9. (cerebral or intracerebral or intracranial or brain* or cerebellar or subarachnoid).tw.

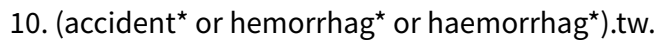

11.9 and 10

12. 1 or 4 or 5 or 8 or 11

13. DELIVERY OF HEALTH CARE/

14. PATIENT CARE MANAGEMENT/

15. PROGRAM EVALUATION/

16. PATIENT EDUCATION/

17. LIFE STYLE/

18. PREVENTION/

19. PATIENT COMPLIANCE/ 
20. PATIENT CARE TEAM/

\section{COMMUNITY HEALTH SERVICES/}

22. HEALTH PROMOTION/

\section{EXERCISE/}

\section{DIET/}

\section{SMOKING CESSATION/}

\section{HEALTH BEHAVIOR/}

27. (manag* adj3 care).tw.

28. (management adj3 program).tw.

29. (case adj3 manag $\left.{ }^{\star}\right)$.tw.

30. (patient adj3 management).tw.

31. (home adj3 intervention*).tw.

32. "home visit*".tw.

33. (discharg* adj3 program $\left.{ }^{\star}\right)$.tw.

34. "practice guideline*".tw.

35. (discharg* adj3 planning).tw.

36. (comprehensive adj3 care).tw.

37. (treatment adj3 plan*).tw.

38. (nurse ${ }^{\star}$ adj3 led).tw.

39. "disease management".tw.

40. multi-disciplin*.tw.

41. multidisciplin ${ }^{\star}$.tw.

42. "secondary prevention clinic".tw.

43. reminder ${ }^{\star}$.tw.

44. recall*.tw.

45. (nurse adj3 clinic*).tw.

46. ("secondary prevention" adj3 intervention*).tw.

47. ("secondary prevention" adj3 program).tw.

48. appointment ${ }^{\star} . t w$.

49. "outreach nurs"..tw.

50. "outreach visit*".tw.

51. (lifestyle adj3 intervention $\left.{ }^{\star}\right)$.tw.

52. "nurs ${ }^{\star}$ intervention ${ }^{\star}$.tw.

53. "education* program".tw.

54. ("physical activit" or "physical exercise*").tw. 
55. (exercise adj3 train*).tw.

56. (exercise adj3 intervention $\left.{ }^{\star}\right)$.tw.

57. (exercise adj3 program*).tw.

58. (exercise adj3 activit $\left.^{\star}\right)$.tw.

59. (exercise $\operatorname{adj} 3$ regim $\left.^{\star}\right)$.tw.

60. aerobic.tw.

61. fitness.tw.

62. ("risk factor*" adj5 modif*).tw.

63. ("risk factor*" adj5 reduc $\left.{ }^{\star}\right) . t w$.

64. ("risk factor ${ }^{\star} "$ adj5 manage $\left.{ }^{\star}\right) . t w$.

65. ("risk factor*" adj5 monitor $\left.{ }^{\star}\right)$.tw.

66. ("risk factor" adj5 self-manage*).tw.

67. or/12-66

68. RANDOMIZED CONTROLLED TRIALS/

69. CLINICAL TRIALS/

70. PLACEBOS/

71. DOUBLE BLIND METHOD/

72. random ${ }^{\star}$. tw.

73. placebo*.tw.

74.68 or 69 or 70 or 71 or 72 or 73

75. 12 and 67 and 74

\section{Appendix 6. BNI (Ovid) search strategy}

1. BNI STROKE/

2. BNI (cva* OR stroke* OR poststroke* OR post-stroke* OR "transient ischemic attack " OR "transient ischaemic attack " OR TIA* OR ministroke ${ }^{\star}$ OR mini-stroke $\left.{ }^{\star}\right) . t i, a b$

3. BNI (people OR patient ${ }^{\star}$ OR outpatient* OR inpatient ${ }^{\star}$ OR adult ${ }^{\star}$ OR survivor ${ }^{\star}$ OR victim ${ }^{\star}$ OR individual ${ }^{\star}$ OR client $^{\star}$ OR population* OR community OR subject*).ti,ab

4. BNI 2 AND 3

5. BNI (cerebrovascular* OR "cerebral vascular").ti,ab

6. BNI (cerebral OR cerebellar OR brain* OR vertebrobasilar).ti,ab

7. BNI (infarct* OR ischemi* OR ischaemi^ OR thrombo* OR apoplexy OR emboli*).ti,ab

8. BNI 6 AND 7

9. BNI (cerebral OR intracerebral OR intracranial OR brain* OR cerebellar OR subarachnoid).ti,ab

10. BNI (accident* OR hemorrhag* OR haemorrhag*).ti,ab

11. BNI 9 AND 10 
12. BNI 1 OR 4 OR 5 OR 8 OR 11

13. BNI PATIENTS: EDUCATION/

14. BNI NURSING: ROLE/

15. BNI CARE PLANS AND PLANNING/

16. BNI EVIDENCE BASED PRACTICE/

17. BNI MULTIDISCIPLINARY TEAMS/

18. BNI CONTINUITY OF CARE/

19. BNI PATIENTS: COMPLIANCE/

20. BNI (manag* ADJ3 care).ti,ab

21. BNI (management ADJ3 program*).ti,ab

22. BNI (case ADJ3 manag ${ }^{\star}$ ).ti,ab

23. BNI (patient ADJ3 management).ti,ab

24. BNI (home ADJ3 intervention*).ti,ab

25. BNI ”home visit^".ti,ab

26. BNI (discharg* ADJ3 program*).ti,ab

27. BNI "practice guideline*“.ti,ab

28. BNI (discharg* ADJ3 planning).ti,ab

29. BNI (comprehensive ADJ3 care).ti,ab

30. BNI (treatment ADJ3 plan*).ti,ab

31. BNI (nurse* ADJ3 led).ti,ab

32. BNI "disease management".ti,ab

33. BNI multi-disciplin*.ti,ab

34. BNI multidisciplin*.ti,ab

35. BNI "secondary prevention clinic ${ }^{\star}$ “.ti,ab

36. BNI reminder ${ }^{\star}$.ti,ab

37. BNI recall ${ }^{\star}$.ti,ab

38. BNI (nurse ADJ3 clinic ${ }^{\star}$ ).ti,ab

39. BNI ("secondary prevention" ADJ3 intervention^).ti,ab

40. BNI ("secondary prevention“ ADJ3 program).ti,ab

Interventions for improving modifiable risk factor control in the secondary prevention of stroke (Review) 130

Copyright @ 2014 The Cochrane Collaboration. Published by JohnWiley\& Sons, Ltd.

41. BNI appointment ${ }^{\star} \cdot \mathrm{ti}, \mathrm{ab}$

42. BNI "outreach nurs*".ti,ab

43. BNI "outreach visit*“.ti,ab

44. BNI (lifestyle ADJ3 intervention*).ti,ab

Interventions for improving modifiable risk factor control in the secondary prevention of stroke (Review) 
45. BNI "nurs* intervention*“.ti,ab

46. BNI "education* program*“.ti,ab

47. BNI ("physical activit*“ OR ”physical exercise*").ti,ab

48. BNI (exercise ADJ3 train*).ti,ab

49. BNI (exercise ADJ3 intervention*).ti,ab

50. BNI (exercise ADJ3 program ${ }^{\star}$ ).ti,ab

51. BNI (exercise ADJ3 activit*).ti,ab

52. BNI (exercise ADJ3 regim*).ti,ab

53. BNI aerobic.ti,ab

54. BNI fitness.ti,ab

55. BNI ("risk factor ““ ADJ5modif*).ti,ab

56. BNI ("risk factor*“ ADJ5reduc*).ti,ab

57. BNI ("risk factor*“ ADJ5 manage*).ti,ab

58. BNI ("risk factor*“ADJ5 monitor*).ti,ab

59. BNI ("risk factor*“ ADJ5 self-manage*).ti,ab

60. BNI 13 OR 14 OR 15 OR 16 OR 17 OR 18 OR 19 OR 20 OR 21 OR 22 OR 23 OR 24 OR 25 OR 26 OR 27 OR 28 OR 29

OR 30 OR 31 OR 32 OR 33 OR 34 OR 35 OR 36 OR 37 OR 38 OR 39 OR 40 OR 41 OR 42 OR 43 OR 44 OR 45 OR 46 OR

47 OR 48 OR 49 OR 50 OR 51 OR 52 OR 53 OR 54 OR 55 OR 56 OR 57 OR 58 OR 59

61. BNI random*.ti,ab

62. BNI placebo*.ti,ab

63. BNI trial.ti,ab

64. BNI 61 OR 62 OR 63

65. BNI 12 AND 60 AND 64

\section{Appendix 7. Web of Science Conference Proceedings Citation Index - Science search strategy}

Stroke* OR TIA OR "transient isch*mic attack" OR "cerebral infarct*“ OR "brain infarct*“ OR cerebrovascular IN TITLE AND

("secondary SAME prevention") OR ("recurrent stroke") OR (risk SAME reduc*) IN TOPIC

AND

intervention or program or service* or management IN TOPIC

AND

Proceedings paper IN DOCUMENT TYPE

\section{Appendix 8. Clinical Trials (www.clinicaltrials.gov) - search strategy}

1. Stroke*

2. TIA

3. "transient isch*mic attack" 
4. "cerebral infarct*"

5. "brain infarct*"

6. cerebrovascular

\section{Appendix 9. ISRCTN Registry (www.isrctn.com) - search strategy}

1. Stroke*

2. TIA

3. "transient isch*mic attack"

4. "cerebral infarct*"

5. "brain infarct*"

6. cerebrovascular

\section{Appendix 10. Stroke Trials Registry (www.strokecenter.org/trials/) - search strategy}

1. Stroke*

2. TIA

3. "transient isch*mic attack"

4. "cerebral infarct*"

5. "brain infarct ${ }^{\star "}$

6. cerebrovascular

7. disease management/

8. reminder system/

9. patient education/

10. lifestyle modification/ or lifestyle/

11. health promotion/

12. medical education/

13. patient participation/

14. health care planning/

15. secondary prevention/

16. preventive medicine/

Appendix 11. World Health Organization (WHO) International Clinical Trials Registry Platform (www.apps.who.int/ trialsearch/) - search strategy

1. Stroke*

2. TIA

3. "transient isch*mic attack"

4. "cerebral infarct*"

5. "brain infarct*"

6. cerebrovascular 
7. disease management/

8. reminder system/

9. patient education/

10. lifestyle modification/ or lifestyle/

11. health promotion/

12. medical education/

13. patient participation/

14. health care planning/

15. secondary prevention/

16. preventive medicine/

17. 1 OR 2 OR 3 OR 4 OR 5 OR 6 AND 7

18. 1 OR 2 OR 3 OR 4 OR 5 OR 6 AND 8

19. 1 OR 2 OR 3 OR 4 OR 5 OR 6 AND 9

20. 1 OR 2 OR 3 OR 4 OR 5 OR 6 AND 10

21. 1 OR 2 OR 3 OR 4 OR 5 OR 6 AND 11

22. 1 OR 2 OR 3 OR 4 OR 5 OR 6 AND 12

23. 1 OR 2 OR 3 OR 4 OR 5 OR 6 AND 13

24. 1 OR 2 OR 3 OR 4 OR 5 OR 6 AND 14

25. 1 OR 2 OR 3 OR 4 OR 5 OR 6 AND 15

261 OR 2 OR 3 OR 4 OR 5 OR 6 AND 16

WHAT'S NEW

\begin{tabular}{lll}
\hline Date & Event & Description \\
\hline 8 June 2022 & Amended & Change of corresponding author \\
\hline
\end{tabular}

\section{H I S T O R Y}

Protocol first published: Issue 6, 2011

Review first published: Issue 5, 2014

\begin{tabular}{lll}
\hline Date & Event & Description \\
\hline 3 April 2017 & $\begin{array}{l}\text { New citation required but conclusions } \\
\text { have not changed }\end{array}$ & The conclusions of the review remain unchanged. \\
\hline 3 April 2017 & New search has been performed & $\begin{array}{l}\text { This update included } 16 \text { new studies involving 25,819 addition- } \\
\text { al participants, resulting in a total of 42 studies including 33,840 } \\
\text { participants analysed in this review. The additional studies pro- } \\
\text { vided some evidence for the benefit of organisational interven- } \\
\text { tions achieving target levels for blood pressure. The update pro- }\end{array}$ \\
\hline
\end{tabular}




\begin{tabular}{lll}
\hline Date Event Description & Den
\end{tabular}

vided further evidence that educational and behavioural interventions were not associated with clear differences in any of the review outcomes.

\section{CONTRIBUTIONS OF AUTHORS}

Dr Bernadeta Bridgwood was principally responsible for data collection, analysis of data, interpretation of data and writing the update review in 2017 and 2018.

Dr Kate Lager contributed to the conception and design of the review. She was principally responsible for data collection, analysis of data, interpretation of data and writing the review.

Dr Amit K Mistri guided protocol development, and contributed to interpretation of the data and revising the review.

Professor Kamlesh Khunti guided protocol development, and contributed to interpretation of the data and revising the review.

Professor Andrew Wilson guided the conception and design of the review. He contributed to data collection, interpretation of data and revising the review.

Miss Priya Modi contributed to the interpretation of data and revising the review.

\section{DECLARATIONS OF INTEREST}

Dr Bernadeta Bridgwood acknowledges the support of the National Institute for Health Research Collaboration for the funding support of the Academic Clinical Fellowship in Primary Care.

Dr Kate Lager: none known.

Dr Amit Mistri has received speaker fees for talks on stroke from various companies manufacturing drugs for vascular disease including Boehringer-Ingelheim, Bayer, Bristol-Myers Squibb, Astellas Pharma, Pfizer and Astra Zeneca, and travel grants for conference attendance from Boehringer-Ingelheim. He has received a grant for an investigator-initiated study from Novo Nordisk.

Professor Kamlesh Khunti has acted as a consultant and speaker for Novartis, Novo Nordisk, Sanofi-Aventis, Lilly and Merck Sharp \& Dohme. He has received grants in support of investigator and investigator initiated trials from Novartis, Novo Nordisk, Sanofi-Aventis, Lilly, Pfizer, Boehringer Ingelheim and Merck Sharp \& Dohme. He has received funds for research, honoraria for speaking at meetings and has served on advisory boards for Lilly, Sanofi-Aventis, Merck Sharp \& Dohme and Novo Nordisk. Professor Khunti acknowledges the support of the National Institute for Health Research Collaboration for Leadership in Applied Health Research and Care - East Midlands (NIHR CLAHRC - EM), and the NIHR Leicester - Loughborough Diet, Lifestyle and Physical Activity Biomedical Research Unit.

Professor Andrew Wilson: none known.

Miss Priya Modi: none known

\section{SOURCES OF SUPPORT}

\section{Internal sources}

- Department of Health Sciences, Centre for Medicine, University of Leicester, UK

\section{External sources}

- No sources of support provided

\section{DIFFERENCES BETWEEN PROTOCOLANDREVIEW}

Haunton and Sett were not authors on the protocol but contributed to the first version of the full review. Modi and Bridgwood were not authors on the protocol nor the first version of the review but contributed to this updated version of the review

The title of the protocol was changed from Stroke services for risk reduction in the secondary prevention of stroke (Lager 2011 ) for the 2014 review (Lager 2014) following recommendations made by the Cochrane Stroke Group Editorial Team. 


\section{N D EX TERMS}

\section{Medical Subject Headings (MeSH)}

Behavior Therapy; Blood Pressure; Body Mass Index; Health Personnel [education]; Hypertension [prevention \& control]; Ischemic Attack, Transient [blood] [ ${ }^{*}$ prevention \& control]; Medication Adherence; Patient Education as Topic; Randomized Controlled Trials as Topic; Risk Factors; Secondary Prevention [*methods]; Stroke [blood] [ ${ }^{\star}$ prevention \& control]

\section{MeSH check words}

Aged; Humans; Middle Aged 